University of Louisville

ThinkIR: The University of Louisville's Institutional Repository

Electronic Theses and Dissertations

$8-2020$

\title{
A comprehensive study of the short-term variability of the migrating diurnal tide in the mesosphere and lower thermosphere.
}

Lokupatabendige Ashan Shivantha Vitharana

University of Louisville

Follow this and additional works at: https://ir.library.louisville.edu/etd

Part of the Atmospheric Sciences Commons

\section{Recommended Citation}

Vitharana, Lokupatabendige Ashan Shivantha, "A comprehensive study of the short-term variability of the migrating diurnal tide in the mesosphere and lower thermosphere." (2020). Electronic Theses and Dissertations. Paper 3516.

https://doi.org/10.18297/etd/3516

This Doctoral Dissertation is brought to you for free and open access by ThinkIR: The University of Louisville's Institutional Repository. It has been accepted for inclusion in Electronic Theses and Dissertations by an authorized administrator of ThinkIR: The University of Louisville's Institutional Repository. This title appears here courtesy of the author, who has retained all other copyrights. For more information, please contact thinkir@louisville.edu. 


\title{
A COMPREHENSIVE STUDY OF THE SHORT-TERM VARIABILITY OF THE MIGRATING DIURNAL TIDE IN THE MESOSPHERE AND LOWER THERMOSPHERE
}

\author{
By \\ Lokupatabendige Ashan Shivantha Vitharana \\ B.Sc., University of Colombo, Sri Lanka, 2012 \\ M.Sc., University of Louisville, Kentucky, USA, 2016 \\ A Dissertation \\ Submitted to the Faculty of the \\ College of Art and Science of the University of Louisville \\ in Partial Fulfillment of the Requirements \\ for the Degree of \\ Doctor of Philosophy \\ in Physics \\ Department of Physics and Astronomy \\ University of Louisville \\ Louisville, Kentucky
}

August 2020 
Copyright (C) 2020 by Lokupatabendige Ashan Shivantha Vitharana

All rights reserved 



\title{
A COMPREHENSIVE STUDY OF THE SHORT-TERM VARIABILITY OF THE MIGRATING DIURNAL TIDE IN THE MESOSPHERE AND LOWER THERMOSPHERE
}

\author{
By \\ Lokupatabendige Ashan Shivantha Vitharana \\ B.Sc., University of Colombo, Sri Lanka, 2012 \\ M.Sc., University of Louisville, Kentucky, USA, 2016
}

Dissertation approved on

July 23, 2020

by the following dissertation Committee:

\begin{tabular}{c}
\hline $\begin{array}{c}\text { Dissertation Director } \\
\text { Dr. Jian Du-Caines }\end{array}$ \\
\hline Dr. Jens Oberheide \\
\hline Dr. Xuwen Zhu \\
\hline Dr. Gamini Sumanasekera \\
\hline
\end{tabular}

Dr. Ming Yu 


\section{DEDICATION}

To my grandparents, for your unconditional guidance, support and love...

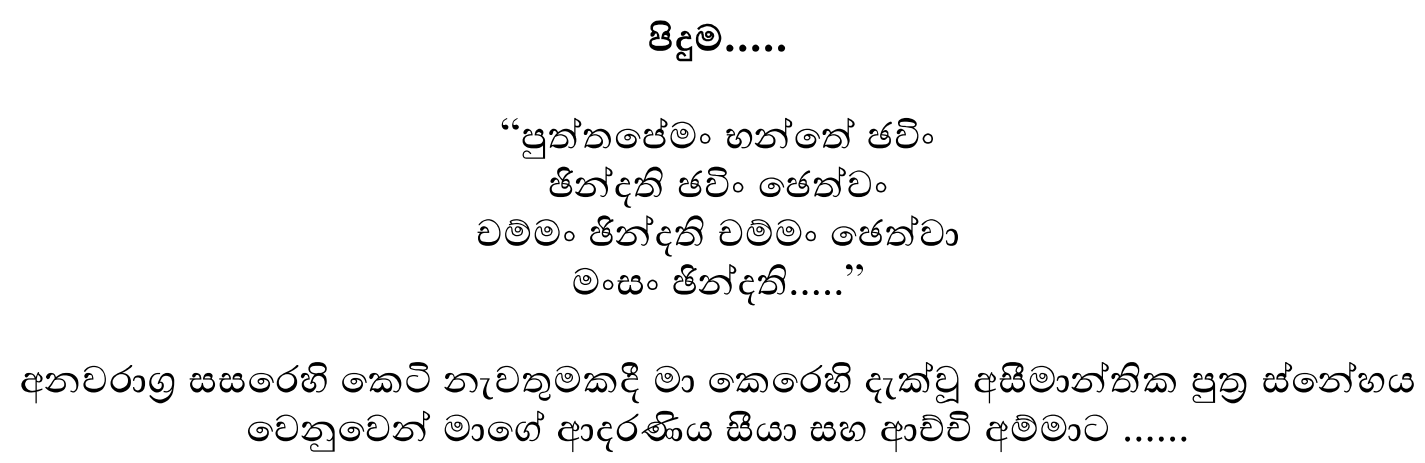

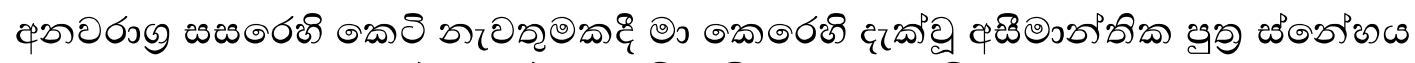

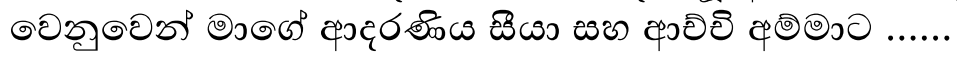




\section{ACKNOWLEDGMENTS}

The completion of this dissertation would not have been possible without the guidance and support of several individuals. First and foremost, I would like to express my sincere gratitude to my advisor Dr. Jian Du-Caines for her guidance, unconditional support, encouragement, and patience throughout the dissertation work. Thank you for spending countless hours reading and commenting on drafts of my work. Also, I would like to thank Dr. Du-Caines for giving me the great opportunity to work on outstanding space physics projects and to attend international and national conferences, which have greatly broadened my vision in this research field. Thank you for bringing out the best in me.

This work also would not have been possible without the support and assistance of Dr. Jens Oberheide from Clemson University, who provided the satellite data. I would like to thank Dr. Oberheide for giving me the opportunity and guidance. I would like to thank Dr. Xuwen Zhu from University of Alabama, for her guidance, valuable comments, and support over the past years. Also, I would like to thank Dr. William Ward from University of New Brunswick, Canada, for the valuable support to make this study succeed.

I would also like to thank the rest of my thesis committee, Dr. Gamini Sumanasekara and Dr. Ming Yu, for their insightful comments, advice, and for reviewing my dissertation. I must thank Dr. Chakram Jayanthi, Head of the Department of Physics and Astronomy and graduate student advisor Dr. Chris Davis for their support and guidance. 
I need to thank past members in our lab, Dr. Hongping Gu, Dr. Quan Gan, Austin Gornet, and David Warder for their support. Also, I need to thank staff members, Ms. Mary Gayle and Ms. Missy Klotz for their great help in the past six years and for all the paper works.

My deepest gratitude also goes to Dr. Gamini Sumanasekara and his wife Dr. Wasana Sumanasekara, families of Kasun, Saliya, Ruchira, Dilan, Manthila, Akila, Rajeeva and rest of the members of Sri Lankan student association, for treating me as one of their family members and helping me in countless ways to make this succeed. Thank you Ruchira, Saliya, Udika, Ruchini, and Roshanthi for always listening to me, supporting me, and encouraging me.

The financial support for this work through the grants from National Science Foundation (NSF) and NASA is gratefully acknowledged.

Finally, I am grateful to my family, my deceased grandfather, my loving grandmother, mother, father, my sister, and cousin brothers, Rukshan and Viraj for everything you have given me throughout my life. 


\begin{abstract}
A COMPREHENSIVE STUDY OF THE SHORT-TERM VARIABILITY OF THE MIGRATING DIURNAL TIDE IN THE MESOSPHERE AND LOWER THERMOSPHERE
\end{abstract}

\author{
Lokupatabendige Ashan Shivantha Vitharana
}

July 23,2020

Our ability to understand and predict space weather has become vital due to its significant societal impacts on communication, transportation, and national defense. One of the most exciting discoveries in the last decade has been the realization that tropospheric weather can strongly influence space weather. It is now recognized that the atmospheric waves (gravity waves, atmospheric tides, and planetary waves) play a key role in coupling the lower and upper atmosphere. The focus of this dissertation is to study atmospheric tides. While the climatology of tides has been extensively studied, little is known about the tidal weather (tidal variability $<30$-days). This dissertation constitutes a study to make the step from "seasonal/climatological tides" to "tidal weather/short-term variability" using the data from the extended Canadian Middle Atmosphere Model (eCMAM), and temperature observations from Sounding of the Atmosphere using Broadband Emission Radiometry (SABER) onboard the TIMED satellite. Particular attention is given to the short-term variability of the migrating diurnal tide (DW1). A hierarchy of statistical models, e.g., autoregressive (AR), vector AR (VAR), and parsimonious AR (PVAR), are developed to predict tidal weather based on the statistical properties such as the quasi 23-day oscillation and the spatial correlations found in the tidal weather. We can predict the next 
day's tidal weather at near $R^{2}=90 \%$ accuracy (correlation coefficient of 0.95 ). The total tidal variability is modulated on various temporal scales, hence a multi-linear regression model is fitted to the DW1 temperature amplitude using deterministic variables (solar cycle, ENSO, QBO, and the seasonal harmonics) and the fitted coefficients/amplitudes are examined. The absolute and relative variability of the shortterm tidal time series show significant 6 -month variation. Short-term tidal variability contributes $\sim 50-75 \%$ of the total tidal variability between 7 to 30-day window. Physical mechanisms for the short-term DW1 variability are also investigated using the eCMAM thermodynamic budget. Wave-mean flow interaction is mostly responsible for the tidal weather of DW1 in the mesosphere. Above $140 \mathrm{~km}$, short-wave solar heating becomes the governing force for the short-term DW1 variability. 


\section{TABLE OF CONTENTS}

Dedication ............................... iii

Acknowledgments ..................... iv

Abstract ............................... vi

List of Figures . . . . . . . . . . . . . . . . . xi

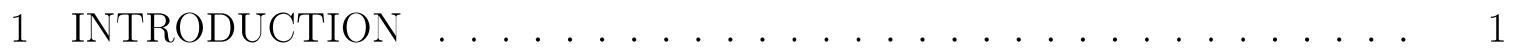

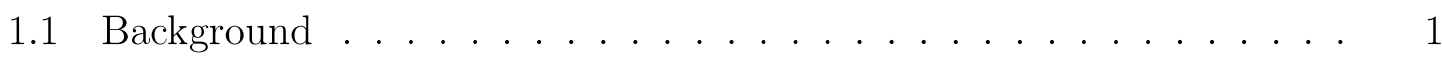

1.2 Motivation . . . . . . . . . . . . . . . . . . 7

1.3 Atmospheric Tides . . . . . . . . . . . . . . . . . . 15

1.3.1 Observations of Atmospheric Tides . . . . . . . . . . . 16

1.3.2 Modeling Effort on Atmospheric Tides . . . . . . . . . . . . 21

1.3.3 Spatial Variability in Tides. . . . . . . . . . . . . . . . 22

1.3.4 Temporal Variability in Tides . . . . . . . . . . . . . 28

1.4 Objectives . . . . . . . . . . . . . . . . . . . 36

1.5 Thesis Outline . . . . . . . . . . . . . . . . . . . 38

2 BASIC DYNAMICS OF THE ATMOSPHERE . . . . . . . . . . 40

2.1 Primitive Equations . . . . . . . . . . . . . . . . . . . 40

2.2 Zonal Mean and Eddies . . . . . . . . . . . . . . . . . . . . 43

2.3 Classical Tidal Theory . . . . . . . . . . . . . . . . . . . . . 44

2.3.1 Vertical Structure Equation: Forced and Free Solutions . . . . 48

2.3.2 Laplace's Tidal Equation . . . . . . . . . . . . . . . . . . . . . 49

2.4 Momentum and Thermodynamic Budget Equations . . . . . . . . . 51

3 THE ECMAM AND SABER DATA . . . . . . . . . . . 55

3.1 The eCMAM $\ldots \ldots \ldots \ldots \ldots \ldots \ldots \ldots \ldots \ldots$ 
3.2 SABER Short-term Tidal Diagnostics . . . . . . . . . . . . 68

3.3 Treatment of Data Gaps . . . . . . . . . . . . . . . . . . 72

4 STATISTICAL MODELING OF TIDAL WEATHER IN THE MESOSPHERE AND LOWER THERMOSPHERE . . . . . . . . . . . . . . 78

4.1 DW1 Tidal Climatology and Tidal Weather . . . . . . . . . . 79

4.2 Statistical Properties of Tidal Weather . . . . . . . . . . . . 80

4.3 Statistical Models of Tidal Weather . . . . . . . . . . . . . . 86

4.3.1 Model 1: Single Time Series Auto-Regression (AR) Model . . 86

4.3.2 Model 2: Vector Auto-Regressive (VAR) model . . . . . . . 87

4.3.3 Model 3: Parsimonious Vector Auto-Regressive (PVAR) Model 88

4.4 Obtain Model Coefficients . . . . . . . . . . . . . . . . 88

4.5 Tidal Weather Forecast . . . . . . . . . . . . . . . . . . 93

4.6 Comparison with SABER . . . . . . . . . . . . . . . . . . 97

4.7 Discussion and Conclusions . . . . . . . . . . . . . . . . . . . . . . 101

5 CHARACTERISTICS OF TOTAL DW1 VARIABILITY IN THE MESOSPHERE AND LOWER THERMOSPHERE . . . . . . . . . . . . . . 104

5.1 Total Tidal Variability of DW1 . . . . . . . . . . . . . 105

5.2 Multi-linear Regression Model . . . . . . . . . . . . . . . . . . 108

5.3 Climatological Mean of DW1 _ . . . . . . . . . . . . . . . 111

5.4 Interannual Variations of DW1 . . . . . . . . . . . . . . 112

5.5 Seasonal Variations of DW1 . . . . . . . . . . . . . . . . . 114

5.6 Long-term Variations in Short-term Tidal Variability . . . . . . . 116

5.7 Relative Importance of Temporal Variability . . . . . . . . . . . . 121

5.8 Total Tidal Forecast . . . . . . . . . . . . . . . . . . . . . . . 124

5.9 Discussion and Conclusions _. . . . . . . . . . . . . . . . . . . . 132

6 PHYSICAL MECHANISM STUDY OF THE SHORT-TERM TIDAL VARIABILITY IN THE MESOSPHERE AND THERMOSPHERE . . . . . . . 136

6.1 Introduction . . . . . . . . . . . . . . . . 136

6.2 Possible Mechanisms in the MLT region . . . . . . . . . . . . 140

6.2.1 Roles of Linear, Nonlinear Advection, and Solar Heating in the MLT Region . . . . . . . . . . . . . . . . . . . . . . 141

6.2.2 Role of Different Terms in the Linear Advection . . . . . . . . 149 
6.2.3 Non-local Effect from Solar Heating . . . . . . . . . . . . . . . 152

6.3 Physical Mechanisms in Thermosphere . . . . . . . . . . . . . . . 153

6.4 Conclusions . . . . . . . . . . . . . . . . . . . . . . 158

7 CONCLUSIONS AND FUTURE WORKS . . . . . . . . . . . . 161

7.1 Conclusions . . . . . . . . . . . . . . . . 163

7.1.1 Conclusions on Short-term Tidal Variability (Chapter 4) . . . 163

7.1.2 Conclusions on Total Tidal Variability (Chapter 5) . . . . . . 164

7.1.3 Conclusions on the Physical Mechanisms of Short-term Tidal Variability (Chapter 6) . . . . . . . . . . . . 166

7.2 Future Work . . . . . . . . . . . . . . . . . . 169

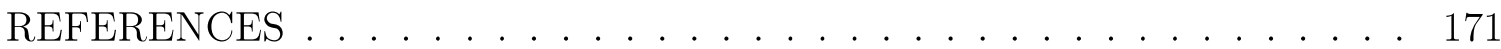

Appendix A: Acronyms . . . . . . . . . . . . . . 196

Appendix B: Comparision of DW1 T Between SABER Version 1.07 and 2.0 • 198

Appendix C: Correlations of Linear, Nonlinear Advection, Solar Heating, and Heating Rate . . . . . . . . . . . . . . . . . . . . . . 200

CURRICULUM VITAE . . . . . . . . . . . . . . . . . . . 202 


\section{LIST OF FIGURES}

1.1 Mean temperature profile at $3^{\circ} \mathrm{N}$ from the surface to $120 \mathrm{~km}$. Data are taken from the extended CMAM year 2004. . . . . . . . . 2

1.2 Ionosphere with the distribution of electron concentration. Credit: Dave Anderson and Tim Fuller-Rowell https://commons . wikimedia.org/wiki/ File:IonosphereProfileNOAA.png . . . . . . . . . . . . .

1.3 An overview of the physical processes that have been identified in the Earth's upper atmosphere. Credit: NASA's Scientific Visualization Studio - https://svs.gsfc.nasa.gov/4641 .............. 8

1.4 Climatological mean of the zonal wind amplitude $\left(\mathrm{m} \mathrm{s}^{-1}\right)$ of the diurnal tides: DW1, DE3, DW2 and DS0 as a function of latitude and height in

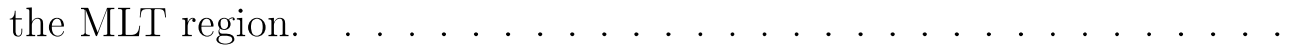

3.1 Normalized wavenumber spectra for diurnal tides $\mathrm{T}(\mathrm{K})$ at $100 \mathrm{~km}$ as a function of latitude from the eCMAM in March 2008. . . . . . . . . . 59

3.2 Climatology of zonal and seasonal mean background temperature (K) from the eCMAM30 as a function of latitude and altitude. . . . . . . . 61

3.3 Climatological mean of zonal wind $\left(\mathrm{m} \mathrm{s}^{-1}\right)$ from eCMAM30 as a function of latitude and altitude. Blue-colored (negative) regions are westward and the red-colored (positive) regions are eastward. . . . . . . . . . . .

3.4 Climatological mean of meridional wind $\left(\mathrm{m} \mathrm{s}^{-1}\right)$ from eCMAM30 as a function of latitude and altitude. Blue-colored (negative) regions are southward and the red-colored (positive) regions are northward. . . . . . . .

3.5 Climatological mean latitude-altitude structure of DW1 temperature amplitude (left) and phase (right) in the thermosphere (top panel), mesosphere (middle panel), and stratosphere (bottom panel) for March from the eCMAM30 (1979-2010) . . . . . . . . . . . . . . . . .

3.6 Seasonal variations of the DW1 T amplitude (K) at $165 \mathrm{~km}$ (top panel), $100 \mathrm{~km}$ (middle panel), and $45 \mathrm{~km}$ (bottom panel) from eCMAM30 as a function of latitude and month. . . . . . . . . . . . . .

3.7 SABER measurement track for 11 April 2008 as a function of latitude and altitude. . . . . . . . . . . . . . . . .

3.8 DW1 tidal amplitude as a function of altitude and day in 2007 from SABER. White space is missing data. . . . . . . . . . . . . 
3.9 Flow chart of filling data gaps in SABER with the Lomb-Scargle method. This figure is a derivative of Figure 1 from Hocke and Kämpfer (2009), used under a Creative Commons Attribution 4.0 International Public License: https ://creativecommons .org/licenses/by/4.0/. . . . . . . . .

3.10 Missing data percentage in SABER DW1 version 1.07 (top) between 20032012 and version 2.0 (bottom) between 2003-2017 as a function of altitude and latitude.

4.1 First panel: climatology mean amplitude $(\mathrm{K})$ of the DW1 T from January 2003 to June 2010 in the (left) eCMAM and (right) SABER. Second panel: long-term (periods $>30$ days) anomaly from the mean as a function of height and day in 2009 in eCMAM (left) and SABER (right). Third panel: Same as the second panel but for the short-term (periods $<30$ days) anomaly, for example, tidal weather in 2009. Fourth panel: Wavelet analysis of tidal weather at the equator and $100 \mathrm{~km}$ as a function of time

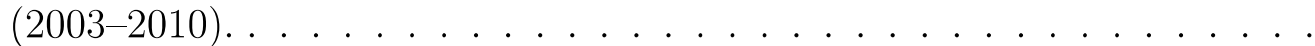

4.2 First panel: Auto-correlation of eCMAM DW1 tidal weather amplitude at $100 \mathrm{~km}$ and the equator. Second panel: Same as the first panel but for the SABER. Third panel: Auto-correlation of eCMAM DW1 tidal weather amplitude as a function of height and input data length. Fourth left panel: Average auto-correlation in eCMAM (blue line) and SABER (red line) as a function of the data input length. Fourth right panel: Histogram of auto-correlation lag for 6000-day input data length. . . . . . . . . . . .

4.3 Top panel: Spatial correlations between DW1 tidal weather at a particular height and DW1 tidal weather at all heights $(35-100 \mathrm{~km})$ at the Equator for the eCMAM (left) and SABER (right). Bottom panel: Same as top panel, but for spatial correlations between latitudes at $100 \mathrm{~km}$. . . . . . .

4.4 Prediction accuracy test of $\mathrm{AR}$, VAR and PVAR models using $R^{2}$ values as a function of different input data length used to calculate model coefficients. 90

4.5 Prediction accuracy of the AR model as a function of lag. . . . . . . . . . 91

4.6 ACC squared and R squared values as a function of height for 1-day prediction in the AR model. . . . . . . . . . . . . . . . . .

4.7 Prediction accuracy ( $R^{2}$ values) of fixed (solid) and variable (dashed) coefficients for different input data length (120-, 365-, and 2000-day) as a function of height in the AR model. . . . . . . . . . . . . . . .

4.8 Predicted tidal weather of 2009 at $100 \mathrm{~km}$ and the Equator using coefficients calculated from 2000-day data in the 2003 - 2008 period (red line) and from 2000-day data in the 1979 - 1984 period (black line) and the original tidal weather time series (blue line). . . . . . . . . . . . .

4.9 Top left: Tidal weather anomaly in 2009 from the eCMAM.Bottom left: Predicted tidal weather anomaly in 2009 with the AR model. Right: Prediction accuracy (average $R^{2}$ values over heights) as a function of future

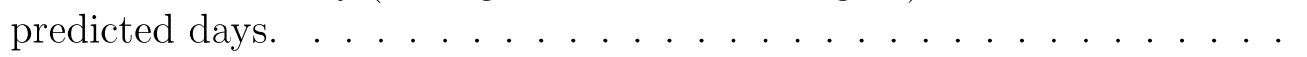

4.10 The 23 coefficients in the AR models from both eCMAM (blue lines) and SABER (orange lines) at 100 (top), 65 (middle), and $35 \mathrm{~km}$ (bottom). . . 
4.11 The 23 coefficients in the AR models for 3- day (first), 4- day (second), 6day (third), and 7- day averaging window in eCMAM at $100 \mathrm{~km}$. . . . .

4.12 Tidal weather anomaly in 2009 from SABER (top left), predicted tidal weather anomaly in 2009 from AR model with SABER coefficients (middle left), and predicted tidal weather anomaly in 2009 from AR model with eCMAM coefficients (bottom left). (Right) Prediction accuracy (average $R^{2}$ values over heights) as a function of future days for $\mathrm{AR}$ models with eCMAM (orange line) and SABER (blue line) coefficients. . . . . . . . . 100

5.1 The DW1 temperature amplitude $(\mathrm{K})$ as a function of latitude and time (from January 2003 to June 2010) at $95 \mathrm{~km}$ from the eCMAM (top) and SABER (bottom). . . . . . . . . . . . . . . . . . . . . . . . . . . . eCMAM (top) and SABER (bottom). The cross-hatched regions indicate the cone of influence (within the cone of influence, the results are not significant) and the solid black lines represent the 95\% confidence level. .

5.3 Normalized indices used in the multi-linear regression fitting: ENSO (top), QBO (middle), and solar flux (bottom). . . . . . . . . . . . .

5.4 Climatological mean of the DW1 T amplitude (K) in eCMAM (left) and SABER (right). The contour interval is $4 \mathrm{~K} . \ldots . . . . . . . . . .111$

5.5 Coefficients of the DW1 $\mathrm{T}(\mathrm{K})$ amplitude as a function of height and latitude to the deterministic interannual variability indices, e.g., solar cycle (top panel), ENSO (middle panel), and QBO (bottom panel), in the eCMAM (left) and SABER (right). Note that the contour intervals are different for eCMAM and SABER and for different indices. . . . . . . .

5.6 Amplitude of the DW1 T $(\mathrm{K})$ variability as a function of height and latitude due to the seasonal harmonics: $12,6,4$ and 3 months in the eCMAM (left) and SABER (right).

5.7 Monthly absolute variability of the short-term DW1 tidal amplitude in eCMAM at $100 \mathrm{~km}$ and the equator (first panel) and its Fourier spectrum (second panel). Similar as the first two panels, but for monthly relative variability (third panel) and the corresponding Fourier spectrum (fourth panel). The orange line is the $95 \%$ significance test with Monte Carlo of 1000 white noise time series. . . . . . . . . . . . . . . . .

5.8 Box plot of the absolute variability in March and June for eCMAM (top left), SABER (bottom left) and relative variability for eCMAM (top right) and SABER (bottom right) at $85 \mathrm{~km}$ equator. . . . . . . . . . .

5.9 DW1 total tidal variability (first panel), tidal variability in interannual (second panel), seasonal and intraseasonal (third panel), and short-term (fourth panel) time scales at $95 \mathrm{~km}$ in 2009. . . . . . . . . . . . . . 122

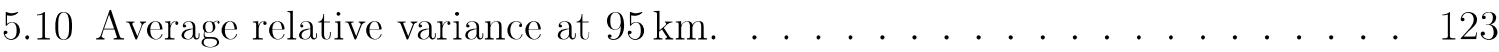


5.11 Actual long-term (top left), short-term (middle left), and total variability (bottom left) and fitted long-term (top right), predicted short-term (middle right), and predicted total variability (bottom right) of eCMAM DW1 $\mathrm{T}$ amplitude (in K) at the equator in 2009 as a function of height and day of the year. . . . . . . . . . . . . . . . . .

5.12 Actual long-term (top left), short-term (middle left), and total variability (bottom left) and fitted long-term (top right), predicted short-term (middle right), and predicted total variability (bottom right) of SABER DW1 $\mathrm{T}$ amplitude (in K) at the equator in 2014 as a function of height and day of the year. . . . . . . . . . . . . . . . . . .

5.13 Predicted accuracy of the long-term (top), short-term (middle), and total tide (bottom) at $95 \mathrm{~km}$ equator for eCMAM (blue line) and SABER (red

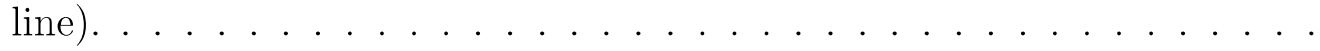

5.14 eCMAM DW1 tide in 1990 (first row), 2005 (second row), SABER 2011 (third row), and 2012 (fourth row) with respective Fourier spectrums. . .

6.1 Climatological mean of DW1 T time tendency (left top), linear advection (left middle), nonlinear advection (left bottom), solar heating (right top), and average heating rates at the equator (right bottom). . . . . . . .

6.2 Standard deviations of short-term DW1 T time tendency (top left), linear advection (top right), nonlinear advection (bottom left), and solar heating (bottom right).

Short-term correlation between DW1 T time tendency and linear advection (top panel), nonlinear advection (middle panel), and solar heating (bottom panel) in the mesosphere as a function of latitude and height from the eCMAM30. . . . . . . . . . . . . . . . . . . . . . .

6.4 Yearly short-term correlation between DW1 $\mathrm{T}$ time tendency and linear advection at the equator from the eCMAM30. . . . . . . . . .

6.5 DW1 time tendency (first panel), linear advection (second panel), nonlinear advection (third panel), and solar heating at $70 \mathrm{~km}$ in the equator. .

6.6 Climatological mean of time tendency (top left panel), linear advection term 1 (top right panel), term 2 (middle left panel), term 3 (middle right panel), term 4 (bottom left panel), and term 5 (bottom right panel) as a function of height and latitude. . . . . . . . . . . . . . . . . . .

6.7 Short-term correlation between DW1 T time tendency and linear advection term five as a function of latitude and height from the eCMAM30. .

6.8 Correlations between short-term variability of solar heating at all heights

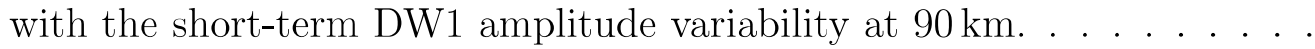

6.9 Climatological means of DW1 time tendency (top left panel), linear advection (top right panel), nonlinear advection (bottom left panel), and solar heating (bottom right panel). . . . . . . . . . . . . . .

6.10 Short-term correlation between DW1 T time tendency and linear advection (top panel), nonlinear advection (middle panel), and solar heating (bottom panel) in the thermosphere. 
6.11 DW1 time tendency (top) and solar heating (middle) at $165 \mathrm{~km}$ in the equator and their power spectrum (bottom). . . . . . . . . 157

B1 Coefficients of the DW1 T variability due to deterministic interannual variability indices, e.g., solar cycle (top panel), ENSO (middle panel), and QBO (bottom panel), in the SABER version 1.07 (left) and SABER version 2.0 (right) as a function of height and latitude. . . . . . . . . . . 198

B2 Amplitude of the DW1 T variability as a function of height and latitude due to the seasonal harmonics: 12,6 , and 3 months in the SABER version 1.07 (left) and SABER version 2.0 (right). . . . . . . . . . . . . . 199

C1 Correlation between DW1 T time tendency and linear advection (top panel), nonlinear advection (middle panel), and solar heating (bottom panel) in the mesosphere as a function of latitude and height from the eCMAM30. . . . . . . . . . . . . . . . . . . . 200

C2 Correlations between solar heating at all heights with the DW1 heating rate at $90 \mathrm{~km}$ as a function of latitude and height. . . . . . . . . . . . 201 


\section{CHAPTER 1}

\section{INTRODUCTION}

\subsection{Background}

The Earth, a tiny pale blue dot in the vast universe is the only place known to harbor life. Earth's atmosphere is a thin envelope of gases that surrounds the planet. The atmosphere keeps Earth naturally warm enough to sustain life on it through physical processes such as the greenhouse effect. It also prevents living organisms from extinction by filtering out the deadly cosmic rays, absorbing harmful ultraviolet radiation from the Sun, and burning external bodies (e.g., meteors) that collide with Earth. The composition of the atmosphere is largely made up of oxygen, nitrogen, a small number of other gases, and aerosols.

The atmosphere itself is not physically uniform but has significant variations in pressure and temperature with altitude. Although the pressure of the atmosphere decreases exponentially with altitude owing to tightly packed air molecules near the surface compared to higher altitudes, the temperature does not. Earth's atmosphere comprises four layers based on the vertical structure of the temperature gradient. The boundaries between layers are not clearly defined and can change with season and latitude. Figure 1.1 shows the mean placement of these layers: troposphere, stratosphere, mesosphere, and thermosphere that are separated by the tropopause, stratopause, and mesopause, and how the temperature changes with height from the ground up to space. 
The layer extending upward to about $10 \mathrm{~km}$ from the surface is known as the troposphere, where we live and most of the weather occurs. The temperature in this part of the atmosphere decreases with altitude at a rate of $\sim 6.5 \mathrm{~K} \mathrm{~km}^{-1}$ (lapse rate). The troposphere contains about $75 \%$ of the air mass and $99 \%$ of the water vapor. Changing the lapse rate from positive in the troposphere to negative in the stratosphere marks the upper boundary of the troposphere known as tropopause and it separates the troposphere and stratosphere.

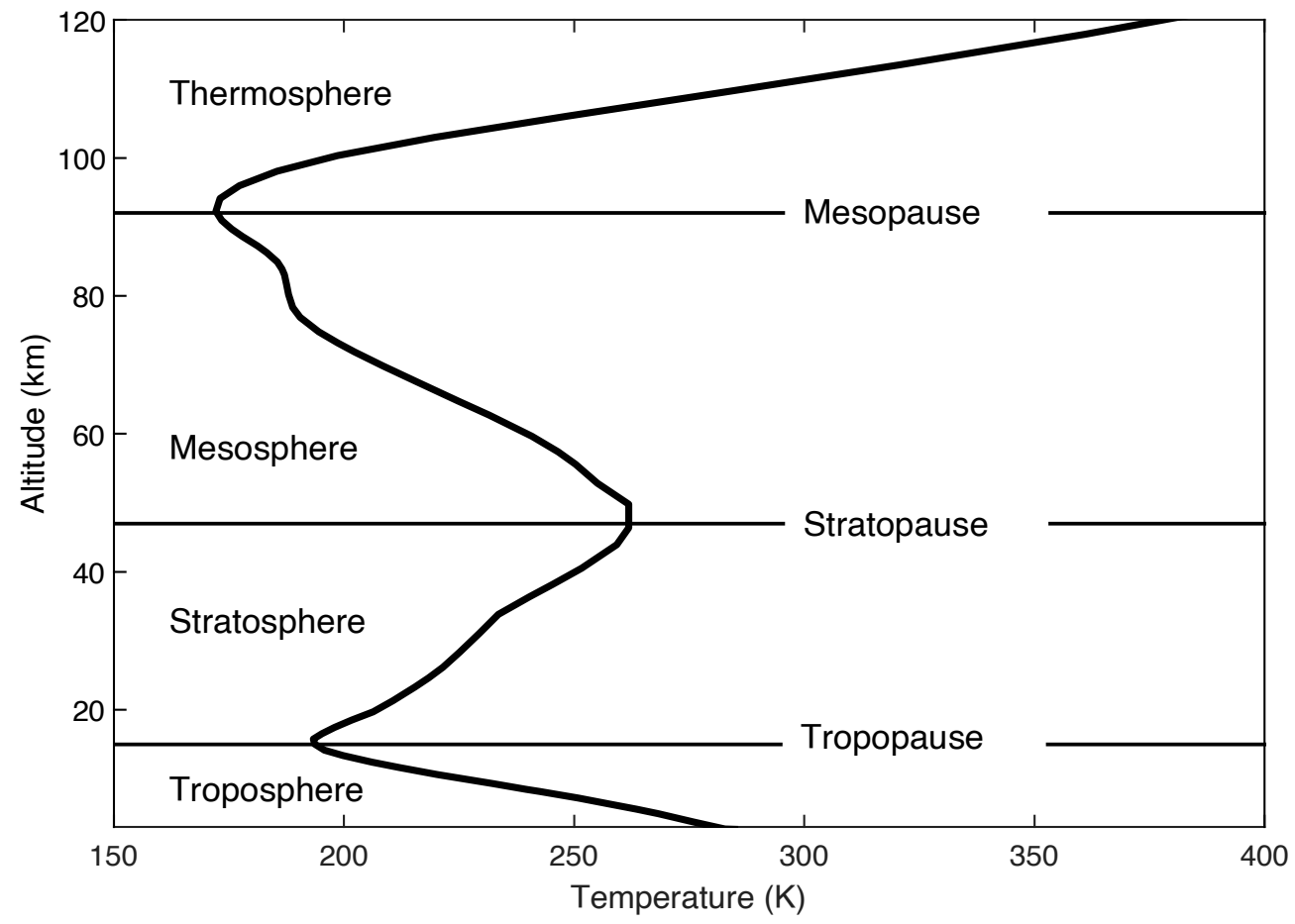

Figure 1.1. Mean temperature profile at $3^{\circ} \mathrm{N}$ from the surface to $120 \mathrm{~km}$. Data are taken from the extended CMAM year 2004.

The stratosphere extends from the tropopause up to $50 \mathrm{~km}$. The ozone layer, the important shield of gas that helps to preserve life on Earth from harmful solar rays, is found between 15 to $30 \mathrm{~km}$ of altitude within the stratosphere. Unlike the troposphere, the temperature in this region increases with altitude as a result of the absorption of ultraviolet (UV) radiation by ozone molecules. Unusual winter weather 
in the northern hemisphere may also link to the rapid increase of temperature (up to about $50{ }^{\circ} \mathrm{C}$ in just a couple of days) in the stratosphere known as the sudden stratospheric warming (SSW). Stratopause is the layer between the stratosphere and mesosphere which lies at about $50 \mathrm{~km}$.

The third layer of the Earth's atmosphere is the mesosphere. The mesosphere extends between stratopause to around $85-100 \mathrm{~km}$ and contains only one-thousandth of the atmosphere's mass. The physical and chemical processes differ from the stratosphere into the thermosphere, therefore the mesosphere is a very complex transition region in the atmosphere. Absorption of the solar radiation decreases due to diminishing ozone concentration and hence the temperature decreases with altitude. The highest clouds in the Earth's atmosphere known as "noctilucent clouds" or "polar mesospheric clouds" are located in the mesosphere and can be seen during astronomical twilight. The mesosphere has been nicknamed as the "ignorosphere" because it has always been hard for researchers to access since in situ measurements are difficult, hence less studied compared to the stratosphere and thermosphere. The mesosphere can only be accessed in-situ through rocketsondes, because its altitude is higher than the weather balloons range and is below the minimum altitude for orbital spacecraft. Scientists use instruments on sounding rockets to sample the mesosphere directly, but such flights are brief and infrequent. The mesosphere can be measured by remote sensing techniques such as radar, lidar, and satellite-borne passive sensors. The mesopause is defined as the temperature minimum that separates the mesosphere from the thermosphere and is the coldest region in Earth's atmosphere ( $170 \mathrm{~K})$. The mesopause altitude and temperature are also strongly modulated by the migrating diurnal tide at equinox and the semidiurnal tide at solstice (Xu et al., 2007; von Zahn et al., 1996). The middle atmosphere is known as the region which comprises the stratosphere and mesosphere. Conservation of energy allows atmospheric tides to grow to significant amplitudes in this region since the density decreases with altitude 
(Forbes and $\mathrm{Wu}, 2006$; Hagan and Forbes, 2003; Lindzen, 1966; Xu et al., 2007).

The thermosphere is the transition region from the atmosphere to space, and it extends from the mesopause up to the altitude between $500-1000 \mathrm{~km}$. However, the thermosphere does not have a well-defined upper limit, which changes with solar energy input, geomagnetic activities, local time, latitude, and season. Temperature in this region increases dramatically with altitude due to the absorption of X-ray and extreme ultraviolet radiation. The radiative energy is mainly absorbed by $\mathrm{O}$, $\mathrm{O}_{2}, \mathrm{~N}_{2}$ through ionization, heating, dissociation, and excitation. This region of the atmosphere is chemically active, and ionospheric processes become important.

In addition to the classification based on temperature, Earth's atmosphere has a number of different classification schemes. These are based on composition, ionization, and gravitation. In composition, the atmosphere is divided into the homosphere and heterosphere. The atmosphere is uniformly mixed up to about $100 \mathrm{~km}$ in the homosphere where eddy mixing dominates, and molecular diffusion dominates in the heterosphere where the composition is no longer $\sim 80 \% \mathrm{~N}_{2}$ and $20 \% \mathrm{O}_{2}$. The atmosphere consists of the neutral atmosphere, ionosphere, and plasmasphere in terms of ionization and can be divided into the barosphere and exosphere according to gravity.

The ionosphere is the region of the Earth's atmosphere with neutral atoms and molecules ionized by the high-energy X-rays and UV mainly from the Sun and cosmic radiation. The ionosphere lies between about 60 to $1000 \mathrm{~km}$ altitude from the surface and intermingles with parts of the mesosphere, thermosphere, and exosphere. As a result, this region is constantly affected by the conditions in the lower atmosphere and those in space (Fujiwara et al., 2009; Kamide and Chian, 2007; Roble, 2003). The discovery of the ionosphere goes back to early 1839, when Carl Friedrich Gauss postulated the existence of the ionosphere based on the observed variations of Earth's magnetic field.

Figure 1.2 shows the three main regions in the ionosphere known as the D layer, 
the $\mathrm{E}$ layer, and the $\mathrm{F}$ layer. The boundaries of these regions can vary during the day and from season to season. The D layer is the lowest region of the ionosphere starting at about $60-70 \mathrm{~km}$ and extending up to $90 \mathrm{~km}$. The D layer is mainly generated by ionizing the NO. Solar radiation at Lyman alpha and hard X-rays provides a significant source of ionization for the $\mathrm{D}$ region. Recombination rates are high in the D layer, hence absent of sunlight the number of ions drops, and the D layer disappears entirely during the night. Radio signals in the lower and medium frequency parts of the radio spectrum are attenuating in the D layer during daytime. The D layer reaches maximum ionization density at local noon.

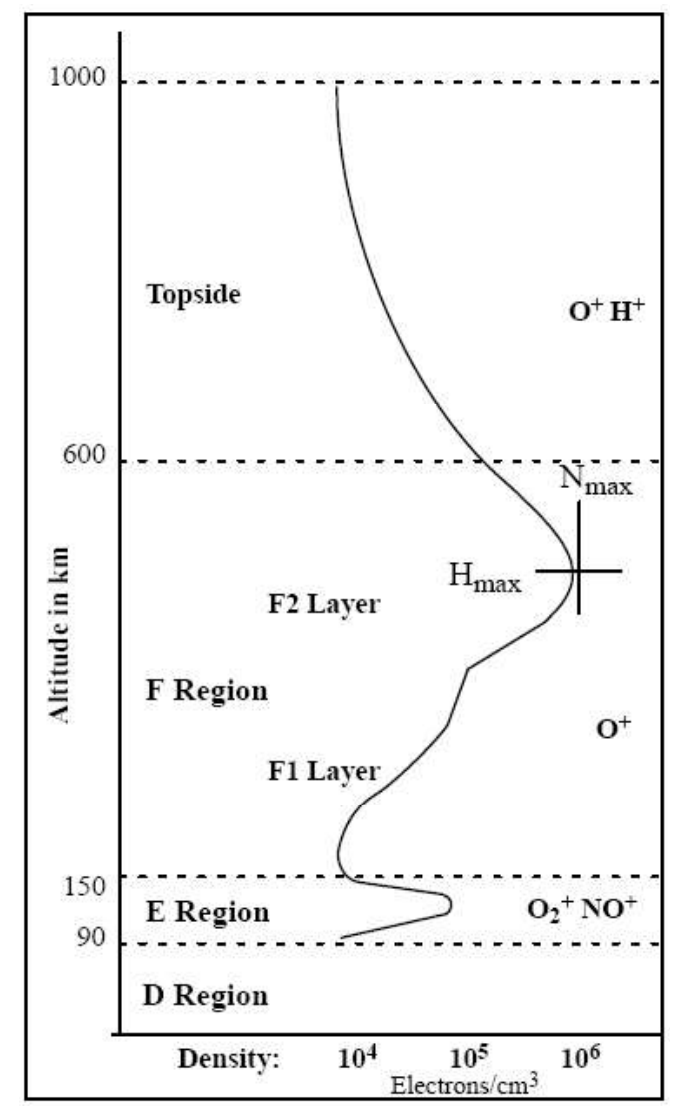

Figure 1.2. Ionosphere with the distribution of electron concentration. Credit: Dave Anderson and Tim Fuller-Rowell https://commons.wikimedia.org/wiki/File: IonosphereProfileNOAA.png

The E layer is above the D layer and exists between $90 \mathrm{~km}$ to about $150 \mathrm{~km}$. The E layer is also known as the Kennelly-Heaviside layer. Soft X-rays produce much of 
the ionization and the ionization density is about $10^{5}$ electrons per cubic centimeter during daytime. The most abundant ions in the $\mathrm{E}$ layer are $\mathrm{O}_{2}^{+}$and $\mathrm{NO}^{+}$. Intense clouds of ionization form in parts of the $\mathrm{E}$ layer known as sporadic $\mathrm{E}\left(\mathrm{E}_{\mathrm{s}}\right)$ layer which last for a few minutes to several hours. Though the causes of sporadic E layer are still not well understood, wind shear theory and meteor ionization mechanisms are the major factors in sporadic E layer formation (Yeh et al., 2014). The E layer becomes weakened during night because the number of ions plummets.

The outermost region in the ionosphere, the F region has the highest free electrons concentration in the atmosphere, on the order of $10^{6}$ free electrons per cubic centimeter. The $\mathrm{F}$ region splits into an upper $\mathrm{F}_{2}$ layer and lower $\mathrm{F}_{1}$ layer during the day and the $F_{1}$ layer merges into the $F_{2}$ layer after sunset. The $F_{1}$ layer exists between 150 to $220 \mathrm{~km}$ and the $\mathrm{F}_{2}$ layer exists between 250 to more than $500 \mathrm{~km}$ above the surface of Earth. The $\mathrm{F}_{2}$ layer can reflect high frequency (HF) radiowaves up to $35 \mathrm{MHz}$ during both day and night. Ionization in the $\mathrm{F}_{1}$ region is composed of $\mathrm{O}_{2}^{+}, \mathrm{O}^{+}$, and $\mathrm{N}_{2}^{+}$. The dominant positive ion in the $\mathrm{F}_{2}$ layer is $\mathrm{O}^{+}$.

The ionosphere layers can absorb, damp, and reflect radio waves. The radiowaves propegation in the ionosphere also affected by the geomagnetic activity. Geomagnetic activity is a measure of the state of the Earth's magnetic field. $K_{p}$ index is an indicator of the disturbances in the Earth's magnetic field. $\mathrm{K}_{\mathrm{p}}$ index has values that range between 0 and 9 . The values of the $K_{p}$ index gives a good indication of geomagnetic activity: values between 0 and 1 indicate quiet magnetic conditions and 9 representing a major storm that is likely to result in a blackout in HF ionospheric propagation for several hours. The pre-satellite era used the ionosphere to extend the range of transmissions by reflecting the radio waves back toward the Earth from the ionosphere. Much of the early work was carried out by radio-frequency engineers relevant to long range radio communication. In addition, present research has also focused on understanding the ionosphere as the home to the astronauts and Earth- 
orbiting satellites.

\subsection{Motivation}

Space weather is defined as the "conditions and changes on the Sun, in the solar wind, and within Earth's magnetosphere, ionosphere, and thermosphere that can influence the performance and reliability of space-borne and ground-based technological systems and endanger human life or health" (Eastwood, 2008). The large picture of this dissertation lies in the upper part of Earth's atmosphere, e.g., the IonosphereThermosphere-Mesosphere system. This region of the atmosphere is the transition layer from the Earth's atmosphere to space and is not only under the influence of the interplanetary space but also of lower atmospheric processes. Figure 1.3 presents an overview of the physical processes that have been identified in Earth's upper atmosphere. The space environment is filled with energetic particles, solar radiation and magnetic fields that drive "space weather" disturbances. Electric currents, photochemical processes of both neutrals and ionization, and plasma transport can occur rapidly in the ionosphere. At the same time, atmospheric waves carrying energy and momentum from terrestrial weather such as hurricanes, thermal tides and surface features can dramatically impact the circulation and variability of this region.

The need to understand and predict this space environment has been increasing in recent years due to its potentially significant societal impacts. The geomagnetically induced currents in power grid systems can cause widespreads effects including blackouts (for example, nine-hour outage of Hydro-Québec power grid on March 13, 1989). Variability in the ionosphere and plasmasphere can alter signals and affect the global communication and navigation systems. The accuracy of the radio signals used by the Global Positioning System (GPS) satellites to determine locations can be severely reduced by the ionospheric disturbances. Satellite radio communications can also be dampening or absorbed entirely by the ionospheric disruptions. Due to 


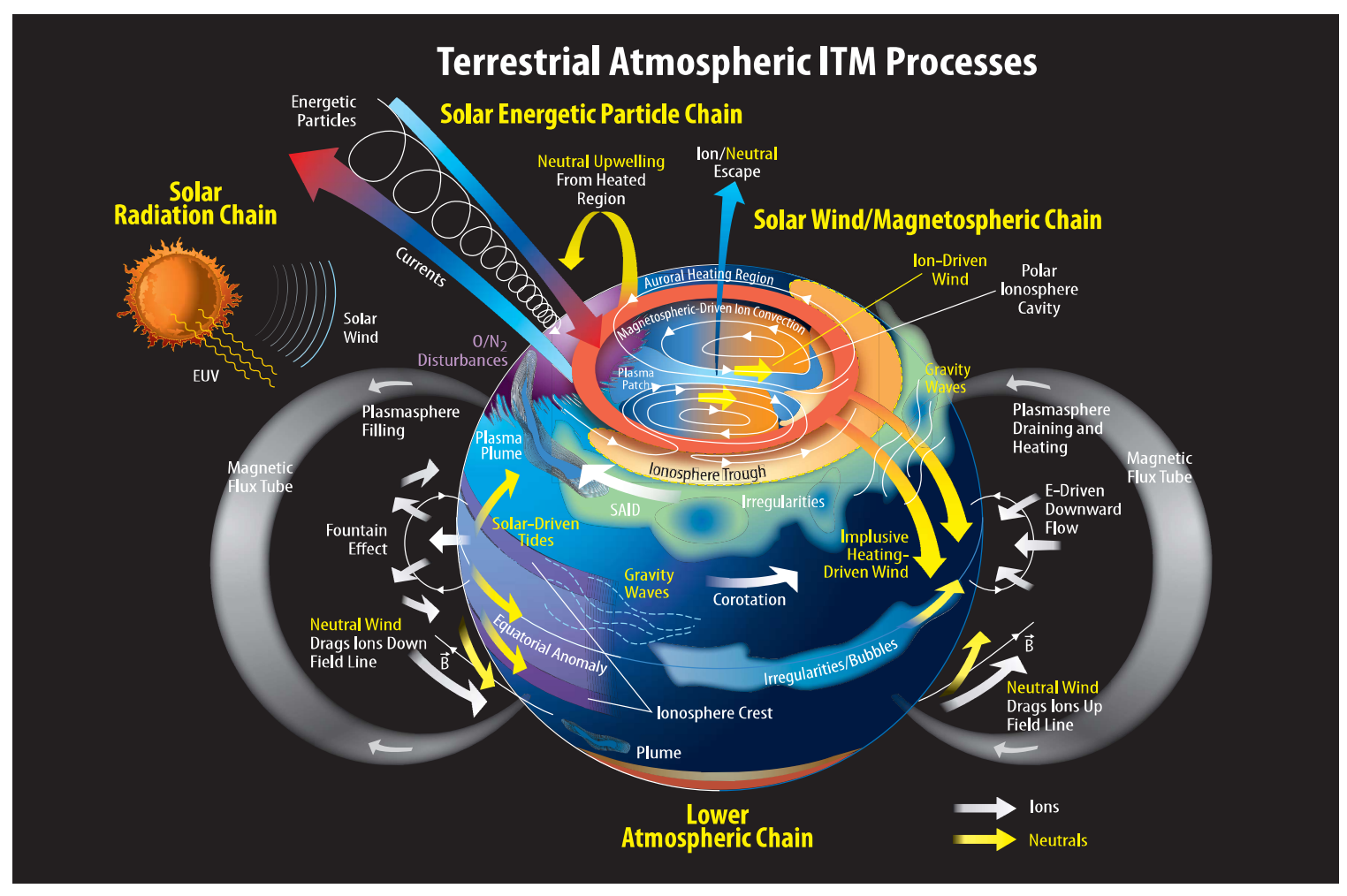

Figure 1.3. An overview of the physical processes that have been identified in the Earth's upper atmosphere. Credit: NASA's Scientific Visualization Studio https://svs.gsfc.nasa.gov/4641

the curvature of Earth, communication via geostationary satellites is not possible above the $\sim 80^{\circ}$ latitude. Thus, reliable HF radio communications are necessary for commercial airlines over cross-polar routes. In addition to the aviation industry, the maritime industry also uses HF communications in the Arctic region. To achieve long distance communication, HF radio relies on the reflections from the ionosphere. Space weather disturbances in the ionosphere can disrupt the HF communication in the polar region and can lead to unnecessary delays, maintenance, and increase operational costs. Remote sensing, safety of the International Space Station (ISS) and spacefarers, satellite malfunctions, and national security also can be adversely affected by conditions in the space environment. Space industry benefits and is connected with our everyday life in numerous ways, from simple mobile applications, to the matter of national defence. Space enterprise positions as the next trillion dollar industry. As 
published by the Space Foundation https://www. spacefoundation. org/ in quarter 2 of the Space Report 2019: the global space activity surpassed $\$ 414$ billion in 2018, with over 80 percent of total space economy is comercial (commercial infrastructure and support industries, and commercial space products and services) (Highfill et al., 2019). According to the U.S. Department of Commerce, the economic impact of space has grown to $\$ 5$ trillion annually (Ross, 2020). Important economic sectors like transportation and logistics, oil and gas, finance, agriculture, television, internet and wireless communication - all of these industries have been revolutionized by satellite services such as remote sensing, communications, positioning, navigation and timing. Starlink is the world largest low-Earth orbit satellite constellation being constructed by SpaceX to provide global broadband service including some of the most remote areas of the world. The starlink project will launch nearly 12,000 satellites into orbit by 2027 and will expand up to 42,000 satellites in the future. SpaceX, Blue Origin, and Virgin Galactic, all expect to offer suborbital travel to space tourists and high speed travel via outer space will revolutionize long distance travel. As the techniques further advance and our reliance on these techniques increases, the demands to be able to accurately predict space weather only rises. Predicting space weather is a complex problem due to the complicated interactions between many systems e.g., the Sun, the solar wind, the Earth's magnetic field, and the Earth's atmosphere.

It is known that activities in the Sun such as solar flares and coronal mass ejections (CME) have an enormous impact on the space weather of the upper atmosphere. One of the most exciting discoveries in space physics in recent years has been the realization that tropospheric weather can strongly affect the ionosphere and near Earth space during geomagnetically quiet times. Forbes et al. (2000) showed that under geomagnetic very quiet conditions $\left(\mathrm{K}_{\mathrm{p}}<1\right)$, the standard deviation of the ionospheric peak plasma density variability $\left(\mathrm{N}_{\mathrm{m}} \mathrm{F}_{2}\right)$ ranges from $25-30 \%$ of the mean at high frequencies (hours to 2 days period) and $15-20 \%$ at low frequencies $(2-30$ days 
periods) using the data from over 100 ionosonde stations and suggested that these variabilities are caused by lower atmospheric forcing. Rishbeth and Mendillo (2001) also found that the perturbations originating from the lower atmosphere are responsible for $\sim 15 \%$ of the $\mathrm{N}_{\mathrm{m}} \mathrm{F}_{2}$ standard deviation in medium solar activity. Observations during the deep solar minimum (2008 - 2009) and numerical models help for rapid progress in our understanding of how the lower atmosphere affects the upper atmosphere. These studies revealed that the variabilities (local-time, seasonal-latitudinal, day-to-day) in Earth's thermosphere and ionosphere (TI) during "quiet time" periods (geomagnetic quiet conditions and medium solar conditions) owe much to the perturbations originating from the lower atmosphere which transfer momentum and energy through propagation and generation of atmospheric waves. Mendillo et al. (2002) investigated ionosonde data with the Thermosphere-Ionosphere-Mesosphere and Electrodynamics General Circulation Model/Climate Community Model version 3 (TIME-GCM/CCM3) (Roble and Ridley, 1994) and found that the disturbances propagating upward from the lower atmosphere cause variations in $\mathrm{F}_{2}$-layer electron density of order 10-30\%. Simulations from the coupled Whole Atmosphere Model (WAM) (Akmaev et al., 2008) and Global Ionosphere-Plasmasphere Model (GIP) (Millward et al., 2001) revealed that under moderate solar activity and geomagnetic quiet conditions, perturbations from the lower atmosphere contribute to the $50 \%$ of the observed variability in the ionospheric peak $\mathrm{F}_{2}$ layer electron density $\left(\mathrm{N}_{\mathrm{m}} \mathrm{F}_{2}\right)$ (Fang et al., 2013). A major science goal of the Atmosphere-IonosphereMagnetosphere Interactions (AIMI) panel in the decadal strategy survey for Solar and Space Physics (2013 - 2022) is to "understand how tropospheric weather influences space weather" (National Research Council, 2013).

It is now recognized that the atmospheric waves play a key role in coupling the lower and upper atmosphere through modulation of thermospheric temperature and wind structure, influences on E region conductivities, generation of electric fields 
through dynamo action, and modification of thermospheric composition. The atmospheric waves responsible for transporting energy and momentum from the lower atmosphere to the upper atmosphere include gravity waves, atmospheric tides, and planetary waves. Variations in the propagation conditions and wave sources over time and space then cause upper atmosphere variability. Gravity waves (internal gravity or buoyancy waves) are restored by gravity and have relatively short periodicity (minutes to hours). They can be generated by short-lived terrestrial disturbances such as thunderstorms, hurricanes, and earthquakes. Atmospheric tides are persistent global oscillations which have periods that are integer fractions of the solar day and are mainly generated by the absorption of solar radiation in the troposphere and stratosphere, latent heat release from convective systems, and nonlinear wave-wave interactions. The restoring force that acts on atmospheric tides is also gravity, hence tides are a special class of gravity waves. Planetary waves are global oscillations with longer periods $(2 \sim 30$ days $)$ resulting from the conservation of potential vorticity and are influenced by the Coriolis force and pressure gradient. The restoring force for the planetary waves is the north-south gradient of background potential vorticity. Atmospheric tides grow exponentially with height and achieve large amplitudes in the upper atmosphere, and they may dissipate and deposit their energy and momentum therein. Some propagates all the way to the outermost region of the atmosphere and can cause significant redistribution in the ionospheric plasma. Gravity waves generated in the lower atmosphere can propagate up and deposit their momentum in the upper mesosphere and lower thermosphere. Planetary wave signature leaves its imprint in the upper atmosphere by modulation of upward propagating tides and gravity waves. In this dissertation, the focus of the study will be on one of these three types of atmospheric waves: atmospheric tides.

Atmospheric tides play an important role in the upper atmosphere variability. Temperature modulation caused by upward propagating tides is an important source 
for the midnight temperature maximum (MTM), an enhancement of the thermospheric temperature of $\sim 50$ to $200 \mathrm{~K}$ at low latitude in the near midnight (Fesen, 1996; Lei et al., 2011; Meriwether et al., 1986, 2008, 2011; Miyoshi et al., 2009). The WAM simulations suggest that the generation of MTM can trace to the tidal sources in the lower atmosphere primarily from the upward propagating migrating terdiurnal tide and other higher-order tides (Akmaev et al., 2009). The migrating terdiurnal tide also plays an important role in the seasonal variation of the MTM. MTMs of middle and high latitudes occur more often during the winter season in the northern hemisphere (Oliver et al., 2012; Ruan et al., 2014) and it might be associated with the hemispheric asymmetry of the migrating terdiurnal tide (Akmaev et al., 2010).

Modeling studies and the observations show a strong connection between tropospheric and ionospheric conditions (Hagan et al., 2007; Immel et al., 2006; Lühr et al., 2008). The wave number 4 pattern in the longitudinal structure of the F region equatorial ionization anomaly (EIA) was first observed by Sagawa et al. (2005) using nighttime airglow observations from the Far Ultraviolet Imager (FUV) on board the Imager for Magnetopause-to-Aurora Global Exploration (IMAGE) satellite. The EIA is an enhanced plasma density region found at $10^{\circ}$ to $20^{\circ}$ north and south of the magnetic equator. Later, Immel et al. (2006) proposed that the DE3 (Diurnal eastward-propagating tide with zonal wavenumber 3) was responsible for creating wave-4 variation in the observed airglow. Hagan et al. (2007) produced the observational results using the simulations from TIME-GCM and identified DE3 as responsible for the wave 4 structure in the EIA. The wave- 4 structure has been observed in various ionospheric parameters such as total electron content (Scherliess et al., 2008; Wan et al., 2008), ExB plasma drift (Hartman and Heelis, 2007; Kil et al., 2008), $\mathrm{O}^{+}$ airglow (England et al., 2006a), electron density profiles (Lin et al., 2007), daytime electric fields (England et al., 2006b). From a sun-synchronous satellite perspective, an oscillation with frequency $n \Omega$ and wave number $s$ appears as a zonal wave number 
$|s-n|$ structure with respect to longitude (see Equation 1.1 and 1.2). The DE3 $(n=1, s=-3)$ is seen as a wave-4 $(|s-n|=4)$ signature when observed from sunsynchronous satellite (Zhang et al., 2006). Apart from the DE3, a wave-4 structure can be forced by several nonmigrating tides: DW5, SW6, SE2, TW7, TE1 (the tidal nomenclature is explained in the Section 1.3) and stationary planetary wave number 4 (SPW4) since all are observed as wave-4 by quasi-sun-synchronous satellites (Oberheide et al., 2011). The equatorial electrojet (EEJ) is a narrow band of current that flows eastward in the daytime close to the magnetic equator. England et al. (2006b) showed that the DE3 modulates the wave-4 signature in EEJ around equinoxes. Lühr et al. (2008) found DE3 signature in the EEJ not only peaks during equinox seasons, but is also strong around the June solstice.

Recent studies clearly identified the ionospheric responses (perturbations of ion temperature, ion drift, and total electron density) during SSW events (Chau et al., 2012; Goncharenko et al., 2012; Goncharenko and Zhang, 2008; Liu et al., 2010b). Pedatella and Forbes (2010) studied the ionospheric response to the 2009 SSW using observations of the Global Positioning System total electron content (GPS-TEC) and revealed that the coupling between SSWs and ionosphere is related to the nonlinear interaction between the migrating semidiurnal tide SW2 and planetary waves. Enhancements in atmospheric lunar tides are also important for generating ionosphere variability during SSWs. Pedatella et al. (2012b) used WACCM simulations and found that the migrating semidiurnal lunar tide is enhanced $(\sim 60-70 \%)$ at mid to high latitudes during SSWs. Changes in the migrating semidiurnal lunar tide during SSWs can contribute up to an additional $\sim 30 \%$ of the total ionosphere variability. However, the overall influence of the lunar tide is depending on the phase of the moon relative to the timing of the SSW (Pedatella and Liu, 2013a). Lin et al. (2012) investigated the ionospheric tidal signatures during the 2009 SSW event using the ionospheric electron density observations from COSMIC (Constellation Observing 
System for Meteorology, Ionosphere and Climate) satellite and suggested that modifications of the migrating tidal components (DW1, SW2 and TW3) may be the major driver for producing ionospheric changes observed during SSW events. The physical processes driving the changes in the ionosphere during the January 2009 SSW were studied using WAM simulations and the study suggests that the change in magnitude and phase of the SW2 drives a stronger upward plasma drift (Fuller-Rowell et al., 2016).

Numerical studies have also found that tides can affect the thermospheric and ionosphere composition through affecting the transport in the mesosphere and lower thermosphere (MLT) by changing the vertical and meridional circulation. Numerical study from the Thermosphere-Ionosphere-Electrodynamics General Circulation Model (TIE-GCM) shows that the meridional circulation induced by dissipating DW1 and SW2 are responsible for the reduction of the atomic oxygen (Yamazaki and Richmond, 2013). The dissipation of the DW1 and SW2 at low latitudes causes a $30 \%$ increase in $\mathrm{O}_{2}$ density near the $\mathrm{F}_{2}$ layer leading to a $9 \%$ decrease in peak electron density $\left(\mathrm{N}_{\mathrm{m}} \mathrm{F}_{2}\right)$ (Jones et al., 2014). A detailed introduction on atmospheric tides will be provided in the following session.

A fundamental science question that remains is the short-term (1 day or less) variability in the IT system and its driving mechanisms. As described above, the lower atmospheric variability is also a major driver for the short-term variability of the IT system. This dissertation focuses on investigating the physical mechanisms and characteristics of the day to day tidal variability in the MLT region. Understand the governing physics behind short-term tidal variability is also important to space weather applications as it have consequences for a technological society. 


\subsection{Atmospheric Tides}

Atmospheric tides are global scale periodic oscillations in the Earth's atmosphere that can be observed in atmospheric fields, including temperature, density, wind, and pressure. Atmospheric tides are prominent features and play a major role in understanding the dynamics, energetics, and chemistry in the MLT region. Atmospheric tides can be classified into solar atmospheric tides and lunar atmospheric tides. Solar atmospheric tides are primarily driven by the zonally symmetric absorption of solar radiation from tropospheric water vapor and stratospheric ozone during the daily cycle of solar heating. Lunar atmospheric tides are induced by the Moon's gravitational pull. Apart from the absorption of solar radiation, solar atmospheric tides can be excited in several ways, e.g., latent heat release accompanying deep convective clouds in the troposphere, nonlinear wave-wave interactions between tides and planetary waves or gravity waves, and to a lesser extent the gravitational pull of the Sun (Chapman and Lindzen, 1970; Hagan and Roble, 2001; Lieberman et al., 2015; Lindzen, 1978; McLandress, 2002a,b; McLandress and Ward, 1994; Miyahara and Miyoshi, 1997; Siebert, 1961; Williams and Avery, 1996). In the middle and upper atmosphere, solar atmospheric tides dominate the tidal motion and are larger than the lunar atmospheric tides with the exception of SSW when Pekeris resonances might occur.

Solar heating can be approximated by a square wave profile so that Fourier transformation can be used to decompose the tidal components into harmonics of a solar day: diurnal $(24 \mathrm{~h})$, semidiurnal $(12 \mathrm{~h})$, and terdiurnal $(8 \mathrm{~h})$, etc. Mathematically, atmospheric tides can be represented by,

$$
A \cos (n \Omega t+s \lambda-\varphi)
$$

where $A$ is the tidal amplitude, $\varphi$ is the tidal phase, $\Omega$ is the rotation of the earth 
( $2 \pi /$ day $), \lambda$ is longitude, $t$ is universal time, $n(=1,2, \ldots)$ denotes a sub-harmonic of a solar day, and $s(=\ldots,-2,-1,0,1,2, \ldots)$ is the zonal wavenumber. Westward (Eastward) propagation corresponds to $s>0(s<0)$ and $n=1,2,3$ represent diurnal, semidiurnal, and terdirunal tides, respectively. Rewriting equation 1.1 in terms of local time $t_{L T}=t+\lambda / \Omega$, we have

$$
A \cos \left(n \Omega t_{L T}+(s-n) \lambda-\varphi\right)
$$

Solar tides can be separated into two components: migrating and nonmigrating. Migrating (sun-synchronous) tides propagate westward with the apparent motion of the Sun to a ground-based observer, $s=n$. Nonmigrating tides can propagate westward, eastward, or stationary with $s \neq n$. Solar tidal components are identified using the notation as follows: DWs or DEs denote a westward or eastward propagating diurnal tide, respectively, with absolute value of zonal wavenumber $s$. The sign of zonal wave number indicates westward or eastward propagation. For semidiurnal or terdiurnal tides, 'D' is replaced with ' $\mathrm{S}$ ' or ' $\mathrm{T}$ ' and D0 and S0 are standing diurnal and semidiurnal tides. For example, DW1 is migrating westward propagating diurnal tide of zonal wavenumber 1 , and DE3 is nonmigrating eastward propagating diurnal tide of zonal wavenumber 3 .

\subsubsection{Observations of Atmospheric Tides}

The MLT region is of great scientific interest since it is the transition region between the lower and upper atmosphere. Atmospheric tides propagate through this region and play a significant role, leading to energy and momentum transport from lower to higher altitudes. Ground-based instruments and space-borne instruments have been used to investigate the tidal dynamics in the MLT region. Ground based remote sensing systems such as Radio Detection and Ranging (radar) and Light Detection and Ranging (lidar) systems provide observations with high temporal resolution of 
the measurements. The meteor radar (MR) measures the Doppler shift of the radio reflections from meteor trails to estimate the winds in the $80-120 \mathrm{~km}$ over the MR station. The medium frequency radar (MF) derives mesospheric winds from $60-100 \mathrm{~km}$. The meteor radars can provide continuous wind measurements with $24 \mathrm{~h}$ local time coverage and MF radar is poor at making wind measurements at night.

The lidar system is the optical equivalent of radar and often referred to as laser radar. Lidar allows the measurements of temperature and wind covering the height range from the ground up to $120 \mathrm{~km}$. The basic lidar techniques are Rayleigh lidar, resonance-fluorescence lidar, and Doppler lidar. The Rayleigh lidar observes the atmospheric density around $30-80 \mathrm{~km}$. Atmospheric temperature profiles can be obtained using these atmospheric density profiles. The resonance-fluorescence lidar is used to measure temperature and wind in the mesopause region between about $80-110 \mathrm{~km}$ height. This region presents layers of metallic atoms and ions such as $\mathrm{Na}, \mathrm{K}, \mathrm{Ca}, \mathrm{Li}$, and Fe due to meteorite impacts. For example, Na lidar determines temperature and wind using the Doppler broadening and shift of the $\mathrm{Na} \mathrm{D}_{2}$ line. The doppler lidar system is also used to measure atmospheric wind fields. Some lidars can also make measurements during day and night. However, lidars are limited by weather conditions. Atmospheric tidal variations in the middle atmosphere have been studied extensively using radar and lidar measurements (Chang and Avery, 1997; Chang et al., 2012; Fong et al., 2014; Forbes et al., 1995; Fricke-Begemann and Höffner, 2005; Fritts et al., 2010; Huang et al., 2013, 2018; Kumar et al., 2008; Leblanc et al., 1999; Mitchell et al., 2002; She et al., 2004; Tsuda et al., 1988; Yu et al., 2013).

Past observations of the MLT region were limited to ground-based measurements. These ground-based observations are limited in spatial coverage and can only resolve the region above the site, hence cannot provide global coverage. The lack 
of spatial coverage by ground-based instruments led to the development of satellite techniques. Satellite instruments can observe the Earth from orbit and can provide the much needed global coverage for tidal diagnostics. The Upper Atmosphere Research Satellite (UARS) launched from the space shuttle Discovery on September 15, 1991 can carry out systematic, comprehensive study of the Earth's stratosphere and mesosphere (Reber, 1993). Onboard scientific instruments such as the Wind Imaging Interferometer (WINDII), High Resolution Doppler Imager (HRDI) and the Microwave Limb Sounder (MLS) have measured the wind and temperature and revealed the tidal structures (Burrage et al., 1995; Forbes and Wu, 2006; Forbes, 2003; Hays et al., 1994; Huang and Reber, 2003; McLandress et al., 1996). The UARS satellite was decommissioned on December 15, 2005. The Thermosphere Ionosphere Mesosphere Energetics and Dynamics (TIMED) satellite is an ongoing mission launched in December 2001. The TIMED satellite provides the first systematic simultaneous measurements in temperature, density, and wind structures in the MLT by using the onboard Sounding of the Atmosphere using Broadband Emission Radiometry (SABER) and the TIMED Doppler Interferometer (TIDI) instruments (Forbes et al., 2008; Iimura et al., 2010; Ortland, 2017; Riggin and Lieberman, 2013; Wu et al., 2008; Zhang et al., 2006; Zhu et al., 2005). The Global-scale Observations of the Limb and Disk (GOLD) launched on January 25, 2018 and the Ionospheric Connection Explorer (ICON) launched on October 10, 2019 are the newest addition to the upper atmosphere observation satellites. As discussed earlier, significant changes in the ionosphere conditions are associated with energy and momentum propagating upward from the lower atmosphere. The ICON observations will be used to study the atmospheric tides in low and mid latitude regions where they are thought to be most effective in modifying the ionosphere (Cullens et al., 2020; Immel et al., 2018). One of the key objectives of GOLD is to study the effect of thermosphere temperature structure from atmospheric waves and tides propagating from below (Eastes et al., 
2017; McClintock et al., 2020).

Characterizing and understanding the causes of short-term tidal variability requires high resolution measurements on both temporal and spatial scales. Study of the tidal variability in the MLT region is complicated due to the limitations of the available observational instruments. Ground-based observations are capable of capturing the short-term variability of the atmospheric tides due to the continuous coverage of the local time. But due to the lack of spatial coverage, they are incompetent to identify the migrating and nonmigrating components of tides that are forced by different mechanisms. The different components of atmospheric tides interact differently with the mean flow and respond differently to dissipative processes such as molecular and eddy diffusion because of their vastly different vertical wavelengths. On the other hand, satellites can provide global coverage. Hence satellite tidal diagnostics can provide the climatological mean amplitudes and phases of the different tidal components. But it does not usually resolve the short-term tidal variability through the traditional linear regression method due to the slow precession of the satellite (for example, SABER needs 60 days to cover 24-hour local time). Therefore, previous research has been mainly focused on the climatology of atmospheric tides due to the lack of short-term tidal diagnostic from space-borne instruments.

Higher orbital inclinations $\left(75^{\circ}-90^{\circ}\right)$ are required to have higher latitude coverage. However, satellites generally take weeks to months to precess through 24 hours of local time in these orbits. A single satellite must tradeoff latitude coverage against local time precession rate. In order to achieve more spatial coverage and higher temporal resolution to derive short-term tidal variability, a constellation of identical satellites is needed. Currently such a system does not exist.

In recent years, there have been efforts in the satellite data analysis community to develop methods to resolve short-term tidal variability (variability $<30$ days). One of the methods is to combine data from multiple satellite instruments (Nguyen 
and Palo, 2013). The migrating diurnal tide (DW1) in the $20-80 \mathrm{~km}$ altitude range was estimated using the temperature measurements from the MLS instrument on the Earth Observing System Aura (EOS Aura) satellite and the SABER instrument on the TIMED satellite. Instrument measurement differences, poor local time sampling, and the presence of significant atmospheric oscillation other than DW1 are the major sources of error in this technique. The other one is to apply a non-spectral "deconvolution" method to a single satellite. The deconvolution method was first introduced using the CRISTA (Cryogenic Infrared Spectrometers \& Telescopes for the Atmosphere) temperatures (Oberheide et al., 2002), and has been applied successfully to temperatures from LIMS (Limb Infrared Monitor of the Stratosphere) (Lieberman et al., 2004), SABER (Oberheide et al., 2015), and winds from HRDI and WINDII (Lieberman et al., 2013). The data from SABER used in this dissertation resolve the short-term tidal variability from the second method and come from Prof. Jens Oberheide of Clemson University. The deconvolution of migrating and nonmigrating diurnal tidal components can be done by taking ascending and descending orbit node difference. The detailed method of resolving short-term DW1 will be presented in Chapter 3.

To achieve unprecedented resolution, scale, and sensitivity in the upper atmosphere and near earth space observations in the future, the decadal survey for Solar and Space Physics (2013 - 2022) by the National Research Council (2013) recommended the Dynamical Neutral Atmosphere-Ionosphere Coupling (DYNAMIC) and Geospace Dynamics Constellation (GDC) mission concepts. Dynamic will use two identical satellites separated by 6 hours in local time and $80^{\circ}$ inclination orbits at $600 \mathrm{~km}$ altitude. A major goal of the DYNAMIC mission is to characterize and understand how the lower atmosphere drives variability in the IT region. The GDC satellites will provide continued global measurements with more local time coverage. This will adequate to resolve planetary waves and tides including the resolution of ter- 
diurnal tides on a short term time basis. The primary focus of GDC is to understand how IT system regulate its response to external and meteorological forcing.

\subsubsection{Modeling Effort on Atmospheric Tides}

Physics based numerical models play a vital role in understanding and interpreting the observations of the atmospheric systems. The models provide simulations of the general circulation of the atmosphere and allow deeper insight into the physical mechanisms that drive the interactions in the atmosphere. In addition, models can simulate experiments under a more controlled environment by altering the specified parameters such as solar radiation and sea surface temperature. Models can overcome lack of temporal and spatial resolutions associated with ground-based and spaceborne measurements, hence can resolve atmospheric waves over shorter time scales. As mentioned above, lower atmospheric waves play a significant role in the MLT region. Therefore, it is necessary to develop models to realistically represent the atmosphere from the ground to the MLT region. The models that are often used studying atmospheric tides are described in the following.

The extended Canadian Middle Atmospheric Model (eCMAM) is a nonlinear time dependent model, capable of integrating the full set of primitive equations to resolve global tidal variation as a function of time. The governing equations are solved in spectral space. The eCMAM extends from ground to $\sim 220 \mathrm{~km}$. Tidal oscillations are generated self consistently from short and long wave radiation absorption, convective heating and large-scale condensation (Du et al., 2007; Fomichev et al., 2002; McLandress, 2002a,b; Ward et al., 2005). In this dissertation, the eCMAM is utilized to resolve short-term tidal variability. More details about the eCMAM are presented in Chapter 3. The Whole Atmosphere Community Climate Model with thermosphere and ionosphere extension (WACCM-X) is also a nonlinear time dependent general circulation model spanning the range of altitude from ground to the upper thermosphere 
about $500 \mathrm{~km}$. The WACCM-X uses a finite volume method to solve the governing equations. Horizontal resolution of the model is $1.9^{\circ}$ in latitude and $2.5^{\circ}$ in longitude. Vertical resolution of the model is approximately $1.1 \mathrm{~km}$ in the troposphere, $1.75 \mathrm{~km}$ at the stratopause and $3.5 \mathrm{~km}$ above $65 \mathrm{~km}$ (Liu, 2014; Liu et al., 2018). The TIMEGCM is also a nonlinear, time dependent model extending from 30 to $500 \mathrm{~km}$. The lower atmospheric forcing can be customized at the lower boundary using another model or explicitly by the user (Chang et al., 2011; Hagan and Roble, 2001; Roble and Ridley, 1994; Yamashita et al., 2010).

\subsubsection{Spatial Variability in Tides}

Tides are known to have significant temporal and spatial variability. Upon excitation, some tidal components can propagate vertically away from the source region, and their amplitudes increase exponentially as density decreases. The unique Hough modes (some are propagating modes, and some are trapped) associated with each tide based on the classical tidal equations (see Chapter 2) are the main cause for the spatial structure of each tide. The superposition of tidal components result significant

variations in the net tidal amplitude and phase as a function of longitude (see Ward et al., 2010, figure. 1).

Figure 1.4 shows the complex spatial structures in the climatological mean of the zonal wind components for different diurnal tides. Climatological mean of the zonal wind amplitudes are calculated from the eCMAM30 data between $1979-2010$. The migrating diurnal tide DW1 (top left) amplitudes of the zonal wind exhibit two amplitude maxima which are symmetric about the equator and are located between 0 and $50^{\circ} \mathrm{N} / \mathrm{S}$ and centered around $30^{\circ} \mathrm{N} / \mathrm{S}$, respectively. The maximum amplitudes in both hemispheres are greater than $25 \mathrm{~m} \mathrm{~s}^{-1}$ between $\sim 90-100 \mathrm{~km}$. The zonal amplitudes decrease with the altitudes above $100 \mathrm{~km}$. In addition to these two subtropical maxima, there are two maxima located at polar regions of both hemispheres 
above $100 \mathrm{~km}$ with significant amplitudes $\left(>10 \mathrm{~m} \mathrm{~s}^{-1}\right)$, which are probably excited locally by the EUV absorption in this region. The nonmigrating eastward diurnal tide DE3 (top right) shows a broad single maximum between $30^{\circ}-30^{\circ} \mathrm{N} / \mathrm{S}$. The equatorial maximum reaches over $25 \mathrm{~m} \mathrm{~s}^{-1}$ above $100 \mathrm{~km}$. The maximum climatological amplitude is larger than that of the DW1 component at the equatorial region. The climatology for the nonmigrating westward diurnal tide DW2 (bottom left) zonal wind component shows a broad feature located between $45^{\circ} \mathrm{N} / \mathrm{S}$ and between $80-110 \mathrm{~km}$. The maximum amplitudes are located at $20^{\circ}-30^{\circ} \mathrm{N} / \mathrm{S}$. The two maxima merge into one over the equator above $95 \mathrm{~km}$ and the maximum amplitudes are about $9 \mathrm{~m} \mathrm{~s}^{-1}$. The zonal wind component of the nonmigrating stationary diurnal tide DS0 (bottom right) shows that there is a maximum between $80-110 \mathrm{~km}$ in each hemisphere, which are symmetric about the equator. They are located between 0 and $50^{\circ} \mathrm{N} / \mathrm{S}$ and centered on $30^{\circ} \mathrm{N} / \mathrm{S}$. The southern hemisphere maximum has slightly stronger amplitudes than its northern hemisphere counterpart. The maximum amplitude in the southern hemisphere is over $11 \mathrm{~m} \mathrm{~s}^{-1}$ and $\sim 95 \mathrm{~km}$. The northern hemisphere maximum amplitude is located above $100 \mathrm{~km}$ and $\sim 9 \mathrm{~m} \mathrm{~s}^{-1}$.

In the equatorial MLT region, the migrating diurnal tide DW1 (westward propagating zonal wavenumber 1 with a period of $24 \mathrm{~h}$ ) is arguably the largest and most persistent atmospheric tide in the temperature and wind fields (Mukhtarov et al., 2009). Thus employ DW1 as the primary tide in this research. The DW1 is mainly excited by $\mathrm{H}_{2} \mathrm{O}$ solar radiation absorption in the troposphere (Forbes, 1982b), yet absorption of solar radiation by ozone in the stratosphere (Hagan, 1996) and tropospheric latent heating (Forbes et al., 1997) also make non-negligible contributions. Gan et al. (2014) presented a detailed comparison of the DW1 climatological altitudinal-latitudinal structure, seasonal variability, and interannual variability between eCMAM and SABER. The latitudinal structure of DW1 in temperature is characterized by maxima located in the mid and high latitudes between $40-60 \mathrm{~km}$ 

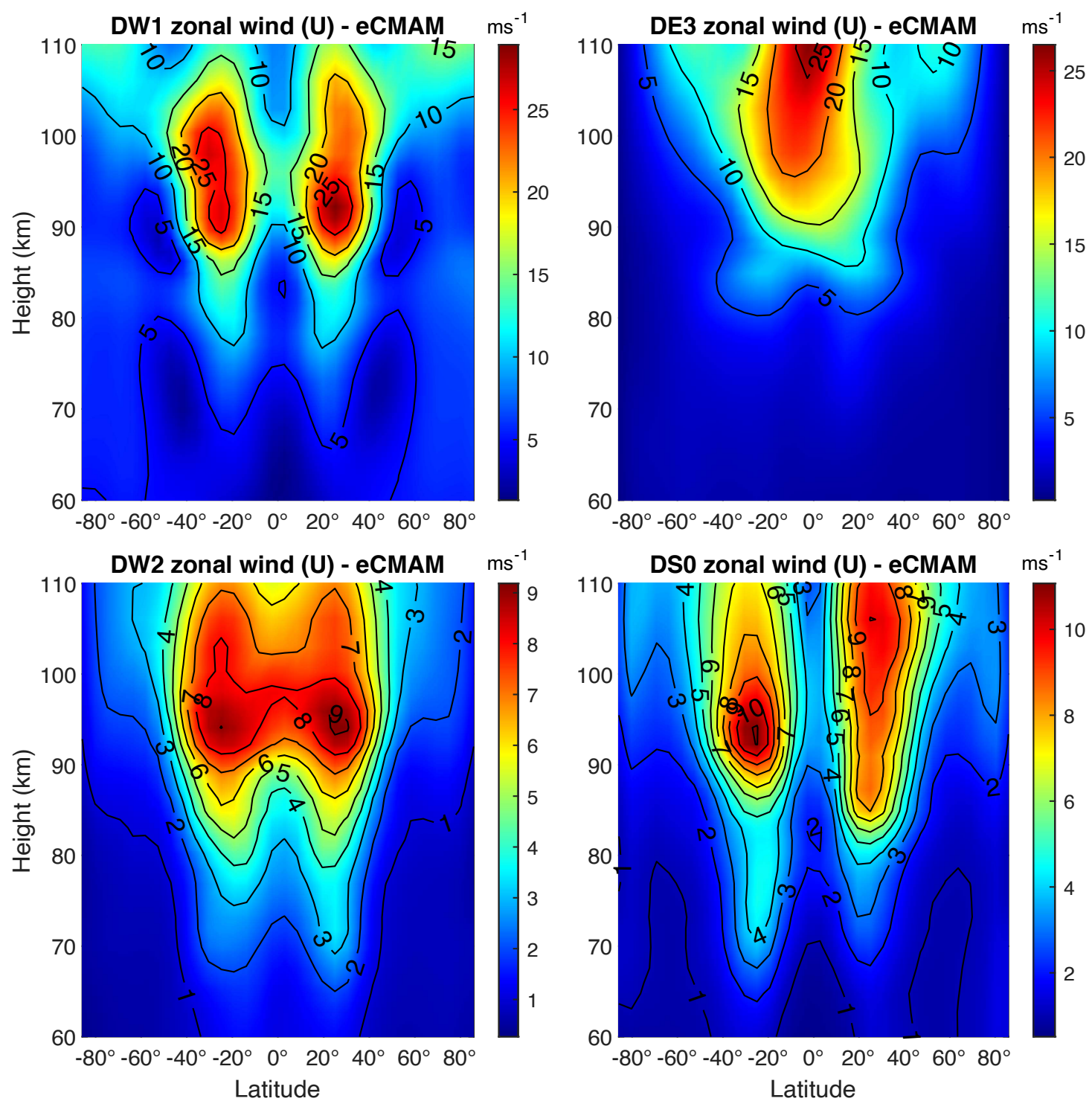

Figure 1.4. Climatological mean of the zonal wind amplitude $\left(\mathrm{m} \mathrm{s}^{-1}\right)$ of the diurnal tides: DW1, DE3, DW2 and DS0 as a function of latitude and height in the MLT region. 
in each hemisphere. DW1 is trapped and cannot propagate upward in this region due to in situ excitation by $\mathrm{O}_{3}$ absorption of UV solar radiation (Forbes et al., 2006; Forbes and Garrett, 1979; Forbes and Wu, 2006; Zhang et al., 2006). Above 60 km, the altitude-latitude structure of DW1 is characterized by an equatorial maximum and two secondary maxima located symmetrically in the mid-latitudes (near $\pm 35^{\circ}$ ) of each hemisphere. DW1 in this region is propagating upward with vertical wavelength about $20-25 \mathrm{~km}$ (Forbes and Wu, 2006; Smith, 2012; Zhang et al., 2006), and DW1 starts to dissipate above $96 \mathrm{~km}$ (Lu et al., 2011). While DW1 temperature maximizes at the equator, the latitudinal structure of DW1 in meridional wind maximizes in the subtropics (Singh and Gurubaran, 2017).

Nonmigrating tides can be excited by land-sea contrast, longitudinal variation in diurnal heating (Kato et al., 1982; Tsuda and Kato, 1989), latent heat release in the tropical troposphere (Hagan et al., 2009; Hagan and Forbes, 2002), nonlinear tide-tide interaction (Teitelbaum and Vial, 1991; Smith and Ortland, 2001), and tide-quasi-stationary planetary wave (SPW) interaction (Hagan and Roble, 2001; Yamashita et al., 2002; Angelats i Coll and Forbes, 2002; Lieberman et al., 2004; Grieger et al., 2004). DE3, D0, DW2, and DW5 are the significant nonmigrating diurnal tidal components in the MLT region. D0 and DW2 are mostly generated by nonlinear interaction between DW1 and SPW1 (stationary planetary wave with zonal wavenumber 1) (Grieger et al., 2004; Hagan and Roble, 2001; Lieberman et al., 2004; Mayr et al., 2005a,b). The maximum amplitudes for DW2 meridional wind occur during October-November and January-February (Lu et al., 2011). DE3 and DW5 are primarily excited by latent heating associated with deep tropical convection (Forbes et al., 2001; Hagan and Forbes, 2002). Oberheide et al. (2006) analyzed the zonal and meridional winds measured by TIDI and presented climatologies of monthly mean amplitudes and phases for seven nonmigrating diurnal tidal components between 85 and $105 \mathrm{~km}$ altitude and latitude between $45^{\circ} \mathrm{N} / \mathrm{S}$ (DE3, DE2, DE1, 
DS0, DW2, DW3, and DW4). Zhang et al. (2012) studied the seasonal-latitudinal variation of the DE3 and showed that the seasonal-latitudinal variation of mean zonal wind modulates and filters DE3 energy propagating upwards from tropospheric radiative and latent heating sources. The strongest DE3 occurs primarily during the northern hemisphere's summer/fall with maximum eastward winds near the equator (Forbes, 2003).

Semidiurnal tides are dominating over diurnal tides at latitudes higher than $45^{\circ}$ N/S in the MLT region (Vincent et al., 1989; Yi, 2001). The migrating semidiurnal tide SW2 is mainly excited by the absorption of solar radiation by ozone in the stratosphere and lower mesosphere (Forbes, 1982a). Semidiurnal tides have smaller amplitudes and seasonally symmetric phases in mid and low latitudes compared to the tidal structures above $50^{\circ} \mathrm{N} / \mathrm{S}$ (Forbes and Vial, 1989). Observational and modeling studies both suggest that the seasonal structure of the semidiurnal tidal wind field in the MLT region is dominated by large amplitudes in winter and late summer/early autumn (Jacobi et al., 1999). The amplitude modulations of the semidiurnal tide observed in the MLT region during winter are mainly produced by non-local coupling between the semidiurnal tide and planetary waves in the stratosphere (Pancheva et al., 2003). In the mid-latitude region, the main tidal component is the SW2, which has amplitudes reaching over $20 \mathrm{~m} \mathrm{~s}^{-1}$ for both zonal and meridional winds. Observational and numerical studies have shown that the SW2 and lunar semidiurnal tide at the mesosphere and thermosphere altitudes enhances during the SSWs (Fuller-Rowell et al., 2010; Jin et al., 2012).

Du et al. (2007) conducted a detailed investigation about migrating and nonmigrating semidiurnal tides simulated from eCMAM and compared the mean climatology, latitudinal structures, and seasonal variation with TIDI wind measurements. Significant nonmigrating semidiurnal tidal components in the MLT are SE2, SW1, SW3, and SW6. SE2 and SW6 forced by latent heating associated with deep tropical 
convection (Hagan and Forbes, 2003), whereas SW1 and SW3 are thought to be excited by nonlinear interaction between SW2 and SPW1 (Angelats i Coll and Forbes, 2002; Yamashita et al., 2002). Iimura et al. (2010) assessed the spatial structure and temporal evolution of the nonmigrating semidiurnal tides over the Arctic from the TIDI wind measurements and compared the structure of the nonmigrating semidiurnal tide between the Arctic and Antarctic. The nonmigrating semidiurnal tidal wind field over the Arctic comprises mainly SW1, SW3, and SS0. The SW1 tide is the most prominent, maximizing above $90 \mathrm{~km}$ poleward of $60^{\circ} \mathrm{N}$ during mid-March to mid-May.

In contrast to the extensive studies on the characteristics of the diurnal and semidiurnal tides, the terdiurnal tide has received less attention because of their relatively small amplitude. However, some studies have shown that the amplitude of terdiurnal tide can be large as the ones of diurnal and semidiurnal (Cevolani, 1987; Jacobi, 2012; Reddi et al., 1993; Thayaparan, 1997; Younger et al., 2002). Early work done by Glass and Fellous (1975) shows the existence of terdiurnal tide using the measurements from meteor radars at Garchy $\left(47^{\circ} \mathrm{N}\right)$. Teitelbaum et al. (1989) performed a more detailed analysis of the terdiurnal tidal structure using the data from Garchy and Montpazier $\left(44^{\circ} \mathrm{N}\right)$ in comparison with terdiurnal tide observed over Saskatoon $\left(52^{\circ} \mathrm{N}\right)$ and Budrio $\left(45^{\circ} \mathrm{N}\right)$. They showed that the superposition of the diurnal solar heating and the nonlinear interaction between the diurnal and semidiurnal tide could produce the observed terdiurnal tide during the summer months. Several different excitation mechanisms for the migrating terdiurnal tide TW3 have been proposed. These include: direct thermal excitation by solar heating, primarily in the upper stratosphere (Chapman and Lindzen, 1970), nonlinear interaction between the 12and 24-hour tides (Smith, 2012), a combination of the above two (Akmaev, 2001; Glass and Fellous, 1975; Teitelbaum et al., 1989), and interaction of the 24-hour tide with gravity waves (Miyahara and Forbes, 1991). Du and Ward (2010) presented a 
detailed analysis of annual mean structure, seasonal variations, vertical wavelengths and superposition of the terdiurnal tides using migrating (TW3) and nonmigrating (TW1, TW2, TS0 and TE5) components from the eCMAM. Pancheva et al. (2013) presented a detailed study of the climatology of the migrating terdiurnal tide (TW3) in the latitude range of $\pm 50^{\circ}$ and altitudes between 60 and $110 \mathrm{~km}$ using the SABER temperature measurements and the WACCM model. It is found that the SABER TW3 tide is dominated by different Hough modes below and above $\sim 80$ to $90 \mathrm{~km}$ height. Liu et al. (2019) studied the terdiurnal tide in the Antarctic MLT region using the observation over Syowa $\left(69^{\circ} \mathrm{S}, 39^{\circ} \mathrm{E}\right)$ during years of $2004-2018$. This study revealed that the vertical structures largely differ from those in the Arctic, indicating hemispheric asymmetry. Analysis of a decade long climatology of terdiurnal tides from SABER observations, Moudden and Forbes (2013) suggest that tidal components TE1, TW4, and TW5 vary in a way that they originate from nonlinear interactions between diurnal and semidiurnal tides (between DE3 and SW2 for TE1, between DW2 and SW2 for TW4, and between DW1 and SW4 for TW5). An interaction between two primary waves with frequencies $n_{1} \Omega$ and $n_{2} \Omega$ and zonal wave numbers $s_{1}$ and $s_{2}$, results in the generation of secondary waves with the "sum and difference" frequencies $\left(n_{1} \Omega+n_{2} \Omega, n_{1} \Omega-n_{2} \Omega\right)$ and zonal wave numbers $\left(s_{1}+s_{2}\right.$, $\left.s_{1}-s_{2}\right)$ (Teitelbaum and Vial, 1991).

\subsubsection{Temporal Variability in Tides}

The temporal variability of a single tidal component has been observed to occur on time scales ranging from days to years. Here tidal climatology is defined as variability associated with periods longer than 30 days, whereas tidal weather is shorter than 30 days (variability on planetary wave time scale). The tidal climatology can be further separated into inter-annual ( $>12$ months), seasonal (3 - 12 months), and intra-seasonal (1-3 months) variabilities. 
The interannual variability of the tides is mainly linked to solar variability, El Niño-Southern Oscillation (ENSO), and the stratospheric Quasi-biennial Oscillation (QBO). The solar cycle is a nearly periodic 11-year change in the Sun's magnetic field activity. The increased solar activity includes increasing extreme ultraviolet and X-ray emissions from the Sun that produce dramatic effects in Earth's upper atmosphere. The variability of the solar UV spectral irradiance affects the thermal structure of the atmosphere by directly changing the total energy deposited and indirectly modifying the photochemistry and dynamics of the atmosphere. The EUV short-wave solar heating generates DW1 in-situ, which is locally trapped with a coherent phase throughout the thermosphere. The thermosphere expands, and EUV radiation intensifies with increasing solar activity, so DW1 amplitudes increase with solar cycle (Hagan and Roble, 2001). Singh and Gurubaran (2017) suggested solar cycle variation in diurnal tide using ground-based MF radar measurements located at Tirunelveli $\left(8.7^{\circ} \mathrm{N}, 77.8^{\circ} \mathrm{E}\right)$. Oberheide et al. (2009) studied the temperature, density, horizontal and vertical winds for DE3 in MLT and upper thermosphere during solar maximum to solar minimum $(2002-2008)$ using the TIMED and CHAMP (Challenging Minisatellite Payload) satellite data and has reported no DE3 solar cycle dependence in the MLT region.

Moreover, Nischal et al. (2019) studied the solar cycle variation of nonmigrating tides DE3 and DE2 using NO $5.3 \mu \mathrm{m}$ and $\mathrm{CO}_{2} 15 \mu \mathrm{m}$ IR cooling rates from SABER data during 2002 - 2013. NO cooling rate in tidal amplitude shows a significant solar cycle variation, and the $\mathrm{CO}_{2}$ cooling rate shows a weak solar cycle dependence. Zhou et al. (2016) used electron density observations from CHAMP and GRACE (Gravity Recovery and Climate Experiment) satellites to investigate the solar activity dependence of nonmigrating tides at both low and middle latitudes. The absolute amplitudes of DE3 at the low latitudes and DE1, D0, and DW2 at middle latitudes are highly related to the solar activity, all showing larger amplitudes during solar 
maximum years. Luan et al. (2012) reported the solar cycle variations of terdiurnal migrating tide (TW3) signature in ionospheric total electron content.

The QBO is a tropical, lower stratospheric, downward propagating equatorial zonal wind variation between easterlies and westerlies, with a variable period averaging $\sim 28$ months. The Spectrum analysis over more extended time series from SABER and eCMAM has also revealed a variability with a period of $25-26$ months similar to the QBO in stratospheric zonal winds, which indicates the modulation of the QBO on DW1 (Gan et al., 2014). DW1 tidal amplitude is generally stronger/weaker during the westerly/easterly phase of the QBO (Smith, 2012). Analysis from ground-based radar and satellite observations indicate that DW1 exhibits a strong interannual variation closely related to the stratospheric QBO and the DW1 amplitude reaches maximum during March/April of years when the stratospheric QBO in eastward phase and weaker during westward phase (Burrage et al., 1995; Hagan et al., 1999b; Lieberman, 1997; Vincent et al., 1998; Wu et al., 2008). Strong QBO signatures can be found in the amplitude of the diurnal tidal temperature in the tropical region and in the wind near $20^{\circ} \mathrm{N} / \mathrm{S}$ (Xu et al., 2009). Mayr and Mengel (2005) found that the QBO can modulate seasonal maximum amplitude in diurnal tide as much as $30 \%$ from one year to another and discussed the underlying mechanisms using linearized advection terms. Forbes et al. (2008) found a QBO variation of order $\pm 10-15 \%$ in the DW1 and SW2 tidal amplitudes in temperature measurements obtained by the SABER between 100 and $116 \mathrm{~km}$. From the zonal wind observations at $105 \mathrm{~km}$, similar QBO variations in the DE3 tidal amplitude have also been reported by Oberheide et al. (2009). At low latitudes, a clear modulation by the $\mathrm{QBO}$ can be found in the relative amplitudes of DE3 from both CHAMP and GRACE satellite observations (Zhou et al., 2016). The interannual variability of the SW2 tide in the midlatitude lower thermosphere is at least partly connected with the stratospheric QBO as this effect is stronger in the NH (Pancheva et al., 2009; Dhadly et al., 2018). The TW3 revealed some inter- 
annual variability with a period of quasi-2 years and indicates a clear enhancement during the eastward phase of the stratospheric QBO (Pancheva et al., 2013). Kumari and Oberheide (2020) studied the interannual changes in short-term DE3 variability on planetary wave timescales and found that symmetric DE3 mode variability is enhanced during the easterly phase of the QBO due to enhanced quasi-10-day wave activity and during La Niña phase of ENSO due to enhanced convective forcing.

ENSO is a recurring climate pattern involving changes in the temperature of waters in the central and eastern tropical Pacific Ocean. This oscillating warming and cooling pattern on periods ranging from about three to seven years referred to as the ENSO cycle. ENSO is one of the most important climate phenomena on Earth due to its ability to change the global atmospheric circulation, which in turn, influences temperature and precipitation across the globe. El Niño (winds cause warm surface water from the equator to move east, toward Central and South America) and La Niña (strong winds blow warm water at the ocean's surface from South America to Indonesia) are the extreme phases of the ENSO cycle. With large variations in the rainfall,latent heat patterns, radiative heating in the water vapor, and changed cloud coverage caused by ENSO, noticeable effects on the atmospheric tidal variability in the MLT region have been reported by several studies (Fernández et al., 2004; Gurubaran, 2005; Lieberman et al., 2007, 2015; Liu et al., 2017; Vial et al., 1994; Pedatella and Liu, 2012, 2013b; Warner and Oberheide, 2014). WACCM simulations indicate that ENSO represents $\sim 10-30 \%$ inter-annual tidal variability in MLT during the northern hemisphere winter. DW1 and SW4 are enhanced during the El Niño phase of the ENSO while DE3 and DE2 are decreased (Pedatella and Liu, 2012, 2013b). DE3 and DE2 tidal wind variability from TIDI/TIMED revealed that the tidal response to ENSO is largest during winter. The DE3 tidal response to the La Niña phase increased $\sim 70 \%$ for the winter month in 2010/2011 (Warner and Oberheide, 2014). 
Sun et al. (2018) showed that the QBO in DW1 amplitudes near the equator and DE3 amplitudes at low latitudes has shorter periods ( $\sim 2$ years) during the ENSO warm phase whereas longer periods ( $\sim 2.5$ years) during ENSO neutral and cold phases using observations from SABER and GAIA (Ground-to-topside model of Atmosphere and Ionosphere for Aeronomy) simulations. The signal of the QBO in the extratropical stratosphere tends to be strengthened in solar minima, and weakened in solar maxima (Labitzke and Loon, 1988). Therefore, the study of interannual variability needs to consider the composite influence of the solar cycle-QBO-ENSO on tidal amplitudes.

Strong seasonal variations in DW1 have been documented from winds observed by HRDI (Burrage et al., 1995; Huang and Reber, 2003), by WINDII (McLandress et al., 1996), and variations in the temperature observed by SABER (Xu et al., 2009; Zhang et al., 2006), long-term MF radar wind measurement by Vincent et al. (1998) and Fritts and Isler (1994). DW1 exhibits a strong semiannual variation which is characterized by an equinoctial maximum and summer minimum in the MLT region and shows equinoctial asymmetry with larger amplitudes in spring equinox than in fall equinox. Proposed explanations for the semiannual variation of DW1 are semiannual variations in the heating that force tide (Hagan and Forbes, 2002; Lieberman et al., 2003; Xu et al., 2010), variations due to dissipation within the MLT (Lieberman et al., 2010; Xu et al., 2009), and variation of the background winds in the tropical stratosphere and mesosphere (McLandress, 2002a,b). In the stratosphere, DW1 exhibits an annual variation and maximizes in the summer of each hemisphere. The annual variation of DW1 is mainly governed by short-wave heating and linear advection (Gu and Du, 2018). Svoboda et al. (2005) reported terannual variability in DW1 meridional wind in the northern hemisphere. DW1 in the thermosphere is characterized by hemispheric annual variation and maximizes during summer of each hemisphere ( $\mathrm{Gu}$ and Du, 2018; Jones et al., 2016). The seasonal variation in DE3 at $105 \mathrm{~km}$ is 
dominated by an annual variation with amplitude reaching maximum $(12 \mathrm{~K})$ between June and October and minimum between December and March. DW2 exhibits a semiannual variation at $105 \mathrm{~km}$ with maximum in March and October and minimum in January, June, and July in eCMAM (Gan et al., 2014). The migrating semidiurnal tidal (SW2) shows larger amplitudes $(\sim 20 \mathrm{~K})$ in January, May-June and August At above $90 \mathrm{~km}$ (Sridharan, 2019). The seasonal behavior of the migrating semidiurnal tide in the middle latitudes $\left( \pm 40^{\circ}\right)$ is dominated by annual variability with a winter maximum in the upper mesosphere $(90 \mathrm{~km})$ of both hemispheres and summer one in the lower thermosphere $(110 \mathrm{~km})$ (Pancheva et al., 2009). The seasonal behavior of the migrating terdiurnal tide TW3 at low latitudes is dominated by an annual variation with local summer amplification, and at midlatitudes, it is a combination of semiannual variation with maxima in equinoxes and an annual variability with winter maximum (Pancheva et al., 2013).

Intra-seasonal cycles in deep convection can be characterized as the Madden-Julian Oscillation (MJO) (Madden and Julian, 1971). The MJO is an eastward moving disturbance of clouds, rainfall, winds, and pressure near the equator that typically recurs every 30 to 60 days mainly over the Indian and Pacific Ocean. Vergados et al. (2018) found dominant periodicities at 40 - 60 days in MLT temperature variations during MJO season (November-April) and suggest that atmospheric tides modulated by MJO lead this variation. Yang et al. (2018) investigated the response of the mesospheric migrating diurnal tide (DW1) to the MJO using a simulation from the Specified-Dynamic Whole Atmosphere Community Climate Model (SD-WACCM). Their analysis showed that a significant connection exists between the MJO and the mesospheric DW1 tidal amplitude. Upward propagation of nonmigrating tides modulated by the MJO can leave imprints at the same period over a broad range of vertical levels (Eckermann et al., 1997; Lieberman, 1998). Gasperini et al. (2017) reported a 90-day global scale oscillation in DE3 and thermospheric zonal wind and 
suggested that strong coupling between the troposphere and the thermosphere occurs on intra-seasonal time scales. Observations from the MLS/AURA satellite between $2004-2017$ revealed significant intra-seasonal variability (30 - 60 days) in the wave-3 and wave-4 longitudinal structures near the mesopause (Liu et al., 2018).

Both observations and numerical simulations have revealed significant tidal weather (e.g., short-term tidal variability with time scale of $\sim 5$ to 30 days) associated with tides. Only a handful of studies have focused on short-term tidal variability due to the lack of spatial coverage from ground-based observations and the insufficient time resolution of satellite data. Hence up to today, we know very little about how these variability evolves with time and space and the physical mechanisms governing these variability.

Using five years of wind data from a radar system at Christmas Island $\left(2^{\circ} \mathrm{N}, 157^{\circ}\right.$ W), Eckermann et al. (1997) showed that there are strong variations in diurnal tidal amplitudes with a period of $\sim 24$ days. From the Kyushu University general circulation model, Miyoshi and Fujiwara (2003) noted fluctuations of the DW1 amplitude with a period of 25 days in the height range from 20 to $300 \mathrm{~km}$. A modeling study by Liu (2014) with the WACCM-X noted that the amplitudes of DW1 (periods smaller than 10 days) vary significantly from one day to the next, the time scale is shorter than the time scales associated with planetary wave variability (weeks to a month), and this variability is ubiquitous and does not show any clear seasonal, annual, or inter-annual dependence (planetary waves usually do). She et al. (2004) and Liu et al. (2007) showed from lidar measurements that the diurnal temperature amplitude in the MLT region can be double or even triple from one day to the next. Chang et al. (2011) found that nonlinear interaction between the migrating diurnal tide DW1 and the quasi-two-day wave (QTDW) can decrease tidal amplitude about $20-40 \%$ in the MLT region. The TIMED Doppler Interferometer and Navy Global Environmental Model exhibits significant short-term variability in DW1 and tidal amplitude in 
DW1 is found to be $\sim 64 \%$ change due to the short-term variability (Dhadly et al., 2018). Pedatella et al. (2012a) suggest that day to day variability in DW1 might have been due to the nonlinear interaction with the 6-day planetary wave. Pedatella et al. (2016) investigate the impact of the DE3 short-term tidal variability in the MLT region using SABER and WACCM+DART (Whole Atmosphere Community Climate Model + Data Assimilation Research Testbed) and revealed tidal amplitude changes of $5-10 \mathrm{~K}$ on short time scales $(\sim 10-20$ days $)$. Short-term tidal variability may also account for the observed upward transmission of planetary wave periodicities into the ionosphere. For example, during the SSW events, the ionosphere/thermosphere shows large short-term variability in response to lower atmosphere changes (Chau et al., 2012; Goncharenko et al., 2012; Goncharenko and Zhang, 2008).

The exact causes of the tidal day-to-day variability in the MLT have not been systematically studied, but there are several plausible ones:

1. Variations in the background wind: Tidal propagation is dependent on the background winds along its path from the source regions to the MLT (Ekanayake et al., 1997; Hagan et al., 1999a).

2. Variability in the tidal sources: variations in the distribution of water vapor and stratospheric ozone may also cause short-term variability (Kumar et al., 2008; Lieberman et al., 2004; Oberheide et al., 2002; Pedatella et al., 2016; Yi, 2001).

3. Non-linear interaction: Atmospheric tides have been regularly observed to fluctuate on planetary wave time scales and this has been interpreted as interactions between the tides and planetary waves (Angelats i Coll and Forbes, 2002; Liu et al., 2007; Miyahara et al., 1999; Pancheva et al., 2002; Teitelbaum and Vial, 1991). 
Teitelbaum and Vial (1991) proposed that nonlinear coupling between the migrating tides and stationary planetary waves can also generate nonmigrating tides, which are child waves with frequencies and wavenumbers equal to the sum and difference of those of the parent waves. Observed correlations between the migrating tides and planetary wave activity provide evidence for this mechanism (Forbes, 1995; Talaat and Lieberman, 1999; Pancheva et al., 2002) and modeling studies have also shown that this interaction can produce nonmigrating tides with large amplitudes (Miyahara et al., 1999; Hagan and Roble, 2001; Angelats i Coll and Forbes, 2002; Liu et al., 2007).

\subsection{Objectives}

As described above, the present understanding about impacts from atmospheric tides to the ionosphere-thermosphere (IT) region is limited to the seasonal e.g., "climatological" timescale. Thus, how the atmospheric tides lead to day-to-day variability in the IT region pose an important challenge to be addressed in order to understand the meteorological driving of the IT System. The overall objective of this dissertation research is to make the step from "seasonal/climatological tides" to "tidal weather (tidal variability less than 30 days)." This study is used data from a state-of-art general circulation model: the eCMAM and space-borne instrument: SABER to study the short-term tidal variability. The research will focus on one of the atmospheric tides: the migrating diurnal tide (DW1) in the mesosphere and lower thermosphere region $(\sim 60-120 \mathrm{~km})$. We will study the statistical properties of short-term tidal variability, perform model and observation comparisons, develop forecast models for short-term tidal variability and total tidal variability, and investigate possible physical mechanisms of short-term tidal variability. To our knowledge, this is the first systematic study of the short-term tidal variability of this kind.

Particularly, the following questions will be addressed in this dissertation: 
1. What are the statistical characteristics of the DW1 short-term tidal variability and can we model and forecast the short-term tidal variability based on the statistical properties?

The lack of knowledge and short term tidal diagnostics are the main challenges to understanding the short-term tidal variability as a function of latitude, height and time. To address this question, first we need to understand the statistical properties of short-term tidal variability. How does it change with time, is there any self memory, or is it totally stochastic? How does it change spatially, are (or to what extent) they related to their neighboring latitudes/heights? Then based on the temporal and spatial statistical properties, e.g., the auto- and spatial correlations, a hierarchy of statistical models can be developed to predict the short-term tidal variability. Finally, prediction accuracy of the models and their stability will be evaluated with different sensitivity tests and compared between the eCMAM and SABER.

2. How does the total tidal variability of DW1 response to the interannual and seasonal oscillations, are there any imprints of these oscillations in short-term tidal variability, and how is the relative importance of the short-term, seasonal, and interannual tidal variability?

The DW1 total variability from both the model and observation will be fitted into a multiple linear regression model accounting for the solar cycle, ENSO, QBO, and seasonal harmonics as a function of latitude and height. There has been very little research on long-term (intraseasonal, seasonal, and interannual) variations of short-term tidal amplitudes in the MLT region. This study will examine the long-term variations in short-term tidal variability and quantify the relative importance of short-term tidal variability relative to the intraseasonal, seasonal, and inter annual time scales. Finally, total tidal variability will be 
predicted based on the multiple linear regression model and the tidal weather forecast model developed for question 1.

3. What are the underlying physical mechanisms of short-term tidal variability? There are three possible sources for the short-term tidal variability: variability in the tidal generation source, interactions with background wind, and nonlinear interactions with other waves. To examine the underlying physical mechanisms of short-term tidal variability, the thermodynamic budget terms will be investigated including linear advection (wave-mean flow interaction), nonlinear advection (wave-wave interaction) and short-wave solar heating (tidal generation source) from the model (eCMAM) to quantify their relative importance.

\subsection{Thesis Outline}

The content of the dissertation following this introductory chapter is organized into 6 main chapters. In Chapter 2, the theoretical background on the dynamics of the atmospheric tides pertaining to the focus of this dissertation will be provided. The contents of Chapter 2 are mainly summarized from the well-renowned atmosphere dynamics textbooks of (Holton, 1975, 2004), and the classical tidal theory attributed to Chapman and Lindzen (1970).

Chapter 3 of this dissertation introduces the data analysis methods and describes the eCMAM and SABER data. Chapter 3 also discusses how the short-term tidal variability is extracted from SABER and how to fill the missing data due to the temporal gaps in the satellite measurements.

Chapter 4 focuses on the short-term tidal variability. This chapter presents the published results from Vitharana et al. (2019). First section illustrates the statistical properties of tidal weather, e.g., the auto- and spatial correlations. Based on these statistical properties of tidal weather, a hierarchy of statistical models are developed 
to predict the tidal weather. Different sensitivity tests are performed on the statistical models to make sure the coefficients used in these models are robust. Tidal weather forecast from the statistical models based on coefficients obtained from eCMAM and SABER are compared at the end of Chapter 4.

Chapter 5 examines the total variability of DW1. A multiple linear regression model is fitted to both the eCMAM and SABER, accounting for the solar cycle, ENSO, QBO, and seasonal harmonics, and their corresponding coefficients are compared between the model and observations. This chapter then describes the long-term variability presented in the short-term tidal variability and investigates the relative importance of short-term, seasonal, and interannual variability. The total tidal variability is predicted based on the multiple linear regression model and the tidal weather forecast model from Chapter 4 . The best and worst prediction years are discussed and possible causes explored.

Chapter 6 focuses on the physical mechanisms governing the short-term tidal variability in the mesosphere and thermosphere. Possible mechanisms that govern the short-term tidal variability include the linear advection (wave-mean flow interaction), nonlinear advection (wave-wave interaction) and short-wave solar heating. All these terms can be obtained from the thermodynamic budget in the eCMAM. Non-local effects from solar heating are also studied.

Finally, Chapter 7 presents the conclusions for this dissertation, and a list of possible future work. 


\section{CHAPTER 2}

\section{BASIC DYNAMICS OF THE ATMOSPHERE}

Describing the governing dynamics of the atmosphere is not a straightforward task due to the complex nature of the atmosphere from both the thermal and mechanical perspective. However, with appropriate approximations and assumptions, a set of primitive equations can be produced to explain the governing physics in the atmosphere. Most of the major developments in the atmosphere dynamics were accomplished in the past 70 years. This chapter presents the theoretical background pertaining to the focus of this dissertation. The contents of this chapter are mainly summarized from the well-known atmosphere dynamics textbooks of Holton (2004, 1975), classical tidal theory attributed to Chapman and Lindzen (1970), and thermodynamic budget analysis from $\mathrm{Gu}$ and $\mathrm{Du}$ (2018). The present chapter will not provide a comprehensive review. Instead, it will describe the equations relevant to the description of atmospheric tides with approximations and assumptions along the way. References have been kept to a minimum in this chapter. More detailed reviews and references can be found in the textbooks above.

\subsection{Primitive Equations}

The primitive equations may describe the motions of the atmosphere. The primitive equations are derived from the Navier-Stokes equations for a compressible gas, which

consists of the horizontal momentum equations, the first law of thermodynamics, the 
continuity equation, and the hydrostatic approximation. These primitive equations form the basis of general circulation models that have been used to simulate the atmospheric circulation. In their complete form, these equations are highly nonlinear and far more complicated. However, to gain a physical understanding of the qualitative nature of the various dynamical phenomena, it is useful to apply simplifications such as dynamical simplifications, geometrical simplifications, and linearization to the governing equations. In particular, the variable distance to the center of Earth is replaced by a constant mean Earth radius, the Coriolis force terms associated with the horizontal component of the rotation vector are omitted, and the vertical momentum equation can be replaced by hydrostatic balance. Throughout this work, we will generally use the log-pressure height $\mathrm{z}$ as the vertical coordinate define by

$$
z=-H \ln \left(\frac{p}{p_{s}}\right)
$$

where $p$ is pressure, $p_{s}$ is the surface pressure (usually taken as 1000 mbar or $\left.10^{5} \mathrm{~Pa}\right)$, and $H$ is the mean scale height $\left(=R T_{s} / g_{0}\right.$, where $R$ is the gas constant for dry air and equals to $287 \mathrm{~J} \mathrm{~K}^{-1} \mathrm{~kg}^{-1}, T_{s}$ is a constant reference temperature, $g_{0}=9.80665 \mathrm{~m} \mathrm{~s}^{-2}$ is the global average of gravity at mean sea level).

Primitive equations may be expressed using the log-pressure height $\mathrm{z}$ as the vertical coordinate and spherical coordinates as follows (Holton, 1975).

$$
\begin{gathered}
\frac{D u}{D t}-\left(2 \Omega \sin \phi+u \frac{\tan \phi}{a}\right) v+\frac{1}{a \cos \phi} \frac{\partial \Phi}{\partial \lambda}=X \\
\frac{D v}{D t}+\left(2 \Omega \sin \phi+u \frac{\tan \phi}{a}\right) u+\frac{1}{a} \frac{\partial \Phi}{\partial \phi}=Y \\
\frac{D \theta}{D t}=Q
\end{gathered}
$$




$$
\begin{gathered}
\frac{1}{a \cos \phi}\left(\frac{\partial u}{\partial \lambda}+\frac{\partial v \cos \phi}{\partial \phi}\right)+\frac{1}{\rho_{o}} \frac{\partial\left(\rho_{o} w\right)}{\partial z}=0 \\
\Phi_{z}=\frac{\partial \Phi}{\partial z}=\frac{R \theta e^{-\kappa z / H}}{H}
\end{gathered}
$$

where the material derivative $D / D t$ is given by

$$
\frac{D}{D t}=\frac{\partial}{\partial t}+\frac{u}{a \cos \phi} \frac{\partial}{\partial \lambda}+\frac{v}{a} \frac{\partial}{\partial \phi}+w \frac{\partial}{\partial z}
$$

$\phi \quad$ latitude

$\lambda$ longitude

$\Phi$ departure of local geopotential

$\rho_{o} \quad$ a basic state density $\left[\equiv \rho_{s} e^{-z / H}\right]$

$Q$ heating rate

a radius of Earth

$\Omega$ angular velocity of the Earth $7.292 \times 10^{-5} \mathrm{~s}^{-1}$

$u$ eastward velocity

$v$ northward velocity

$w$ a measure of vertical velocity $\left[\equiv \frac{d z}{d t}\right]$

$\kappa$ ratio of the gas constant to specific heat at constant pressure $\left[\equiv R / c_{p}\right]$

$\theta \quad$ potential temperature $\left[\equiv T\left(p_{s} / p\right)^{\kappa}\right]$

$X, Y$ mechanical forces

Equations 2.2 and 2.3 are the horizontal components of the momentum equation. Equation 2.4 is the thermodynamic energy equation, 2.5 is the continuity equation of mass, and 2.6 is the hydrostatic relation in the vertical. 


\subsection{Zonal Mean and Eddies}

The average circulation of the atmosphere features mean winds that are primarily zonal. They tend to blow roughly parallel to latitude circles. It is often convenient to separate the general atmospheric circulation into a longitudinally averaged (zonal mean) flow and departures from the mean flow. These departures are often known as waves or eddies. The mean flow is independent from longitude. In the troposphere, deviations from the zonal mean, which are generated by thermal forcing, topographical, and internal flow instabilities, exhibit a strong nonlinear character so that only idealized studies are possible with linear theory. On the other hand, eddies in the stratosphere and mesosphere primarily consist of planetary-scale waves, which can be accurately described in terms of linear wave dynamics. Therefore, the separation into the zonal mean and eddy components in the stratosphere and mesosphere provides a far more powerful tool for theoretical analysis compared to the troposphere. If $\chi$ is any variable that representing a physical quantity, its zonal-mean value $\bar{\chi}$ is defined as

$$
\bar{\chi}(\phi, z, t)=\frac{1}{2 \pi} \int_{0}^{2 \pi} \chi(\lambda, \phi, z, t) d \lambda
$$

This is often referred to as a Eulerian mean. Straightforward averages over a set of points fixed in space-time are known as Eulerian averages and are comparatively easy to perform with atmospheric data despite the theoretical advantages of Lagrangian averages that are taken over appropriately chosen set of moving fluid particles. To separate the zonal circulation and eddies, we define the motion of the eddies as the total motion minus the motion of the mean flow as follows.

$$
\chi^{\prime}(\lambda, \phi, z, t)=\chi(\lambda, \phi, z, t)-\bar{\chi}(\phi, z, t)
$$

If the departures from zonal symmetry are small, then the zonal mean state may 
be regarded as slowly varying in time. The amplitudes of the eddies are not always smaller than the amplitudes of the mean flow. For example, in the mid-latitude troposphere, the eddies are often of similar amplitude to the mean flow. Time evolution of small disturbances to the slowly varying zonally average flow can be described by linearizing the eddy equation. Hence, two classes of disturbances are evident in the primitive equations. These are sometimes referred to as Class I or Solutions of the First Kind and Class II or Solutions of the Second Kind. The first class consists of gravity wave modes and these waves tend to be apparent on small scales. The second class of waves allowed by the primitive equations consists of Rossby or planetary wave modes.

\subsection{Classical Tidal Theory}

This section is summarized from the "Tidal and Planetary Waves" tutorial by Forbes (1995). The primitive Equations 2.2 - 2.7 can be decomposed into a zonal mean term and a perturbation term by substitution of Equation 2.9. Once the sets of equations describing the zonal mean and perturbation are obtained, the equations can be decoupled by assuming the background atmosphere to be horizontally stratified and the zonal mean winds are zero. For the present mathematical analysis, assumption of an isothermal background atmosphere leads to further simplification of the perturbation equations. The linearized primitive equations governing small perturbations in a spherical isothermal atmosphere may be written as (Holton, 1975).

$$
\begin{gathered}
\frac{\partial u^{\prime}}{\partial t}-2 \Omega \sin \phi v^{\prime}+\frac{1}{a \cos \phi} \frac{\partial \Phi^{\prime}}{\partial \lambda}=0 \\
\frac{\partial v^{\prime}}{\partial t}+2 \Omega \sin \phi u^{\prime}+\frac{1}{a} \frac{\partial \Phi^{\prime}}{\partial \phi}=0
\end{gathered}
$$




$$
\begin{gathered}
\frac{\partial \Phi_{z}^{\prime}}{\partial t}+N^{2} w^{\prime}=\frac{\kappa J^{\prime}}{H} \\
\frac{1}{a \cos \phi}\left[\frac{\partial u^{\prime}}{\partial \lambda}+\frac{\partial\left(v^{\prime} \cos \phi\right)}{\partial \phi}\right]+\frac{1}{\rho_{o}} \frac{\partial\left(\rho_{o} w^{\prime}\right)}{\partial z}=0
\end{gathered}
$$

where $\left(u^{\prime}, v^{\prime}, w^{\prime}\right)$ are eastward, northward, and upward perturbation velocities, $\Phi^{\prime}$ is perturbation geopotential, $J$ is heating per unit mass.

Assume the perturbations to consist of longitudinally propagating waves of zonal wavenumber $s$ and frequency $\sigma$ :

$$
\left\{u^{\prime}, v^{\prime}, w^{\prime}, \Phi^{\prime}\right\}=\{\hat{u}, \hat{v}, \hat{w}, \hat{\Phi}\} \exp [i(s \lambda-\sigma t)]
$$

The zonal wavenumber is a positive integer $(s=0$ permitted $)$ that gives the number of maxima of the sinusoidal oscillation in longitudes. The $(s \lambda-\sigma t)$ form for the phase is chosen so that positive values for $\sigma$ correspond to eastward propagating waves and negative values to westward propagating waves. Substituting Equation 2.14 into Equations 2.10 - 2.13 and eliminating derivatives with respect to $t$ and $\lambda$, we get

$$
\begin{array}{r}
\hat{u}=\frac{f}{2 \Omega a\left(f^{2}-\mu^{2}\right)}\left[\frac{s}{\left(1-\mu^{2}\right)^{1 / 2}} \hat{\Phi}+\frac{\mu\left(1-\mu^{2}\right)^{1 / 2}}{f} \frac{\partial \hat{\Phi}}{\partial \mu}\right] \\
\hat{v}=\frac{-i f}{2 \Omega a\left(f^{2}-\mu^{2}\right)}\left[\frac{s \mu}{f\left(1-\mu^{2}\right)^{1 / 2}} \hat{\Phi}+\left(1-\mu^{2}\right)^{1 / 2} \frac{\partial \hat{\Phi}}{\partial \mu}\right] \\
\hat{w}=\frac{1}{N^{2}}\left[i 2 \Omega f \frac{\partial \hat{\Phi}}{\partial z}+\frac{\kappa \hat{J}}{H}\right] \\
\frac{i s \quad \hat{\mu}+\frac{1}{a} \frac{\partial}{\partial \mu}\left[\left(1-\mu^{2}\right)^{1 / 2} \hat{v}\right]+\frac{1}{\rho_{o}} \frac{\partial}{\partial z}\left(\rho_{o} \hat{w}\right)=0}{a\left(1-\mu^{2}\right)^{1 / 2}}
\end{array}
$$

where $f=\sigma / 2 \Omega$, and $\mu=\sin \phi$ 
Substituting Equations 2.15 - 2.17 into 2.18 can be consolidating into a single second-order partial differential equation for $\hat{\Phi}$ in $z$ and $\phi$ :

$$
\begin{aligned}
\frac{\partial}{\partial \mu}\left[\frac{\left(1-\mu^{2}\right)}{\left(f^{2}-\mu^{2}\right)} \frac{\partial \hat{\Phi}}{\partial \mu}\right] & -\frac{1}{\left(f^{2}-\mu^{2}\right)}\left[-\frac{s\left(f^{2}+\mu^{2}\right)}{f\left(f^{2}-\mu^{2}\right)}+\frac{s^{2}}{\left(1-\mu^{2}\right)}\right] \hat{\Phi} \\
& =\frac{4 \Omega^{2} a^{2}}{N^{2} \rho_{o}} \frac{\partial}{\partial z}\left(\rho_{o} \frac{\partial \hat{\Phi}}{\partial z}\right)-\frac{i 4 \Omega^{2} a^{2}}{\sigma N^{2} H \rho_{o}} \frac{\partial}{\partial z}\left(\rho_{o} \kappa \hat{J}\right)
\end{aligned}
$$

The Equation 2.19 may be solved by the method of separation of variables. Separable solutions of the following form exist

$$
\hat{\Phi}=\sum_{n} \Theta_{n}(\phi) G_{n}(z)
$$

where $\left\{\Theta_{n}\right\}$ is a complete orthogonal set referred as Hough functions and the vertical structure given by the vertical structure function $G_{n}(z)$. For example, since $\Theta_{n}$ is a complete orthogonal set, the thermal excitation can be expanded in the following form

$$
\hat{J}=\sum_{n} \Theta_{n}(\phi) J_{n}(z)
$$

Form Equations 2.10, 2.11, and 2.15, expressions for the horizontal velocity components in terms of $\Theta_{n}$ and $G_{n}$ may be derived

$$
\begin{aligned}
& \hat{u}=\frac{\sigma}{4 \Omega^{2} a} \sum_{n} U_{n}(\phi) G_{n}(z) \\
& \hat{v}=\frac{-i \sigma}{4 \Omega^{2} a} \sum_{n} V_{n}(\phi) G_{n}(z)
\end{aligned}
$$

where

$$
U_{n}=\frac{1}{\left(f^{2}-\sin ^{2} \phi\right)}\left[\frac{s}{\cos \phi}+\frac{\sin \phi}{f} \frac{d}{d \phi}\right] \Theta_{n}
$$




$$
V_{n}=\frac{1}{\left(f^{2}-\sin ^{2} \phi\right)}\left[\frac{s \tan \phi}{f}+\frac{d}{d \phi}\right] \Theta_{n}
$$

The following expression is a consequence of separation:

$$
i \sigma H\left[\frac{1}{\rho_{o}} \frac{\partial}{\partial z} \rho_{o} \frac{\partial}{\partial z} G_{n}\right]+\frac{1}{\rho_{o}} \frac{\partial}{\partial z}\left(\rho_{o} \kappa J_{n}\right)=-\frac{i \sigma \kappa}{h_{n}} G_{n}
$$

where $h_{n}$ arises as the separation constant. This result can be simplified by defining $G_{n}^{\prime}=G_{n} \rho_{o}^{1 / 2} N^{-1}$, taking $N^{2}=\kappa g / H$ for an isothermal atmosphere where $H=$ constant $=7.5 \mathrm{~km}$ (corresponding to $T_{0}=256 \mathrm{~K}$ ). Letting $x=z / H$, results in the canonical form for the vertical structure equation (for an isothermal atmosphere):

$$
\frac{d^{2} G_{n}^{\prime}}{d x^{2}}+\left[\frac{\kappa H}{h_{n}}-\frac{1}{4}\right] G_{n}^{\prime}=-\frac{\rho_{o}^{-1 / 2}}{i \sigma N} \frac{d}{d x}\left(\rho_{o} J_{n}\right)
$$

The $\phi$ - dependent part of the solution (meridional structure) is embodied in Laplace's Tidal Equation (Chapman and Lindzen, 1970).

$$
\frac{d}{d \mu}\left[\frac{\left(1-\mu^{2}\right)}{\left(f^{2}-\mu^{2}\right)} \frac{d \Theta_{n}}{d \mu}\right]-\frac{1}{\left(f^{2}-\mu^{2}\right)}\left[-\frac{s}{f} \frac{\left(f^{2}+\mu^{2}\right)}{\left(f^{2}-\mu^{2}\right)}+\frac{s^{2}}{\left(1-\mu^{2}\right)}\right] \Theta_{n}+\epsilon_{n} \Theta_{n}=0
$$

where $\epsilon_{n}=(2 \Omega a)^{2} / g h_{n}$. Thus, the equation governing atmospheric perturbations are now formulated in terms of an eigenfunction-eigenvalue problem. Note that Equations 2.27 and 2.28 are linked through $h_{n}$, the set of eigenvalues, which is referred to as the "equivalent depths." This nomenclature originates from the first appearance of Equation 2.28 in connection with the ocean tide problem where $\mathrm{h}$ is the ocean depth (Laplace, 1799; Taylor, 1936). 


\subsubsection{Vertical Structure Equation: Forced and Free Solutions}

With given suitable boundary conditions, Equation 2.27 has a unique solution for the vertical structure. Rewrite Equation 2.27 as follows

$$
\frac{d^{2} G_{n}^{\prime}}{d x^{2}}+\alpha^{2} G_{n}^{\prime}=F(x)
$$

where $\alpha^{2}=\kappa H / h_{n}-1 / 4$, the form of the solution to Equation 2.29 is

$$
G_{n}^{\prime} \sim A e^{i \alpha x}+B e^{-i \alpha x}
$$

When $F(x) \neq 0$ ("forced" solution), there are two possibilities. If $h_{n}<0$ or $h_{n}>4 \kappa H$, then $\alpha^{2}<0$ and

$$
G_{n}^{\prime} \sim e^{-|\alpha| x}
$$

above the source region for a bounded solution. In this case, the solutions are referred to as "evanescent" or "trapped" since the wave oscillations are more or less confined to the region of excitation. If $0<h_{n}<4 \kappa H$, then $\alpha^{2}>0$ and a "radiation condition" at $x=\infty$ implies

$$
G_{n}^{\prime} \sim e^{i \alpha x}
$$

where the plus (minus) sign in the expression for $\alpha= \pm\left(\kappa H / h_{n}-1 / 4\right)^{1 / 2}$ is chosen for westward (eastward) propagating waves. This is the so-called propagating solution, where the wave propagates away from the source region.

"Free" solution $(F(x)=0)$ is the only nontrivial solution remaining bounded and satisfying $w=0$ at $z=0$ is

$$
G_{n}^{\prime} \sim e^{\left(\kappa-\frac{1}{2}\right) x}
$$


and

$$
h_{n}=\frac{H}{1-\kappa}
$$

where $h_{n}=10.5 \mathrm{~km}$ for $H=7.5 \mathrm{~km}$. This free solution is corresponds to the resonant response of the atmosphere. These kinds of waves are known as "Lamb" or "edge" waves. They exhibit exponential energy decay with altitude while horizontal velocity and other wave fields increase exponentially by a factor of 40 from the surface to $100 \mathrm{~km}$. Without dissipation, such free oscillations would continue indefinitely without forcing.

Although the global forms of the tidal structure are quite complicated, some general features can be deduced from the basic properties of the vertical structure equation. If $h_{n}<0$, or $h_{n}=\infty$, these modes are trapped in the vertical near the forcing region. If $0<h_{n}<4 \kappa H \approx 8 H / 7$, a tidal mode is roughly sinusoidal in the vertical, with the vertical wavelength defined by

$$
\lambda_{n}=\frac{2 \pi H}{\sqrt{\frac{\kappa H}{h_{n}}-\frac{1}{4}}}
$$

The vertical wavelength provides a more physically meaningful vertical scale value than equivalent depth.

\subsubsection{Laplace's Tidal Equation}

Laplace's tidal equation is often written as follows to emphasize the explicit dependences on $s, \sigma$, and $\epsilon_{n}$

$$
F\left(\Theta_{n}^{s, \sigma}\right)+\epsilon_{n}^{s, \sigma} \Theta_{n}^{s, \sigma}=0
$$


where

$$
F=\frac{d}{d \mu}\left[\frac{\left(1-\mu^{2}\right)}{\left(f^{2}-\mu^{2}\right)} \frac{d}{d \mu}\right]-\frac{1}{f^{2}-\mu^{2}}\left[-\frac{s\left(f^{2}+\mu^{2}\right)}{f\left(f^{2}-\mu^{2}\right)}+\frac{s^{2}}{\left(1-\mu^{2}\right)}\right]
$$

For each choice of $s$ and $\sigma$ there exist sets of $\epsilon_{n}$ and $\Theta_{n}$ which satisfies the Equation 2.37 and the boundary conditions at the poles. The collection of all $\Theta_{n}$ are the eigenfunctions of Laplace's tidal equation and are called Hough functions. Hough functions $\Theta_{n}^{s, \sigma}$ are orthogonal and may be decomposed as a series of associated Legendre polynomials with $|n| \geq s$.

$$
\Theta_{n}^{s, \sigma}=\sum_{m=s}^{\infty} C_{n, m}^{s, \sigma} P_{m}^{s}(\mu)
$$

where $m$ is the order of the associated Legendre functions and $C_{n, m}^{s, \sigma}$ is the expansion coefficients. $s$ is the zonal wavenumber and $n$ is the meridional index that provides information on the number of latitudinal nodes and symmetry characteristics. It is common to refer particular mode as the $\Theta_{n}^{s}$ mode or just the $(s, n)$ mode.

The mathematical and physical theories described above are highly complicated and can only approximate the real atmosphere from first principles. Tidal motions in the lower and middle atmosphere are reasonably able to be explained by the theories above, under the assumptions of no background winds, no dissipative forces, and no nonlinear interactions. However, this method (classical tidal theory) does not work when vertical diffusion of heat and momentum in a spherical rotating sphere, or latitude dependent mean winds in a diffusive or non-diffusive atmosphere are included, because mathematical solutions become inseparable in the latitudinal and vertical coordinates. This necessitates a numerical approach to the problem wherein a universal set of modal structures does not naturally emerge. Review about these numerical models can be found in Walterscheid and Venkateswaran (1979), Lindzen and Hong (1974), Forbes and Garrett (1979), and Forbes (1982a,b). Dissipation processes such 
as Newtonian cooling, molecular viscosity, ion drag, and thermal conductivity are important because they serve to damp the tides, particularly in the MLT region. Nonlinear terms in the momentum equations, wave forcing mechanisms such as solar absorption, latent heat release, and localized excitation sources are also necessary. General circulation models (GCM) such as the eCMAM (extended Canadian Middle Atmospheric Model), which is the GCM used in this dissertation, does not make any simplifications to the primitive equations and have implemented many of these real atmospheric processes, hence making it a very powerful tool.

Amplitudes of the upward propagating tides are increasing with altitude as the density decreases and the may dissipate and deposit their energy and momentum in the middle and upper atmosphere. Tidal damping in the atmosphere can come from a variety of sources, including radiative damping, molecular diffusion, eddy diffusion, and eddy flux divergence through interaction of tides with gravity waves (Ortland, 2005). The predominant dissipative force in the MLT region is eddy dissipation generated by the breaking of upwards propagating gravity waves, especially in the region below the turbopause. When breaking, gravity waves deposit momentum in to the MLT region and cause drag or stress in the background wind fields. Forcing from breaking gravity waves enhances or attenuates the tide, thus resulting in an increase or decrease in tidal vertical wavelength. Above the turbopause, molecular diffusion becomes dominant as the mean free path for particles increases.

\subsection{Momentum and Thermodynamic Budget Equations}

The primitive equations for zonal momentum and thermodynamic equations (Equation 2.2 and 2.4) describing the atmosphere in eCMAM general circulation model 
may express as the following form

$$
\begin{aligned}
& \frac{\partial u}{\partial t}= f v-\frac{1}{a \cos \phi} \frac{\partial \Phi}{\partial \lambda}-\vec{v} \cdot \nabla u+u v \frac{\tan \phi}{a} \\
&+F_{G W D, x}+F_{D i f f, x}+F_{\text {ion }, x}+F_{\text {other } x} \\
& \frac{\partial T}{\partial t}=-\vec{v} \cdot \nabla T-\frac{w R T}{c_{p} p}+Q_{\text {short }}+Q_{\text {long }}+Q_{\text {chemical }} \\
&+Q_{\text {convectives }}+Q_{\text {Diff }}+Q_{\text {other }}
\end{aligned}
$$

The advection terms in the zonal momentum Equation 2.39 can be written as follows

$$
F_{\text {advec }, u}=\vec{v} \cdot \nabla u=-\left(\frac{u}{a \cos \phi} \frac{\partial u}{\partial \lambda}+\frac{v}{a} \frac{\partial u}{\partial \phi}+w \frac{\partial u}{\partial z}\right)
$$

Advection terms in the thermodynamic Equation 2.40 can be written as follows

$$
F_{\text {advec }, T}=\vec{v} \cdot \nabla T=-\left(\frac{u}{a \cos \phi} \frac{\partial T}{\partial \lambda}+\frac{v}{a} \frac{\partial T}{\partial \phi}+w \frac{\partial T}{\partial z}\right)
$$

where $u, v, w, T$, and $\Phi$ are zonal wind, meridional wind, vertical wind velocity, temperature, and geopotential, respectively. The mean radius of Earth is designated by $a$. The variable $f=2 \Omega \sin \phi$ is the Coriolis parameter. $\phi$ and $\lambda$ are latitude and longitude, respectively. $R$ is dry air gas constant, and $C_{p}$ is the specific heat at constant pressure. The left-hand-side (LHS) of Equation 2.39 is zonal wind tendency (acceleration). Terms on the right-hand-side (RHS) of Equation 2.39 are the Coriolis force $(\mathrm{CF})$, pressure gradient force (PGF), advection, curvature, and the remaining terms are model physics that comes from various parameterization (eddy diffusion, ion drag, and gravity wave drag) schemes in the model. $F_{G W D}$ is the sum of nonorographic and orographic gravity wave drag (GWD), $F_{\text {diff }}$ is vertical eddy and molecular diffusion, $F_{\text {ion }}$ is ion drag and $F_{\text {other }}$ is all other forcing terms. $F_{\text {other }}$ can be obtained from the difference between the LHS and RHS terms. The differences arise 
from different calculation methods used in the model and here, the effect of nudging that is not implicitly included in the previous forcing terms, etc. In classical tidal theory, only the first two terms on the RHS, the CF and PGF are used to determine the time tendencies of the horizontal tidal winds, thus referred as the classical terms (Chapman and Lindzen, 1970; Lu et al., 2012; McLandress, 2002a). All the other terms are non-classical terms. The LHS of Equation 2.40 is the temperature tendency (heating rate). Thermodynamic budget terms on the RHS are divided into three categories: advection (first term), adiabatic heating (second term), and total diabatic heating (the remainder six terms). $Q_{\text {short }}$ and $Q_{\text {long }}$ are short wave heating and long wave cooling. Respectively, $Q_{\text {chemical }}$ is chemical heating, and $Q_{\text {convective }}$ is the heating due to convective adjustment, $Q_{\text {diff }}$ is the heating due to vertical diffusion, and $Q_{o t h e r}$ is all other terms and includes such processes as eddy vertical diffusion, GW heating, and conversion of kinetic energy into heat resulting from the dissipation of horizontal winds (Fomichev et al., 2002).

The procedure to calculate the LHS term and RHS terms in Equations 2.39 and 2.40 for migrating diurnal tide DW1 can be separated into three steps.

Step 1: calculate the LHS term

To obtain the DW1 amplitude and phase, the 2D fast Fourier transform (FFT) is applied to the temperature, zonal wind, or meridional wind output from the model. Here we use Equation 2.40 as an example. The tidal oscillations in temperature can be defined as

$$
T^{\prime}=\hat{T} \exp [-i(\sigma t+s \lambda)]=A e^{i p} e^{-i(\sigma t+s \lambda)}
$$

where $\hat{T}$ is complex amplitude of temperature where $\hat{T}=A e^{i p}, A$ is tidal amplitude in temperature and $p$ is tidal phase. The absolute value of the LHS can be written as $L H S=\left|\partial T^{\prime} / \partial t\right|=\sigma \cdot A$, where $\sigma$ is tidal frequency $(\sigma=2 \pi$ for DW1) and $s$ is zonal wave number ( $s=1$ for DW1). The LHS of Equation 2.40 becomes 
tidal amplitude in temperature multiply a constant $2 \pi$ for DW1. This procedure can also use for the other tides as well.

Step 2: calculate the RHS terms

RHS terms of the Equation 2.40 such as the advection and adiabatic terms can be calculated directly using the wind and temperature outputs from the model. The parameterized heating terms are direct output from the model. Tidal components for each heating terms in RHS can be obtained by applying 2D FFT on the RHS terms. By analyzing the various terms on the RHS of the equation, we should gain some insights into the causes of the tidal amplitude variations on the LHS.

Step 3: separate the advection term into linear and nonlinear terms

The Equation 2.42 can be further separated into a linear advection term (wavemean interaction) and a nonlinear advection term (wave-wave interaction). Substituting the linearization expressions, Equation 2.9 into Equation 2.42 and similar to that in Lu et al. (2012), the linear advection term in the thermodynamic equation can be written as follows

$$
F_{\text {linearadvec }, T}=-\frac{\bar{u}}{a \cos \phi} \frac{\partial T^{\prime}}{\partial \lambda}-\frac{\bar{v}}{a} \frac{\partial T^{\prime}}{\partial \phi}-\frac{v^{\prime}}{a} \frac{\partial \bar{T}}{\partial \phi}-\bar{w} \frac{\partial T^{\prime}}{\partial z}-w^{\prime} \frac{\partial \bar{T}}{\partial z}
$$

The complex amplitude of the nonlinear advection term can then be obtained by subtracting the complex amplitude of the linear advection term from the complex amplitude of the total advection term. Similarly, the advection term in the momentum budget equation can also divided into linear and nonlinear advection term.

When different terms are combined, complex amplitudes are used so both amplitude and phase are taken into account. For example: the RHS is the sum of the complex amplitudes of all terms on the RHS of the equations except $Q_{\text {other }}$ in the Equation 2.40. The advection term is the sum of the complex amplitudes of nonlinear and linear advection terms. 


\section{CHAPTER 3}

\section{THE ECMAM AND SABER DATA}

This chapter provides a summary of the eCMAM30 and SABER data that are used to conduct the research in this dissertation. The primary goals of this dissertation are to make significant progress in our current understanding of the short-term tidal variability and its characteristics by performing statistical analysis and studying the underlying physical mechanisms. To achieve these, as an initial step, results from eCMAM30 are compared to those from the SABER observations. A comparison between the results from the model and observations will be helpful in assessing our ability to simulate the main features in tidal variability and gain confidence in the subsequent physical mechanism analysis.

\subsection{The eCMAM}

The eCMAM is a very sophisticated and three-dimensional nonlinear general circulation model which is based on the standard version of the CMAM. It is a research

project funded by the Canadian Space Agency (CSA) to provide an estimate of the chemical and dynamical evolution of the atmosphere over the period of 19792010. Detailed model descriptions of the standard CMAM and the third generation CCCMA-GCM (Canadian Centre for Climate Modelling and Analysis - General Circulation Model) from which the standard CMAM was built can be found in Beagley et al. (1997) and Scinocca et al. (2008), respectively. 
To develop the extended version of the CMAM, the upper boundary has been raised from $6 \times 10^{-7} \mathrm{mbar}$ (geopotential height $\sim 95 \mathrm{~km}$ ) to $2 \times 10^{-7}$ mbar (geopotential height $\sim 210 \mathrm{~km}$ ), providing a dissipation region for upward propagating waves via molecular diffusion and ion drag. The eCMAM is nonlinear and capable of integrating the full set of primitive equations (see Equations $2.2-2.7$ ) to resolve the global variation of the atmosphere as a function of time. The governing equations are solved in spectral space, with dependent variables expanded horizontally in spherical harmonics through triangular spectral truncation at wave number 32 (T32). This corresponds to a horizontal grid of $\sim 6^{\circ} \times 6^{\circ}$ latitudinal-longitudinal resolution. There are 87 vertical levels extending from the ground with a resolution varying from $\sim 150 \mathrm{~m}$ near the surface to $\sim 2 \mathrm{~km}$ near the tropopause and $\sim 3 \mathrm{~km}$ in the middle atmosphere. Tidal oscillations are self-consistently generated from short and long wave radiation absorption, large-scale condensation, and convective heating. Apart from the physical parameterizations that are inherited from the standard CMAM, the eCMAM improves the simulations by adding new physical parameterizations relevant to the mesosphere and thermosphere. The model includes physical processes such as a nonlocal thermal equilibrium (LTE) parameterization for the $15 \mu \mathrm{m} \mathrm{CO}_{2}$ band, solar heating due to absorption by $\mathrm{O}_{2}$ in the Schumann-Runge bands and by $\mathrm{N}_{2}, \mathrm{O}_{2}$ and $\mathrm{O}$ in the extreme ultra-violet (EUV) spectral region, parameterized chemical heating, molecular diffusion and viscosity, ion drag, modified non-orographic gravity wave drag (GWD). The detailed information on these parameterization schemes used in the eCMAM is referred to Fomichev et al. (2002) and references therein.

It has a comprehensive interactive neutral chemistry applied above $400 \mathrm{mbar}$ (de Grandpré et al., 2000) and a simplified interactive ion chemistry scheme (Beagley et al., 2010; Shepherd et al., 2014) applied above approximately $80 \mathrm{~km}$. These chemistry schemes altogether include 49 neutral species, electrons and 5 ions and employ 102 neutral, 27 ion, and 49 photolysis reactions. Approximately 99\% of the neutral 
atmosphere is actively simulated, the remaining $1 \%$ being noble gases. The model does not include ionospheric processes such as geomagnetic effects.

As shown in Beagley et al. (2000), the eCMAM is capable of producing realistic diurnal tides and semidiurnal tides below $120 \mathrm{~km}$, including the semiannual variation of the propagating diurnal tide in the mesosphere and it is in good agreement with WINDII and HRDI observations of mean winds and tides. UARS observations of the zonal-mean wind in the MLT region are in good agreement with the simulations from eCMAM (Fomichev et al., 2002). Du (2008) validated the altitudinal-latitudinal structures and seasonal variations of the eCMAM-generated diurnal and semidiurnal tidal winds with the observations. The 7 nonmigrating diurnal components and 4 nonmigrating semidiurnal components from the model are compared to the corresponding TIDI wind observations. The basic latitudinal structures for the amplitudes agree well between CMAM and TIDI, although the TIDI amplitudes is about 50\% smaller than the CMAM results for the DE3 zonal component, the DE2 zonal and meridional components, the DE1 zonal and meridional components. Comparison of diurnal tides and ground based observations in Chang et al. (2012) shown that the latitudinal structure of the diurnal tide wind fields resolved by the eCMAM is consistent with the ground-based observations. Many other studies have also shown that the eCMAM can simulate realistic tidal oscillations in the stratosphere and MLT region (Beagley et al., 2000; Chang et al., 2012; Du et al., 2007, 2014; Du and Ward, 2010; Zeng et al., 2008). The latitude-altitude structure and seasonal variation of DW1 in the thermosphere from the eCMAM30 compares very well with the TIE-GCM (Jones et al., 2017; Siskind et al., 2014), the GSWM (Hagan et al., 2001) and the WACCM-X (Liu et al., 2010a).

More recently, temperatures and winds below $10 \mathrm{hPa}$ (approximately $35 \mathrm{~km}$ ) are nudged (i.e., relaxed) to the 6 hourly temperature and horizontal winds from the European Center for Medium-Range Weather Forecasts (ECMWF) Interim Reanalysis 
(ERA-Interim) data from January 1979 to June 2010 (hence eCMAM30). Between 10 and $1 \mathrm{hPa}$ (approximately $50 \mathrm{~km}$ ) the amount of nudging is linearly reduced to zero, so that above $1 \mathrm{hPa}$ the model is free running. Reanalysis variables at intermediate times between adjacent $6 \mathrm{~h}$ intervals are computed using linear interpolation. The nudging approach is used to constrain primarily the synoptic space and time scales, which are well represented in the reanalysis data. Relaxation is applied to horizontal scales in spectral space with $n \leq 21$, where $n$ is the total wave number. The form of the nudging tendency is $-\left(X-X_{R}\right) / \tau_{0}$, where $\tau_{0}=24$ hours is the relaxational time scale and $X$ and $X_{R}$ are the model and reanalysis spectral vorticity, divergence or temperature coefficients (Dee et al., 2011; Kharin and Scinocca, 2012; McLandress et al., 2013, 2014).

Nudging to the reanalysis data in the lower atmosphere allows realistic interaction between the atmospheric waves and the background atmosphere and thus includes realistic quasi-biennial oscillation $(\mathrm{QBO})$ in the stratosphere. The sea surface temperature (SST) in the eCMAM30 uses monthly global-complete fields of SST and sea ice concentration at each latitude-longitude grid from the Met Office Hadley Center's sea ice and sea surface temperature data set (HadISST) during the same time period of 1979-2010. Therefore, El Niño-Southern Oscillation (ENSO) is also included in the model in a realistic way. The solar irradiance scheme in the model takes into account the day-to-day variations of the spectral solar irradiance (SSI) over the full solar cycle (Forster et al., 2011; Semeniuk et al., 2011). SSI is used to calculate heating below $\sim 120 \mathrm{~km}$ whereas F10.7 solar flux is used for ionization rates EUV heating above $\sim 120 \mathrm{~km}$. The spectral solar irradiance is specified using the data from SOLARISHEPPA website (https://solarisheppa.geomar.de/) and the F10.7 cm index is taken from the International Reference Ionosphere (IRI-2007) model package (Bilitza and Reinisch, 2008). Medium geomagnetic forcing is set as a constant in the ion drag scheme. The nudged eCMAM30 is capable of realistically reproducing the cli- 
matology of the background temperature, migrating and nonmigrating diurnal tidal components when compared to SABER observations (Gan et al., 2014).

The model outputs (zonal wind, meridional wind, temperature and geopotential height, etc.) are extracted from the model every $6 \mathrm{~h}$ interval for the period of January 1979 to June 2010. In order to derive the tides from eCMAM output, a two-dimensional Fourier transform in longitude and time can be applied to the global temperature to get the spectrum density in the domain of wave frequency and zonal wavenumber. An inverse Fourier transformation gives the amplitude and phase of a particular atmospheric tide with specified wavenumber and frequency. Figure 3.1 shows the normalized mean amplitude of diurnal tidal temperature components from wavenumber -5 to +5 at $100 \mathrm{~km}$ as a function of latitude for March 2008 .

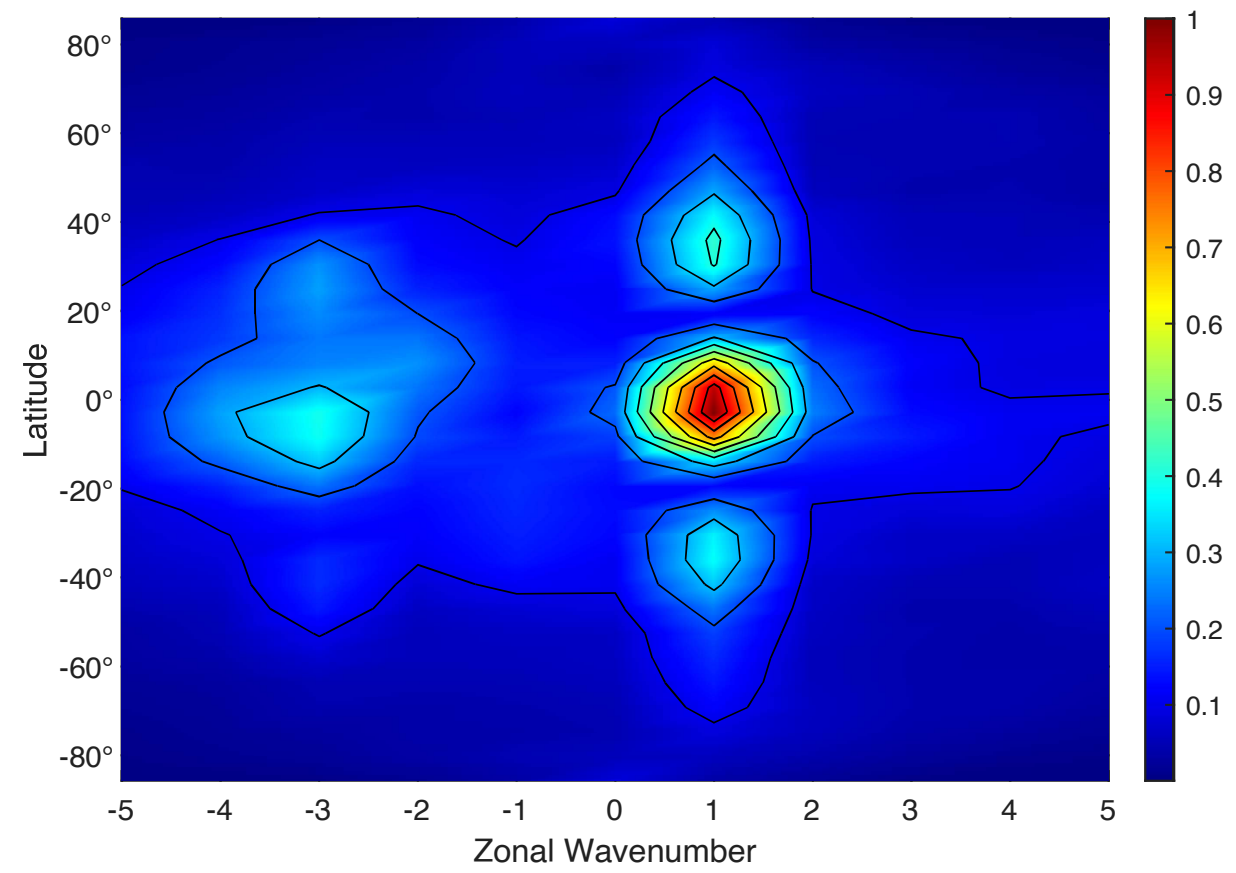

Figure 3.1. Normalized wavenumber spectra for diurnal tides T (K) at $100 \mathrm{~km}$ as a function of latitude from the eCMAM in March 2008.

The amplitude is normalized by dividing the maximum amplitude at the same altitude $(100 \mathrm{~km})$. Positive (negative) wavenumber denotes westward (eastward) propagation tidal component. Relatively, DW1 tidal component is dominant out of 11 
tidal components in the spectrum. DW1 also shows two secondary maxima around midlatitudes in each hemisphere at $100 \mathrm{~km}$. In addition to DW1, DE3 is present in the range of $30^{\circ} \mathrm{S}-30^{\circ} \mathrm{N}$ with $\sim 30-40 \%$ of the maximum amplitude of DW1.

Schematic cross section of the seasonally averaged zonal mean background temperature in Northern Hemisphere spring (March, April, May), summer (June, July, and August), autumn (September, October, and November), and winter (December, January, and February) obtained from the eCMAM30 between 1979-2010 are shown in Figure 3.2. The model can reproduce the latitudinal temperature gradients quite well (Gan et al., 2014). There is a year-round cold mesopause with minimum temperature and warm stratopause with maximum temperature at the summer solstice. Temperature increases rapidly in the thermosphere. Comparison of the background temperature from the eCMAM30 with SABER data for different seasons shows that both data sets are in good agreement throughout the vertical domain between $20 \mathrm{~km}$ to $120 \mathrm{~km}$ (Gan et al., 2014).

Figure 3.3 shows the climatological latitude-altitude cross sections of zonal wind from eCMAM30 over a 12 month timespan. Some common features are well reproduced by the model. The upper tropospheric jet streams in both hemispheres are strong and eastward throughout the year. The stratosphere and lower mesosphere are characterized by winter eastward and summer westward wind in each hemisphere. Figure 3.4 shows the climatological latitude-altitude cross sections of meridional wind from eCMAM30 over the annual cycle. Compared to the zonal mean wind, the meridional wind is much weaker. Below $100 \mathrm{~km}$, Meridional wind is characterized by northward flow around winter solstice and southward flow around summer solstice. Both flows are primarily driven by the non-orographic gravity wave drag (McLandress et al., 2006). 

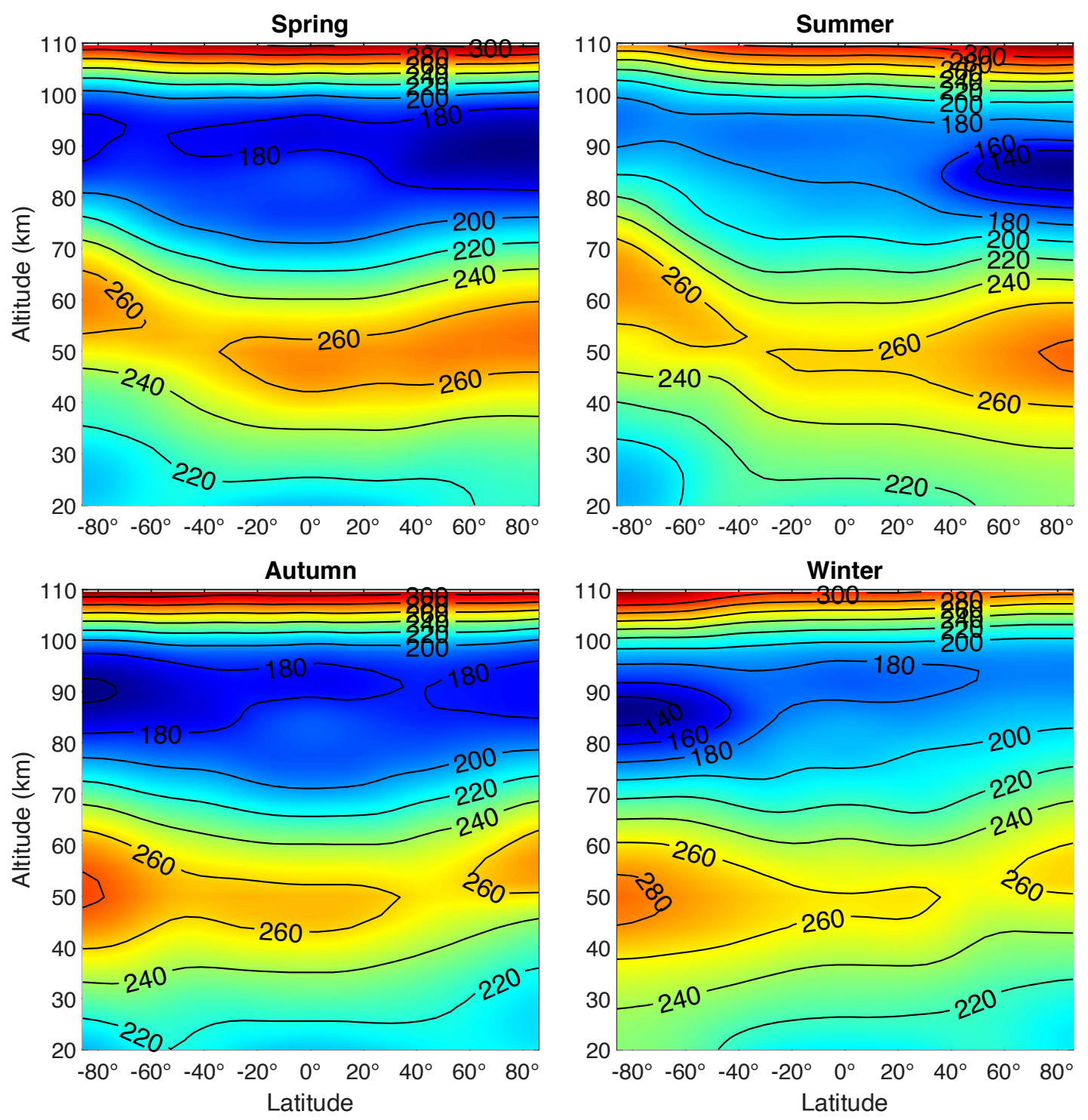

Figure 3.2. Climatology of zonal and seasonal mean background temperature (K) from the eCMAM30 as a function of latitude and altitude. 

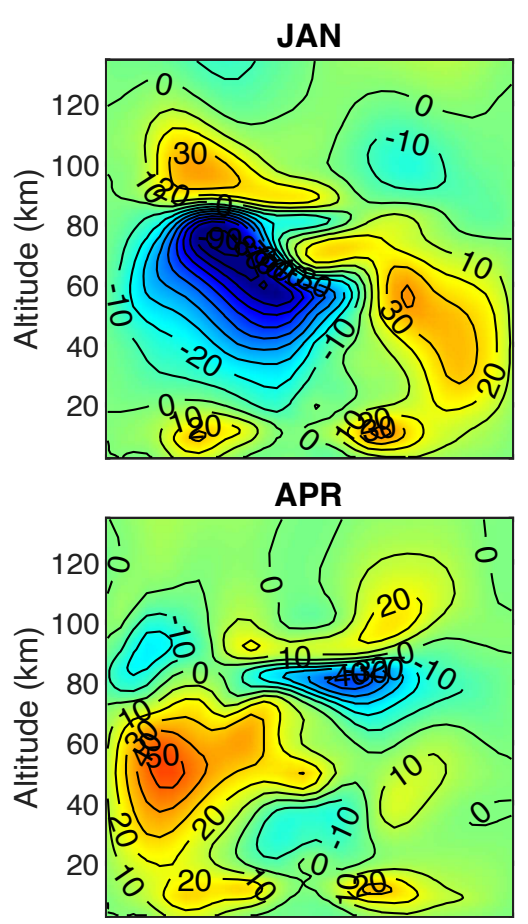

JUL
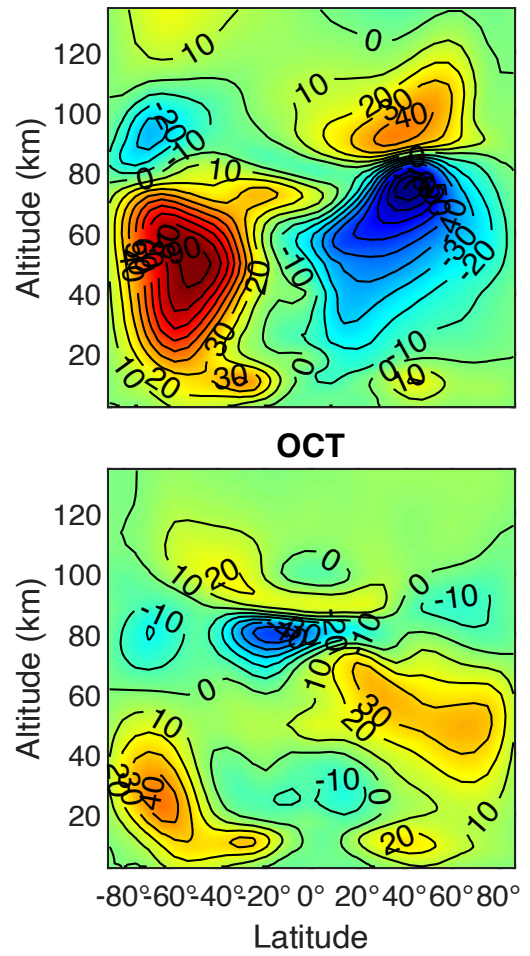

Background U (m/s)

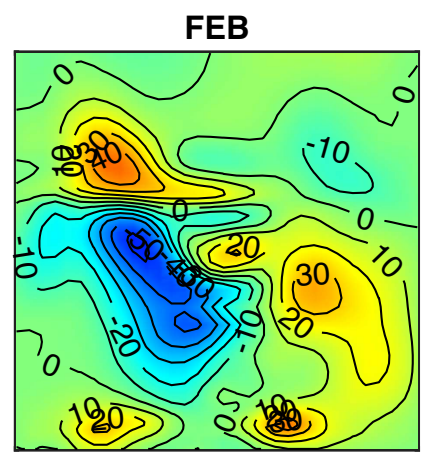

MAY

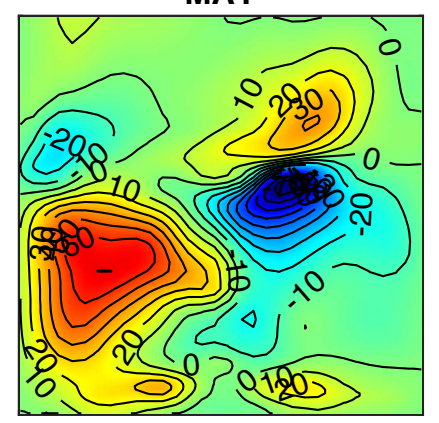

AUG
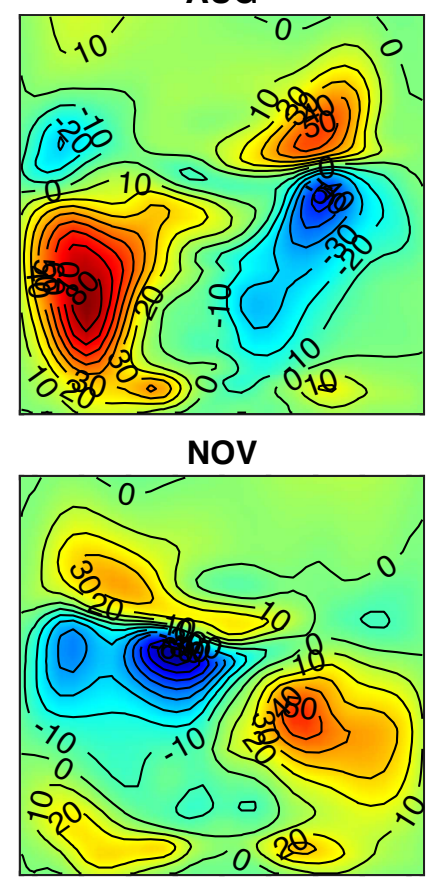

$-80^{\circ} 60^{\circ}-40^{\circ}-20^{\circ} 0^{\circ} 20^{\circ} 40^{\circ} 60^{\circ} 80^{\circ}$ Latitude
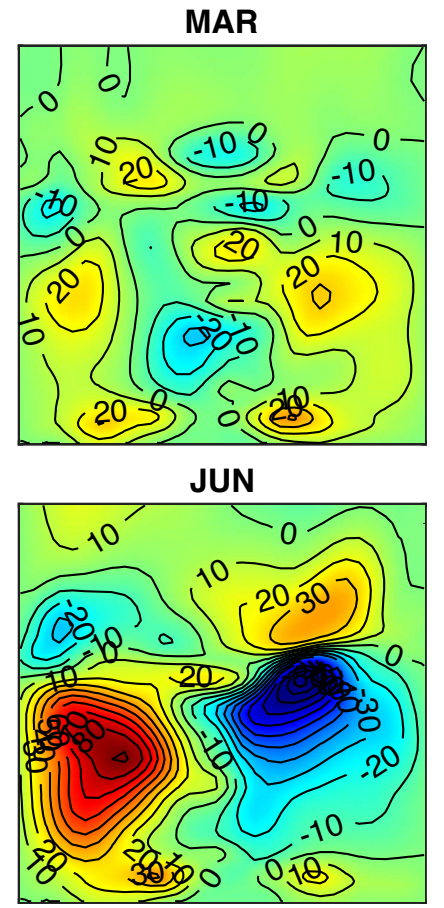

SEP

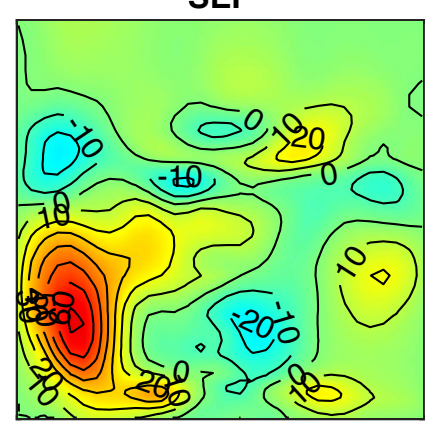

DEC

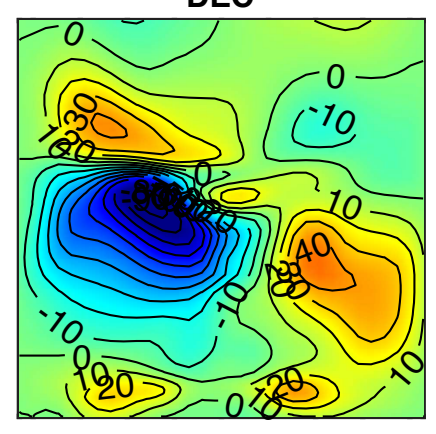

$-80^{\circ} 60^{\circ}-40^{\circ}-20^{\circ} 0^{\circ} 20^{\circ} 40^{\circ} 60^{\circ} 80^{\circ}$

Latitude

Figure 3.3. Climatological mean of zonal wind $\left(\mathrm{m} \mathrm{s}^{-1}\right)$ from eCMAM 30 as a function of latitude and altitude. Blue-colored (negative) regions are westward and the redcolored (positive) regions are eastward. 


\section{Background V (m/s)}
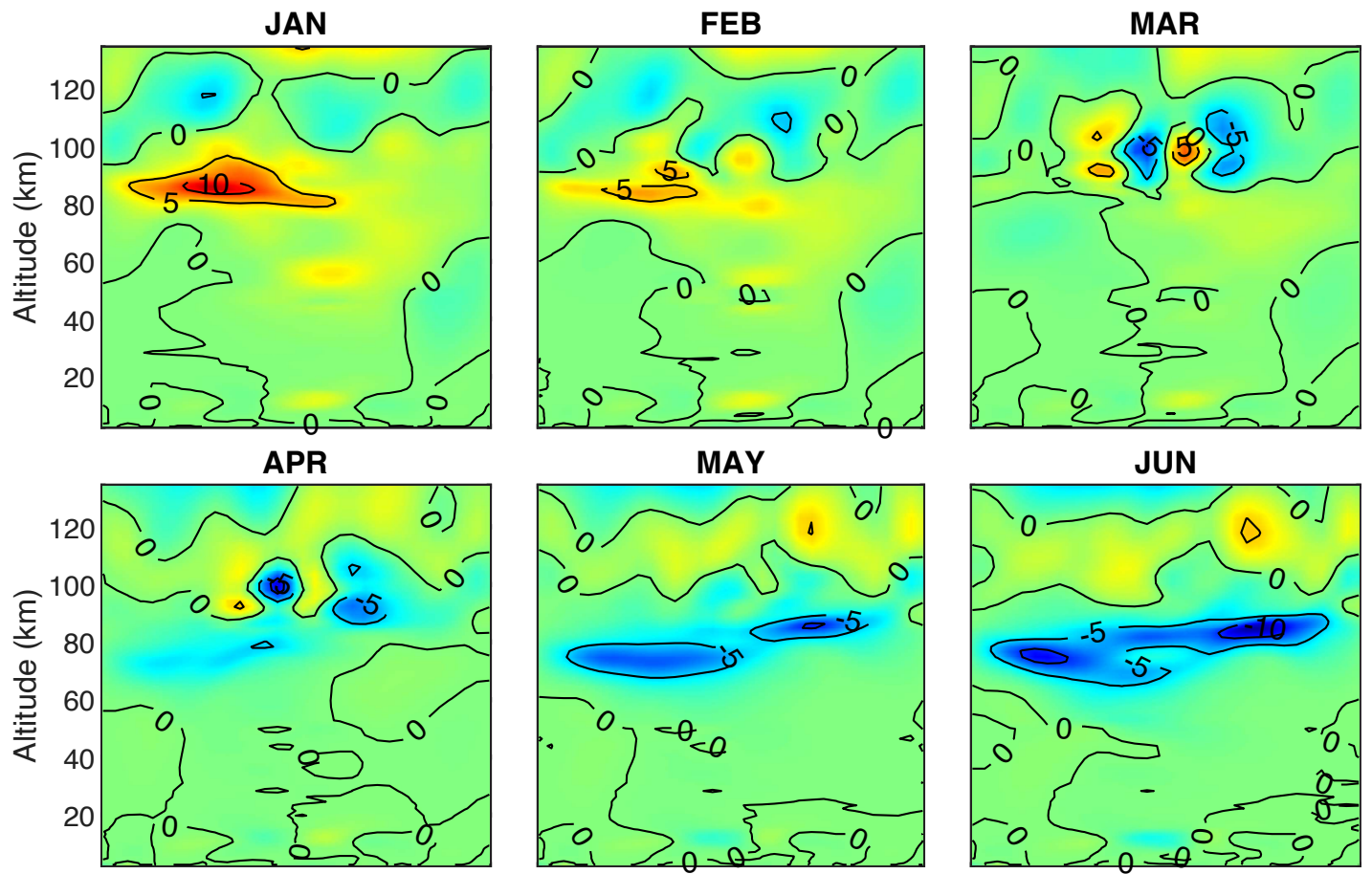

MAY

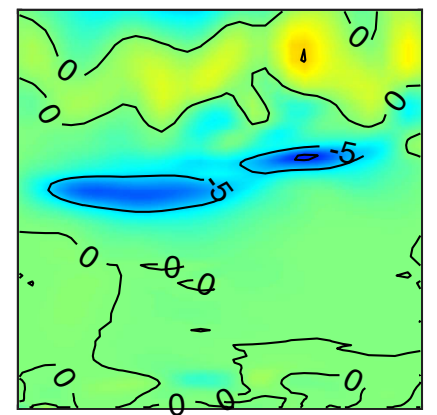

JUN

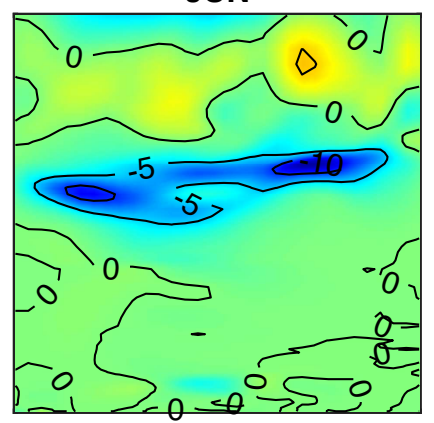

JUL

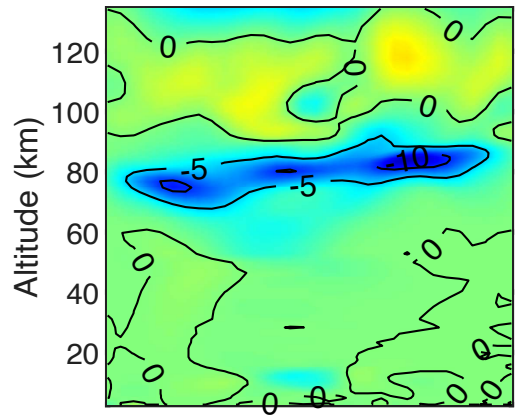

AUG

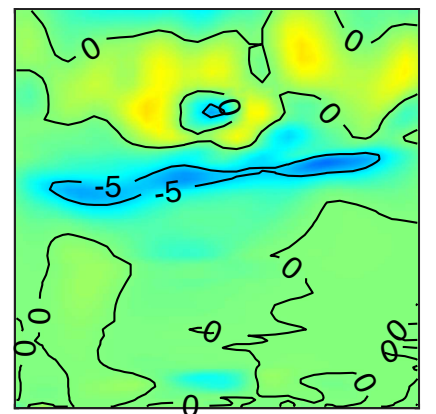

SEP

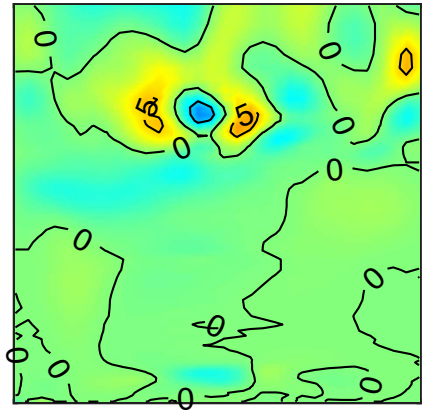

OCT
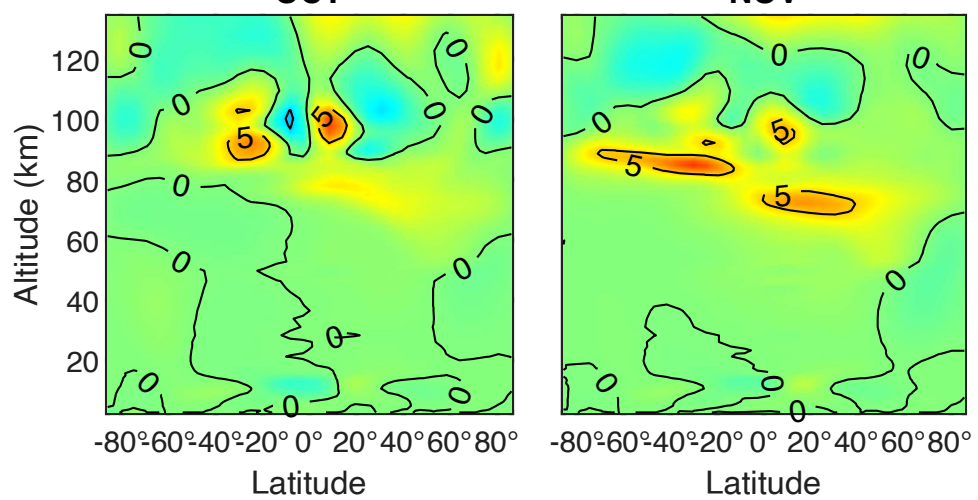

DEC

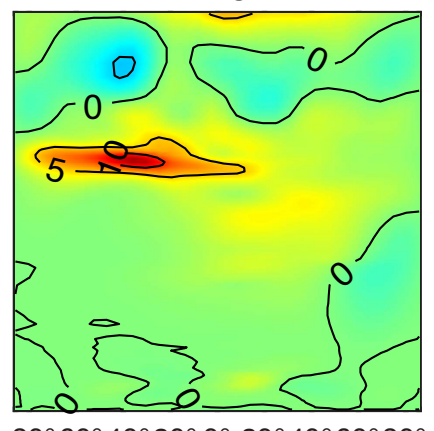

$-80^{\circ}-60^{\circ}-40^{\circ}-20^{\circ} 0^{\circ} 20^{\circ} 40^{\circ} 60^{\circ} 80^{\circ}$ Latitude

Figure 3.4. Climatological mean of meridional wind $\left(\mathrm{m} \mathrm{s}^{-1}\right)$ from eCMAM30 as a function of latitude and altitude. Blue-colored (negative) regions are southward and the red-colored (positive) regions are northward. 
The primary tide studied in this dissertation is the migrating diurnal tide DW1 ( $\mathrm{Gu}$ and $\mathrm{Du}, 2018$ ). The eCMAM can simulate a realistic migrating diurnal tide, including the semiannual variation of the amplitude of the propagating diurnal tide in the mesosphere (McLandress, 2002a,b). Figure 3.5 shows the latitude-height crosssections of DW1 amplitudes for temperature $\mathrm{T}(\mathrm{K})$ and phases (in hour) in the stratosphere $(10-50 \mathrm{~km})$ and mesosphere $(50-100 \mathrm{~km})$ for March. The amplitudes show four distinct latitudinal structures with height. DW1 T amplitude $(\sim 0.5 \mathrm{~K})$ maximizes over the equator in the stratosphere below $35 \mathrm{~km}$. The latitudinal structure changes above $35 \mathrm{~km}$ and DW1 T maximizes in the mid latitudes $\left(30^{\circ}-50^{\circ}\right)$ of both hemispheres with a secondary maximum in the tropics. The maxima are generally symmetric about the equator although slightly stronger and broader amplitude is observed in the Southern Hemisphere than its Northern Hemisphere counterpart. The latitude-altitude structures of DW1 T in the stratosphere compare very well with the Modern Era Retrospective analysis for Research and Applications (MERRA) data (Sakazaki et al., 2012). Phase plot shows both maxima have constant phase, which indicates that DW1 is trapped and cannot propagate upward in this region. This is due to the in situ excitation by $\mathrm{O}_{3}$ absorption of UV solar radiation (Forbes et al., 2006; Forbes and Garrett, 1976; Forbes and Wu, 2006; Zhang et al., 2006).

In the mesosphere, the latitudinal structure of DW1 in temperature is characterized with an equatorial maximum with the amplitude $\sim 28 \mathrm{~K}$ at $100 \mathrm{~km}$ and two secondary maxima located symmetrically in the mid-latitudes (near $\pm 35^{\circ}$ ) of each hemisphere. The equatorial maxima in eCMAM30 is slightly higher compared to the SABER data which has a maximum around $95 \mathrm{~km}$. This may be due to the underdamping of the wave (i.e., not enough eddy diffusivity) in eCMAM (Gan et al., 2014). The phase structure is symmetric over the equator and shows $180^{\circ}$ phase shift between the equatorial and midlatitude maxima. The decrease of the phase with height indicates the propagation of DW1 in this region. 

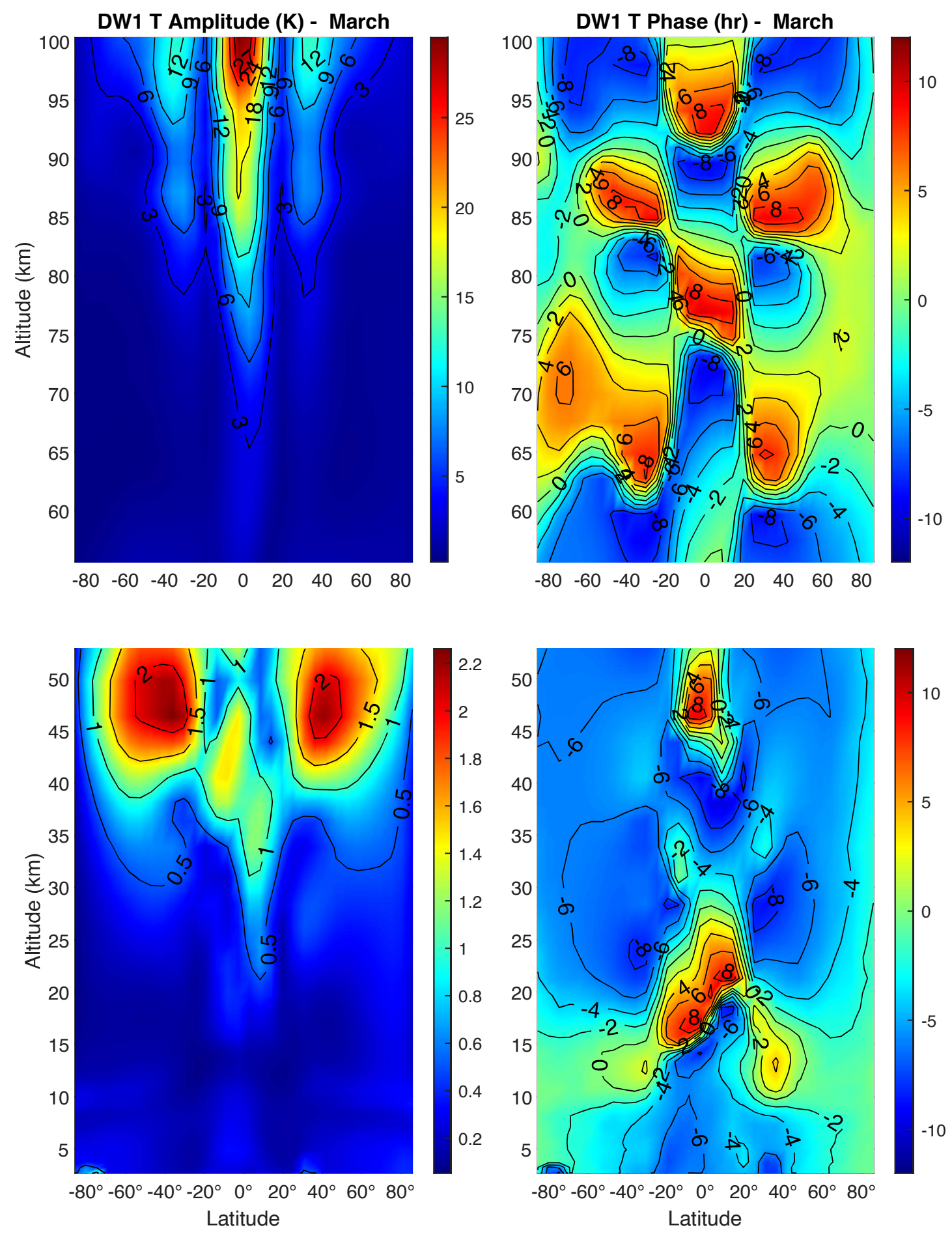

Figure 3.5. Climatological mean latitude-altitude structure of DW1 temperature amplitude (left) and phase (right) in the thermosphere (top panel), mesosphere (middle panel), and stratosphere (bottom panel) for March from the eCMAM30 (1979-2010). 
Figure 3.6 illustrates the seasonal variation of the temperature of the migrating diurnal tide DW1 at $45 \mathrm{~km}, 100 \mathrm{~km}$, and $165 \mathrm{~km}$. DW1 at $45 \mathrm{~km}$ shows a clear annual variation in the amplitude and maximizes during summer in each hemisphere. It is a broad feature from $20^{\circ}$ to $80^{\circ} \mathrm{N} / \mathrm{S}$ during summer months and this feature narrows to $30^{\circ}$ to $60^{\circ} \mathrm{N} / \mathrm{S}$ during winter months. This feature compares well with SABER (Gan et al., 2014). The amplitude of the DW1 at $100 \mathrm{~km}$ exhibits a strong semiannual variation with stronger amplitude during equinox and weaker amplitude during solstice. The March maximum is slightly stronger than that in September. This semiannual variation is eminent in both satellite and ground based observations (Vincent et al., 1998; Zhang et al., 2006; Gan et al., 2014). At $165 \mathrm{~km}$, the DW1 $\mathrm{T}$ amplitude maximizes over a broad latitude region from the high latitudes of one hemisphere to the other, with peak value occurring in the tropical and subtropical regions in both hemispheres.

From previous work and the above analysis, we are confident that the eCMAM30 can simulate tidal dynamics realistically and provide a very important tool for investigating the short-term tidal variability and its mechanisms in the stratosphere and MLT region. The eCMAM30 data used in this dissertation may be obtained by registering with the Canadian Centre for Climate Modeling and Analysis (CCCma) using the online form: http://climate-modelling.canada.ca/climatemodeldata/ application. shtml. 

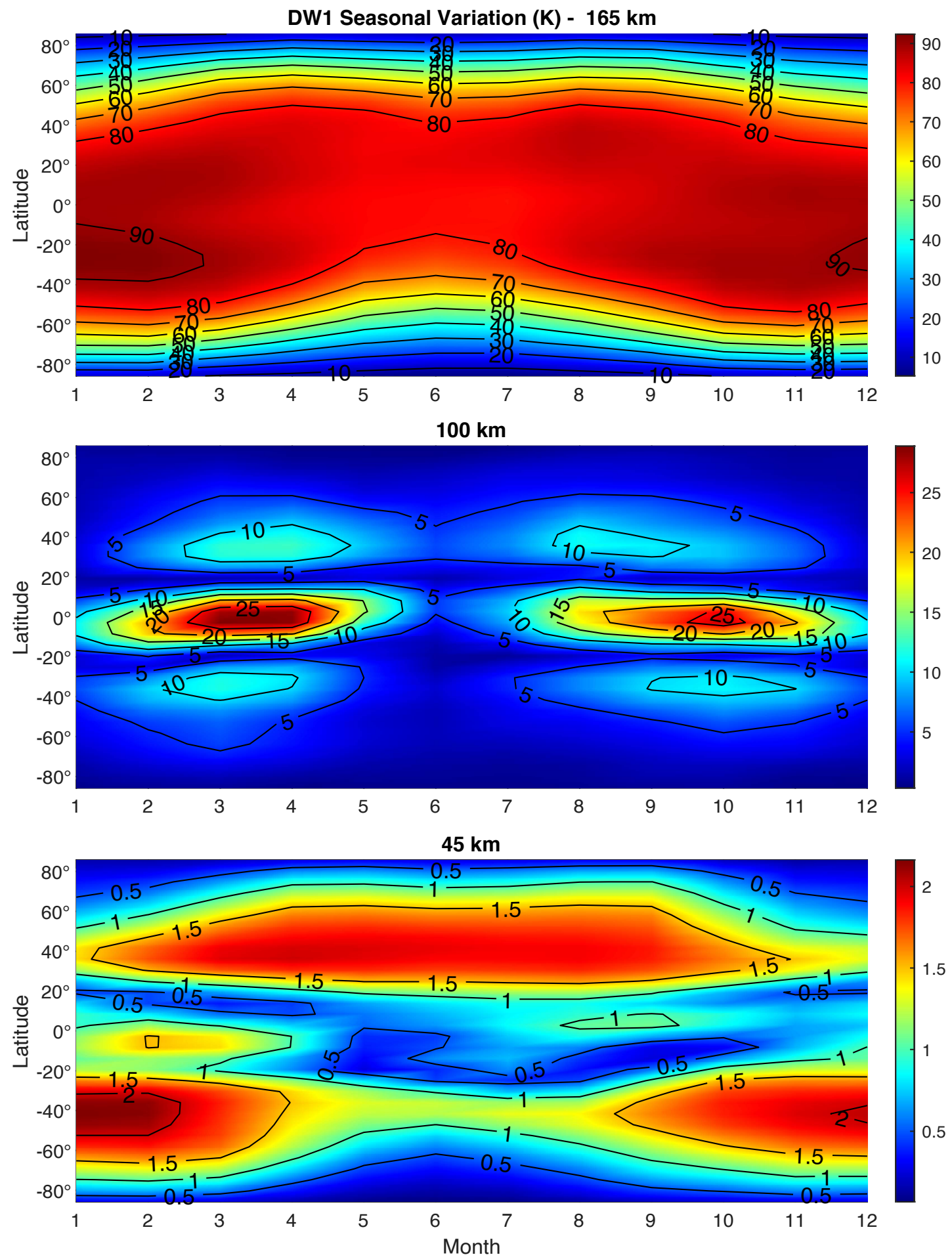

Figure 3.6. Seasonal variations of the DW1 T amplitude (K) at $165 \mathrm{~km}$ (top panel), $100 \mathrm{~km}$ (middle panel), and $45 \mathrm{~km}$ (bottom panel) from eCMAM30 as a function of latitude and month. 


\subsection{SABER Short-term Tidal Diagnostics}

This section is obtained from the Vitharana et al. (2019). SABER is one of the four instruments on NASA's TIMED satellite launched on 7 December 2001 (Russell et al., 1999). SABER is the first spaceborne instrument to provide systematic global measurement of atmospheric density and temperature as well as key atmospheric heating and cooling rates (Kramer, 2002). The primary goal of the SABER experiment is to provide the data needed to advance our understanding of the fundamental processes governing the energetics, chemistry, dynamics, and transport in the mesosphere and lower thermosphere. SABER uses a 10-channel broadband limb-scanning infrared radiometer covering 1.27 to $17 \mu \mathrm{m}$ spectral range and temperature profiles are retrieved from two $15 \mu \mathrm{m}$ and one $4.3 \mu \mathrm{m} \mathrm{CO}$ channels between 20 to $120 \mathrm{~km}$ with a $2 \mathrm{~km}$ vertical resolution (Remsberg et al., 2008). TIMED is a quasi-sun synchronous satellite with $74^{\circ}$ orbital inclination and sample the data at two local solar times per day. SABER always views on the anti-sun side of the spacecraft (perpendicular to the orbit). Latitude coverage extended from $53^{\circ} \mathrm{S}$ and $83^{\circ} \mathrm{N}$ or $53^{\circ} \mathrm{N}$ and $83^{\circ} \mathrm{S}$, depends on the orientation of the spacecraft with respect to the orbit, which changes by 180 degrees every 60 days. Figure 3.7 shows the SABER measurement track for 11 April 2008. The slow orbital precession rate $(\sim 12 \mathrm{~min} /$ day $)$ of the satellite results in full (24 hours) local time coverage every 60 days when both ascending and descending orbit node data are combined.

For SABER data, a 60-day running mean Fourier spectral diagnostics is needed to perform tidal decomposition (e.g., (Forbes et al., 2008; Gan et al., 2014; Huang et al., 2006; Mukhtarov et al., 2009; Pedatella et al., 2016; Remsberg et al., 2008). This 60-day averaging period is useful for studies of seasonal and interannual variability. However, it is too long to study tidal "weather", that is, short-term tidal variability on a time scale of days or weeks. Short-term tidal variability in nonmigrating tides can be resolved by employing daily tidal diagnostics from the "tidal deconvolution" 
method. The method developed by Oberheide et al. (2002) has been validated for studying short-term tidal variability using WINDII and SABER data (Lieberman et al., 2013, 2015; Pedatella et al., 2016).

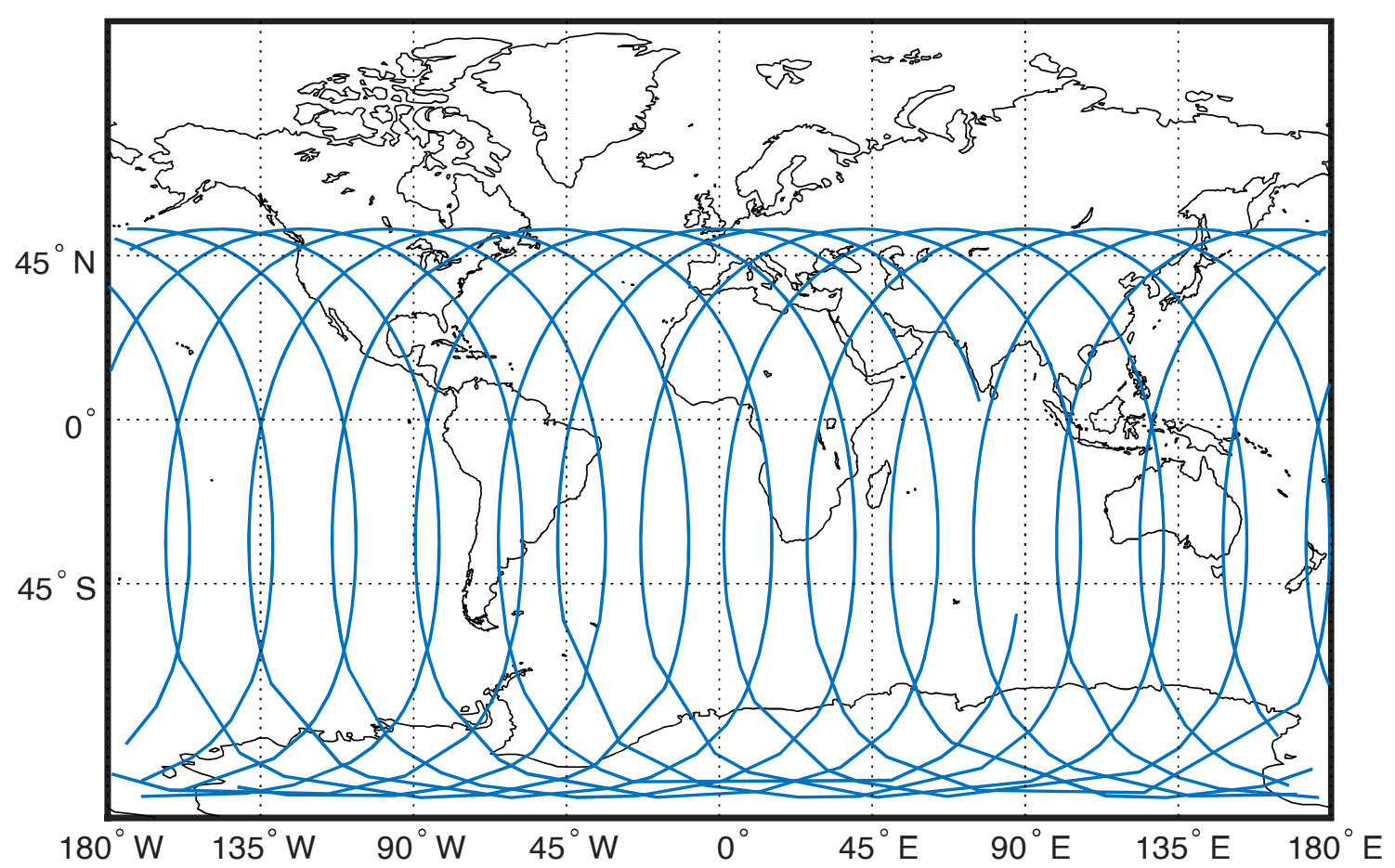

Figure 3.7. SABER measurement track for 11 April 2008 as a function of latitude and altitude.

For short-term tidal variability in migrating diurnal tide DW1, daily amplitudes and phases of DW1 can be retrieved from differences between daily zonal mean ascending and descending measurements that can be thought of as a global "snapshot" of the migrating diurnal tide (Hitchman and Leovy, 1985; Wallace and Hartranft, 1969; Wallace and Tadd, 1974; Ward et al., 1999). Note that SABER observes migrating tides at a given latitude as zonally symmetric features (Oberheide et al., 2003). Tidal perturbations from the background temperature $\bar{T}$ for the local solar time of the SABER observations on the ascending and descending orbit portions can 
be written as

$$
\begin{aligned}
& T_{a s c}=\bar{T}+T_{o} \cos \left(\omega t_{a s c}-\phi\right) \\
& T_{d s c}=\bar{T}+T_{o} \cos \left(\omega t_{d s c}-\phi\right)
\end{aligned}
$$

with migrating diurnal amplitude $T_{o}$, phase $\phi$, frequency $\omega=2 \pi / 24 \mathrm{~h}^{-1}$, and $t_{\text {asc }}$, $t_{d s c}$ as the local solar time of the observations on the ascending (asc) and descending (dsc) orbit nodes, respectively. The difference $\Delta T$ between Equations 3.1 and 3.2 becomes

$$
\begin{array}{r}
\Delta T=T_{a s c}-T_{d s c}=T_{o}\left(\cos \left(\omega t_{a s c}-\phi\right)-\cos \left(\omega t_{d s c}-\phi\right)\right) \\
=-2 T_{o} \sin \left(\omega \frac{\Delta t}{2}\right) \sin \left(\omega \frac{t_{a s c}+t_{d s c}}{2}-\phi\right)
\end{array}
$$

where $\Delta t=t_{a s c}-t_{d s c}$.

A limitation of the snapshot approach is that it does not provide sufficient information about the phase of the migrating diurnal tide. However, Lieberman (1991) and Oberheide et al. (2000) have both demonstrated that the amplitude and phase of the migrating diurnal tide information can be determined from the vertical structure of $\Delta t=12 \mathrm{~h}$ local solar time ascending-descending orbit differences. Amplitude curves are inferred from the absolute values of the local maxima and minima of the difference curve (as a function of altitude) and interpolated linearly in the vertical. Phase angles are inferred at the maxima, minima, and zero crossing of the difference curve, with the assumption of downward phase progression in local solar time. The solution is correct for $\Delta t=12 \mathrm{~h}$ because zonal mean (background) temperature and migrating semidiurnal tides (observed in the same phase) vanish in $\Delta T$.

However, there is potential for aliasing by semidiurnal tides when difference fields are not separated by 12 hours in local solar time. This is the case for SABER where 
the equatorial local time difference is 9 hours. The magnitude of this semidiurnal tidal aliasing depends on season, latitude, and altitude. SABER diagnostics using 60-day running mean Fourier fits indicates $\geq 10 \mathrm{~K}$ amplitude of the semidiurnal tide at altitudes $\geq 100 \mathrm{~km}$ in the $20^{\circ}-40^{\circ}$ latitude range between April and September in the Southern Hemisphere and October to March in the Northern Hemisphere, respectively, but comparatively small $(\leq 5 \mathrm{~K})$ amplitudes equatorward of $20^{\circ}$ (e.g. Akmaev et al. (2008)). Note that semidiurnal tidal amplitudes decrease rapidly towards lower altitudes at all latitudes, e.g., $\leq 5 \mathrm{~K}$ in the $20^{\circ}-40^{\circ}$ latitude range at $90 \mathrm{~km}$ (Pancheva et al., 2009). Equation 3.3 suggests that the semidiurnal aliasing can be estimated as the semidiurnal amplitude times $\sin \left(\frac{\pi}{6} \Delta t\right)$.

Depending on the yaw-associated spacecraft orientation, $\Delta t \approx 7$ hours or $\Delta t \approx 11$ hours at $20^{\circ}-40^{\circ}$ latitude, and $\Delta t \approx 9$ hours at the equator. This translates into $\sin \left(\frac{\pi}{6} \Delta t\right)=0.99,0.71$, and 0.26 , respectively. Therefore, semidiurnal aliasing is generally on the order of $\leq 1.2 \mathrm{~K}$ equatorward of $20^{\circ}$ but substantially larger poleward of $20^{\circ}$ at altitudes above $90 \mathrm{~km}$. The ascending-descending orbit node differencing is applied to $\pm 2.5^{\circ}$ latitude bins every $5^{\circ}$. Daily migrating diurnal tide definitions are averaged into four-day running means in order to mitigate artificial short-term variability originating from the fact that the ascending-descending orbit node differencing still assumes constant amplitudes and phases within one day of observations. Using a four-day window yields good agreement with ground-based observations during the Climate and Weather of the Sun-Earth System (CAWSES) tidal campaigns, as discussed by Ward et al. (2010).

The ascending-descending orbit node differencing provides continuous time series of amplitudes and phases up to $\sim 100 \mathrm{~km}$ altitude, apart from days when the satellite is changing its orientation with respect to the orbit ground-track (yaw, every 60 days) and days with data gaps in the temperature observations. Above $100 \mathrm{~km}$, however, gaps in the amplitude and phase time series become much more frequent because the 
uppermost altitude of recovered tidal amplitudes and phases is determined by the uppermost altitude of a minimum or maximum in $\Delta T$ in each latitude bin. Figure 3.8 shows the missing data in SABER DW1 at the equator in 2007. In this dissertation study, the statistical properties of DW1 tidal variability is investigated by using statistical tools such as multi-linear regression, auto-correlation, and correlation, etc. Hence it is important to fill the missing data gaps presented in SABER data before doing any further analysis. The following section describes the gap filling method used in this dissertation.

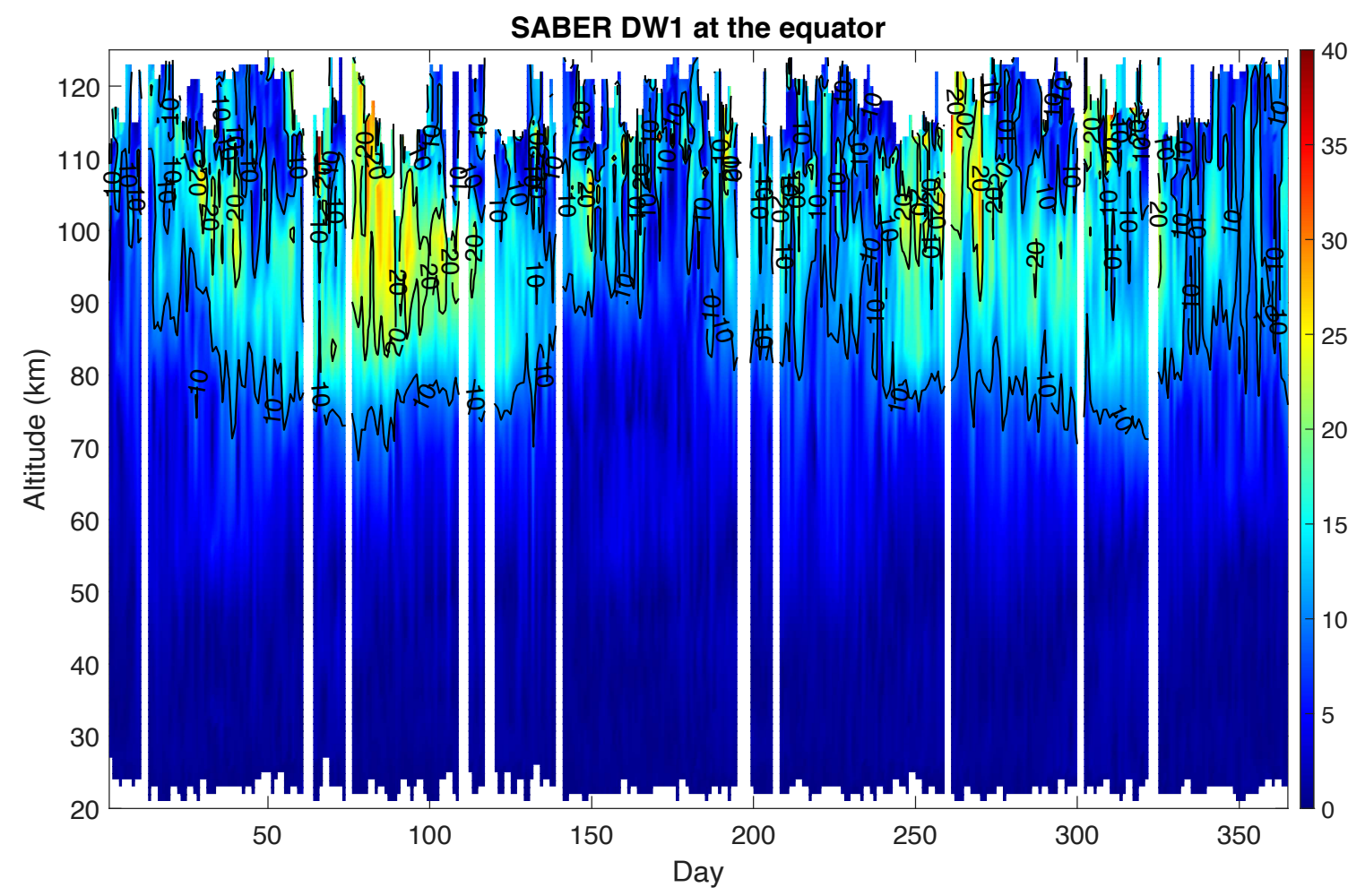

Figure 3.8. DW1 tidal amplitude as a function of altitude and day in 2007 from SABER. White space is missing data.

\subsection{Treatment of Data Gaps}

Atmospheric data are often unevenly sampled due to the spatial and temporal gaps in satellites and ground-based measurements. The techniques to fill the data gaps can be categorized as statistical methods (e.g., least square regression analysis), em- 
pirical methods (e.g., simple arithmetic averaging), and function fitting (e.g., polynomial interpolation) (Xia et al., 1999). Atmospheric parameters often have periodic oscillations, hence the Lomb-Scargle periodogram (Lomb, 1976; Scargle, 1982) is a commonly-used statistical tool for detecting and characterizing the periodic signals in an unevenly sampled time series (Altadill et al., 2003; Ford et al., 2006; Huang et al., 2018; Nozawa et al., 2005; Oberheide et al., 2009). Hence a gap filling method based on the Lomb-Scargle periodogram developed by Hocke and Kämpfer (2009) is employed. Lomb-Scargle periodogram can be viewed as a least-squares method and falls into the category of statistical methods. The procedure for the reconstruction of data gaps is illustrated in Figure 3.9 and described as follows.

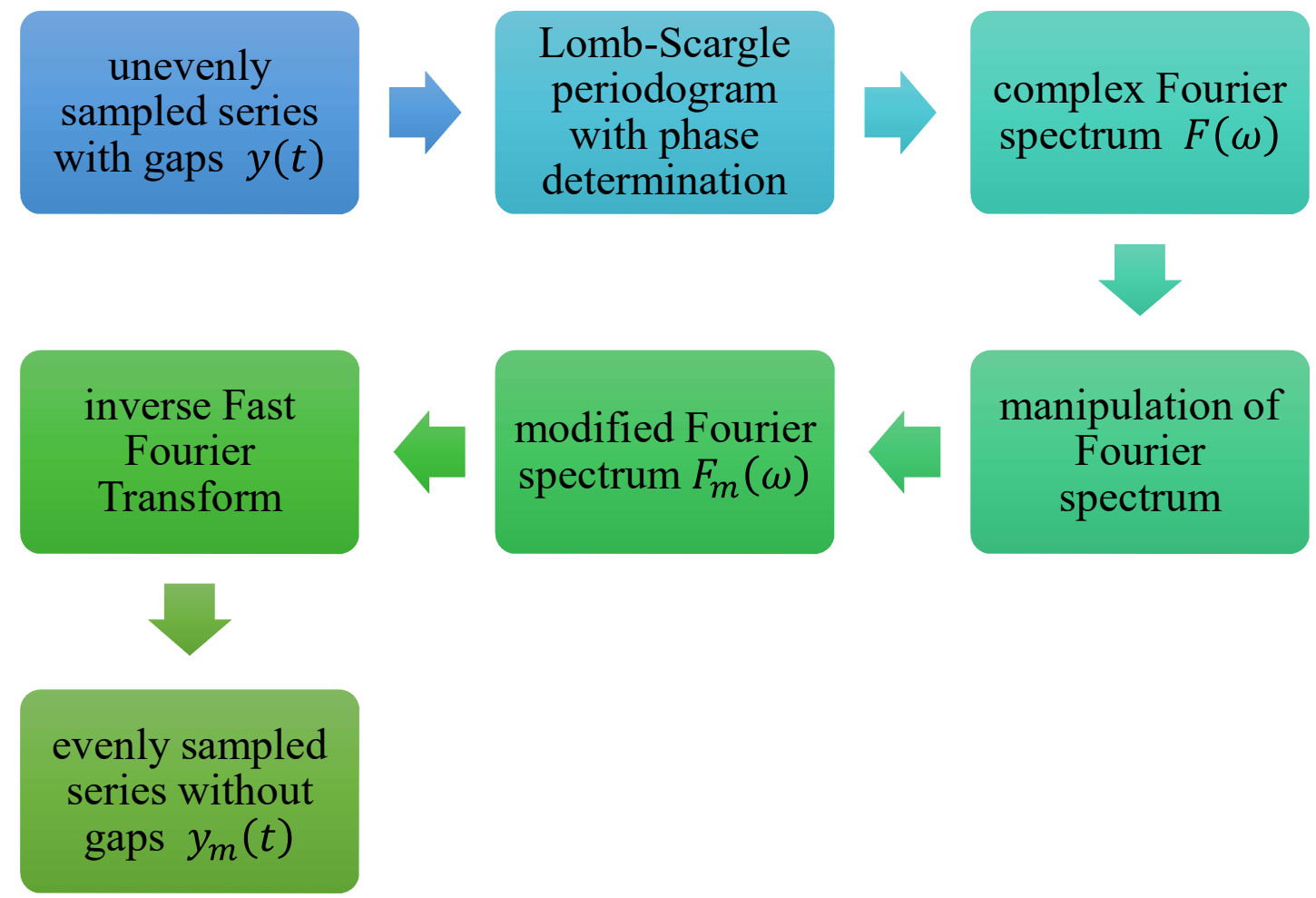

Figure 3.9. Flow chart of filling data gaps in SABER with the Lomb-Scargle method. This figure is a derivative of Figure 1 from Hocke and Kämpfer (2009), used under a Creative Commons Attribution 4.0 International Public License: https: //creativecommons.org/licenses/by/4.0/.

When unevenly sampled data are present in the time series $y(t)$ where $y_{i}$ taken at 
times $t_{i}$ with $i=1,2,3, \ldots, N$, the Lomb-Scargle periodogram is defined by (Lomb, 1976)

$$
P(\omega)=\frac{1}{2 \sigma^{2}}\left\{\frac{\left[\sum_{i=1}^{N}\left(y_{i}-\bar{y}\right) \cos \left(\omega t_{i}-\Theta\right)\right]^{2}}{\sum_{i=1}^{N} \cos ^{2}\left(\omega t_{i}-\Theta\right)}+\frac{\left[\sum_{i=1}^{N}\left(y_{i}-\bar{y}\right) \sin \left(\omega t_{i}-\Theta\right)\right]^{2}}{\sum_{i=1}^{N} \sin ^{2}\left(\omega t_{i}-\Theta\right)}\right\}
$$

where

$$
\bar{y}=\frac{1}{N} \sum_{i=1}^{N} y_{i}
$$

and

$$
\sigma^{2}=\frac{1}{N-1} \sum_{i=1}^{N}\left(y_{i}-\bar{y}\right)^{2}
$$

are respectively the mean and variance of the data. The time offset $\Theta$ is chosen as

$$
\Theta=\frac{1}{2} \arctan \left(\frac{\sum_{i=1}^{N} \sin \left(2 \omega t_{i}\right)}{\sum_{i=1}^{N} \cos \left(2 \omega t_{i}\right)}\right)
$$

to guarantee the time invariance of the computed spectrum. The offset depends only on the measurement times and vanishes when the times are equally spaced (Scargle, 1982).

Hocke and Kämpfer (2009) constructed the complex Fourier spectrum $F(\omega)$ using the Lomb-Scargle periodogram $P(\omega)$ and time offfset $\Theta$ information. The complex Fourier spectrum is given by

$$
F(\omega)=A_{F T} \cos \varphi_{F T}+i A_{F T} \sin \varphi_{F T}
$$

where the amplitude $A_{F T}$ of the complex Fourier spectrum is

$$
A_{F T}=\sqrt{\frac{N}{2} \sigma^{2} P(\omega)}
$$

and the phase $\varphi_{F T}$ of the complex Fourier spectrum is 


$$
\varphi_{F T}=\arctan \left(\frac{\sum_{i=1}^{N}\left(y_{i}-\bar{y}\right) \sin \left(\omega t_{i}-\Theta\right)}{\sum_{i=1}^{N}\left(y_{i}-\bar{y}\right) \cos \left(\omega t_{i}-\Theta\right)}\right)+\omega\left(\frac{t_{1}+t_{N}}{2}\right)+\Theta
$$

Once the complex Fourier spectrum is constructed, it can be modified (e.g., by removing the spectral components below a certain confidence limit). The real part of the inverse Fourier transformation of the modified Fourier Spectrum $F_{m}(\omega)$ provides the evenly spaced series $y_{m}(t)$ without gaps. Preliminary MATLAB program developed by Hocke and Kämpfer (2009) for the Lomb-Scargle reconstruction is taken from the website: http://www.atmos-chem-phys.net/9/4197/2009/ acp-9-4197-2009-supplement.zip.

SABER version 1.07 data from 2003 to 2010 and version 2.0 data from 2003 to 2017 are used in this dissertation that have been validated by Remsberg et al. (2008) and Dawkins et al. (2018), respectively. The SABER team ceased the production of version 1.07 data in 2013 and switched to version 2.0. Version 2.0 includes many algorithm improvements (e.g. the temperature retrieval algorithm for the mesosphere and chemical heating algorithms). The main difference between the two versions is the altitude coverage. SABER version 1.07 data stops at $120 \mathrm{~km}$ and version 2.0 data stops at $110 \mathrm{~km}$. Due to the reduced altitude coverage of the version 2.0 data, the uppermost altitude with more or less complete temporal coverage to resolve the shortterm tidal variability is about $100 \mathrm{~km}$. The comparison between version 1.07 and 2.0 data below $95 \mathrm{~km}$ shows that the results are pretty much identical between the two versions (see Appendix B). In Chapter 4, statistical properties of DW1 short-term tidal variability are studied between 2003 - 2010 using eCMAM30 and SABER data. SABER version 1.07 is used for the analysis, since it provides more altitude coverage. Chapter 5 presents the analysis of the total tidal variability of DW1. SABER version 2.0 is used in this chapter because it has longer temporal span than version 1.07. Figure 3.10 shows the missing data percentage in SABER DW1 v1.07 (top panel) between 2003 - 2012 and v2.0 (bottom panel) between $2003-2017$ as a function of 
altitude

SABER Missing Data Percentage

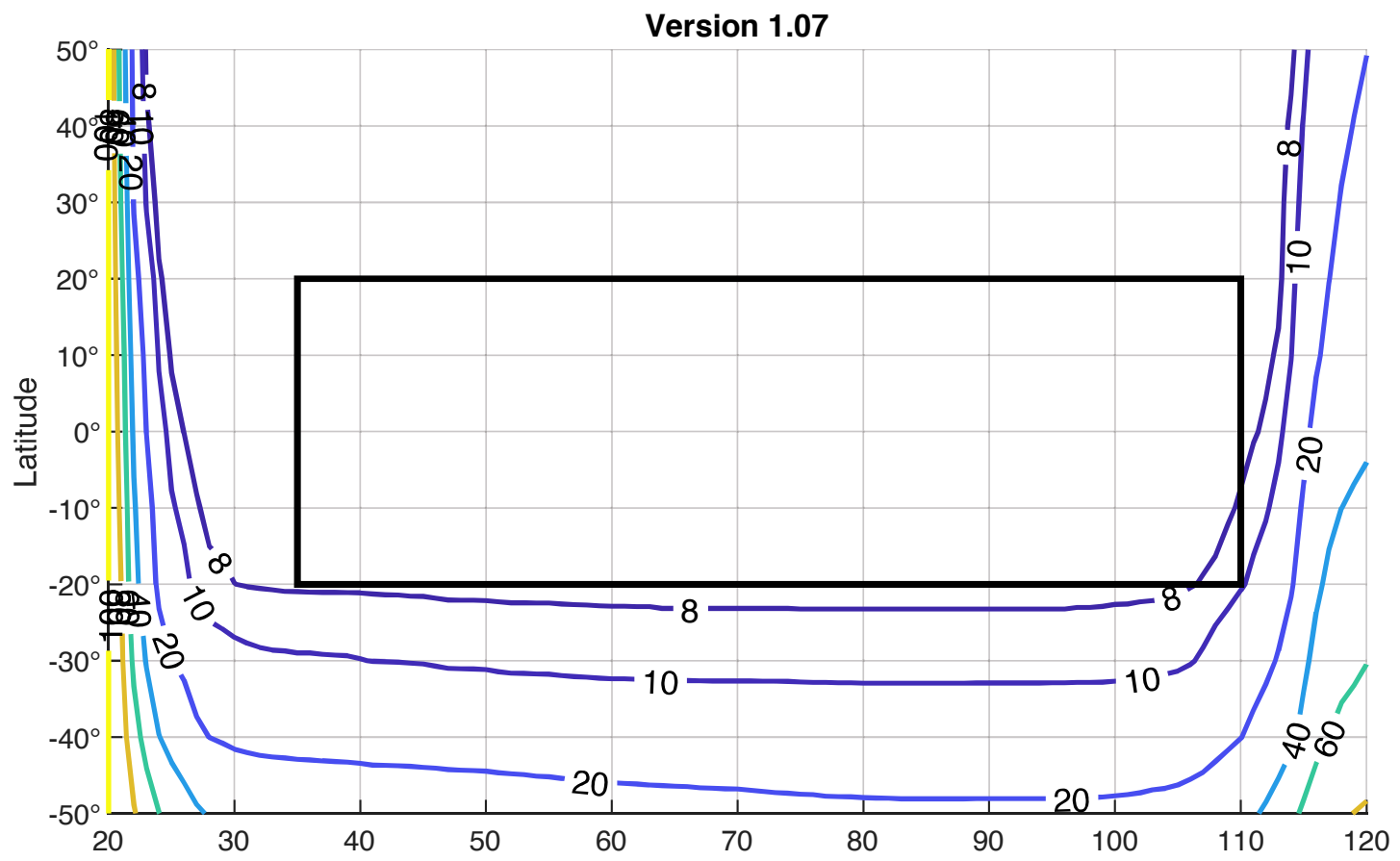

Version 2.0

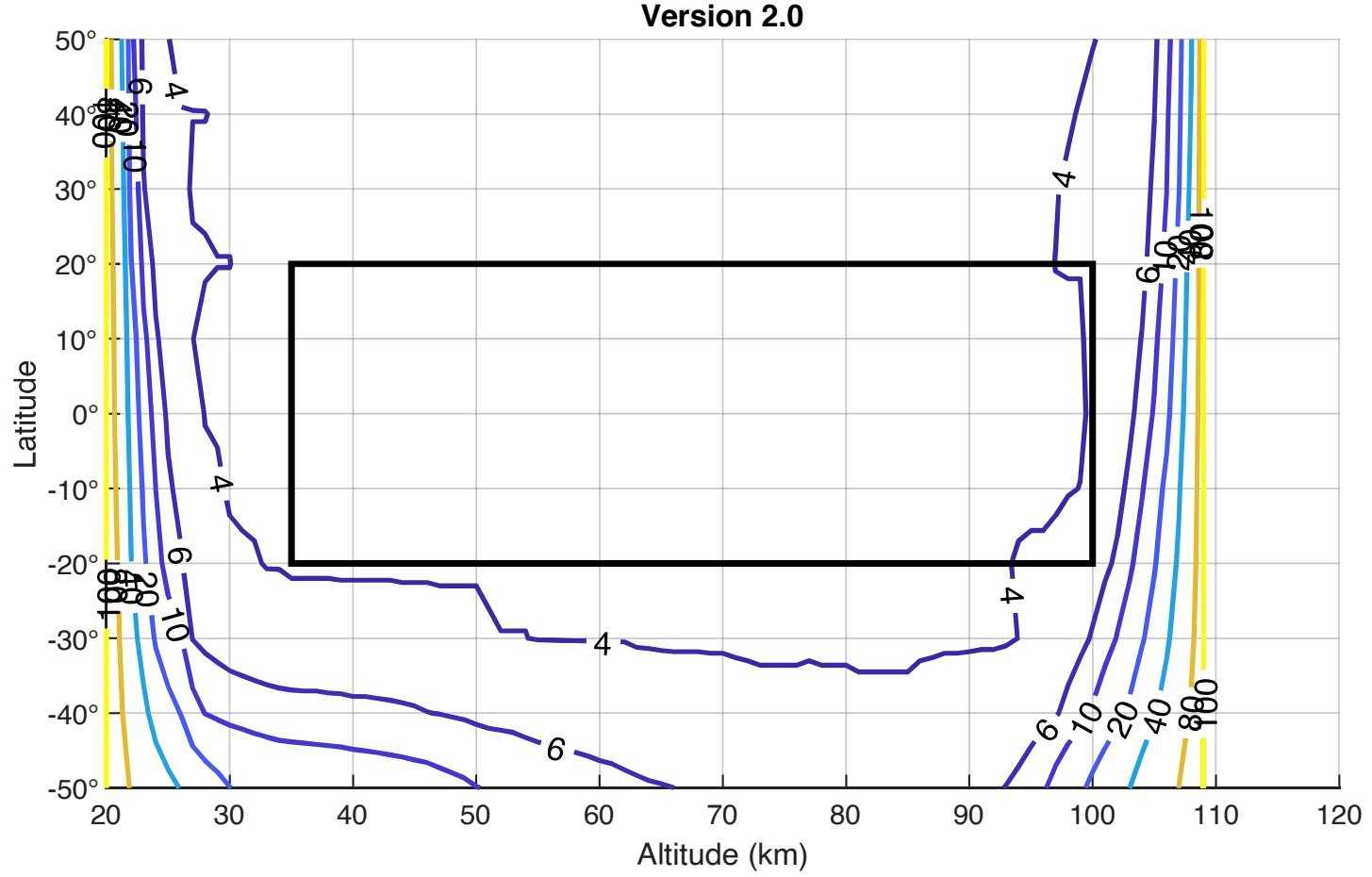

Figure 3.10. Missing data percentage in SABER DW1 version 1.07 (top) between 2003 - 2012 and version 2.0 (bottom) between 2003 - 2017 as a function of altitude and latitude. 
and latitude. SABER data is limited within 35 and $110(95) \mathrm{km}$ and $20^{\circ} \mathrm{N}$ to $20^{\circ} \mathrm{S}$ for SABER v1.07 (v2.0), the missing data ratio in this range is less than $8 \%(6 \%)$ and the majority of the results presented in this dissertation are at the Equator at 95 and $100 \mathrm{~km}$, which has a missing data ratio $<\sim 6 \%$ for v1.07 and $<\sim 4 \%$ for v2.0 and only the missing gaps in the original time series were replaced using the reconstructed time series. Hence do not expect the choice of data filling methods to have a significant impact on presented results. 


\section{CHAPTER 4}

\section{STATISTICAL MODELING OF TIDAL WEATHER IN THE MESOSPHERE AND LOWER THERMOSPHERE}

This chapter presents the investigation of the statistical characteristics of the tidal weather in DW1 (variability periods $<30$ days) using temperature data from the eCMAM30 and SABER. SABER version 1.07 data are used in this study. Both the eCMAM30 and SABER data are interpolated to have the same resolution in geometric height $(3 \mathrm{~km})$ and latitude $\left(5^{\circ}\right)$. The daily tidal amplitude is averaged with a five-day window and shifted by one day for both datasets. The overlapped time span between eCMAM and SABER is between January 2003 and June 2010. Tidal weather studied here is a deviation from the tidal climatology. For the current literature reviews, refer Section 1.3.4 in Chapter 1.

The results presented in this chapter are mostly from the Vitharana et al. (2019), published in the Journal of Geophysical Research: Atmospheres and can be found as:

Vitharana, A., Zhu, X., Du, J., Oberheide, J., \& Ward, W. E. (2019). Statistical modeling of tidal weather in the mesosphere and lower thermosphere. Journal of Geophysical Research: Atmospheres, 124, 9011-9027. https://doi.org/10.1029/ 2019JD030573

The chapter is organized as follows. Section 4.1 presents the DW1 tidal climatology (variability periods $>30$ days) and tidal weather (variability periods $<30$ days), and Section 4.2, illustrate the statistical properties of tidal weather, e.g., the 
auto- and spatial correlations. Based on these statistical properties of tidal weather, a hierarchy of statistical models developed to predict the tidal weather and results are presented in Section 4.3. Section 4.4 presents the results of the prediction accuracy of the proposed models and the stability of the model coefficients with different sensitivity tests. The tidal weather forecast from eCMAM and comparison of eCMAM prediction with SABER described in Section 4.5 and Section 4.6, respectively. Section 4.7 presents discussions and conclusions.

\subsection{DW1 Tidal Climatology and Tidal Weather}

The time series are seperated into climatological mean, long-term variability (variability periods $>30$ days), and short-term variability (variability periods $<30$ days) by applying a Fourier filter. The Fourier transformation is applied to the 30 years of eCMAM data and 9 years of SABER data. Fourier transformed time series are modified by removing the tidal variability $>30$ and then inverse Fourier transformation is applied to get the tidal weather time series. Figure 4.1 (first panel) shows the climatological mean amplitude of the DW1 from January 2003 to June 2010 as a function of latitude and height $(35-110 \mathrm{~km})$ in eCMAM (left) and SABER (right). The contour interval is $2 \mathrm{~K}$. The white dashed lines in the eCMAM plots represent the cutoff latitudes in $\operatorname{SABER}\left(20^{\circ} \mathrm{S}\right.$ and $\left.20^{\circ} \mathrm{N}\right)$. The mean structure of DW1 in eCMAM is characterized by an equatorial maximum and two secondary maxima located symmetrically in the mid-latitudes (near $\pm 35^{\circ}$ ) of each hemisphere above $60 \mathrm{~km}$. The equatorial maximum is located between 100 and $110 \mathrm{~km}$ in eCMAM, with mean maximum amplitude of 18 to $20 \mathrm{~K}$. The secondary maxima in the mid-latitudes have mean maximum amplitudes of $8 \mathrm{~K}$ in eCMAM.

There is also a maximum between 90 and $100 \mathrm{~km}$ over the equator in the SABER amplitudes, but with weaker mean amplitude $(12-14 \mathrm{~K})$ than the eCMAM. Gan et al. (2014) discussed the $\sim 10 \mathrm{~km}$ height difference between maximum amplitude 
around the equator in SABER and eCMAM, and suggested that the eCMAM may be under-damping the wave above $60 \mathrm{~km}$. They also compared the climatology of the DW1 amplitude in the eCMAM to SABER with the traditional least square harmonics fitting method and found that the eCMAM DW1 amplitude maximum over the equator is about 30\% larger in March than SABER but in agreement in July.

Figure 4.1 (second panel) shows the long-term (variability periods $>30$ days) deviation from the climatological mean amplitude for DW1 in 2009 as a function of height in both eCMAM (left) and SABER (right). The semiannual oscillation is evident in both datasets, although it appears to be stronger in eCMAM than SABER. The third panel shows the corresponding short-term variability in 2009, e .g., tidal weather (variability periods $<30$ days), and there are significant positive and negative oscillations ( $\pm 7 \mathrm{~K}, 30-50 \%$ of the mean amplitude) in both datasets above $80 \mathrm{~km}$. The positive and negative oscillations extend downward to $35 \mathrm{~km}$.

Figure 4.1 (fourth panel) shows the wavelet analysis (Torrence and Compo, 1998) of tidal weather (variability periods $<30$ days) at the equator and $100 \mathrm{~km}$ from 2003 to 2010 in both eCMAM (left) and SABER (right). Regions within the solid black lines have passed the significance test. There is a rich spectrum of short-term tidal variability between periods of 8 to 30 days in both datasets. The drawback of the wavelet analysis is that it only shows variability with periods of $2^{n}$, where $n$ is an integer.

\subsection{Statistical Properties of Tidal Weather}

The short-term variability is the focus of the remainder of the chapter, for example, tidal weather (Figure 4.1 third panel), by studying its statistical properties and attempting to develop models to simulate and predict tidal weather. For the statistical properties of tidal weather, the auto-correlation (self-memory) and spatial 

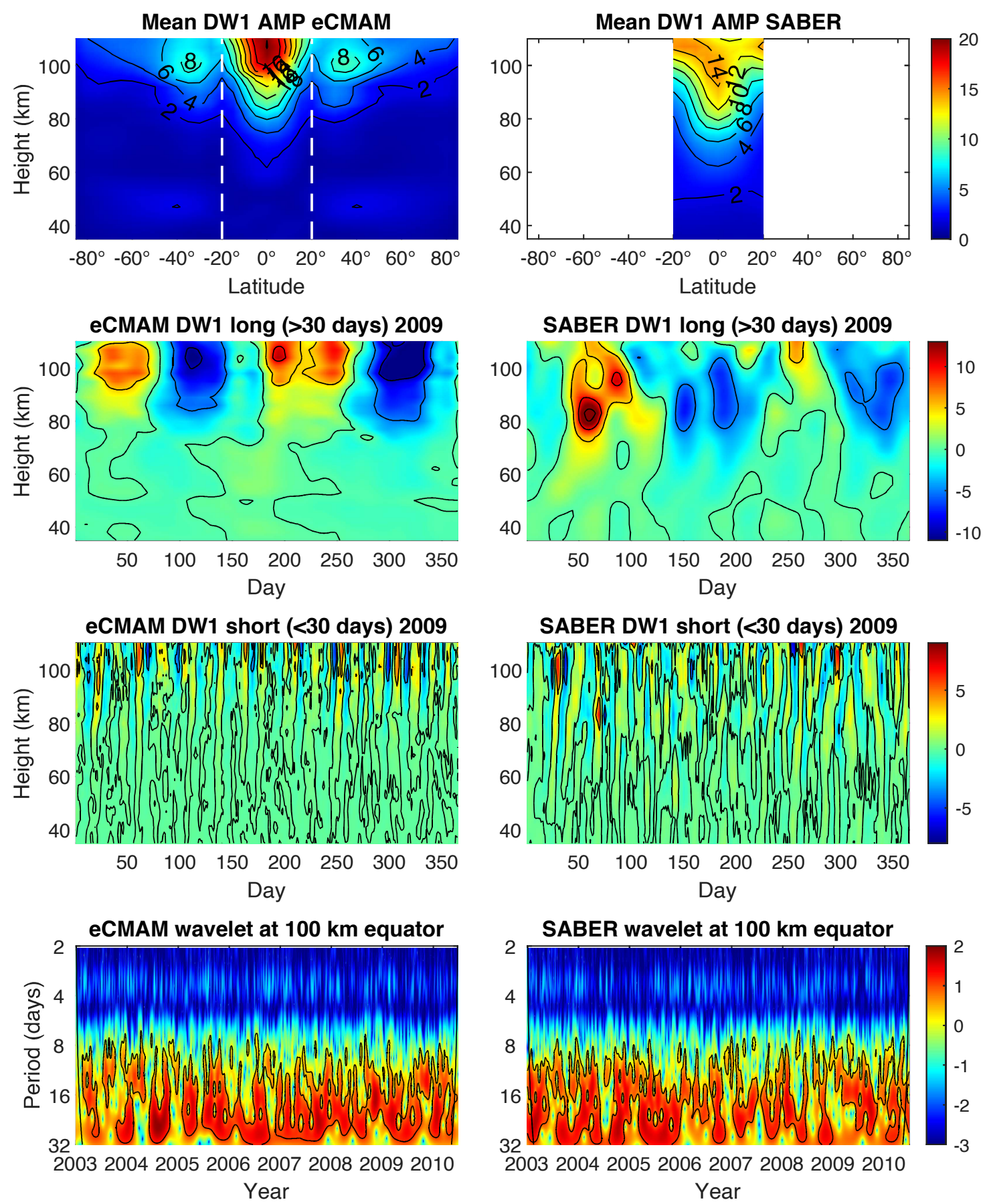

Figure 4.1. First panel: climatology mean amplitude $(\mathrm{K})$ of the DW1 $\mathrm{T}$ from January 2003 to June 2010 in the (left) eCMAM and (right) SABER. Second panel: long-term (periods $>30$ days) anomaly from the mean as a function of height and day in 2009 in eCMAM (left) and SABER (right). Third panel: Same as the second panel but for the short-term (periods $<30$ days) anomaly, for example, tidal weather in 2009. Fourth panel: Wavelet analysis of tidal weather at the equator and $100 \mathrm{~km}$ as a function of time (2003-2010). 
correlations (how tidal weather at a single location is correlated with tidal weather at neighboring latitudes and heights) are examined. This information is necessary for the development of the statistical models. The auto-correlation is used to identify the time scales over which the time series remains correlated and thus the training period for the statistical models. The spatial correlation information is used to test if it will provide additional information to significantly increase the forecast accuracy.

The auto-correlation analysis for tidal weather at $100 \mathrm{~km}$ and the equator is illustrated for eCMAM and SABER in Figure 4.2 (top two panels). The auto-correlation is calculated based on time series from January 2003 to June 2010 in both datasets. Periodicity in the auto-correlation of both eCMAM and SABER occurs at approximately 23 days, i.e., the time series loses correlation with itself after $\sim 23$ days. To check if a similar lag is present at other heights, and if this lag is sensitive to the length of the time series (Figure 4.2 third panel), the auto-correlation is calculated for time series of 100 days in eCMAM starting from January 1, 1979. The same calculation is repeated by increasing the time series length by a 100-day increment until the end of the time series (June 30,2010) from 35 to $100 \mathrm{~km}$. Although there is substantial variability ( $23-30$ days) with data lengths less than 5000 days, the optimal lag stabilizes and converges to $\sim 23$ days at all heights for longer data lengths. The average auto-correlation from all heights for both eCMAM and SABER is shown in Figure 4.2 (fourth left panel). The eCMAM (blue line) clearly converges to the lag of 23 days as more data points are included in the auto-correlation calculation. Due to SABER's shorter data length, the convergence to an optimal lag is less clear than with the eCMAM analysis but shows similar values (23-26 days). The autocorrelation lag with 6000 days of data is calculated and tested for different starting points (moving forward by 100 days) at all the heights. The distribution of the lag is shown as a histogram in Figure 4.2 (fourth right panel). The mean of the distribution is centered at 23-days, and the spread is between 19 to 26 days. The auto-correlation 
of 23 days is adapted as the training period for the statistical models.

Figure 4.3 (top panel) shows the correlations of tidal weather at the equator and a particular height with tidal weather at all the heights for eCMAM (left) and SABER (right) from January 2003 to June 2010. The correlations on the diagonal axis are 1 since the time series is correlating with itself. The diagonal acts as a symmetry axis since, for example, the correlations between $100 \mathrm{~km}$ and $60 \mathrm{~km}$ is the same as the correlation between $60 \mathrm{~km}$ and $100 \mathrm{~km}$. In eCMAM, strong and positive correlations ( $>0.6$, shades of red) are present within $5 \mathrm{~km}$ below $60 \mathrm{~km}$ altitude, then increase to $10 \mathrm{~km}$ from 60 to $90 \mathrm{~km}$ altitude and continue increasing to $20 \mathrm{~km}$ from 90 to $110 \mathrm{~km}$ altitude. Interestingly, there is a butterfly feature of relative high correlation between 55 and $65 \mathrm{~km}$ in the eCMAM, which is not present in SABER. Compared to the increasing correlation range with altitude in the eCMAM, the correlation in SABER is relatively invariant with altitude, and strong and positive correlations are present over a broader altitude range $(\sim 15-20 \mathrm{~km})$. The wider correlation range in SABER is likely to be related to the data processing method, which requires data from multiple neighboring altitudes to retrieve information at a single altitude. The strong height correlation is maybe related to the vertical wavelength of DW1, which is 20-25 km in the MLT region. The reason for varying range of stronger correlation as a function of height in eCMAM is unknown. One possible reason is that DW1 presents a different latitudinal structure in the stratosphere due to trapped Hough modes (see Gu and Du, 2018, fig. 1). The Hough mode composition in the stratosphere is more complicated than that in the MLT region where the symmetric Hough mode $(1,1)$ is dominant.

Figure 4.3 (bottom panel) shows spatial correlations of tidal weather at $100 \mathrm{~km}$ and a particular latitude and tidal weather at all latitudes in eCMAM (left) and SABER (right). The latitude range in the eCMAM analysis extends from $87.5^{\circ} \mathrm{N}$ to $87.5^{\circ} \mathrm{S}$ while the SABER analysis is limited to the latitude range between $20^{\circ} \mathrm{N}$ 

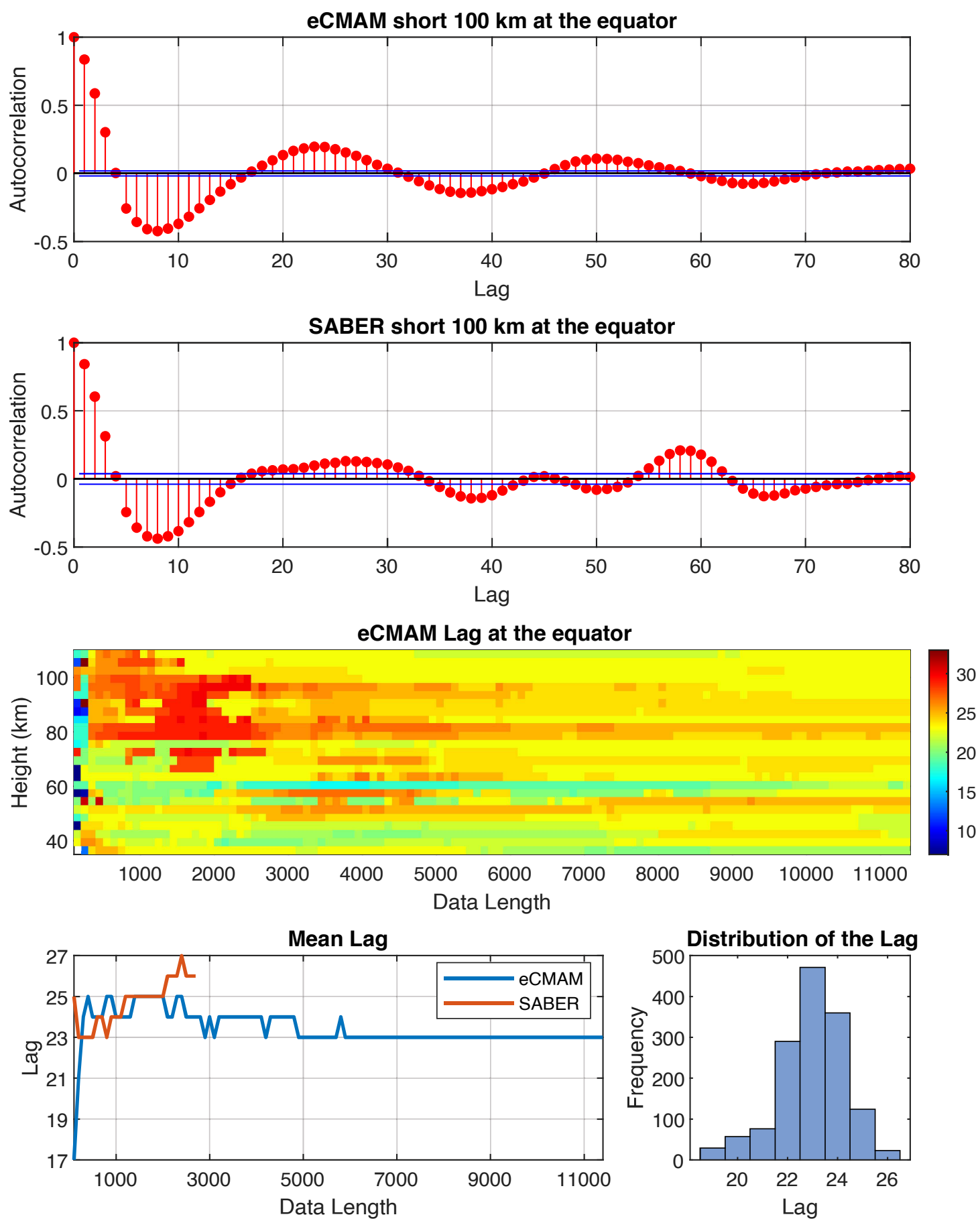

Figure 4.2. First panel: Auto-correlation of eCMAM DW1 tidal weather amplitude at $100 \mathrm{~km}$ and the equator. Second panel: Same as the first panel but for the SABER. Third panel: Auto-correlation of eCMAM DW1 tidal weather amplitude as a function of height and input data length. Fourth left panel: Average auto-correlation in eCMAM (blue line) and SABER (red line) as a function of the data input length. Fourth right panel: Histogram of auto-correlation lag for 6000-day input data length. 
and $20^{\circ} \mathrm{S}$. In the equatorial region, the tidal weather of DW1 is positively correlated ( $>0.6)$ with that of $2-3$ adjacent latitudes bins on either side of the latitude bin of interest, (corresponds to $10-15^{\circ}$ ). The latitudinal structure of DW1 at this altitude
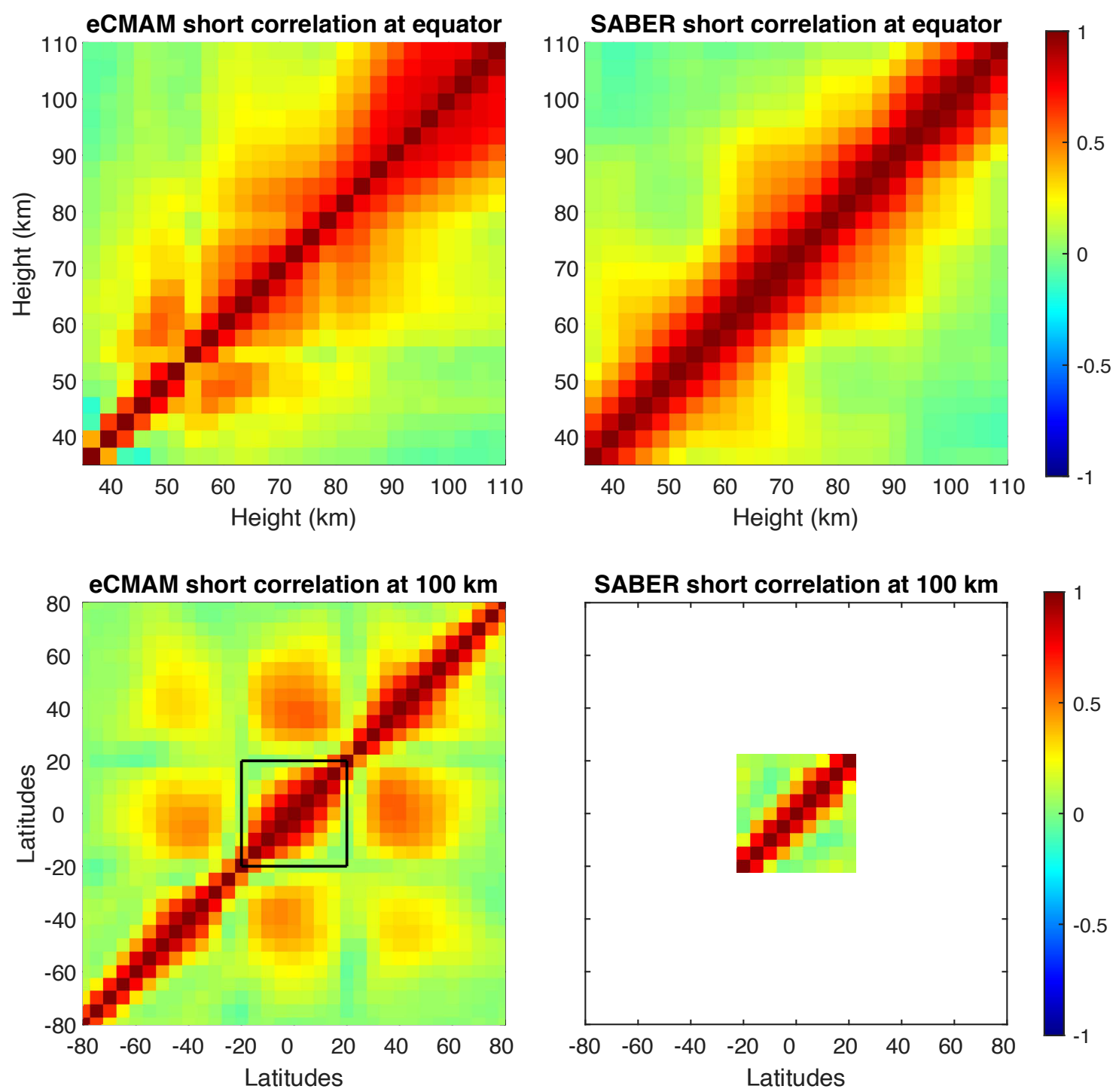

Figure 4.3. Top panel: Spatial correlations between DW1 tidal weather at a particular height and DW1 tidal weather at all heights $(35-100 \mathrm{~km})$ at the Equator for the eCMAM (left) and SABER (right). Bottom panel: Same as top panel, but for spatial correlations between latitudes at $100 \mathrm{~km}$.

(Figure 4.1) is characterized by one equatorial maximum and two secondary maxima in the subtropics. The correlations between the equatorial maximum and the sub- 
tropical maxima form the diamond-shaped correlations in the eCMAM and are in the range of $0.5-0.6$. The two subtropical maxima are also positively correlated but the correlation is weaker at $0.3-0.4$. The correlations in SABER weather for the equatorial maximum are slightly weaker than that in the eCMAM. Moreover, positive correlations in this case are only present $1-2$ neighboring latitudes, which corresponds to $\sim 10^{\circ}$. The strong latitudinal correlation is related to the latitudinal structure of the tide.

\subsection{Statistical Models of Tidal Weather}

An autoregressive (AR) model is a statistical technique that is used to analyze time varying processes in nature, economics, etc. An autoregressive model predicts future behavior of a variable using a linear combination of its past values. For example, in an $A R(1)$ process, current value is depending on the immediately preceding value and $A R(2)$ process, current value is based on the combination of the previous two values. Now, a hierarchy of statistical models are proposed with increasing complexity and more demanding computation power to simulate and predict tidal weather based on the statistical properties presented in Section 4.2. Three autoregressive models are proposed as follows.

\subsubsection{Model 1: Single Time Series Auto-Regression (AR) Model}

The AR time series model is fit at each height and latitude independently. The lag is determined from the auto-correlation and is fixed to be 23 for DW1 tidal weather. Each time series is modeled as:

$$
Y_{t}=C_{1}+\sum_{i=1}^{23} A_{i} Y_{t-i}+e_{1}
$$

The tidal variability $Y$ at time $t$ depends on a linear combination of the previous 23 days of data. It can be seen that there are 24 coefficients that need to be estimated, 
including mean $C_{1}$ and coefficients $A_{i}$, where $i=1, \ldots, 23 . e_{1}$ is the residue. In this study, since this study models tidal weather anomaly, mean $C_{1}$ is zero in this case.

\subsubsection{Model 2: Vector Auto-Regressive (VAR) model}

Since high correlations between neighboring heights and latitudes are observed, a spatial-temporal model could boost prediction accuracy if the neighboring time series provide additional information. Here a model is presented that simulates tidal weather at all heights (26 height levels) simultaneously. An individual time series is no longer independent but is a function of other time series. All neighboring heights are used as data input for predicting the future value at a particular height. A vector auto-regressive model is constructed to provide the fit:

$$
\begin{aligned}
{\left[\begin{array}{c}
Y_{1, t} \\
Y_{2, t} \\
\vdots \\
Y_{26, t}
\end{array}\right]=} & {\left[\begin{array}{c}
C_{1} \\
C_{2} \\
\vdots \\
C_{26}
\end{array}\right]+\left[\begin{array}{cccc}
A_{1,1}^{1} & A_{1,2}^{1} & \ldots & A_{1,26}^{1} \\
A_{2,1}^{1} & A_{2,2}^{1} & \ldots & A_{2,26}^{1} \\
\vdots & \vdots & \ddots & \vdots \\
A_{26,1}^{1} & A_{26,2}^{1} & \ldots & A_{26,26}^{1}
\end{array}\right]\left[\begin{array}{c}
Y_{1, t-1} \\
Y_{2, t-1} \\
\vdots \\
Y_{26, t-1}
\end{array}\right]+\ldots } \\
& +\left[\begin{array}{cccc}
A_{1,1}^{23} & A_{1,2}^{23} & \ldots & A_{1,26}^{23} \\
A_{2,1}^{23} & A_{2,2}^{23} & \ldots & A_{2,26}^{23} \\
\vdots & \vdots & \ddots & \vdots \\
A_{26,1}^{23} & A_{26,2}^{23} & \ldots & A_{26,26}^{23}
\end{array}\right]\left[\begin{array}{c}
Y_{1, t-23} \\
Y_{2, t-23} \\
\vdots \\
Y_{26, t-23}
\end{array}\right]+\left[\begin{array}{c}
e_{1} \\
e_{2} \\
\vdots \\
e_{26}
\end{array}\right]
\end{aligned}
$$

In Model 2, only spatial correlations among heights are considered and this model requires $26 \times 26 \times 23+26=15,574$ parameters to be estimated. eCMAM has sufficient data length $(26$ height levels $\times 31.5$ years of eCM AM data $\times 365$ days of tidal data $=$ $298,835)$ to estimate these many parameters. One could build a full model in which all latitudes and heights are considered, but a significantly larger number of coefficients would be added thus making it computationally more expensive. 


\subsubsection{Model 3: Parsimonious Vector Auto-Regressive (PVAR) Model}

A parsimonious VAR model is a model between Model 1 and Model 2, which can be built considering only highly correlated time series in both latitudes and heights. Four thresholds are proposed and tested in this paper with high correlation being considered to be: $>0.2,>0.4,>0.6$, and $>0.8$ respectively. For time series at height $i$, if the included neighboring time series are $Y_{i, 1}, Y_{i, 1+1}, \ldots, Y_{i, n}$, the model is described as following:

$$
Y_{i, t}=C_{1}+\sum_{p=1}^{23} \sum_{k=i, 1}^{i, n} A_{1, k}^{p} Y_{k, t-p}+e_{1}
$$

Here, $n$ represents the number of time series that passes the threshold.

\subsection{Obtain Model Coefficients}

The proposed models are fit using DW1 tidal weather amplitude time series (input data) to calculate the coefficients. Once the coefficients are obtained, information at $t+1$ is calculated based on information from previous time lags and spatial relationships and then comparing the prediction with the original eCMAM input. $23-$ day of tidal weather amplitude prior to the prediction day (one day) are needed as input for the models in order to predict the tidal weather amplitude at one day. This process is repeated for each day and the squared correlation coefficient ( $R^{2}$ values) between the original tidal weather time series and the predicted one-day tidal weather gives the prediction accuracy. The averaged $R^{2}$ values for one-day prediction over all height levels between 35 and $110 \mathrm{~km}$ are used to assess the proposed three models described in section 4.3. The prediction test period in this paper is two years from June 30, 2008 to June 30, 2010. The significance test is done by performing Monte Carlo white noise experiments 1000 times. These time series have the same length, mean and standard deviation as our time series. White noise is surely not correlated with our time series. 
The $R^{2}$ values are calculated between the white noise and tidal weather time series 1000 times, the mean $R^{2}$ is 0.03 , which corresponds to a correlation coefficient of 0.17. The $95 \%$ range for the 1000 experiments is 0.02 to 0.04 . Therefore, $R^{2}$ is $95 \%$ significant if above 0.04 .

First, the robustness of these coefficients are investigated as a function of input data length. The input data length is the number of data points that are used to find the coefficients for the models. In another word, how many days of data as input is needed for the calculation of these coefficients? 120 days prior to June 30, 2008 is used as the starting point to obtain these coefficients for the AR model and increase the input data length to 180-, 365-, 500-, 1000-, 2000-, 3000-, 4000-, and 5000-days prior. 120 is not the minimum length required for an $A R(23)$ model, but rather a minimum of 47 days. The longer the data length used for coefficient estimation, the more stable the coefficients are (as shown later in Figure 4.7). The minimum input data length required is different for models with varying complexity. The more neighboring heights/latitudes are involved, the more coefficients need to be estimated and thus more input data longer data input length is needed. A similar test is done on the VAR and PVAR models. The tidal weather is predicted from June 30, 2008 to June 30, 2010 using these different sets of coefficients and calculated the averaged $R^{2}$ values over all heights. Figure 4.4 illustrates the results and shows the average $R^{2}$ values as a function of different input data length (120 - 5000 days of data) for the different models. The bottom panel of Figure 4.4 is a magnified view for input data length of $2000-5000$ days.

The prediction accuracy is relatively invariant $\left(R^{2}=0.8-0.9\right.$, correlation coefficients $=0.9-0.95)$ as a function of the input data length for the simplest AR model (blue curve). For both the PVAR and VAR models, the prediction accuracy initially increases sharply with longer input data lengths and then becomes invariant, which implies the longer the data length used for coefficient estimation, the more stable the 

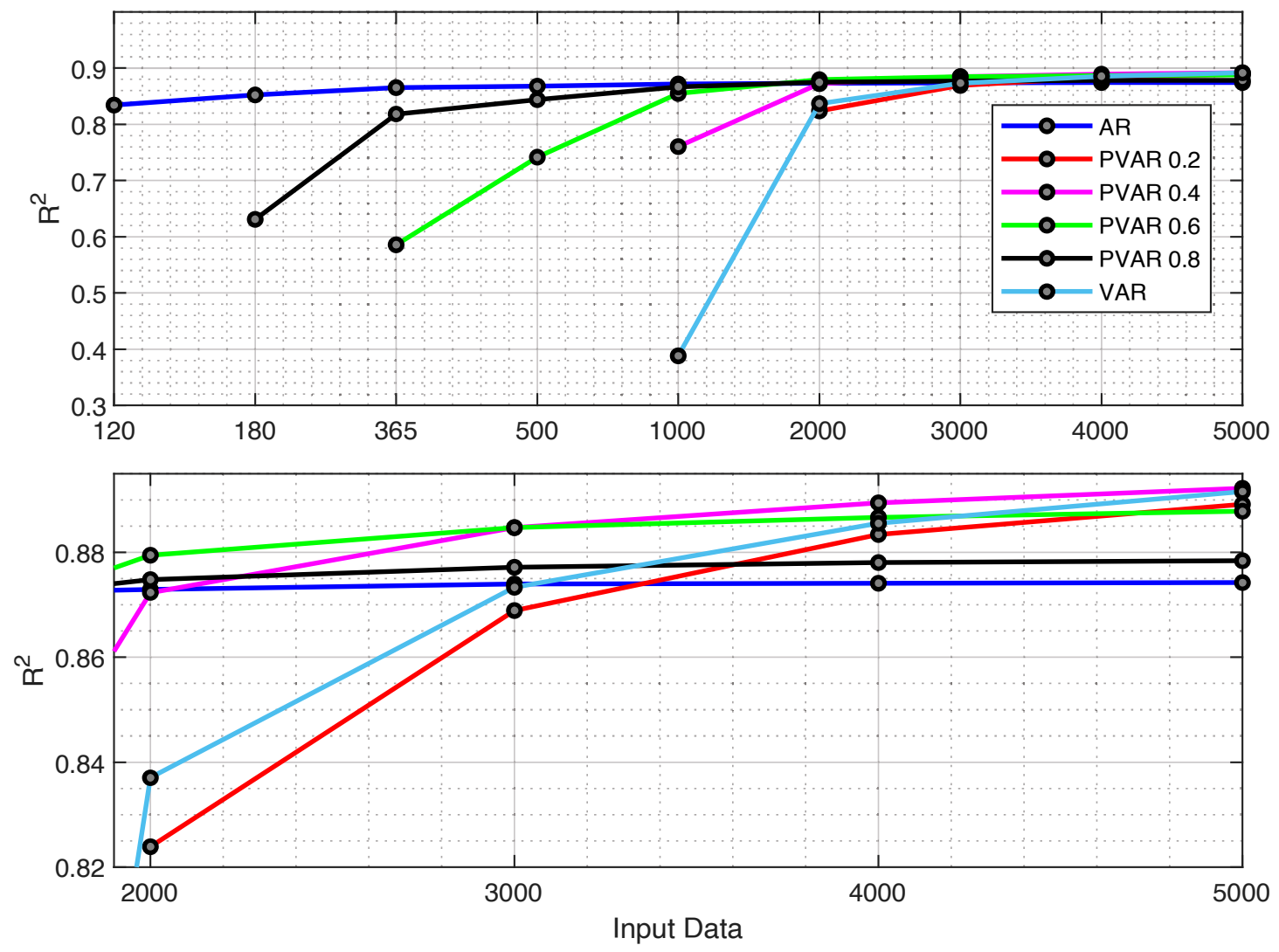

Figure 4.4. Prediction accuracy test of AR, VAR and PVAR models using $R^{2}$ values as a function of different input data length used to calculate model coefficients.

coefficients are. With 2000-day and above input data lengths, the accuracy from different models becomes very similar. Although the models start at different R-square values, they all converge to an approximately $88 \%$ prediction accuracy (correlation coefficient of 0.94) with longer input data lengths. The PVAR model with threshold of 0.4 (in magenta) has the best performance when the input data length exceeds 3000 days (Figure 4.4 bottom panel). Although the AR model has the least prediction accuracy $\left(R^{2}=0.87\right)$ when the input data length is above 4000 days, the difference is only $2 \%$ lower from the best model (PVAR $0.4: R^{2}=0.89$ ). The AR model requires the least number of coefficients and offers high prediction accuracy. For the rest of the chapter, The AR model is adopted as the model to predict tidal weather. This experiment implies that the neighboring time series do not contain significantly more 
information than one single time series. They are highly correlated spatially (Figure 4.3), most likely because the 23 - day oscillation is present in all neighboring heights and latitudes.

Next, the accuracy of the AR model is tested for different lags. Figure 4.5 shows the prediction accuracy of the $\mathrm{AR}$ model as a function of lag. The $R^{2}$ for 1-day prediction is 0.7 with lag 1 , and 0.76 with lag 2 and increases to 0.87 for lag 23 . As mentioned earlier, the optimal lag should be determined by the data, as did in Figure 4.2, the data gives us an optimal lag of 23. Lower or higher lag has no physical meaning.

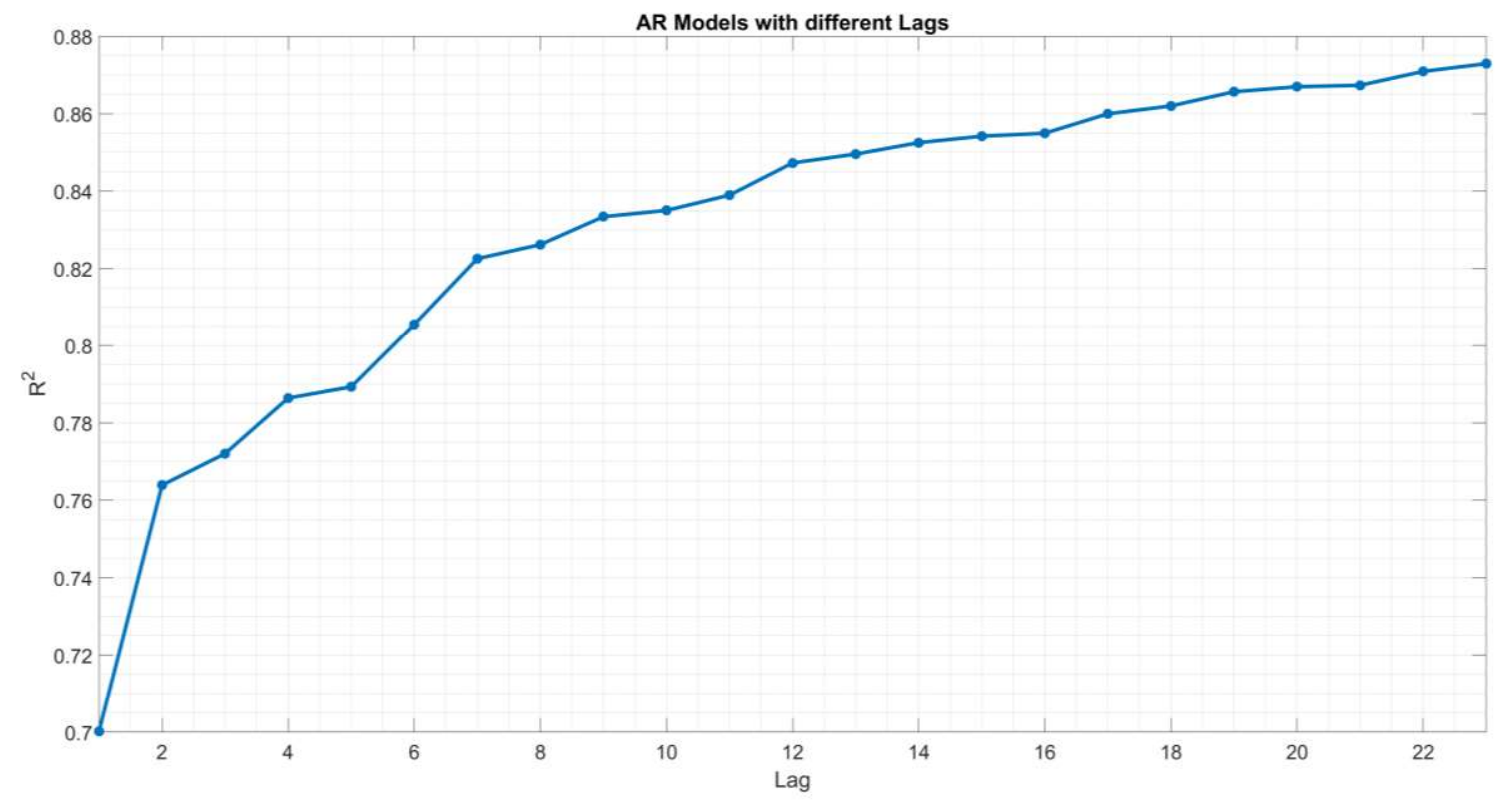

Figure 4.5. Prediction accuracy of the AR model as a function of lag.

In this section, $R^{2}$ is used to test the prediction accuracy of each models. In addition to $R^{2}$, anomaly correlation coefficient (ACC) also can use to assess the prediction accuracy. ACC is one of the most widely used measures in the numerical weather prediction. The anomaly correlation coefficient has the same basic equation as $R^{2}$ if the seasonality is excluded from the time series, except $R^{2}$ is correlation coefficient squared. There are different approaches on how to exclude the seasonality 
in the time series. In the ACC calculation, seasonality is excluded by averaging all January 1 , and so on. In the $R^{2}$ calculation, a low frequency band pass is used. As shown in Figure 4.6, the ACC squared values are very similar to the $R^{2}$. Hence, $R^{2}$ is used in the rest of the chapter to assess the prediction accuracy.

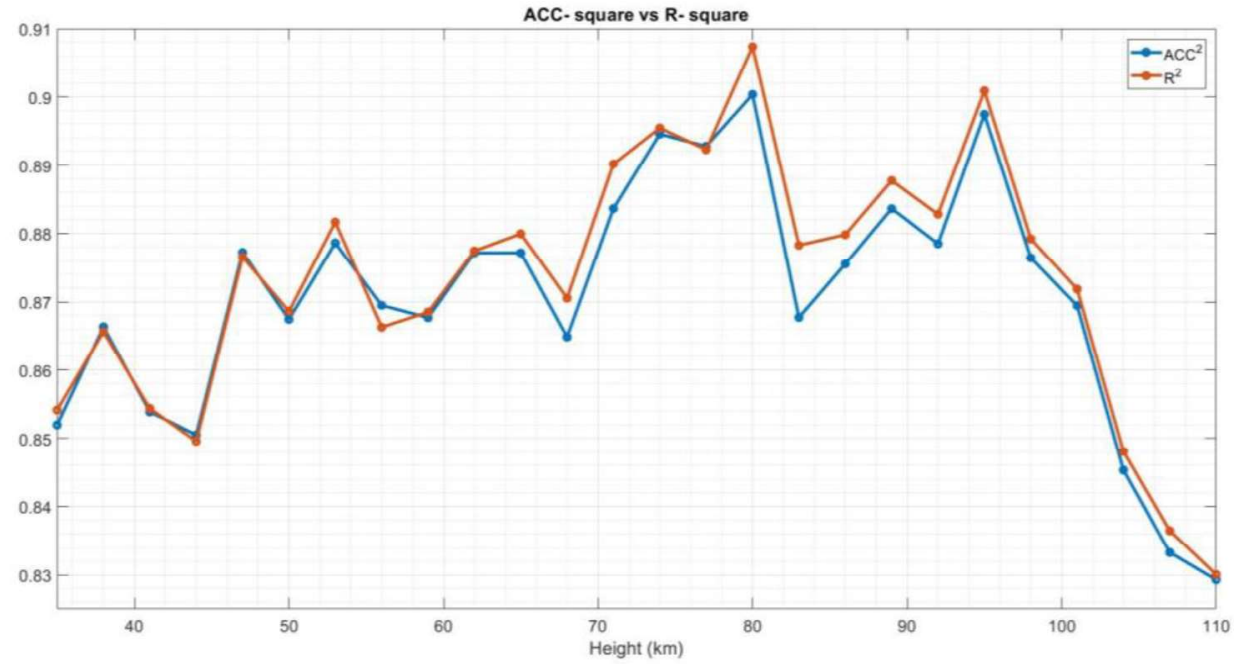

Figure 4.6. ACC squared and R squared values as a function of height for 1-day prediction in the AR model.

One important aspect of a statistical model is its robustness, e.g., do the coefficients need to be updated frequently? If the variability of the estimated model parameters is large, it is difficult to implement such model without frequently updating the coefficients. Here, in order to address such concerns, the prediction accuracy is compared for fixed coefficients and updated coefficients (on a daily basis) in terms of the $R^{2}$ values. Such experiments are carried out for 120,365, and 2000-day input data lengths for coefficient estimation as a function of height (Figure 4.7). For the 120-day input data length (black lines), the prediction accuracy ( $R^{2}$ values) is different for the models with fixed (solid black line) from those with variable coefficients (dashed black line), which implies that the updated coefficients are quite different from the fixed ones. With the 365-day input data length (blue lines), the $R^{2}$ values for the models with fixed (solid blue line) and variable (dashed blue line) coefficients 
are similar, but some discrepancy still occurs. With the 2000-day input data length (orange lines), the $R^{2}$ values for the fixed (solid line) and variable (dashed line) coefficients are close to overlapping for all heights, which implies that the coefficients from these two methods are very similar. This experiment shows that the coefficients are robust when a sufficient input data length is used to calculate these coefficients, and these coefficients do not need to be updated frequently. An input data length of 2000-days is most ideal: it provides relatively high predictive accuracy (Figure 4.4), and the coefficients are robust and do not require frequent updating (Figure 4.7).

The last test compares the prediction accuracy of the predicted period (June 30, 2008, to June 30, 2010), using the coefficients (2000-day input data length) from two periods: 2003 - 2008 and 1979 - 1984. Figure 4.8 shows the DW1 tidal weather anomaly from eCMAM at $100 \mathrm{~km}$ at the equator in 2009 (blue line), the predicted tidal weather using coefficients estimated from 1979 - 1984 (black line) and the predicted tidal weather using the coefficients estimated from $2003-2008$ (red line). It is clearly shown in Figure 4.8 that there are periodic oscillations in the short-term tidal variability, as shown by the auto-correlation (Figure 4.2), the dominant periodicity is about 23 days. The $R^{2}$ values between the predicted tidal weather and the original tidal weather time series for both cases are calculated and the $R^{2}$ values (0.874 vs. 0.872$)$ are so similar that the time series almost overlap. This experiment again proves that the coefficients calculated from the 2000 days are robust and do not require frequent update. The fixed AR coefficients calculated from the 2000 days prior to June 30, 2008 are adopted as the input data $(2003-2008)$ in this chapter.

\subsection{Tidal Weather Forecast}

Now, we are ready to predict tidal weather and examine the model prediction accuracy as a function of prediction day. The prediction procedure is as follows: tidal weather on January 1, 2009, and beyond is forecasted using equation 4.1, where the coefficients 


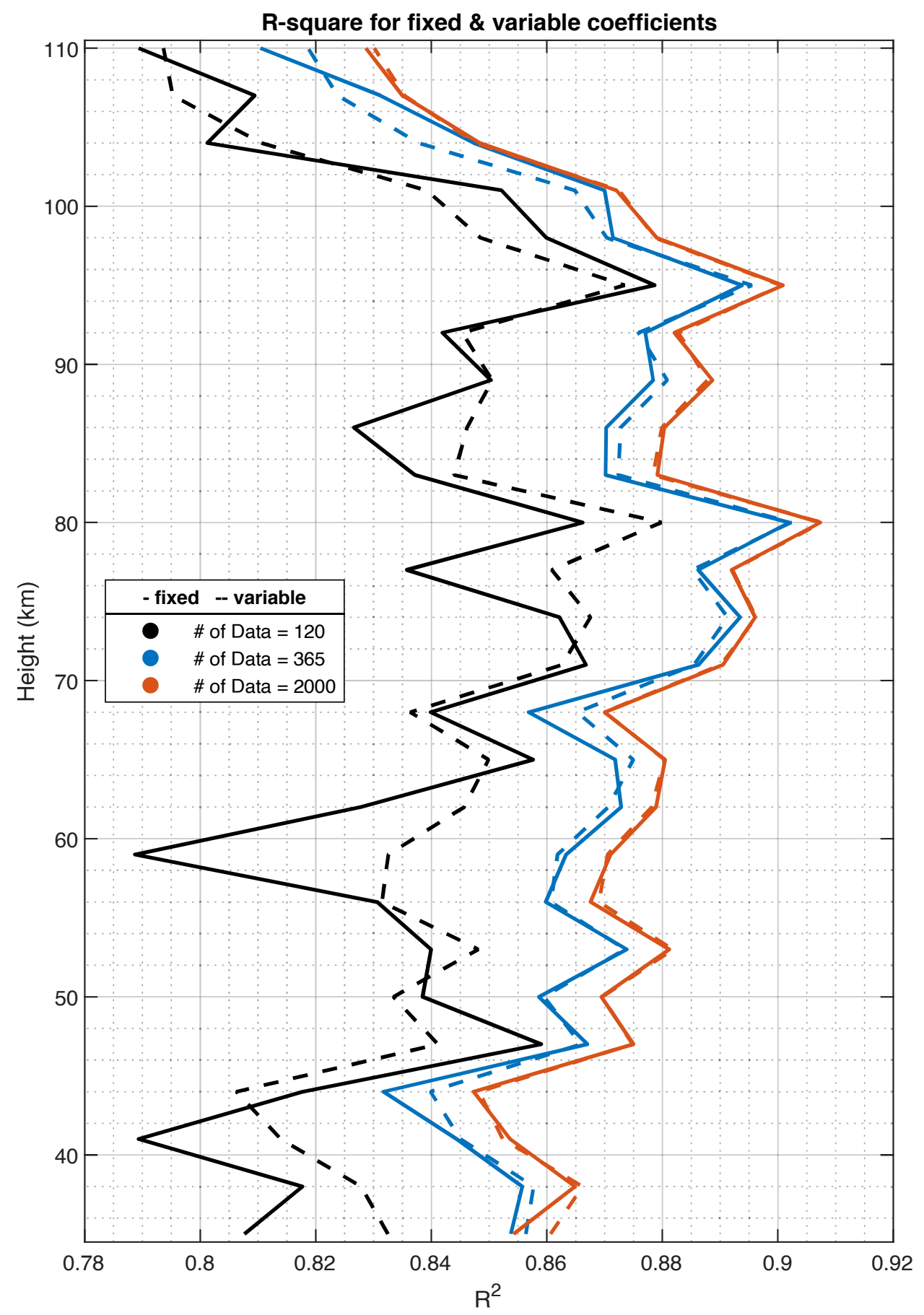

Figure 4.7. Prediction accuracy ( $R^{2}$ values) of fixed (solid) and variable (dashed) coefficients for different input data length (120-, 365-, and 2000-day) as a function of height in the AR model. 


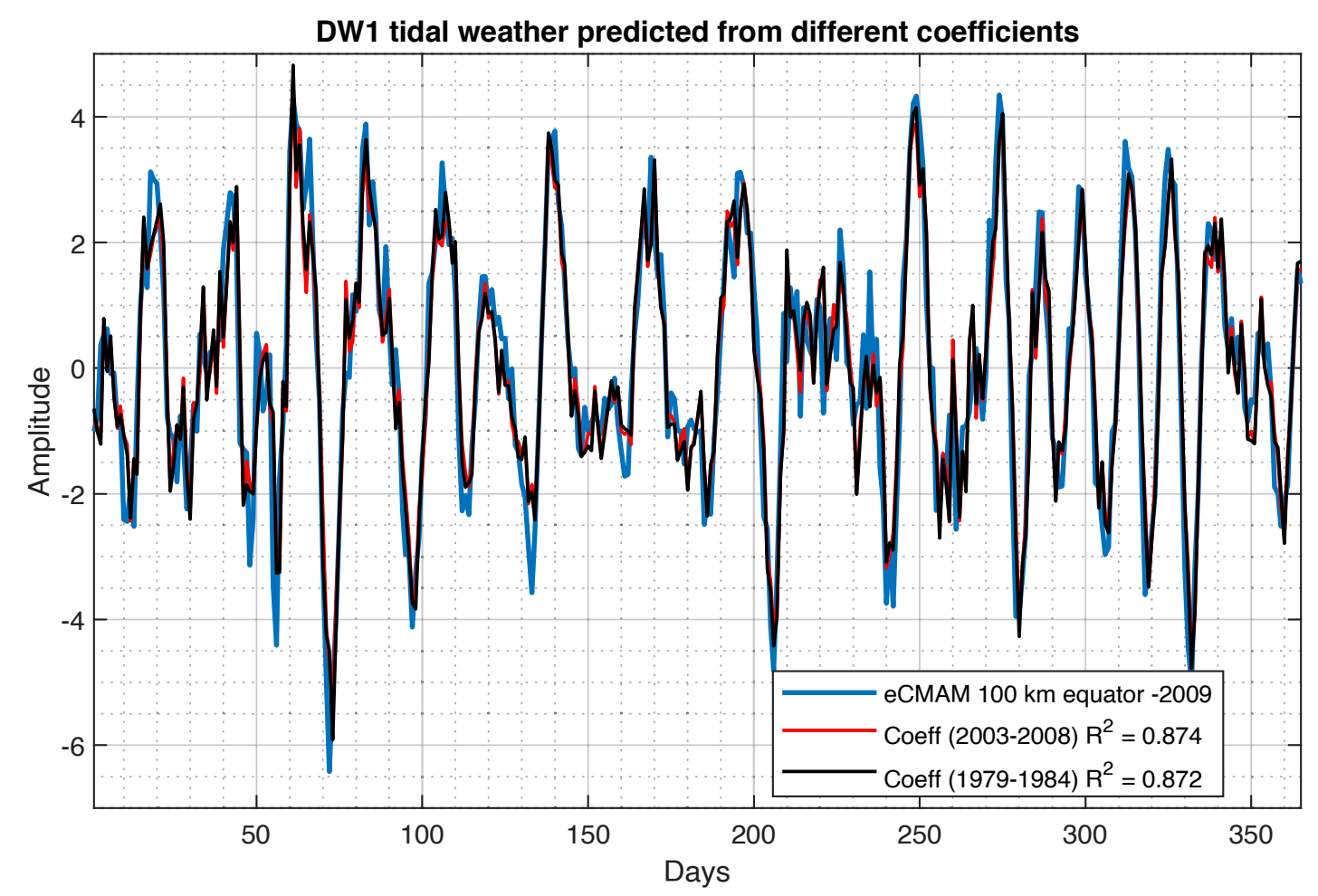

Figure 4.8. Predicted tidal weather of 2009 at $100 \mathrm{~km}$ and the Equator using coefficients calculated from 2000-day data in the 2003 - 2008 period (red line) and from 2000-day data in the 1979 - 1984 period (black line) and the original tidal weather time series (blue line).

are determined using the 2000 days (between 2003 - 2008) of tidal weather data in eCMAM. The 23 days of tidal weather data prior to January 1, 2009, are known and plugged in equation 4.1 to predict January 1, 2009, as the one-day prediction. January 2, 2009, is two-day prediction, and so on. January 2, 2009, at one-day prediction was calculated the same way, but using the 23-day tidal weather data prior to January 2, 2009. This process is repeated for the whole year. Figure 4.9 (left upper panel) gives an example of the eCMAM tidal weather for 2009 as a function of day and heights, and Figure 4.9 (left bottom panel) gives the predicted tidal weather at one-day with the AR model for the same time span. It is observed that the one-day prediction is 

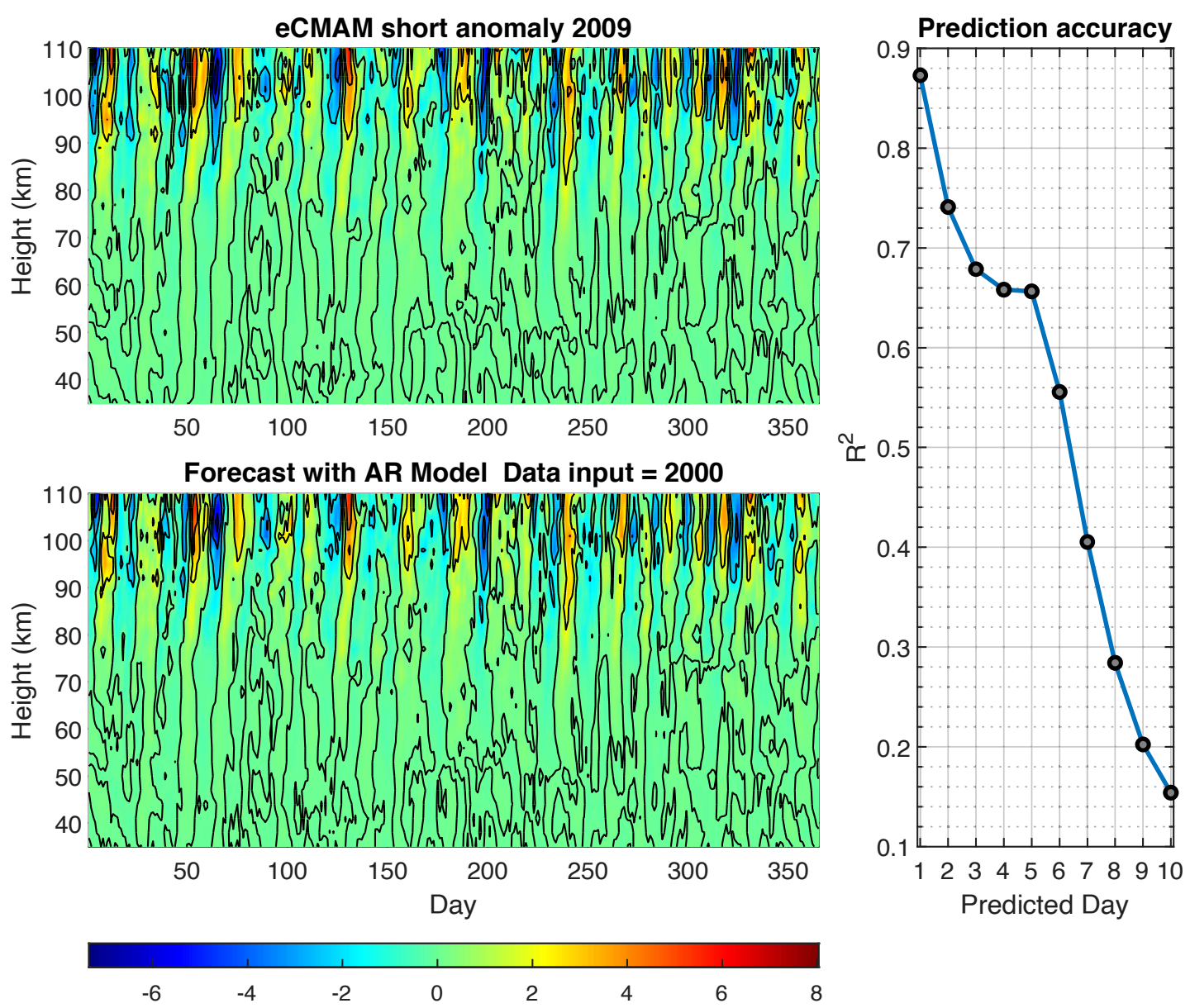

Figure 4.9. Top left: Tidal weather anomaly in 2009 from the eCMAM.Bottom left: Predicted tidal weather anomaly in 2009 with the AR model. Right: Prediction accuracy (average $R^{2}$ values over heights) as a function of future predicted days.

almost identical to the original eCMAM tidal weather variability. $R^{2}$ values are calculated between the original eCMAM tidal weather variability and the AR model predicted tidal weather at each height. Figure 4.9 (right panel) shows the averaged $R^{2}$ values over all heights as a function of the number of predicted days. $R^{2}$ decreases as the number of predicted day increases. The prediction accuracy for one day is the highest and $\sim 87 \%$ (correlation coefficient $\sim 0.93$ ). It gradually decreases to $\sim 75 \%$ (correlation coefficient $\sim 0.87$ ) for two days, $68 \%$ (correlation coefficient 0.82 ) for three days, and $65 \%$ (correlation coefficient 0.81 ) for four- and five-day prediction. 


\subsection{Comparison with SABER}

Next step is to validate the coefficients used in the AR model obtained from the eCMAM input data with coefficients calculated from SABER tidal weather data. In other words, another AR model is built based on SABER tidal weather data (2003 - 2008, 2000 - day data input) and compare these 23 coefficients with the coefficients obtained from the eCMAM. The AR model also uses a lag of 23 for SABER data. The coefficients estimated from both models (eCMAM and SABER) are plotted in Figure 4.10 at 35, 65, and $100 \mathrm{~km}$, respectively. Orange lines are coefficients estimated from SABER, and the blue lines are from eCMAM. It can be seen that the two sets of coefficients are very similar to each other at all three heights. The smaller coefficients at days 2, 3, and 4 are due to the five-day averaging window performed on the original time series. The weight of the coefficients is more for the first (day 1) and last day (day 5) of the averaging window. This is also true for the next averaging window at days 7,8 , and 9 , and so on. The coefficients decrease gradually at days $1,6,11,16,21$, which implies that yesterday's tidal weather is more important to today's forecast than tidal weather happened six days ago.

The AR model is tested for other average windows of different sizes (three to seven days). Figure 4.11 shows the relative distribution of the 23 coefficients for different averaging windows. The size of the window does not affect the statistical properties (e.g. auto-correlation and spatial correlation), but rather the relative distribution of the 23 coefficients, in other words, the relative weight of the coefficients.

Then the AR models with SABER and eCMAM coefficients are applied to predict SABER tidal weather during the prediction period (June 2008 - June 2010). Figure 4.12 shows the original SABER tidal weather anomaly for 2009 as a function of day and height at the equator (left upper panel), the one-day prediction from the AR model with SABER coefficients (left middle panel), and one-day prediction from the AR model with eCMAM coefficients (left bottom panel). The similarity of these three 
AR Model coefficients $100 \mathrm{~km}$ at the equator

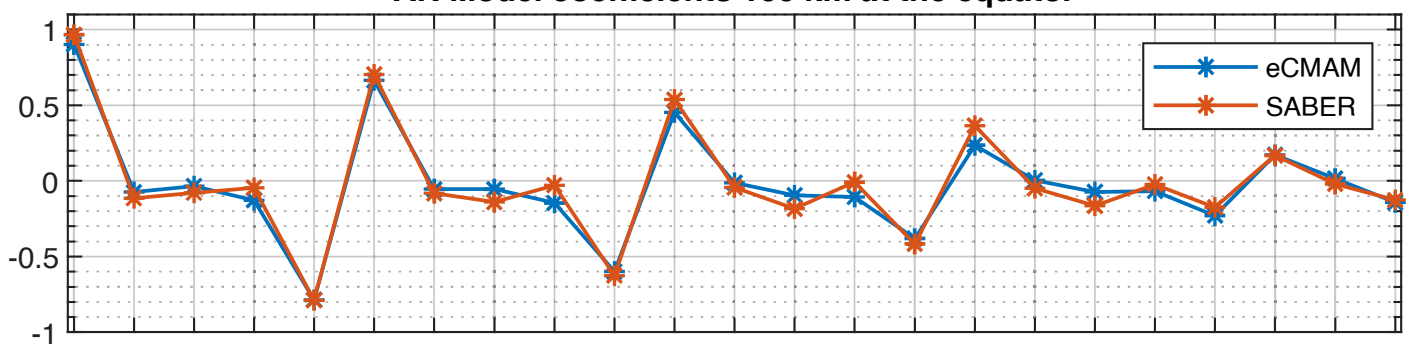

AR Model coefficients $65 \mathrm{~km}$ at the equator

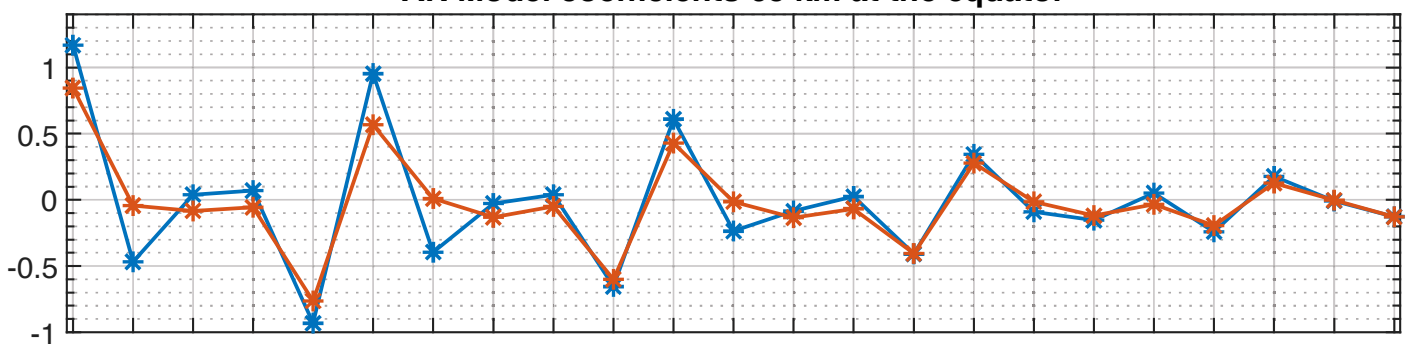

AR Model coefficients $35 \mathrm{~km}$ at the equator

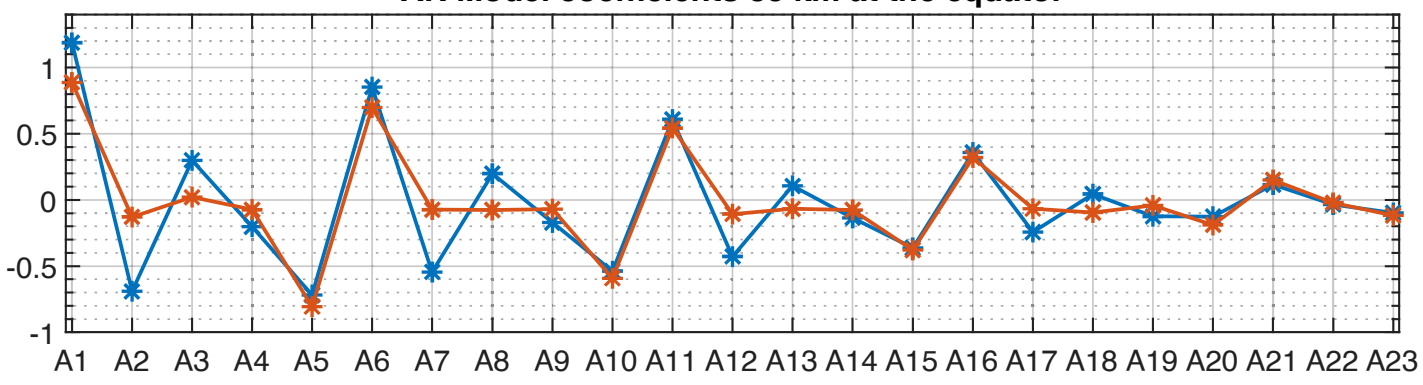

Figure 4.10. The 23 coefficients in the AR models from both eCMAM (blue lines) and SABER (orange lines) at 100 (top), 65 (middle), and $35 \mathrm{~km}$ (bottom).

figures indicates that the observed tidal weather from SABER can be effectively simulated and predicted by the AR model with coefficients obtained from the eCMAM. The height averaged $R^{2}$ values for predicted days 1 to day 5 are calculated for the AR model with eCMAM coefficients (red line) and with SABER coefficients (blue line) are plotted in Figure 4.12 right panel. The two lines not only have a similar dependence on day, but also are very close in value. The prediction accuracy is slightly higher for the AR model with SABER coefficients. The day 1 accuracy is about $84 \%$ (85\%) for AR model with eCMAM (SABER) coefficients. This again demonstrates the robustness of the coefficients obtained from the eCMAM in the AR model and validates their use for tidal weather prediction. 


\section{AR Model coefficients 3- day averaging window}
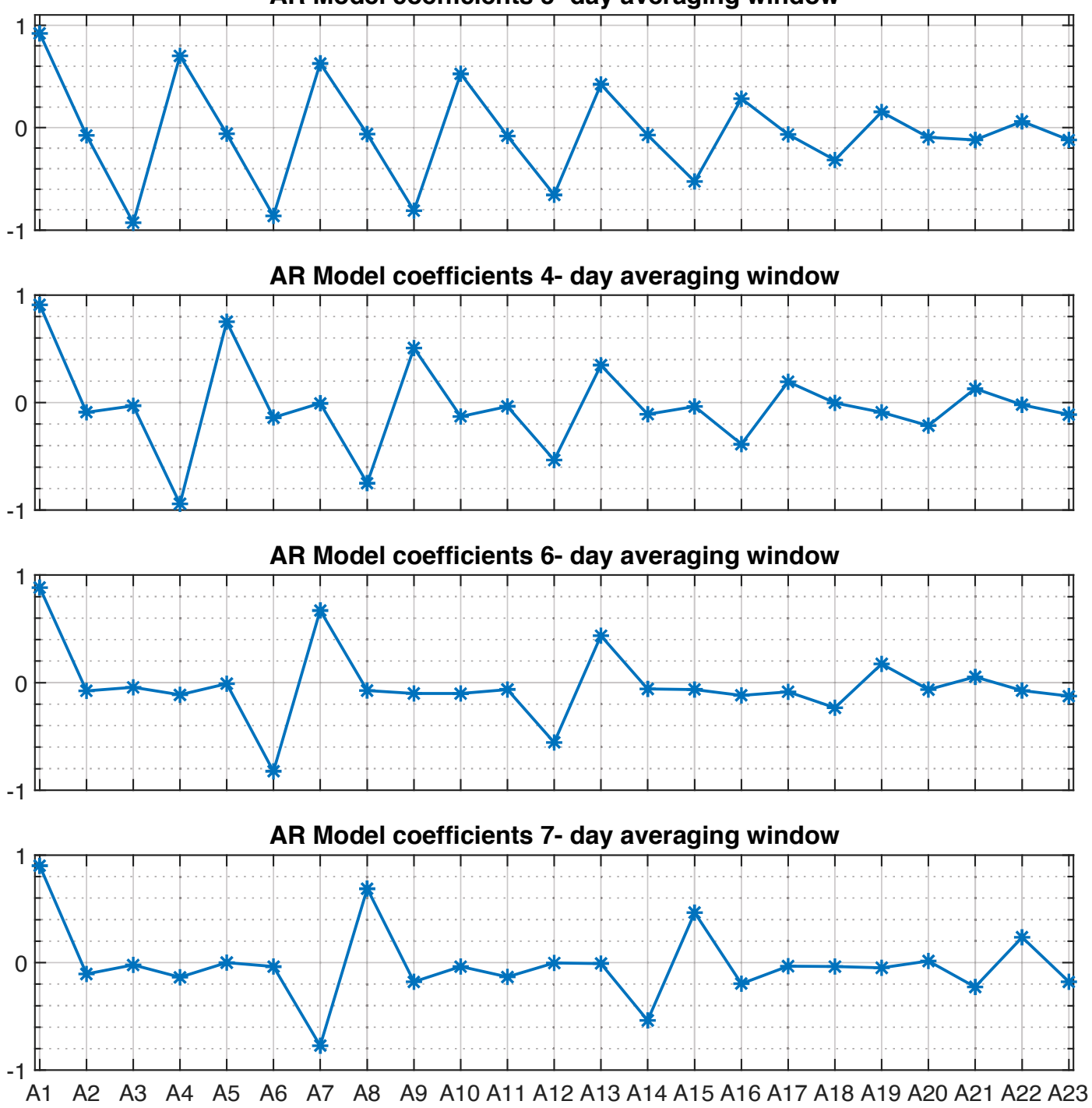

Figure 4.11. The 23 coefficients in the AR models for 3-day (first), 4- day (second), 6- day (third), and 7- day averaging window in eCMAM at $100 \mathrm{~km}$. 

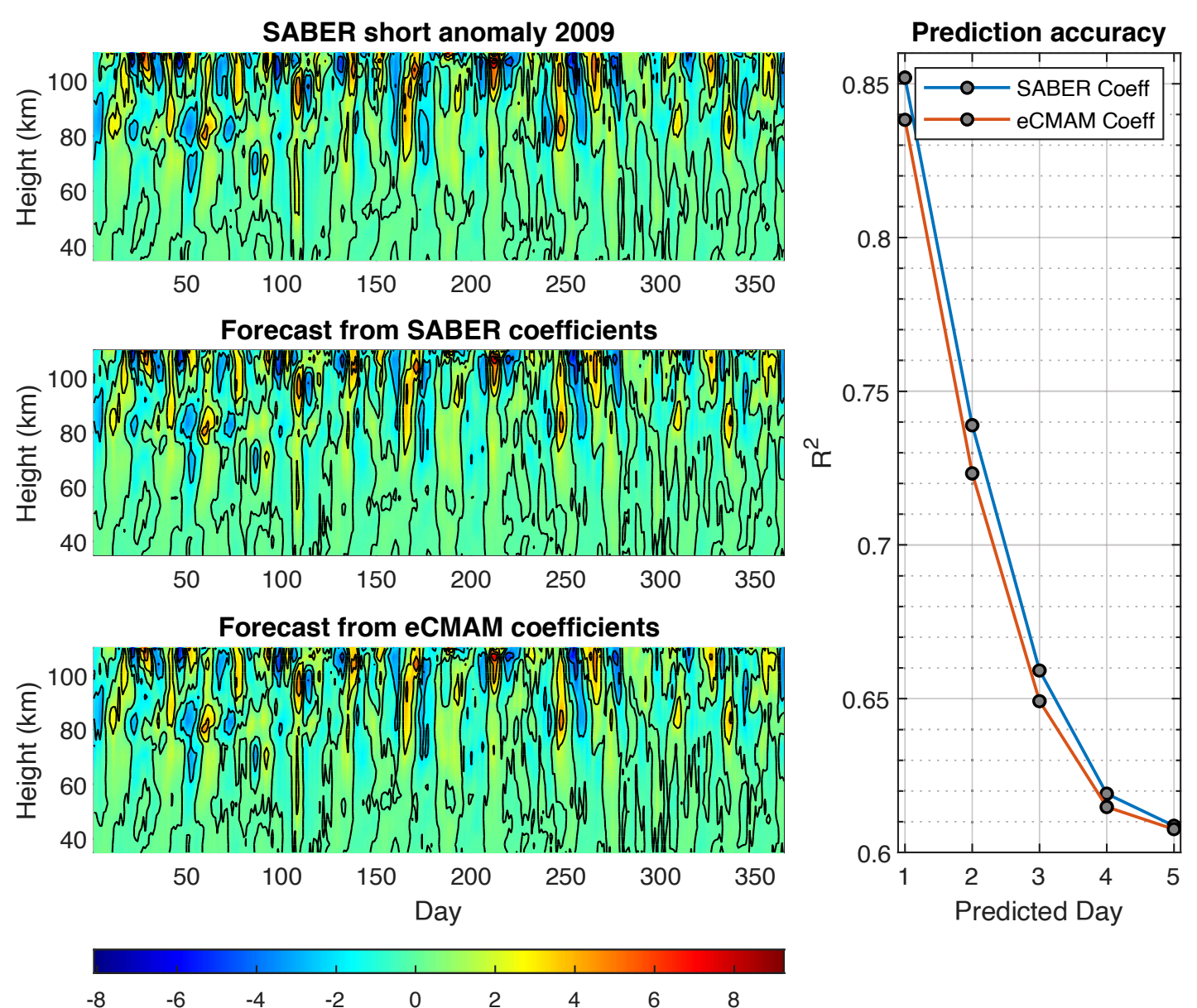

Figure 4.12. Tidal weather anomaly in 2009 from SABER (top left), predicted tidal weather anomaly in 2009 from AR model with SABER coefficients (middle left), and predicted tidal weather anomaly in 2009 from AR model with eCMAM coefficients (bottom left). (Right) Prediction accuracy (average $R^{2}$ values over heights) as a function of future days for AR models with eCMAM (orange line) and SABER (blue line) coefficients. 


\subsection{Discussion and Conclusions}

The models and observations have shown significant day-to-day variability of the tides for two decades. However, up to today, we have very little knowledge of it, e.g., how these variabilities evolve with time and space, is there any coherent temporal variability or is it stochastic, and what are the physical mechanisms behind. To better understand a complicated system as tidal weather (short-term tidal variability), we first need to understand it statistically. How does it change with time, is there any self memory, or totally stochastic? How does it change with space, are (or to what extent) they related to their neighboring latitudes/heights, or are they totally stochastic? These are the fundamental questions that we have to answer before we can go any further with our understanding of tidal weather. To our knowledge, this investigation is making the first step to understand the complex system of short-term tidal variability.

DW1 tidal variability was separated into tidal weather and long-term tidal variability using a Fourier bandpass filter. Wavelet analysis reveals that there is a rich spectrum of short-term variability between periods of 8 - 30 days. The autocorrelation (Figure 4.2) of the tidal weather shows that there is a quasi-23-day periodicity in both eCMAM and SABER. The quasi-23-day oscillation is also evident in the tidal weather anomaly time series in Figure 4.8. The 23-day oscillation is the dominant period in DW1 tidal weather, and it may be one dominant wave with the 23-day period, or a wave packet containing the superposition of more than one wave. Identifying this dominant periodicity in DW1 tidal weather is a big step forward on our understanding of short-term tidal variability. Tidal weather is spatially strongly correlated with its neighboring latitudes and heights in both eCMAM and SABER. The height range of strong correlations may be related to the vertical wavelength of the tide. These statistical properties are utilized to build a simple AR model and two more complex models, e.g., VAR and PVAR. The simple AR model, which takes into account only 
the auto-correlation, has 23 coefficients at each location (latitude and height) and can predict tidal weather to an accuracy of $89 \%$ at 1 - day. The VAR and PVAR models, which take into account not only the auto-correlation but also the spatial correlations between the neighboring altitudes and latitudes, slightly outperform the simple AR model but are much more computationally expensive with many more coefficients. The fact that the PVAR and VAR do not significantly improve forecast accuracy implies that the neighboring time series do not contain significantly more information than one time series. They are highly correlated spatially, most likely due to the common 23-day oscillation.

It can be seen that at least 24 data values ( $y$ 's) are needed to form the equation 4.1 in the first place. However, with just 24 values, $A_{i}$ and $C_{1}$ can take an infinite number of possible values to form equality. In other words, more constraints are needed to set up a linear system and compute the parameters $A_{i}$ and $C_{1}$. For 24 parameters, 24 equations are needed to get a stable solution of the linear system. In total, $24+23=47$ data points are needed ( $y$ 's) to feed this equation and estimate the parameters $A_{i}$ and $C_{1}$. Fewer points result in less robust coefficients. Figure 4.7 and 4.8 shows that with 2000 days of data, the coefficients are robust and do not need to be updated continuously. Hence, in the simple AR model, 2000 days of data have been used to calculate the 23 coefficients. In order to make the actual prediction, 23 days of prior data are needed.

A similar AR model was built based on tidal weather data from SABER. The coefficients of the AR model from SABER data agree very well with those from the eCMAM. The AR model with coefficients estimated from eCMAM performs nearly as well as that built on SABER. With the 23 coefficients and the prior 23-days of tidal weather data, the next day's tidal weather can be predicted at near $90 \%$ accuracy.

This study shows that there is a quasi-23-day oscillation in DW1 tidal weather. By taking advantage of this periodicity, future tidal weather can be simulated and 
forecasted with high accuracy. Eckermann et al. (1997) and Miyoshi and Fujiwara (2003) found a similar period of variability in their DW1 tidal weather data. Both studies suggested that this period of fluctuations in DW1 might be induced by similar periodicities in the tropospheric convective activity (Hartmann et al., 1992). However, no detailed study has been conducted to prove the linkage. Extensive future work is needed to interpret the physical mechanisms of this quasi-23-day oscillation observed in DW1 tidal weather. Possible sources include wave-mean flow interaction, wavewave interaction, and heating, or a combination of them. 


\section{CHAPTER 5}

\section{CHARACTERISTICS OF TOTAL DW1 VARIABILITY IN THE MESOSPHERE AND LOWER THERMOSPHERE}

In this chapter, the DW1 total tidal temporal variability is analyzed on various time scales and their relative importance using the data from eCMAM30 and SABER. SABER version 2.0 data from January 2003 to December 2016 and from 60 to $95 \mathrm{~km}$ is used in this study. eCMAM data ranges from 1979 to 2010 and from 60 to $110 \mathrm{~km}$. Both the eCMAM30 and SABER temperature data are interpolated to have the same resolution in geometric height $(3 \mathrm{~km})$ and latitude $\left(5^{\circ}\right)$. The daily tidal amplitude is averaged with a five-day moving window and shifted by one day for both datasets.

This chapter is organized as follows. Section 5.1 describes the total tidal variability of DW1 and its wavelet analysis. Section 5.2 presents a multiple linear regression model fitted to both the model and observation, accounting for the solar cycle, ENSO, QBO, and seasonal harmonics. Section 5.3 compares DW1 tidal temperature climatology between eCMAM and SABER as a function of height and latitude. Interannual and seasonal coefficients obtained from the multiple regression model are presented in Sections 5.4 and 5.5, respectively. Section 5.6 describes the long-term variability presented in the short-term tidal variability. Relative importance of short-term, seasonal, and interannual variability is presented in Section 5.7. Section 5.8 predicts the total tidal variability based on the multiple linear regression model and the tidal 
weather forecast model from Chapter 4. This chapter closes with the discussion and conclusions in Section 5.9.

\subsection{Total Tidal Variability of DW1}

This chapter studies the total temporal variability in the DW1 temperature amplitudes from the eCMAM and SABER. We first present the total time series of DW1 temperature amplitudes as a function of latitude and time (from January 2003 to June 2010) at $95 \mathrm{~km}$ for the eCMAM (top) and SABER (bottom) in Figure 5.1. The white dashed lines in the eCMAM panel show the same latitude range as SABER. The DW1 amplitude in eCMAM exhibits the classical DW1 feature with maximum over the equatorial region and secondary maxima symmetrically located at $\sim 35^{\circ} \mathrm{N} / \mathrm{S}$. The semiannual variation is evident with maxima in equinoxes and minima in solstices. The DW1 amplitude also exhibits strong interannual variation with stronger amplitude observed in 2004, 2006, and 2008, which is likely linked to the stratospheric QBO (Gan et al., 2014). The equatorial DW1 maximum amplitude in eCMAM is larger than in SABER. The classical feature of the DW1 amplitude (maximum over the equator and secondary maxima in the subtropics) is less clear in SABER. This may be due to increasing aliasing from the migrating semidiurnal tide poleward of $20^{\circ}$.

The periods that dominate the temporal variation of the DW1 amplitude are examined by performing a wavelet analysis (Torrence and Compo, 1998). The wavelet analysis is performed on the total time series of DW1 $\mathrm{T}$ amplitudes at $95 \mathrm{~km}$ and the equator for both datasets. The wavelet power spectrum used here is the Morlet wavelet. Figure 5.2 shows the results of the wavelet analysis for the eCMAM (top) and SABER (bottom). The cross-hatched regions indicate the cone of influence (within the cone of influence, the results are not significant) and the solid black lines represent the $95 \%$ confidence level. Both wavelet analysis from eCMAM and SABER illustrates 
a wide range of temporal variability, ranging from solar cycle scales to a few days.
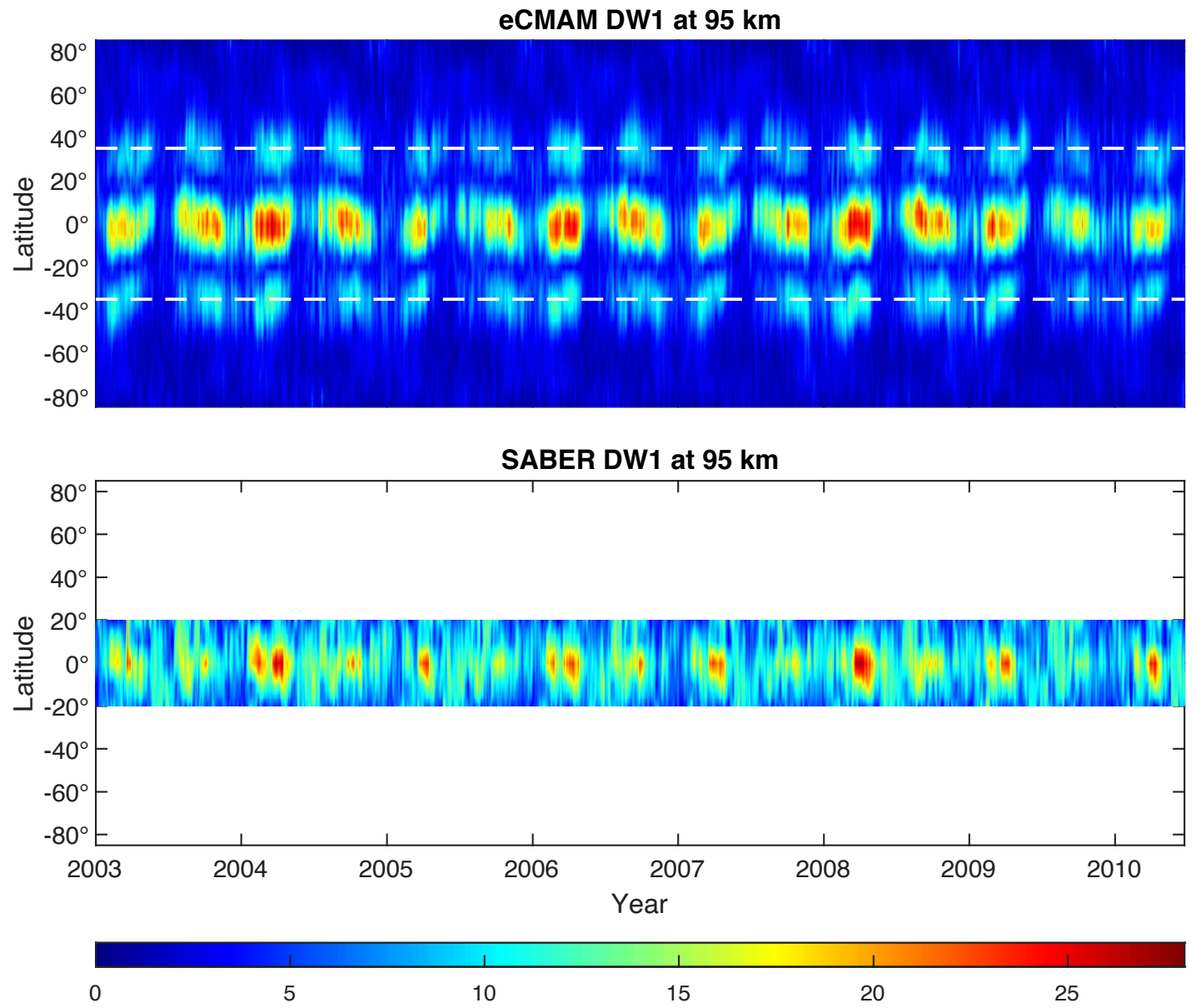

Figure 5.1. The DW1 temperature amplitude $(\mathrm{K})$ as a function of latitude and time (from January 2003 to June 2010) at $95 \mathrm{~km}$ from the eCMAM (top) and SABER (bottom).

Short-term tidal variability on a scale of 5 to 30-day is likely related to modulations from transit planetary waves, short-time variability in the background wind where the tide propagates through and/or tidal source variability. We will investigate the physical mechanisms of short-term tidal variability in Chapter 6 . There is also an intra-seasonal variation of 30-90 days presented in both datasets. The causes for these variation periods are possibly related to the Madden Julian Oscillation (MJO) (Yang et al., 2018). The well-known semi-annual variation (180 days) of the DW1 amplitude is evident in both datasets. Interannual variation such as QBO (25-30 
months), ENSO and/or solar variability is located within the cone of influence due to the limited time span (7.5 years) of the data used for the wavelet analysis.
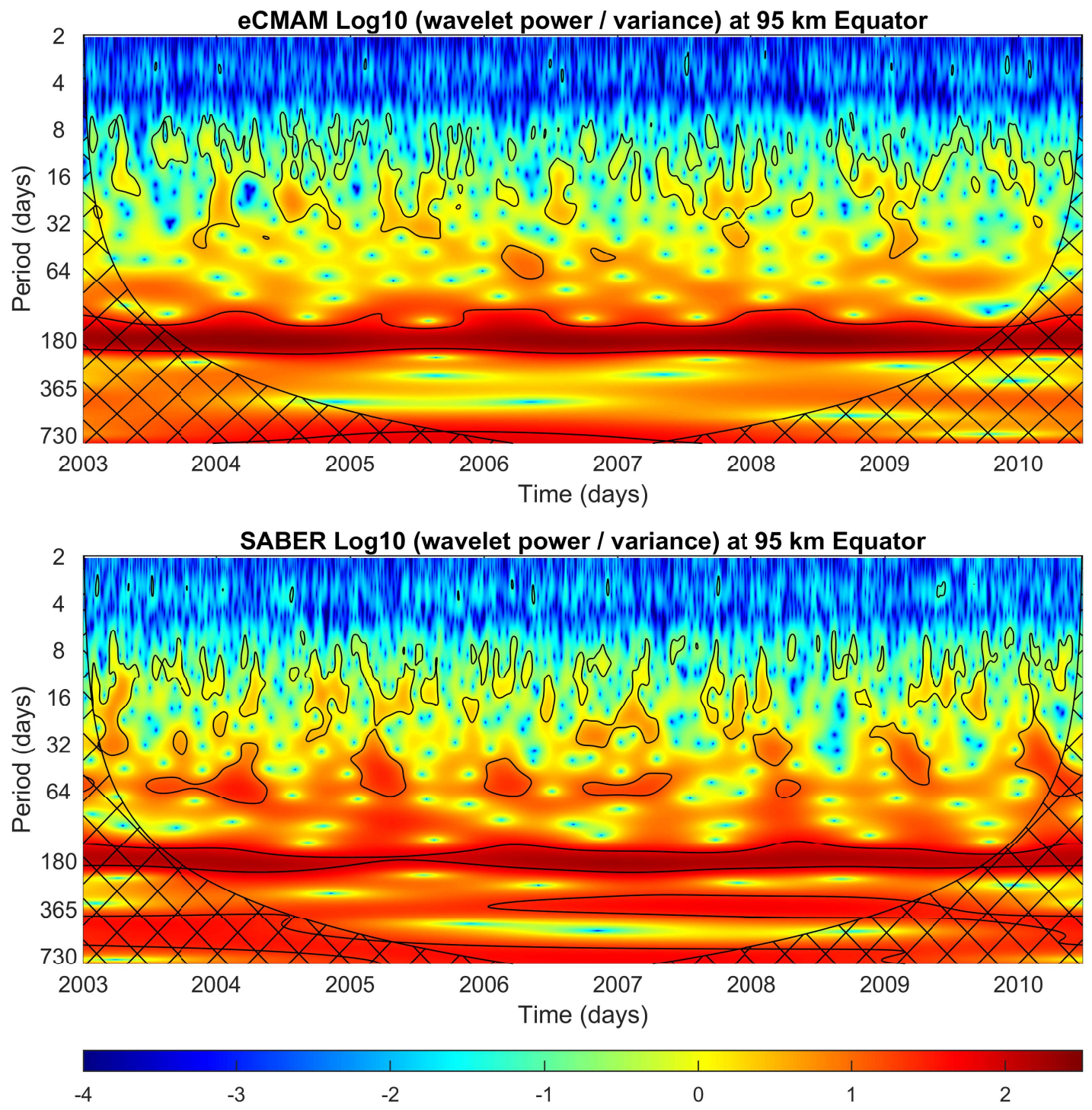

Figure 5.2. Wavelet analysis of the DW1 $\mathrm{T}$ amplitude at $95 \mathrm{~km}$ and the equator for the eCMAM (top) and SABER (bottom). The cross-hatched regions indicate the cone of influence (within the cone of influence, the results are not significant) and the solid black lines represent the $95 \%$ confidence level. 


\subsection{Multi-linear Regression Model}

To examine the total tidal variability modulated by various temporal scales in more detail, we employ a multi-linear regression model to fit both eCMAM and SABER data. Multi-linear regression is a statistical technique that models the relationship between two or more independent variables (predictors) and a dependent variable (predictand) by fitting a linear equation to the data. Performing a regression analysis

allows us to identify which variables have impact on a topic of interest and which variables can be ignored. The multi-linear regression model used in this dissertation takes the following form:

$$
\operatorname{tide}_{i}(t)=t i d e_{i o}+\alpha_{i} t+\beta_{i} E(t)+\chi_{i} Q(t)+\delta_{i} S(t)+\sum_{k=1}^{n} a_{i, k} \cos \left(\omega_{k} t+b_{i, k}\right)+\epsilon_{i}(t)
$$

where the subscript $i$ denotes a particular latitude and height such that $\operatorname{tide}_{i}(t)$ is the atmospheric tide as a function of time $t$ at a given location. tide $_{i o}$ represents the mean tidal amplitude. The coefficient $\alpha_{i}$ represents the strength of the linear trend and $\beta_{i}$ is the strength of ENSO and $E(t)$ is the multivariate ENSO index obtained from https://www.esrl.noaa.gov/psd/enso/mei/. $\chi_{i}$ represents the strength of $\mathrm{QBO}$ and here we use the $\mathrm{QBO}$ index $Q(t)$ retrieved from https: //www.cpc.ncep.noaa.gov/data/indices/qbo.u30.index. $\delta_{i}$ is the coefficient of the solar variability and $S(t)$ is the solar radio flux at $10.7 \mathrm{~cm}$ taken from https: //lasp.colorado.edu/lisird/data/penticton_radio_flux/. The cosine terms represent the seasonal variations, $n$ is the number of harmonics (here $n=4$ ) and $\omega_{k}$ is frequency: $n=1,2,3$, and 4 correspond to the 12, 6, 4, and 3- month harmonic, respectively. $a_{i, k}$ and $b_{i, k}$ are the corresponding amplitude and phase of the seasonal harmonics. All of the above terms described are considered deterministic responses. The residue $\epsilon_{i}(t)$ term includes the short-term tidal variability ( $<30$ days), and the long-term variability that is not resolved by the deterministic terms in the regression model. 
Figure 5.3 shows the normalized indices for ENSO (top), QBO (middle), and Solar Flux (bottom) used in the regression model from January 1979 to December 2016. These indices were normalized by dividing their maximum absolute value so that the coefficients obtained from the regression model have the same unit $(\mathrm{K})$ as the tide (predictor), and can be directly compared with each other. The multivariate ENSO index (MEI) is computed as the first unrotated principal component of six variables over the tropical pacific. The six variables are sea level pressure, zonal and meridional components of the surface wind, sea surface temperature, surface air temperature and total cloudiness fraction of the sky. The standardized departure $(y$-axis) shows the standard deviations from the mean. Positive values of the MEI represent the warm phase of ENSO - El Niño years, and negative values represent the cold phase - La Niña years (Wolter and Timlin, 1998, 2011).

The QBO is a quasi-periodic oscillation of the equatorial zonal wind between easterlies (westward) and westerlies (eastward) in the tropical stratosphere with a mean period of 28 to 29 months. For the QBO index, the Singapore wind anomaly at $30 \mathrm{mbar}$ is used. Positive values denote westerly QBO and negative values denote easterly QBO. ENSO and QBO values are available as monthly indices, hence are interpolated to calculate the daily values for the regression model. There is no shortterm variability included in these indices.

The bottom panel of Figure 5.3 shows the F10.7 solar radio flux. The F10.7 index is a measure of the noise level generated by the sun at a wavelength of $10.7 \mathrm{~cm}$ at the Earth's orbit. The $10.7 \mathrm{~cm}$ solar radio flux is one of the most widely used indices for solar activity. It is expressed in solar flux units (sfu), where $1 \mathrm{sfu}=10^{-22} \mathrm{~W} \mathrm{~m}^{-2} \mathrm{~Hz}^{-1}$ and F10.7 can vary from below 50 sfu to above 300 sfu over the course of a solar cycle (Tapping, 2013). The global daily value of this index is measured at local noon at the Penticton Radio Observatory in Canada. The solar radio flux at $10.7 \mathrm{~cm}$ correlates 

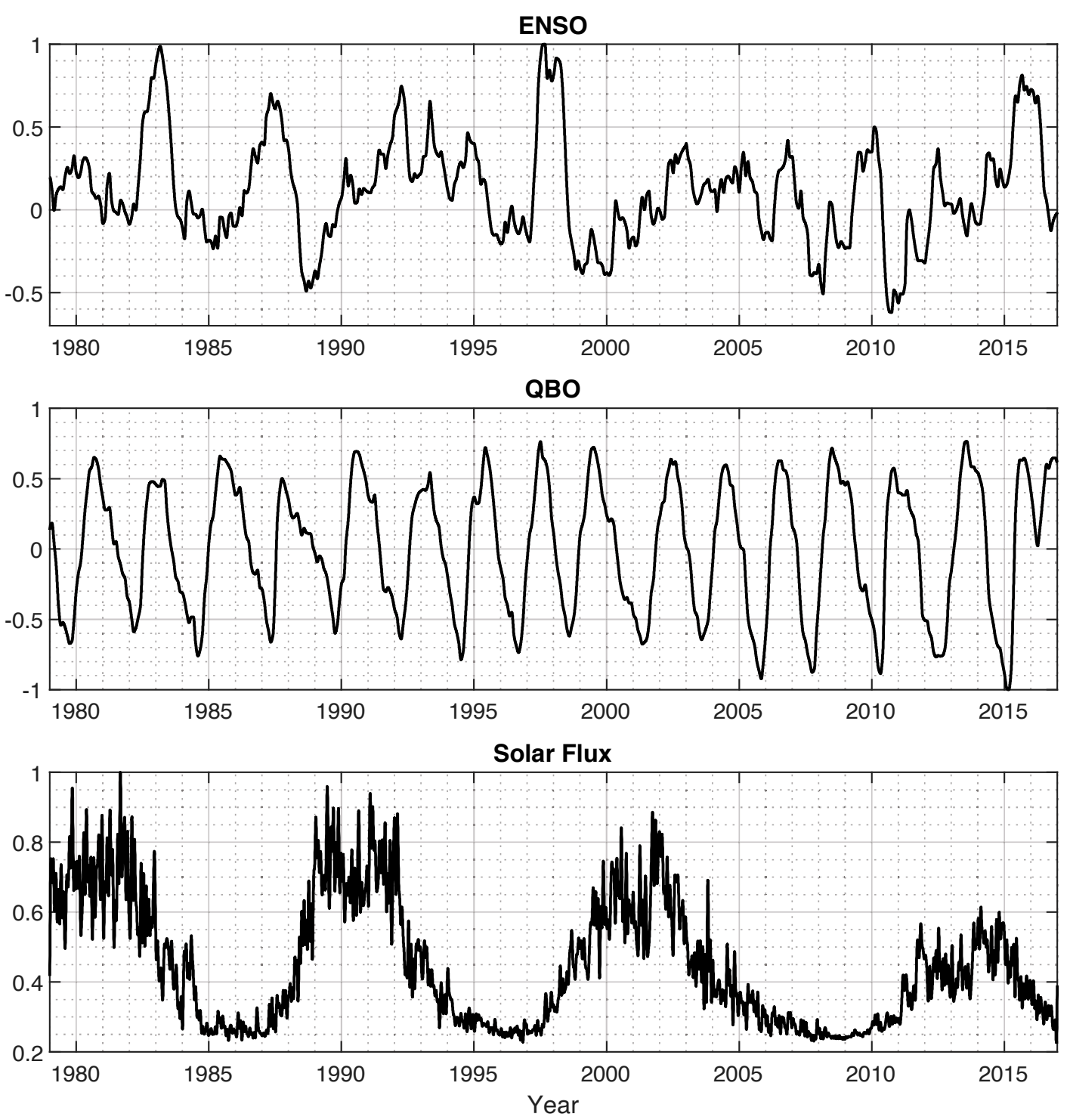

Figure 5.3. Normalized indices used in the multi-linear regression fitting: ENSO (top), QBO (middle), and solar flux (bottom).

well with the sunspot number and total solar irradiance. Ultraviolet emissions that affect the upper atmosphere and stratospheric ozone also correlate with the F10.7 index. During the data range of interest, solar activity reached its minimum around the year 1986, 1996, and 2009. The largest F10.7 index after 2000 occured in October 2003, which is known as the Halloween Storms. Since the F10.7 index is given as daily values, to keep all the short-term tidal variability in the residue term of the regression 
model, the F10.7 index used there is filtered using a Fourier low-pass filter to only keep variability period longer than 30 days.

\subsection{Climatological Mean of DW1}

In this section, we present tide $_{i o}$, the climatological mean of the DW1 temperature amplitude, in the regression model. Figure 5.4 shows the climatological mean of the DW1 T amplitude over the whole time range (eCMAM: January 1979 - June 2010 and SABER: January 2003 - December 2016) as a function of latitude and height: eCMAM (left) and SABER (right). The contour intervals are $4 \mathrm{~K}$. The mean structure of DW1 in eCMAM is characterized by an equatorial maximum and two
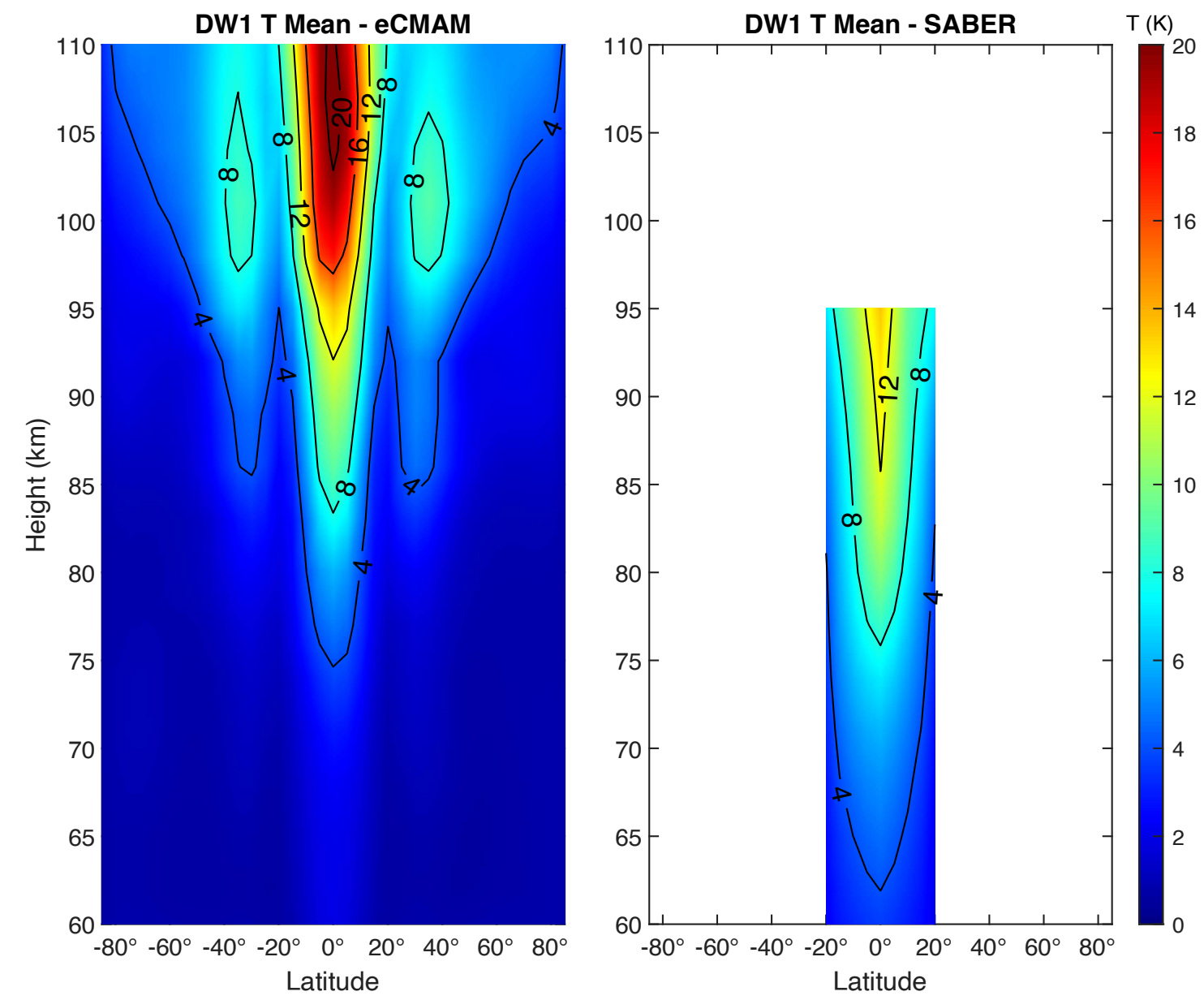

Figure 5.4. Climatological mean of the DW1 T amplitude (K) in eCMAM (left) and SABER (right). The contour interval is $4 \mathrm{~K}$. 
secondary maxima located symmetrically in the mid-latitudes (near $\pm 35^{\circ}$ ) of each hemisphere above $60 \mathrm{~km}$. The equatorial maximum is located between 100 and $110 \mathrm{~km}$ in eCMAM with amplitudes of 18 to $20 \mathrm{~K}$. The secondary maxima in the mid-latitudes have amplitudes of $8 \mathrm{~K}$ in eCMAM. SABER also shows a maximum over the equator, but with weaker amplitude (12-14 K) than the eCMAM.

\subsection{Interannual Variations of DW1}

In this section, we present the coefficients for variability greater than 12 months, e.g., the interannual variability, in the regression model. Figure 5.5 shows the coefficients of solar flux ( $\delta_{i}$, top), ENSO $\left(\beta_{i}\right.$, middle) and $\mathrm{QBO}\left(\chi_{i}\right.$, bottom $)$ in the regression model as a function of height and latitude for eCMAM (left) and SABER (right). We used normalized indices in the regression model, hence the unit of the colorbars in Figure 5.5 are in Kelvin. Multiplying the coefficients with indices (Figure 5.3) gives the corresponding temperature change related to each interannual variability. The latitudinal structures of all three coefficients follow the latitudinal structures of the tide in both the model and observation, implying that the response to the interannual variation is the strongest where the tide maximizes. For the solar effect on tidal amplitude (Figure 5.5 top), both eCMAM (top left) and SABER (top right) show positive correlations with the solar flux, which indicates that tidal amplitude is stronger during solar maximum than the solar minimum. The maximum response is located over the equator for both datasets but with eCMAM at a higher altitude (95-110 km) than SABER $(\sim 80 \mathrm{~km})$. The eCMAM presents secondary maxima in $30^{\circ}-40^{\circ} \mathrm{N} / \mathrm{S}$ as well, which corresponds to the secondary maxima in the DW1 amplitude of the same region. The eCMAM solar cycle coefficients are about $2-4 \mathrm{~K}$ above $100 \mathrm{~km}$. Below $90 \mathrm{~km}$, eCMAM shows negligible solar response while SABER shows maximum values of $\sim 0.5 \mathrm{~K}$. Since the normalized solar indices change between 0.2 and 1 between solar minimum and maximum, the eCMAM solar response during 
solar minimum is about $0.4-0.8 \mathrm{~K}$ compared to $2-4 \mathrm{~K}$ during solar maximum.
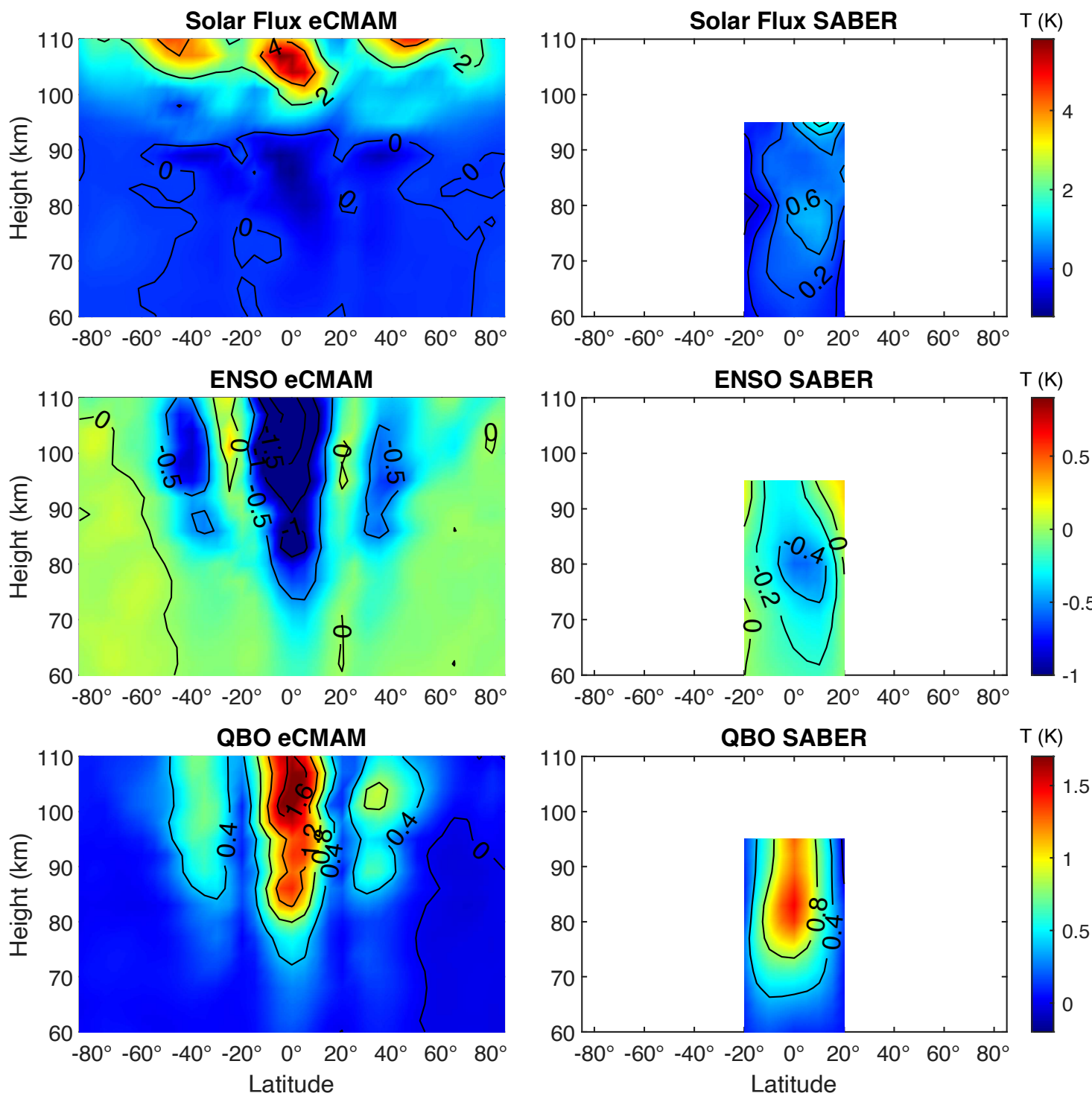

Figure 5.5. Coefficients of the DW1 T (K) amplitude as a function of height and latitude to the deterministic interannual variability indices, e.g., solar cycle (top panel), ENSO (middle panel), and QBO (bottom panel), in the eCMAM (left) and SABER (right). Note that the contour intervals are different for eCMAM and SABER and for different indices.

The middle panel in Figure 5.5 shows the response to ENSO in the eCMAM (left) and SABER (right). The coefficients show that the DW1 amplitude is generally negatively correlated with the ENSO indices in both eCMAM and SABER. When the ENSO is in warm-phase, e.g., El Niño, the DW1 amplitude is weaker; when the ENSO 
is in cold-phase - La Niña, the tidal amplitude tends to be stronger. DW1 amplitude is known to be stronger during La Niña years from previous studies (Gurubaran, 2005; Pedatella and Liu, 2012). At $\sim 80 \mathrm{~km}$, both datasets show the coefficients of 0.5 at the equator. The coefficients in eCMAM increase to 1.5 above $100 \mathrm{~km}$. Since the normalized ENSO indices change between -1 and 1, the actual ENSO response is about $-1.5 \mathrm{~K}$ for El Niño years and $+1.5 \mathrm{~K}$ for La Niña years.

Figure 5.5 bottom panel presents the coefficients of the tidal amplitude to the QBO indices in the eCMAM (left) and SABER (right). The response is positively correlated with the QBO indices, which means that when the QBO wind is westerly or eastward, the tidal amplitude is stronger, and on the other hand, when the QBO wind is easterly or westward, the tidal amplitude is weaker. Previous studies also presented that the DW1 amplitude tends to be greater during the westerly phase of the stratospheric QBO (Hagan et al., 1999b; Yamazaki et al., 2017). The magnitude of the coefficients in the eCMAM and SABER is comparable below $90 \mathrm{~km}$ and is between $\sim 0.4-1 \mathrm{~K}$. The coefficients increase to $1.6 \mathrm{~K}$ in eCMAM between 100 and $110 \mathrm{~km}$. The product of the coefficients and the normalized QBO indices (from -1 to 1) gives the actual tidal amplitude response to $\mathrm{QBO}$ at a particular time.

\subsection{Seasonal Variations of DW1}

This section presents the amplitudes for seasonal harmonics, e.g., the 12-, 6-, 4, and 3-months, in the regression model. Figure 5.6 shows the DW1 amplitudes (K) at the periods of 12, 6, 4, and 3 months in the eCMAM (left) and SABER (right). For the 12-month period (first row), both the model and observation show that the DW1 amplitudes maximize in the subtropics. The maximum amplitude is $\sim 4 \mathrm{~K}$ in eCMAM and $\sim 3 \mathrm{~K}$ in SABER. The subtropical maximum in SABER is stronger than that in the eCMAM between $70-90 \mathrm{~km}$. The DW1 amplitude at the 6 - month period (second row) presents the classic latitudinal structure of DW1 in eCMAM: maximum 



Figure 5.6. Amplitude of the DW1 $\mathrm{T}(\mathrm{K})$ variability as a function of height and latitude due to the seasonal harmonics: 12, 6, 4 and 3 months in the eCMAM (left) and SABER (right). 
over the equator with two secondary maxima at $35^{\circ} \mathrm{N} / \mathrm{S}$ between $90-100 \mathrm{~km}$. The maximum amplitude is $\sim 10 \mathrm{~K}$ and $2-3 \mathrm{~K}$ for the equatorial and mid-latitude maxima, respectively. SABER shows a similar equatorial maximum but with weaker amplitude $(5 \mathrm{~K})$ and at a lower height $(80-90 \mathrm{~km})$. The latitudinal structure and tidal amplitude of the 4-month harmonic in the eCMAM and SABER (third row) compare well below $90 \mathrm{~km}$ with maximum magnitude of $\sim 1 \mathrm{~K}$. Above $70 \mathrm{~km}$ in eCMAM, the latitudealtitude structure is similar to the 6 -month harmonic with an equatorial maximum and mid-latitude secondary maxima in each hemisphere. The equatorial maximum is skewed to $10^{\circ} \mathrm{S}$ with magnitudes of $\sim 3 \mathrm{~K}$ and the secondary maxima has magnitudes $\sim 1 \mathrm{~K}$. The comparison between the model and observation between $60-80 \mathrm{~km}$ is good for the 3-month harmonic: both eCMAM and SABER show magnitude of $0.6 \mathrm{~K}$ (fourth row). The maximum amplitude is present in the eCMAM equatorial region above $90 \mathrm{~km}$ with magnitudes of $\sim 1 \mathrm{~K}$.

\subsection{Long-term Variations in Short-term Tidal Variability}

As discussed in Chapter 1, seasonal variations of atmospheric tides can be impacted by propagation and forcing variations at other time scales such as ENSO, QBO, MJO, and solar cycle. However, the current understanding of the seasonal and interannual timescale impact on the short-term tidal variability is very limited. Since the characteristics in short-term DW1 tidal variability were presented in Chapter 4, the focus of this section is to shed new light into the long-term variations in short-term DW1 tidal variability. Short-term tidal variability is derived from the residue of the regression model by using a Fourier bandpass filter.

To identify the long-term variation in the short-term tidal variability, this topic is studied from two approaches. The first approach is to use absolute variability, e.g., the standard deviation, in the short-term tidal variability on a monthly basis to examine if there is any long-term variation. The second approach is to use relative 
variability, which is the absolute variability normalized by the monthly mean from a particular data window. The results from both approaches are shown in Figure 5.7 for the eCMAM. Figure 5.7 first panel shows the absolute variability of the shortterm tide as a function of month from January 1979 to December 2009 at $100 \mathrm{~km}$ and the equator. The Fourier spectrum of the absolute variability is present in the second panel. The significance levels are estimated from the Monte Carlo simulation by using 1000 random time series with the same mean and standard deviation as the short-term tidal data. The Fourier analysis shows peak values at seasonal (6- months and 12- months) and QBO (27-28 months) time scales. Significance test shows that the 6-months signal is well above the $95 \%$ level (orange line) against white noise. The 12-months signal is also above the $95 \%$ level. The other peaks are below the significance test which may attribute to the relative short data length (372 months) used here.

The relative variability is presented in the third panel of Figure 5.7. There are significant month-to-month variations in the relative variability of the short-term tidal amplitude. It varies between a few percent to $25 \%$ for the majority of the months, while December 1999 peaks at 45\%. The fourth panel in Figure 5.7 presents the Fourier spectrum of relative variability. The Fourier spectrum also shows peaks at 6month, 12- month, and QBO time scale, although the 12-month and QBO seems to be relatively weaker compared to the 6-month peak than those shown in the absolute variability. The 6 -month peak is the only peak that is high above the $95 \%$ significance level (orange line). We performed a similar analysis on the SABER short-term tide from January 2003 to December 2016 at $85 \mathrm{~km}$ and the equator. Both absolute and relative variability shows significant peaks at the 6-month period (figures not shown). 

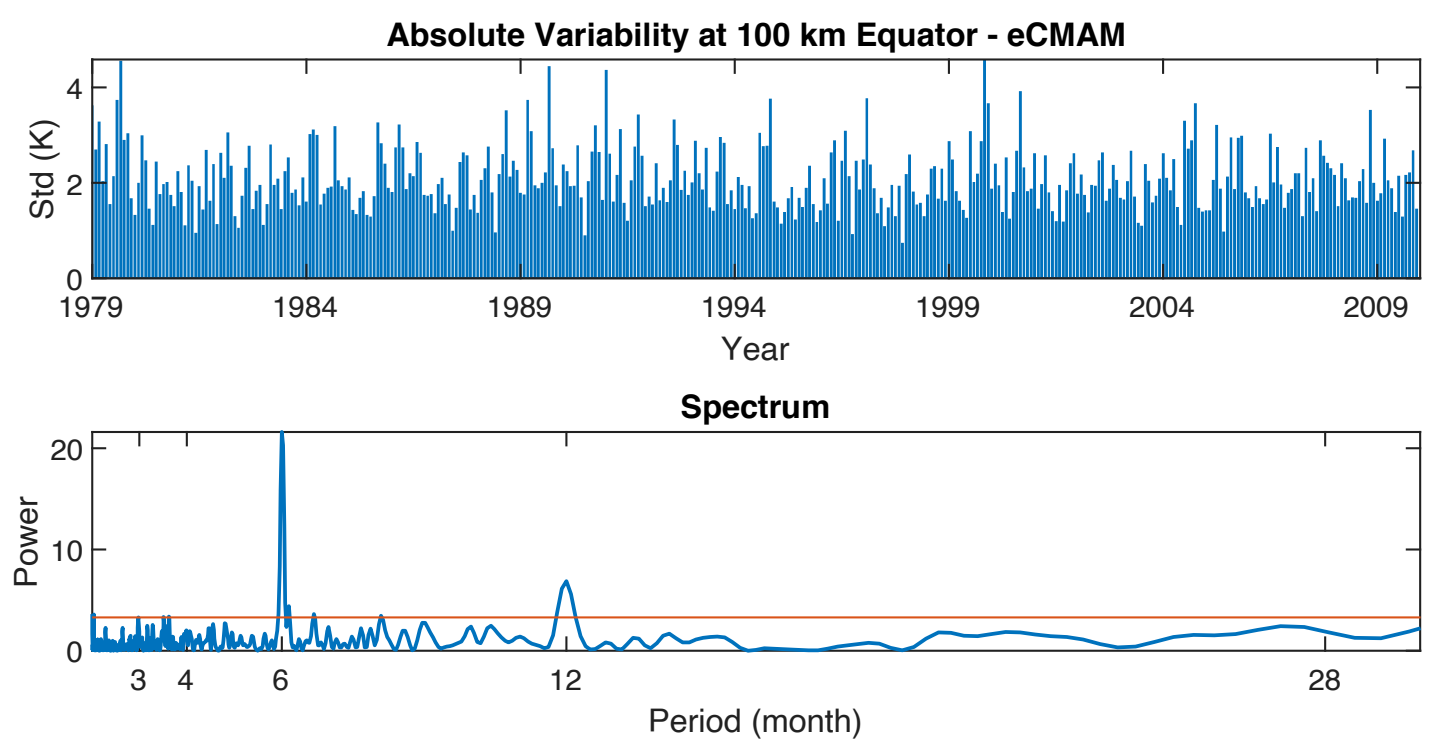

Relative Variability

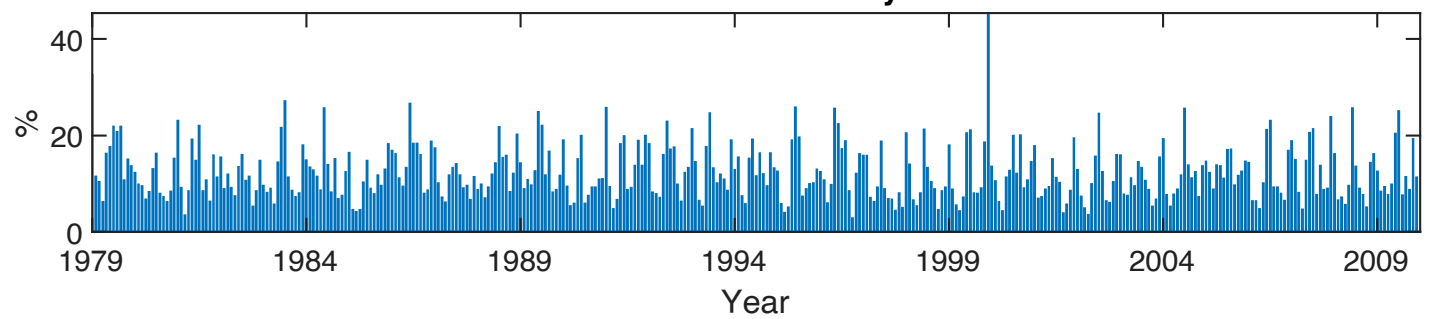

Spectrum

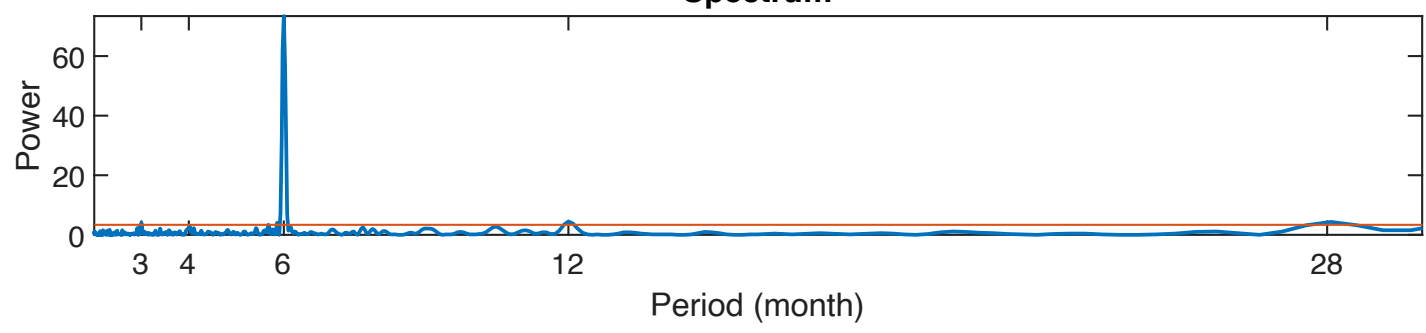

Figure 5.7. Monthly absolute variability of the short-term DW1 tidal amplitude in eCMAM at $100 \mathrm{~km}$ and the equator (first panel) and its Fourier spectrum (second panel). Similar as the first two panels, but for monthly relative variability (third panel) and the corresponding Fourier spectrum (fourth panel). The orange line is the 95\% significance test with Monte Carlo of 1000 white noise time series.

Next, focus on the 6-month variation in the short-term tidal amplitude and analyze to what extent it is related to the seasonal variation of the tide. As discussed in Section 5.5, DW1 presents strong 6-month (semi-annual) variation with stronger tidal amplitude in March and September and weaker amplitude in June and December. All of the absolute variability from March (30 in eCMAM, and 14 in SABER) are 
grouped into a box plot to examine the distribution of data based on a five number summary (minimum, first quartile (Q1), median, third quartile (Q3), and maximum). Same method is applied for June and then repeated for the relative variability. Figure 5.8 top left panel illustrates the absolute variability from the eCMAM forMarch and June. The absolute variability varies between $0.5-1.4(0.33-0.9) \mathrm{K}$ with a median of $\sim 0.9$ (0.6) $\mathrm{K}$ for March (June). The first and third quartiles are vary between 0.79 to 1.1 (0.5 to 0.76$) \mathrm{K}$ in March (June). It is clearly shown that the absolute variability in March presents larger values than that in June. Student's t-test is performed to examine if the two groups of absolute variability from March and June are significantly different from each other based on the mean and standard deviation. It has passed the Student's t-test. This implies that the absolute variability is larger when mean tidal amplitude is stronger, and vice versa.

For SABER (Figure 5.8 bottom left panel), the absolute variability ranges from 0.6 to $1.55 \mathrm{~K}$ for March and from 0.2 to $1.4 \mathrm{~K}$ in June. The median absolute variability is $\sim 1 \mathrm{~K}$ in March and $\sim 0.8 \mathrm{~K}$ in June. The range for the first quartile and third quartile for March is slighter higher and for June as well. However, the absolute variability for March and June from SABER did not pass the Student's t test, which possibly due to the sample size (14 for SABER).

The box plot for the relative variability shows the opposite picture from the absolute variability from both the eCMAM (Figure 5.8 top right panel) and SABER (Figure 5.8 bottom right panel). Both eCMAM and SABER show that the relative variability shifts to larger values in June than that in March. DW1 short-term tide in the eCMAM has relative variability up to $~ 9 \%$ in March comparing to maximum variability of $35 \%$ in June. The median relative variability is $\sim 6 \%$ in March compared to a median of $19 \%$ in June. The relative variability in SABER is up to $\sim 8 \%$ in March comparing to $26 \%$ in June. The median relative variability is $\sim 6 \%(\sim 12 \%)$ in March (June). The two groups of relative variability for March and June from both 
the eCMAM and SABER passed the Student's t test. This implies that short-term tidal variability in June is more variable relative to its mean tidal amplitude than that in March, despite the mean tidal amplitude being stronger in March than June.
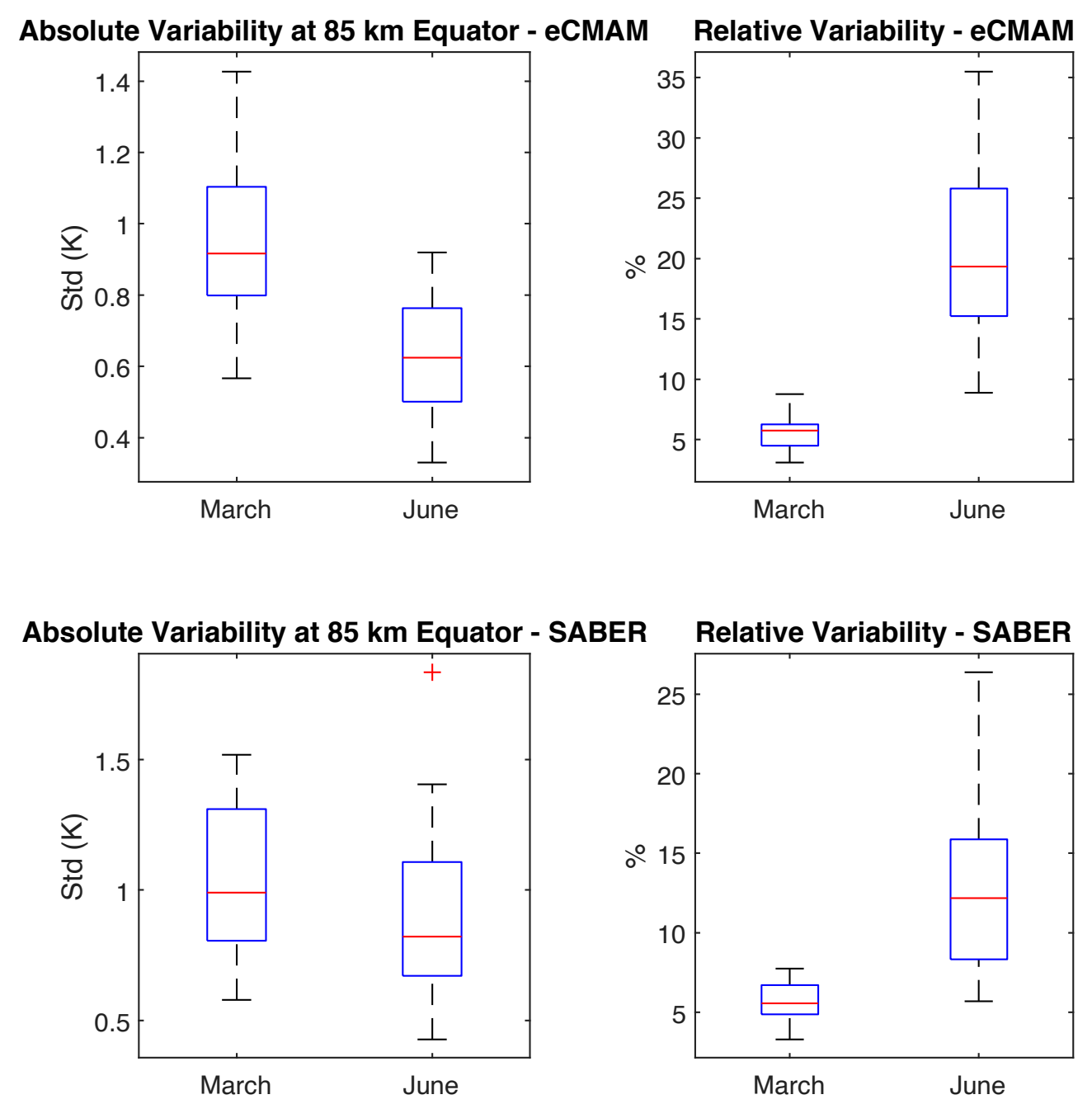

Figure 5.8. Box plot of the absolute variability in March and June for eCMAM (top left), SABER (bottom left) and relative variability for eCMAM (top right) and SABER (bottom right) at $85 \mathrm{~km}$ equator. 


\subsection{Relative Importance of Temporal Variability}

As shown in previous sections, temporal variability in atmospheric tides varies on a range of time scales from short-term (periods $<30$ days) to long-term (several months to years). In this section, we will investigate the relative importance that different scales of temporal variabilities play in the total tidal variability.

Figure 5.9 illustrates the DW1 tidal variability in different temporal scales in 2009 from the eCMAM data. The first panel shows the DW1 total tidal variability at $95 \mathrm{~km}$. The second, third, and fourth panel shows the tidal variability in interannual (periods $>1$ year), seasonal and intraseasonal (30 days $<$ periods $<1$ year), and shortterm (periods $<30$ days) time scale, respectively. The total DW1 amplitude varies in the range of $-12-13 \mathrm{~K}$ over one year (first panel). Inter-annual variability anomaly varies in the range of $-2.8-1.2 \mathrm{~K}$ (second panel) and this range is $\sim 15 \%$ of the total variability range. Seasonal and intraseasonal tidal anomaly (third panel) varies between $-9-9 \mathrm{~K}$ and is $\sim 72 \%$ of the total variability. Short-term tidal variability (fourth panel) varies between $-8-7 \mathrm{~K}$, which is $\sim 59 \%$ of the total variability range.

Apparently, the relative importance of variability from different time scales depends on the data length under investigation. For example, the effect of inter-annual variability would be negligible when we study tidal variability in a few days. On the contrary, short-term tidal variability would play a minor role when we study tidal variability on a climatological scale. Hence, it is important to assess the characteristic of the tidal variability from different time scales as a function of data length. Relative Variance (RV) is calculated as a ratio of the variability variance from a specific time scale range divided by the variance from all time scales over the same time window (Hermance, 2014). For example, the RV for the short-term tidal variability (period 

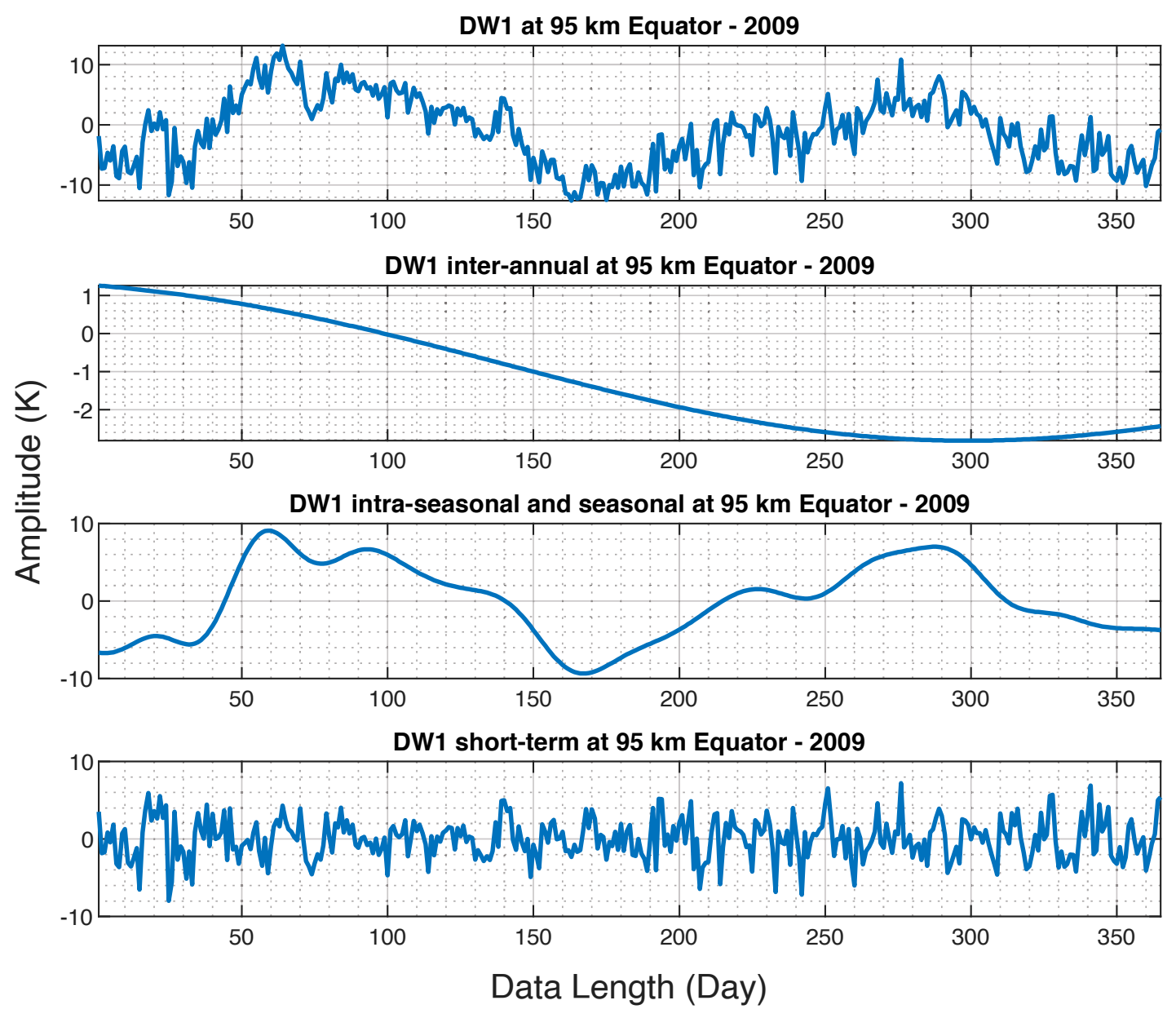

Figure 5.9. DW1 total tidal variability (first panel), tidal variability in interannual (second panel), seasonal and intraseasonal (third panel), and short-term (fourth panel) time scales at $95 \mathrm{~km}$ in 2009.

$<30$ days) is calculated as:

$$
R V_{\text {short }}=\left(\frac{\text { variance }_{\text {short }}}{\text { variance }_{\text {total }}}\right) \times 100
$$

where $_{\text {variance }_{\text {total }}}=$ variance $_{\text {short }}+$ variance $_{\text {seasonal }}+$ variance $_{\text {interannual }}$. RVs for $_{\text {f }}$ seasonal and interannual variability are calculated by replacing the variance of shortterm with seasonal and interannual variability on the numerator, respectively, while the denominator remains unchanged.

We started to calculate RVs from short-term, seasonal, and inter-annual variability for a data length of 7 days. The same data window is then stepped forward by one 
day a time to cover the whole available data from SABER (January 2003 - December 2016) and eCMAM (January 1979 to June 2010). The average RV is then calculated for that data window (7 days here) and presented in Figure 5.10. We repeated these processes for data lengths of 14,21, 30, 60, 90, 120, 180 and 365 days. Figure 5.10 shows the average RVs of short, seasonal, and interannual variability at $95 \mathrm{~km}$ equator in both eCMAM (solid lines) and SABER (dashed lines) data as a function of different data lengths.

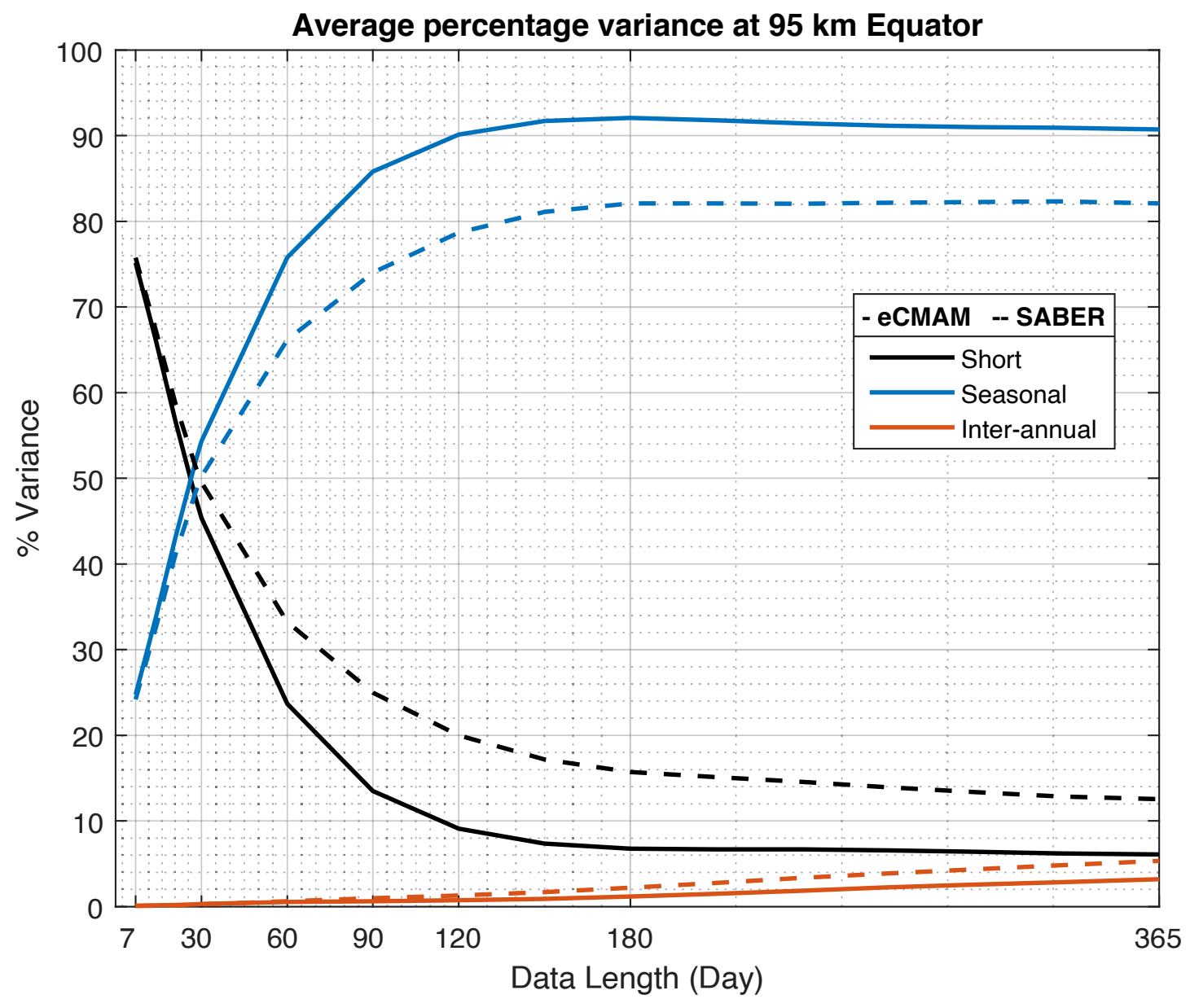

Figure 5.10. Average relative variance at $95 \mathrm{~km}$.

For data length of 7 days, short-term tidal variability contributes $\sim 75 \%$ of the total tidal variability in both eCMAM (solid black line) and SABER (dashed black line). The contribution of short-term variability gradually decreases to $50 \%$ for the 
time window of 30 days in SABER compared to $45 \%$ in eCMAM. It decreases to $33 \%$ at the data length of 60 days in SABER and 23\% in eCMAM. The decreasing rate becomes smaller for data length longer than 90 days. The RV contribution from the short-term tidal variability decreases to $\sim 13 \%$ in SABER and $\sim 6 \%$ in eCMAM for data length of 365 days. The relative contribution of the short-term tidal variability to the total tidal variability is similar for the time window $<30$ days in SABER and eCMAM, while SABER is $6-12 \%$ higher than the eCMAM for the time window $>60$ days.

Seasonal variability contributes to $\sim 25 \%$ of the total tidal variability in both eCMAM (solid blue line) and SABER (dashed blue line) for the data length of 7 days. Seasonal variability starts to dominate in the total tidal variability after 30 days and persists with the increase of data length in both datasets. For the data length of 60 days, the RV of seasonal variability increases to $~ 75 \%$ in eCMAM and $\sim 65 \%$ in SABER. After 120 days, both eCMAM and SABER show nearly constant RV at $\sim 92 \%$ and $82 \%$, respectively.

$\mathrm{RV}$ of the interannual variability in both eCMAM and SABER increases with the increasing data length. However, it remains negligible $(<1 \%)$ for the time window $<180$ days for both datasets. Interannual tidal variability contributes $2 \%$ of the total tidal variability for the time length of 180 days in SABER (dashed orange line) and $1 \%$ for eCMAM (solid orange line). The contribution of the interannual variability increased to $\sim 5 \%$ in SABER and $\sim 3 \%$ in eCMAM for the time window of 365 days. Interannual variability increases to $10 \%$ in SABER and $7 \%$ in eCMAM for the time window of 14 years (not shown in Figure 5.10).

\subsection{Total Tidal Forecast}

As noted in Chapter 1, in geomagnetic quiet conditions, variations in the IT system are significantly perturbed by the energy input from upward propagation of waves 
(including tides, planetary waves and gravity waves) generated in the troposphere and stratosphere. It is important to forecast the lower atmospheric tidal variability accurately in order to improve the space weather predictions in the TI system. Recent research indicates that intra-seasonal, seasonal, and interannual variability in the lower atmosphere (such as MJO, QBO, and ENSO) offer potential new sources of whole atmosphere predictability (Sassi et al., 2019). The goal of this section is to develop a model to predict the total tidal variability of DW1 based on knowledge from Chapter 4 and from earlier this Chapter.

$$
\text { Total Tidal Forecast }=\begin{gathered}
\text { Multiple Linear Regression } \\
\text { Model }
\end{gathered}+\begin{gathered}
\text { Tidal Weather } \\
\text { Forecast }
\end{gathered}
$$

Chapter 4 presents a model to forecast the tidal weather of DW1. The seasonal and interannual parameters obtained from the multiple linear regression model in sections 5.4 and 5.5 can be used to fit the long-term tidal variability. Although the total tidal variability forecast model is a linear combination of the long term fitting terms and the short-term tidal variability forecast model. However, the shortterm tidal variability forecast model has long-term information built in it. The 23 coefficients used in this model are calculated from 7 years of data. 23 day data prior to the forecast needed also carries long time information, for example, are the 23 day of data from March or September? Are these data from QBO westerly phase years or easterly?

Figure 5.11 left panels show the actual long-term, short-term and total variability of DW1 temperature amplitude (in K) at the equator in 2009 as a function of height and day of the year. The right panels show the corresponding long-term variation fitted from the multiple linear regression model based on Equation 5.1, short-term variability prediction from Chapter 4 at day-1, and the predicted total tidal variability according to Equation 5.3, e.g., by adding the right top two panels together. 

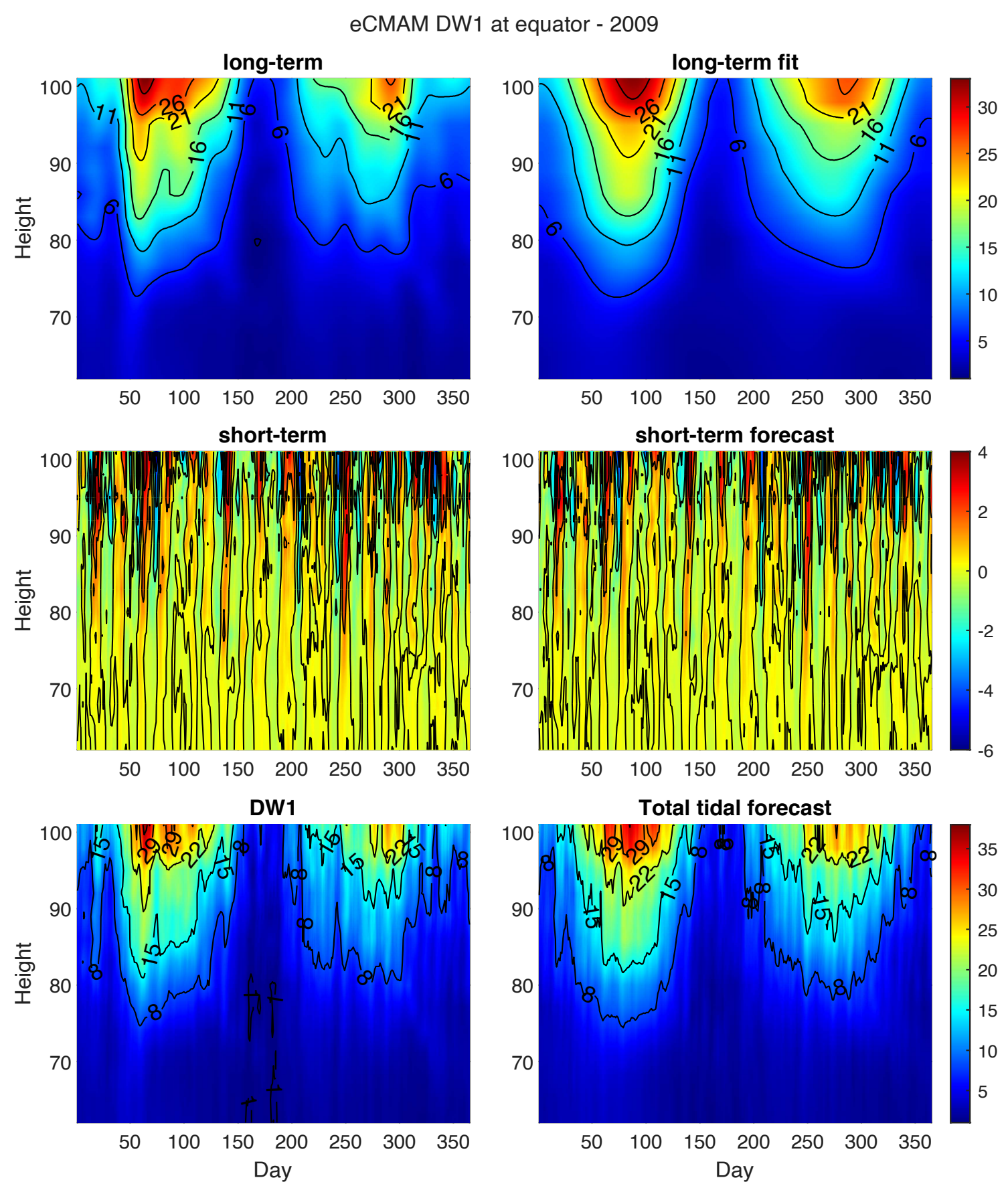

Figure 5.11. Actual long-term (top left), short-term (middle left), and total variability (bottom left) and fitted long-term (top right), predicted short-term (middle right), and predicted total variability (bottom right) of eCMAM DW1 T amplitude (in K) at the equator in 2009 as a function of height and day of the year.

Both the actual long-term (left panel) and long-term fit (right panel) show the semiannual variation of the tides with higher temperature around March and Septem- 
ber. However, long-term fit is smoother compared to the actual long-term tidal variability, which implies that there are other long-term variabilities not being captured by the regression model. The actual short-term tidal variability and the forecasted short-term tidal variability show similar features. The total forecasted tidal variability shows a clearer defined semiannual variability than the actual tide.

Figure 5.12 is similar to Figure 5.11, but for SABER DW1 at the equator in 2014. Both the actual long-term and long-term fit shows semiannual variability. The double peak feature in March around $80-85 \mathrm{~km}$ is also not captured by the model. Similar characteristics are also observed with the total tidal variability (Figure 5.12 bottom panel).

To quantify how accurate the forecasted total tidal variability based on Equation 5.3 compared to the actual tidal variability, we calculated R-squared values (correlation coefficients squared) between the forecasted and actual time series for each year at day-1 prediction. Figure 5.13 presents the prediction accuracy $\left(R^{2}\right.$ values) of the total tidal forecast as a function of year at $95 \mathrm{~km}$ for the eCMAM (blue line) and SABER (red line) data. As described in Chapter 4, 2000 data points (about 7 years) are needed in the AR model to get a robust prediction in the short-term tidal variability. Hence Figure 5.13 shows eCMAM forecast accuracy from 1985 to 2009 and SABER from 2009 to 2016. The first panel shows the modeled accuracy for the long-term variability of the tide. The highest $R^{2}$ value is $\sim 0.97$ in 1990 (which means correlation coefficient is $\sim 0.98$ ) and the lowest value is $\sim 0.64$ in 2005 (correlation coefficient is $\sim 0.8$ ). The overall prediction accuracy is generally lower in SABER than eCMAM. The highest $R^{2}$ value for SABER is $~ 84 \%$ in 2011 (correlation coefficient is $\sim 0.91$ ) and the lowest prediction accuracy at around 50\% in 2012 (correlation coefficient is $\sim 0.7$ ). The second panel shows the $R^{2}$ values for the short-term tidal forecast. The short-term tidal prediction at day-1 for SABER is as high as eCMAM, both $R^{2}$ is around 0.9. Prediction accuracy for the total tide follows the pattern of 

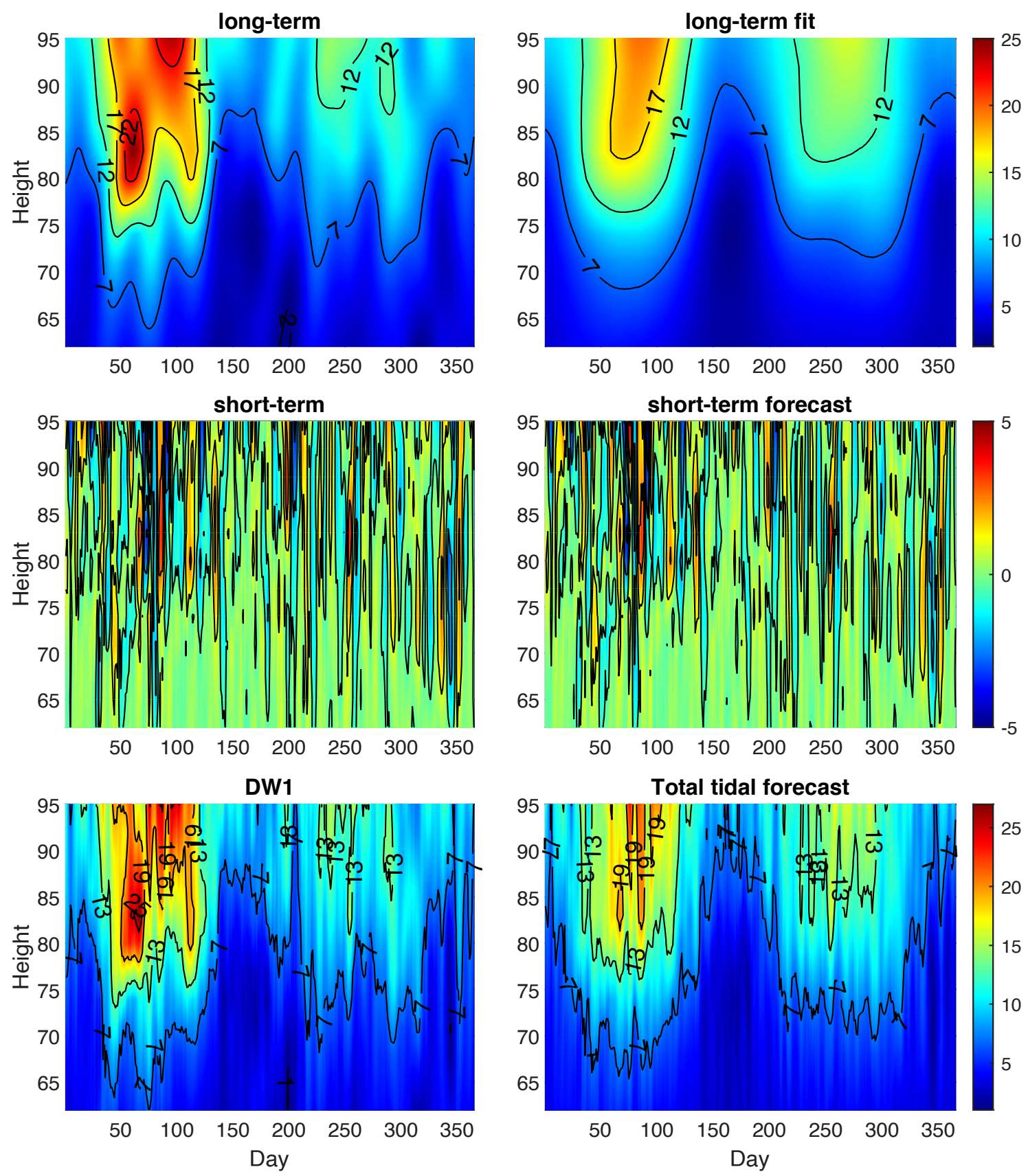

Figure 5.12. Actual long-term (top left), short-term (middle left), and total variability (bottom left) and fitted long-term (top right), predicted short-term (middle right), and predicted total variability (bottom right) of SABER DW1 T amplitude (in K) at the equator in 2014 as a function of height and day of the year. 

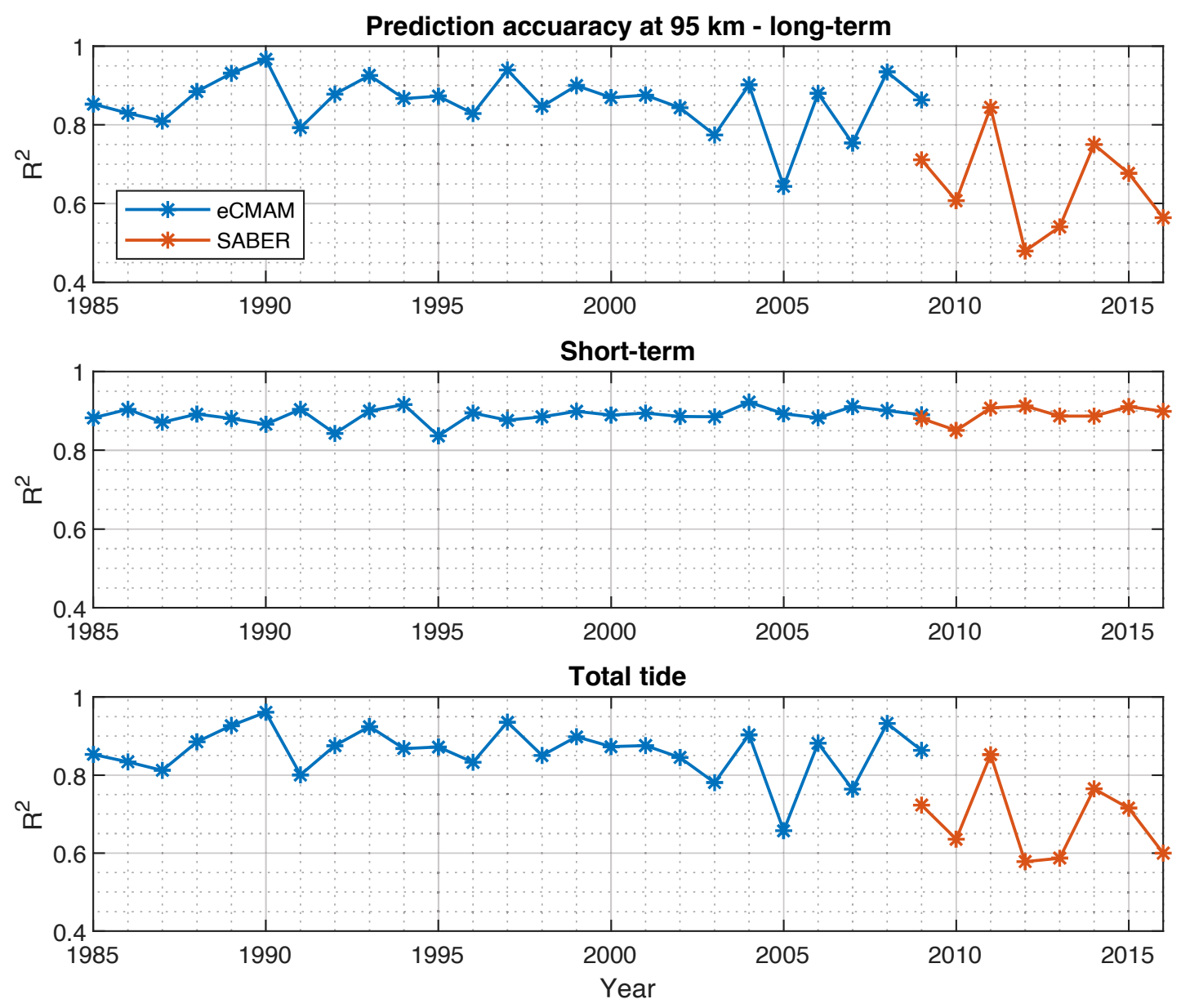

Figure 5.13. Predicted accuracy of the long-term (top), short-term (middle), and total tide (bottom) at $95 \mathrm{~km}$ equator for eCMAM (blue line) and SABER (red line).

the long-term variability. The highest $R^{2}$ for the total tide is $\sim 0.97$ in 1990 and the lowest is $\sim 0.65$ in 2005 in the eCMAM. SABER has the highest $R^{2}(\sim 0.85)$ in 2011 and lowest (0.57) in 2012. The reason for lower prediction accuracy in SABER mainly lies in the long-term fit rather than the short-term tidal prediction.

Next, we examine the best and worst $R^{2}$ values from both datasets in more detail to investigate the possible discrepancy of the model. Figure 5.14 shows the tidal variability at $95 \mathrm{~km}$ (left) and its periodogram (right) for the highest and lowest predicted years in eCMAM (upper two panels) and SABER (lower two panels). The first row presents the eCMAM DW1 total tidal variability and the predicted tide at $95 \mathrm{~km}$ in 1990 (best $R^{2}$ year). The Fourier spectrum shows that the regression model captured 
all of the tidal variability very well with an accuracy of $\sim 97 \%$. The second row shows the actual and predicted tide and its Fourier spectrum in 2005. The prediction accuracy in 2005 is the lowest in eCMAM $\left(R^{2}=0.66\right)$. The spectrum shows that the eCMAM DW1 in 2005 has a relatively strong peak between 3 to 4 months which is not captured by the regression model since the model only includes periods at the exact 3 and 4 months.

Third row of Figure 5.14 shows the actual DW1 amplitude, the predicted DW1 and the spectrum for SABER in 2011 (best $R^{2}$ value: 0.84). The spectrum for the actual and predicted tides matches well for periods shorter than 7 months, however, there are relatively strong peaks between $7-12$ months that the regression model 

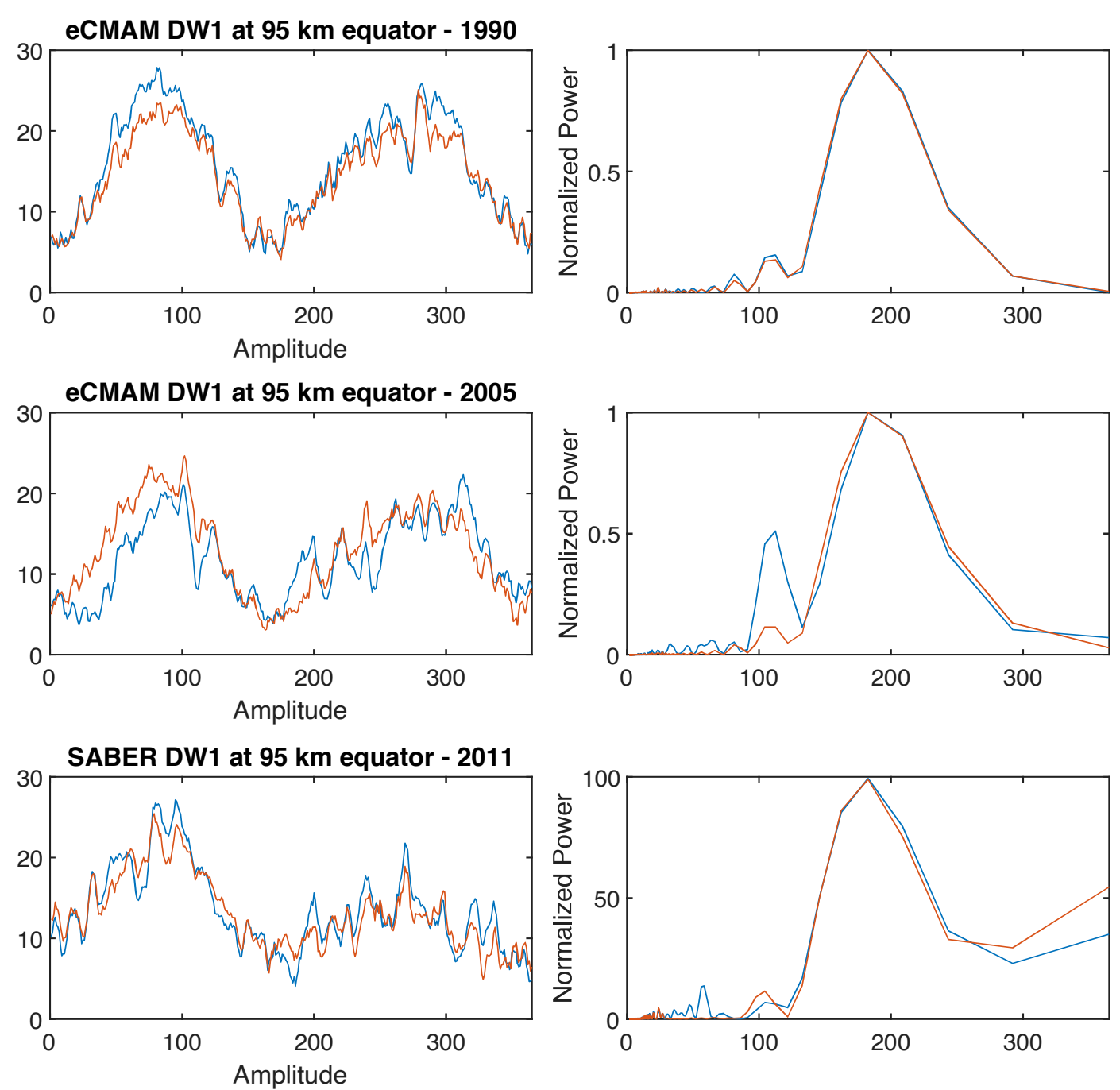

SABER DW1 at $95 \mathrm{~km}$ equator - 2012
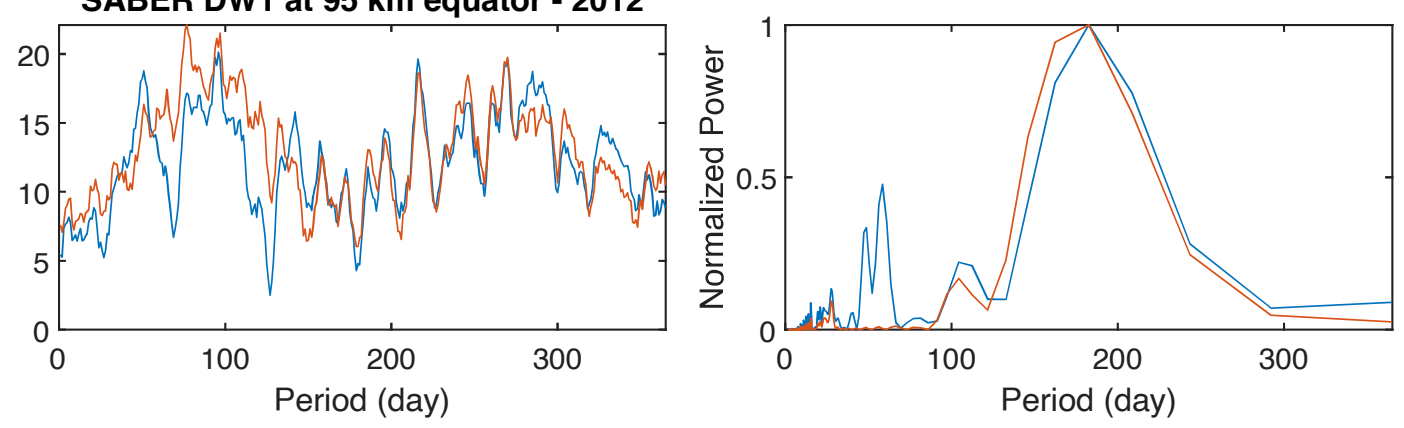

Figure 5.14. eCMAM DW1 tide in 1990 (first row), 2005 (second row), SABER 2011 (third row), and 2012 (fourth row) with respective Fourier spectrums.

does not capture. The fourth row shows the SABER DW1 in 2012 (worst $R^{2}$ value: 0.50). The Fourier spectrum reveals that there is a relatively high peak around $40-60$ 
days which are not captured by the model. This investigation shows that besides the well-known periods at which the tide oscillates, there are variations at other periods as well and they can also change significantly on a year-to-year basis.

\subsection{Discussion and Conclusions}

We focus our study on the total tidal variability In this chapter. Wavelet analyses of the DW1 reveal that temporal variability of the tide has a rich spectrum in both the eCMAM and SABER. Tides are known to oscillate at interannual, seasonal, interseasonal, and short-term periods. Hence, we first fit the data with a multilinear regression model that contains deterministic variability such as the solar cycle, ENSO, QBO, and the seasonal harmonics at 12-, 6-, 4-, and 3- months. The fitting coefficients/amplitudes from each deterministic variability are examined. Both the model and SABER shows that the DW1 amplitude is stronger in the solar maximum years than the minimum years. The DW1 amplitude is stronger during the coldphase ENSO (La Niña) years than the warm-phase (El Niño) years. The results are in agreement with previous studies (Gurubaran, 2005; Pedatella and Liu, 2012). The ENSO-driven variability in the DW1 is found to be primarily due to changes in the tropospheric forcing of the DW1 (Pedatella and Liu, 2013b). The DW1 T amplitude is stronger in the westerly phase of the QBO with zero lag than in the easterly phase. Similar QBO modulations of DW1 have been observed in the MLT region in both horizontal winds (Dhadly et al., 2018) and temperature (Xu et al., 2009). For the seasonal harmonics in the equatorial region, both the model and SABER show that the 6-month harmonic has the strongest amplitude, and is followed by the 12-month harmonic. The 4- and 3-month harmonics are the smallest. The amplitudes follow a similar order for the mid-latitude maxima in the eCMAM.

Index products such as QBO*ENSO, QBO*Solar, and ENSO*Solar are also included in the regression model to exam the effect of the mixed terms (Kumari and 
Oberheide, 2020). The sign of the original coefficients remains unchanged, and the magnitude of the coefficients slightly changed. The overall fit is relatively invariant evaluated from the correlation coefficients between the mixed terms and the total tidal variability (from 0.835 to 0.845 ). Different time lags (1-7 months) are added in the ENSO index in the regression model, the sign of the coefficients remain unchanged with the magnitude of the coefficients getting smaller with increasing time lag. The overall fit is still very similar to the original regression model with ENSO lag $=0$ month.

One of the outstanding questions about short-term tidal variability is whether long-term variations in the atmosphere such as solar variability, ENSO, QBO, and seasonal variation can also modulate the short-term tidal variability. We investigate this question by examining both the absolute and relative variability of the shortterm tidal variability on a monthly basis. Absolute variability is the actual standard derivation of the time series over one month window, and relative variability is the absolute variability weighted by the monthly mean amplitude of the tide. Both absolute and relative variability of the short-term tidal time series show significant long-term variations. The 6-month variation is the most prominent. We fitted a box plot to the absolute and relative variability from all March and June in the eCMAM and SABER. The results are rather surprising. The absolute variability shows that variability is larger in March when the mean tidal amplitude is stronger and smaller in June when the mean tidal amplitude is smaller. However, the relative variability shows an opposite picture. The relative variability is larger in June than March, which implies that short-term tidal amplitude varies more relative to its mean when the tidal mean amplitude is weaker. Student's t-test is also performed and the absolute variability and relative variability in eCMAM and relative variability in SABER passed the significant test and have significantly different distributions. However, the absolute variability in SABER did not pass the student's t-test. 
There has been very little research on long-term variations of short-term tidal amplitudes in the MLT region. Kumari and Oberheide (2020) discussed the statistical characteristics of interannual changes in short-term DE3 (symmetric and antisymmetric DE3 tidal modes) variability on a quasi-10-day wave (Q10DW) timescale and related it to various forcing and propagation conditions such as QBO, ENSO, and solar cycle. Symmetric DE3 mode variability is enhanced during the easterly phase of the QBO due to enhanced Q10DW activity and during the La Niña phase of ENSO due to enhanced convective forcing. Antisymmetric DE3 mode variability is enhanced in the westerly phase of the QBO during solar maximum due to Q10DW activity related to a more unstable polar vortex in the northern hemisphere. The community has certainly not reached a consensus on how and why such tidal variability happens. It is challenging to make the distinction whether these variabilities are caused directly by lower atmospheric processes (MJO, QBO, and ENSO) or an indirect result of wave-mean flow interaction during the tidal upward propagation, or nonlinearity of the atmospheric response through wave-wave interaction. The long-term temporal structures in the short-term tidal variability need an extensive investigation in the future.

Since tides exhibit variability from daily to solar cycle time scales in the MLT region, it is crucial to assess the relative contribution of tidal variability at different time scales. Obviously, their relative contributions are a function of time length. We investigated these contributions at time lengths of $7,14,21,30,60,90,120,180$ and 365 days. Short-term tidal variability contributes $\sim 50-75 \%$ of the total tidal variability between 7 to 30 days in SABER and eCMAM. SABER shows a higher amount of short-term variability compared to the eCMAM. Both the model and SABER agree that the seasonal variability (between 3 and 12 months) accounts for the largest variability (70-90\%) for time length greater than 60-days. The relative contribution from interannual tidal variability remains small and increases to less 
than $5 \%$ at time length of 365 days.

We end this chapter by predicting the total time series of DW1 T amplitude using the coefficients obtained in the regression model (long-term variability) and the tidal weather forecast from the AR model (Chapter 4). For the total tidal forecast at the $95 \mathrm{~km}$ and the equator, eCMAM showed the highest $R^{2} \sim 0.97$ in 1990 and the lowest $R^{2} \sim 0.65$ in 2005 . The highest $R^{2}$ for the total tidal forecast in SABER is $\sim 0.85 \mathrm{in}$ 2011 and the lowest is $\sim 0.57$ in 2012 . The relatively low $R^{2}$ for some years is mainly due to the regression model not capturing all the long-term tidal variability ( $>30$ days), since the short-term tidal forecast in both eCMAM and SABER have very high $R^{2}$ values $(\sim 0.9)$. 


\section{CHAPTER 6}

\section{PHYSICAL MECHANISM STUDY OF THE SHORT-TERM TIDAL VARIABILITY IN THE MESOSPHERE AND THERMOSPHERE}

The objective of this chapter is to examine how linear advection (wave-mean flow interaction), nonlinear advection (wave-wave interaction), and solar heating (shortwave heating) govern the short-term variability of DW1 in the mesosphere and thermosphere. The chapter is organized as follows. Section 6.1 presents the current understanding of the DW1 tidal mechanisms in the mesosphere. In section 6.2, The possible mechanisms that govern the short-term tidal variability are investigated including linear advection, nonlinear advection and solar heating. Then investigated the role of the five terms in the linear advection in the mesosphere is investigated. Non-local effects from solar heating are also studied. Section 6.3 presents the physical mechanisms of the short-term tidal variability of DW1 in the thermosphere. This chapter closes with discussion and conclusions in Section 6.4. The latitudinal coverage is limited to between $45^{\circ} \mathrm{S}-45^{\circ} \mathrm{N}$ since DW1 is prominent in this region.

\subsection{Introduction}

As presented in previous chapters, DW1 is known to display different spatial structures and temporal variability over inter-annual, seasonal, intra-seasonal and shortterm time scales and can thus contribute to the variability of the space environment. 
The DW1 tidal amplitude undergoes a substantial semiannual variation in the mesosphere, which is characterized by stronger amplitudes at the equinoxes and weaker amplitudes at the solstices. Propagation modulation due to altered background mean wind, variation in the heating sources that force this tidal component, and the nonlinear interaction with planetary waves, gravity waves or both are the physical mechanisms that have recently proposed to explain the observed semiannual variation of DW1 (Lieberman et al., 2010; McLandress, 2002a,b). Though several modeling studies have addressed the observed seasonal variations in DW1, underlying physical causes are still under debate (Akmaev, 2001; Gu and Du, 2018; Hagan et al., 1995, 1999a; McLandress, 2002a, 1997, 2002b).

A detailed analysis of the terms in the tidal momentum and thermodynamic budgets have been used to establish direct cause and effect of DW1 in the mesosphere (Lieberman et al., 2010; Lu et al., 2012; McLandress, 2002b; Gu and Du, 2018). McLandress (2002b) analyzed the budgets of DW1 using climatological simulation from the eCMAM at two latitudes $\left(20^{\circ} \mathrm{N}\right.$ and $\left.20^{\circ} \mathrm{S}\right)$ and found that the linear advection terms which involve the products of zonal mean quantities and perturbations play an essential role in the seasonal variation of the tide. Lu et al. (2012) followed the same methodology in their study using the Whole Atmosphere Community Climate Model version 4 (WACCM4) and concluded that both gravity wave (GW) forcing and the linear advection are important in determining the zonal wind structure of DW1 in March. The terms of the momentum budget of the DW1 were diagnosed in Lieberman et al. (2010) using the temperatures and geopotential height from SABER and the horizontal wind vector provided by the TIDI instrument. Between 85 and $100 \mathrm{~km}$, leading nonclassical terms in the momentum budget of DW1 are the meridional advection of zonally averaged momentum and wave drag effects encapsulated in the momentum residuals. Equation 6.1 is the zonal momentum equation (see Section 2.4). Sakazaki et al. (2013) investigated the seasonal variations of DW1 from 
the stratosphere to the lower mesosphere using Modern Era Retrospective analysis for Research and Applications (MERRA) and a linear tidal model. For the seasonal variation, the amplitude in the stratosphere maximizes at solstices. However in the lower mesosphere at $64 \mathrm{~km}$, the amplitude tends to maximize at the equinoxes. In addition to the DW1 excited by diabatic heating, secondary excitation by meridional advection of background zonal momentum have a significant contribution to the DW1, creating the above-mentioned seasonal variation from the stratosphere to the lower mesosphere in the tropics.

$$
\begin{aligned}
\frac{\partial u}{\partial t}= & f v-\frac{1}{a \cos \phi} \frac{\partial \Phi}{\partial \lambda}-\vec{v} \cdot \nabla u+u v \frac{\tan \phi}{a} \\
& +F_{G W D, x}+F_{D i f f, x}+F_{\text {ion }, x}+F_{\text {other }, x}
\end{aligned}
$$

$\mathrm{Gu}$ and $\mathrm{Du}$ (2018) investigated the seasonal variability of the thermodynamic budget of DW1 in the stratosphere, mesosphere, and lower thermosphere up to $200 \mathrm{~km}$ using 31.5 year $(1979-2010)$ output from eCMAM with latitude range between $85^{\circ} \mathrm{S}$ and $85^{\circ} \mathrm{N}$. DW1 annual variation in the stratosphere is mainly governed by the shortwave heating in the high latitudes, but by both the short-wave and adiabatic heating in the low latitudes. In the mesosphere, linear and nonlinear advection play important roles on the semiannual variation of the tide, whereas short-wave heating does not. In the lower thermosphere, the annual variation of DW1 is mainly governed by the short-wave heating and linear advection. Their study illustrates the complexity of the main physical mechanisms modulating the seasonal variations of DW1 in different regions of the atmosphere.

Moreover, the DW1 tide also exhibits strong interannual variation closely related to the stratospheric quasi-biennial oscillation (QBO). Mayr and Mengel (2005) suggested that the diurnal tide QBO effect is related to the mesospheric mean zonal wind QBO-like variations caused by the direct link via the advection terms in the tidal equation. The mechanisms responsible for generating interannual variability in 
the zonal mean and migrating and nonmigrating tides in the MLT due to ENSO were investigated using the WACCM and GSWM models by Pedatella and Liu (2013b). In the MLT, the DW1 enhancement is most notable during El Niño time periods and is driven by anomalously large tropospheric radiative forcing of the DW1. Yang et al. (2018) investigate the physical processes involved in the response of the mesosphere to observed MJO events using a simulation from the Specified-Dynamic Whole Atmosphere Community Climate Model (SD-WACCM) and show that a significant connection exists between the MJO and the mesospheric DW1 tidal amplitude. The DW1 tidal amplitudes of $\mathrm{T}$ in the equatorial mesosphere and the amplitudes of $\mathrm{U}$ and $\mathrm{V}$ in the southern subtropical mesosphere are significantly enhanced during MJO phase 1 to phase 3 periods. The MJO can affect the DW1 forcing by modulating both the solar insolation absorption and latent heat release in the equatorial troposphere and the background wind (propagation conditions). The perturbations of DW1 tidal amplitude in the troposphere propagate into the mesosphere and have a direct effect on the DW1 tidal amplitude there.

The plausible causes for the short-term tidal variability are variations in the background wind, variability in the tidal sources, and nonlinear interactions. Chang et al. (2011) studied the short-term variability in the migrating diurnal tide caused by interactions with the quasi two-day wave (QTDW) using the TIME-GCM. The QTDW induced changes in the tidal linear advective tendency are larger than changes in the tidal nonlinear advection. Nonlinear advection is found to result in an increased smoothing of the tidal structure when the QTDW is present in the MLT. When traveling planetary waves become large due to favorable propagation and amplification conditions, they can cause tidal variability either through nonlinear wave-wave interaction or through altering the background winds (Chang et al., 2011; Forbes and Moudden, 2012; Kumari and Oberheide, 2020; Liu, 2016; McCormack et al., 2009; Palo et al., 1999; Pancheva et al., 2006; Pedatella et al., 2016). 
It is known that the atmospheric layer between approximately 60 and $100 \mathrm{~km}$ can be treated as a transition region, where different physical mechanisms coexist (Kazimirovsky et al., 2003), such as gravity wave breaking and its associated eddy diffusion, shear instabilities, wave-mean, and wave-wave nonlinear interactions shaping the spatial structure of DW1 in this layer and its variations (e.g., short-term, seasonal, and interannual). The exact causes of the short-term tidal variability in the mesosphere and thermosphere have not been systematically studied. Since a complete tidal budget cannot be obtained from the observations, the best alternative is to examine simulations from a middle atmosphere general circulation model that follows a first-principles approach. Thus, employ the thermodynamic budget from eCMAM30 output (see Section 2.4) to study the physical mechanisms of the short-term DW1 variability. To our knowledge, no such analysis has been conducted to investigate the physical mechanisms of the short-term tidal variability of DW1 in the mesosphere and thermosphere. The results presented in the following sections will shed some light on our current understanding of the physical mechanisms of the short-term tidal variability of DW1.

\subsection{Possible Mechanisms in the MLT region}

A detailed description of the full thermodynamic equation can be found in Chapter 2. The forcing terms of the short-term tidal variability on the right hand side include adiabatic heating due to vertical motion, linear and nonlinear advection, and model physics such as short-wave heating and long-wave cooling, etc. The first step in the thermodynamic budget analysis of the short-term tidal variability is a comparison of the three possible terms (linear advection term: wave-mean flow interaction, nonlinear advection term: wave-wave interaction and short-wave heating) deduced from previous studies that cause the seasonal and interannual variability of DW1 (Chang

et al., 2012; Fomichev et al., 2002; Gu and Du, 2018; Lu et al., 2012; McLandress, 
2002a,b, 1997; Vincent, 2015). Short-term variability of the linear advection, nonlinear advection, and solar heating is examined to identify which terms have significant forcing magnitudes and have similar variations as the short-term variability of the tide.

\subsubsection{Roles of Linear, Nonlinear Advection, and Solar Heating in the MLT Region}

First examine the climatological mean of the forcing magnitudes and latitudinal structures in all three terms as shown in Figure 6.1. Figure 6.1 top left panel shows the climatological mean of DW1 heating rate (the left hand side of the thermodynamic equation: temperature time tendency) from January 1979 to June 2010 as a function of latitude $\left(45^{\circ} \mathrm{S}-45^{\circ} \mathrm{N}\right)$ and altitude $(55-100 \mathrm{~km})$. The temperature tendency of DW1 is simply $2 \pi$ times DW1 temperature amplitude. The mean structure of time tendency is characterized by an equatorial maximum and two secondary maxima located symmetrically in the midlatitudes (near $\pm 35^{\circ}$ ) of each hemisphere above $80 \mathrm{~km}$. The equatorial maximum is located between 95 and $100 \mathrm{~km}$ with amplitude of 110 - $120 \mathrm{~K} /$ day. The secondary maxima in the midlatitudes have maximum amplitudes of $50 \mathrm{~K} /$ day. The climatological mean of the linear advection (Figure 6.1 left middle panel) is maximized at the equator above $90 \mathrm{~km}$ with amplitudes of $\sim 60 \mathrm{~K} /$ day. The latitude-altitude structure of the linear advection around the equator is similar to the structure of the time tendency. It also shows secondary maxima at midlatitudes of each hemisphere at $100 \mathrm{~km}$ with amplitudes of $\sim 20 \mathrm{~K} /$ day. The latitudinal structure of nonlinear advection (Figure 6.1 left bottom panel) exhibits a broad feature extending from $45^{\circ} \mathrm{S}-45^{\circ} \mathrm{N}$ above $80 \mathrm{~km}$ and is symmetric around the equator. The symmetric maxima are located near $\pm 10^{\circ}$ in both hemispheres above $95 \mathrm{~km}$ with amplitudes of 40 to $50 \mathrm{~K} /$ day.

The solar heating (Figure 6.1 right top panel) for DW1 also exhibits a broad feature above $90 \mathrm{~km}$ and below $65 \mathrm{~km}$ from $45^{\circ} \mathrm{S}-45^{\circ} \mathrm{N}$. Solar heating decreases with 

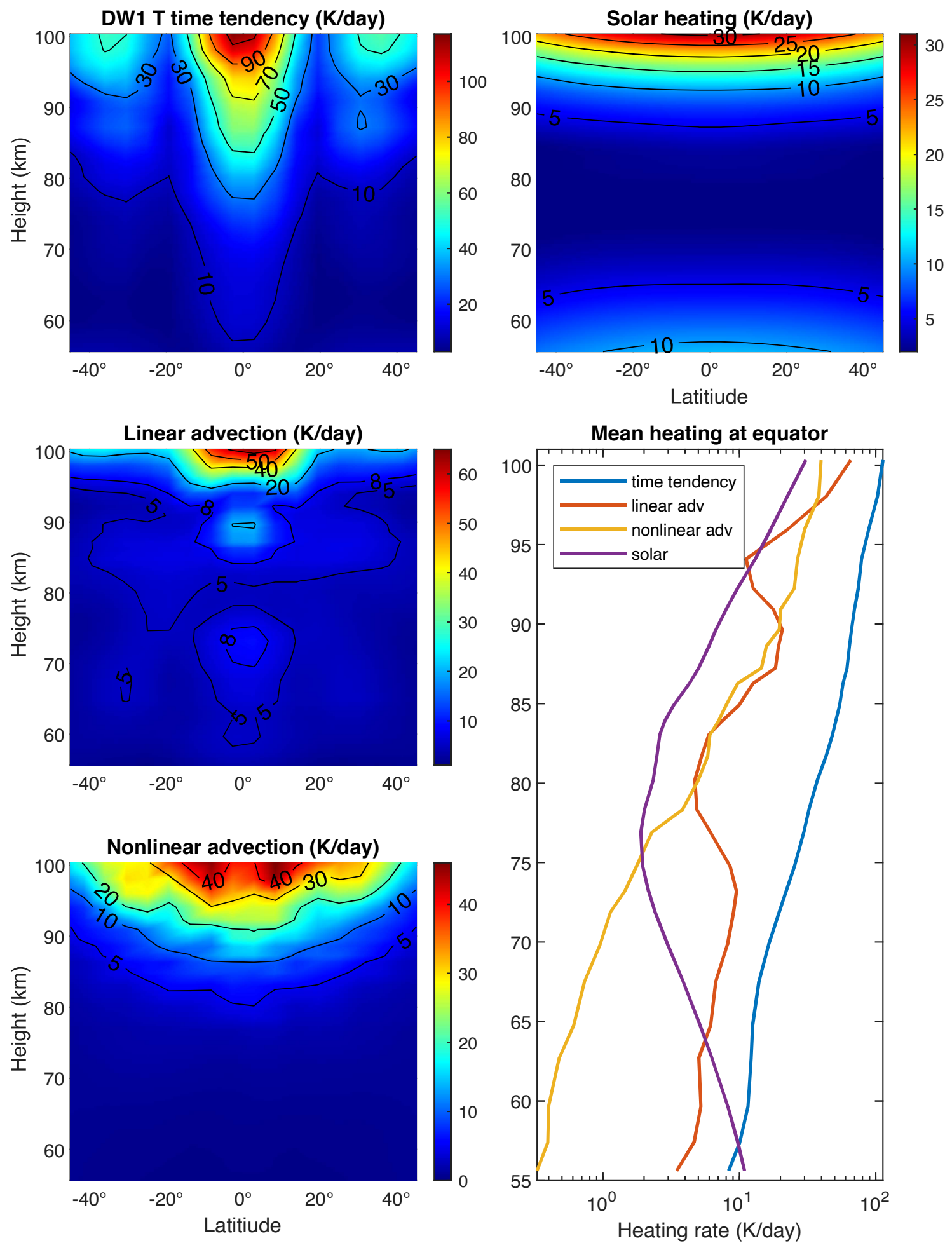

Figure 6.1. Climatological mean of DW1 T time tendency (left top), linear advection (left middle), nonlinear advection (left bottom), solar heating (right top), and average heating rates at the equator (right bottom). 
height from the stratopause $(\sim 55 \mathrm{~km})$ to $\sim 90 \mathrm{~km}$ and rapidly increases above $90 \mathrm{~km}$. Solar heating is minimum at $\sim 80 \mathrm{~km}$ due to very low Ozone $(<0.2 \mathrm{ppm})$ at this altitude (Smith et al., 2013). Vertical profiles of the climatologically averaged DW1 T time tendency and contributions from different mechanisms (linear advection, nonlinear advection, and solar heating) at the equator are shown in Figure 6.1 right bottom panel as a semi-logarithmic graph (logarithmic scale on the $x$-axis). The linear advection is dominated between $\sim 65 \mathrm{~km}$ to $\sim 80 \mathrm{~km}$ with a maximum of $\sim 10 \mathrm{~K} /$ day. Below $\sim 65 \mathrm{~km}$ solar heating is dominated mostly due to the absorption of solar energy by ozone in the Hartley bands. EUV heating starts to occur at $\sim 100 \mathrm{~km}$ and proliferates with height. Between $80 \mathrm{~km}$ to $90 \mathrm{~km}$, linear and nonlinear advection terms have similar heating rates.

To further analyze the causes of short-term tidal variability, the above heating budget terms were filtered to only keep variability less than 30-days e.g., short-term variability. Figure 6.2 presents the latitude-altitude structures of the standard deviations of the short-term variability in each term. It shows standard deviations of the DW1 T time tendency (top left panel), linear advection (top right panel), nonlinear advection (bottom left panel), and solar heating (bottom right panel), respectively. All the terms follow the latitude-altitude structure of their climatological mean in Figure 6.1. The maximum standard deviation for the short-term DW1 T time tendency is $\sim 20 \mathrm{~K} /$ day at the equator above $95 \mathrm{~km}$ and secondary maximas in midlatitudes have standard deviations of $10-14 \mathrm{~K} /$ day in both hemispheres. Linear advection also maximizes at the equator with maximum standard deviation of $15 \mathrm{~K} /$ day at $100 \mathrm{~km}$. Standard deviations of short-term linear advection exhibit variability up to $\sim 50 \%$ of its mean amplitude at $95 \mathrm{~km}$ and the equator. Nonlinear advection shows similar magnitude of standard deviations compared to the time tendency and it is maximized near $\pm 10^{\circ}$ in both hemispheres above $95 \mathrm{~km}$ with maximum standard deviation of $18 \mathrm{~K} /$ day. Nonlinear advection shows variability in standard deviations up 
Standard Deviation of Short-term Heating Rate
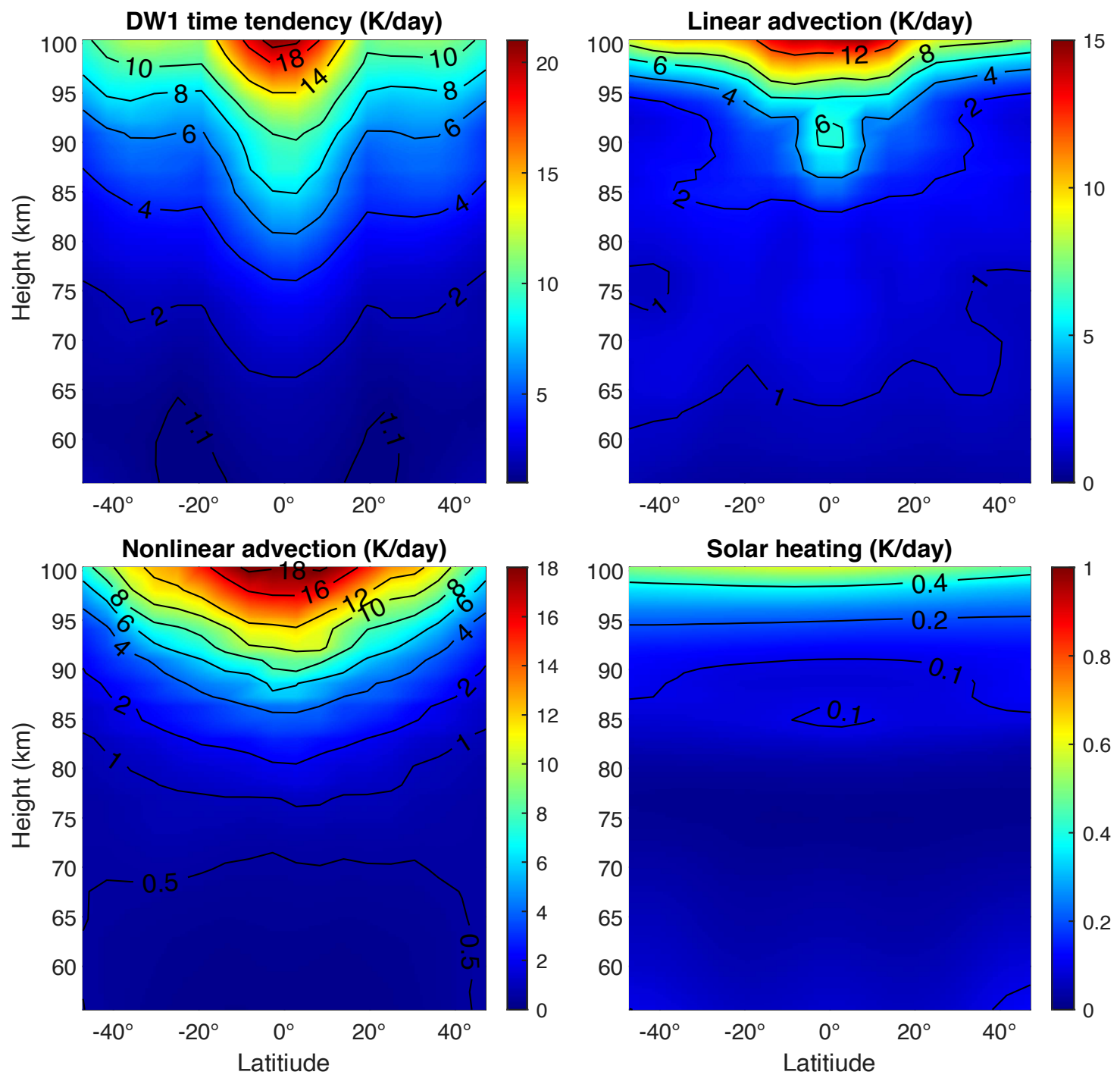

Figure 6.2. Standard deviations of short-term DW1 T time tendency (top left), linear advection (top right), nonlinear advection (bottom left), and solar heating (bottom right).

to $47 \%$ of its mean amplitude at $95 \mathrm{~km}$ and the equator.

Solar heating shows maximum standard deviation of $0.4 \mathrm{~K} /$ day from one hemisphere to another above $95 \mathrm{~km}$. In the mesosphere, variability of solar heating exhibits up to $2 \%$ of its mean amplitude. Compared to the standard deviations of the shortterm variability of linear and nonlinear advection, solar heating has a much smaller standard deviation in the mesosphere. Though the latitude structure of ozone has 
appreciable variations with season in the tropical upper mesosphere (Smith et al., 2013), Ozone may not change much on variability less than 30 days. This is likely the main reason for the smaller magnitude in the standard deviation. Comparison of the standard deviations from three terms at the equator shows that variability in the linear advection makes $40-75 \%$ of the composite standard deviation below $80 \mathrm{~km}$ and $30-40 \%$ above $80 \mathrm{~km}$. Below $80 \mathrm{~km}$ nonlinear variability is $20-55 \%$ and $50-65 \%$ above $80 \mathrm{~km}$. Solar heating is up to $10 \%$ below $60 \mathrm{~km}$ and up to $3 \%$ above $60 \mathrm{~km}$.

So far, we have examined the latitudinal structures of the climatological mean and standard deviation of the short-term variability from the three terms. Next, the shortterm variability time series will examined and look at the correlations between these forcing terms and the tidal response. Figure 6.3 shows the correlation between DW1 T time tendency and linear advection (top panel), nonlinear advection (middle panel), and solar heating (bottom panel) as a function of latitude and altitude for the period between 1979 and 2010. Linear advection exhibits strong correlations with the time tendency around the equator with maximum correlation $\sim 0.8$ between 60 and $80 \mathrm{~km}$, and the correlation decreases to 0.5 between 80 and $100 \mathrm{~km}$. Moderate correlation ( $0.3-\sim 0.4)$ is observed in the mid-latitudes of both hemispheres. Nonlinear advection and the time tendency are weakly correlated $(\sim 0.1)$ throughout the mesosphere. Correlation between solar heating and the time tendency is weakly correlated $(\sim 0.1)$ as well in the mesosphere. Though short-term variability of nonlinear advection and solar heating has a weak correlation with the short-term variability of tidal heating rate in the mesosphere, correlations calculated for the total tidal variability in these terms show strong correlations above $70 \mathrm{~km}$ (see Figure C1 in Appendix C). This implies that nonlinear advection and solar heating might be important in driving the long-term variability of the tide, they seem to be less important in governing the short-term tidal variability. 

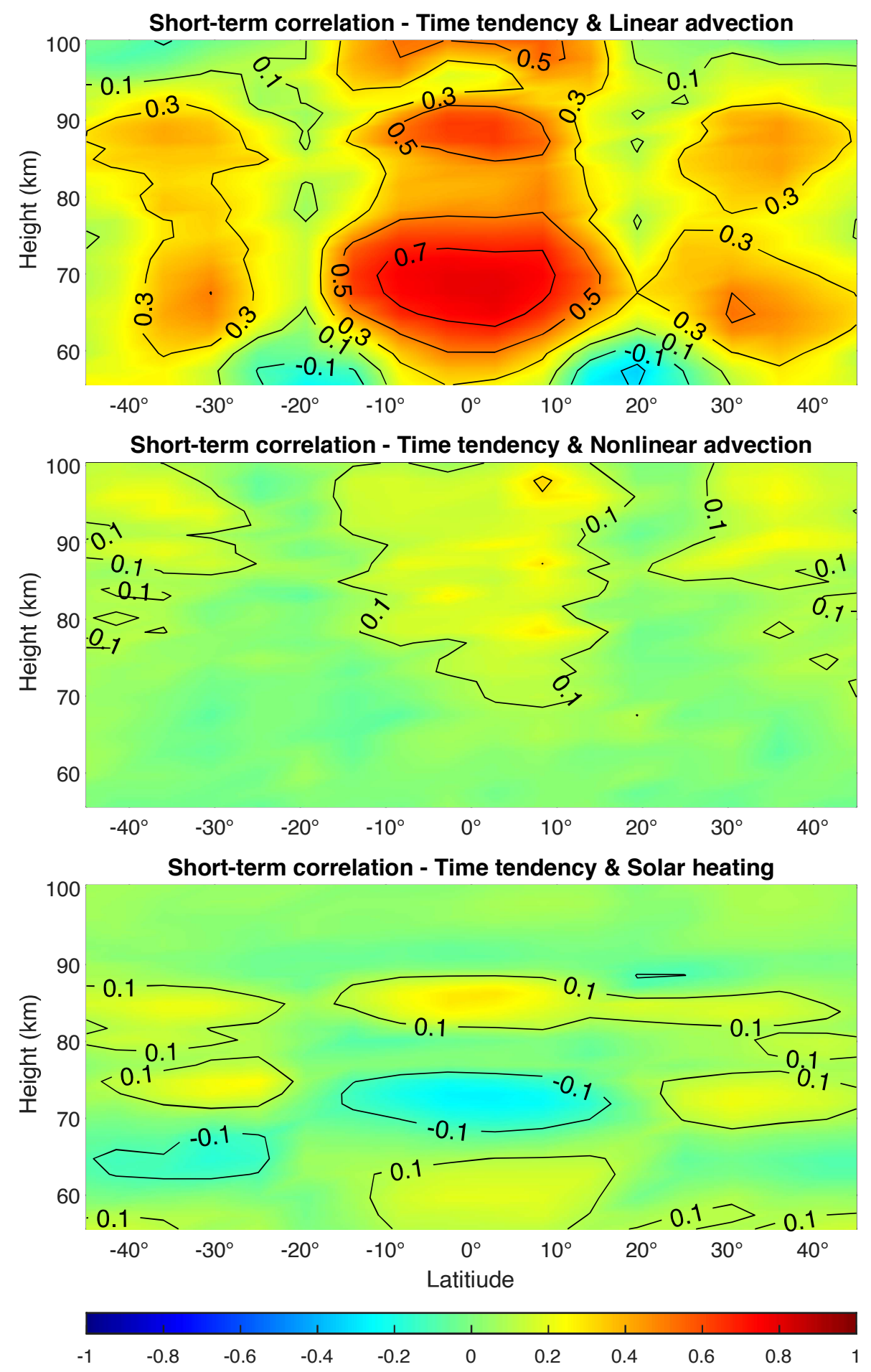

Figure 6.3. Short-term correlation between DW1 T time tendency and linear advection (top panel), nonlinear advection (middle panel), and solar heating (bottom panel) in the mesosphere as a function of latitude and height from the eCMAM30. 
Figure 6.4 illustrates the correlation between short-term DW1 T time tendency and linear advection at the equator as a function of altitude and year (from 1979 to 2009). Yearly correlations exhibit strong correlations $(0.7-0.9)$ between 60 and $80 \mathrm{~km}$ throughout the whole time period. The highest correlation at $70 \mathrm{~km}$ exhibits in years 1981-1984, 1986,1988, 1992-1996,1998-2000, and 2002-2005.

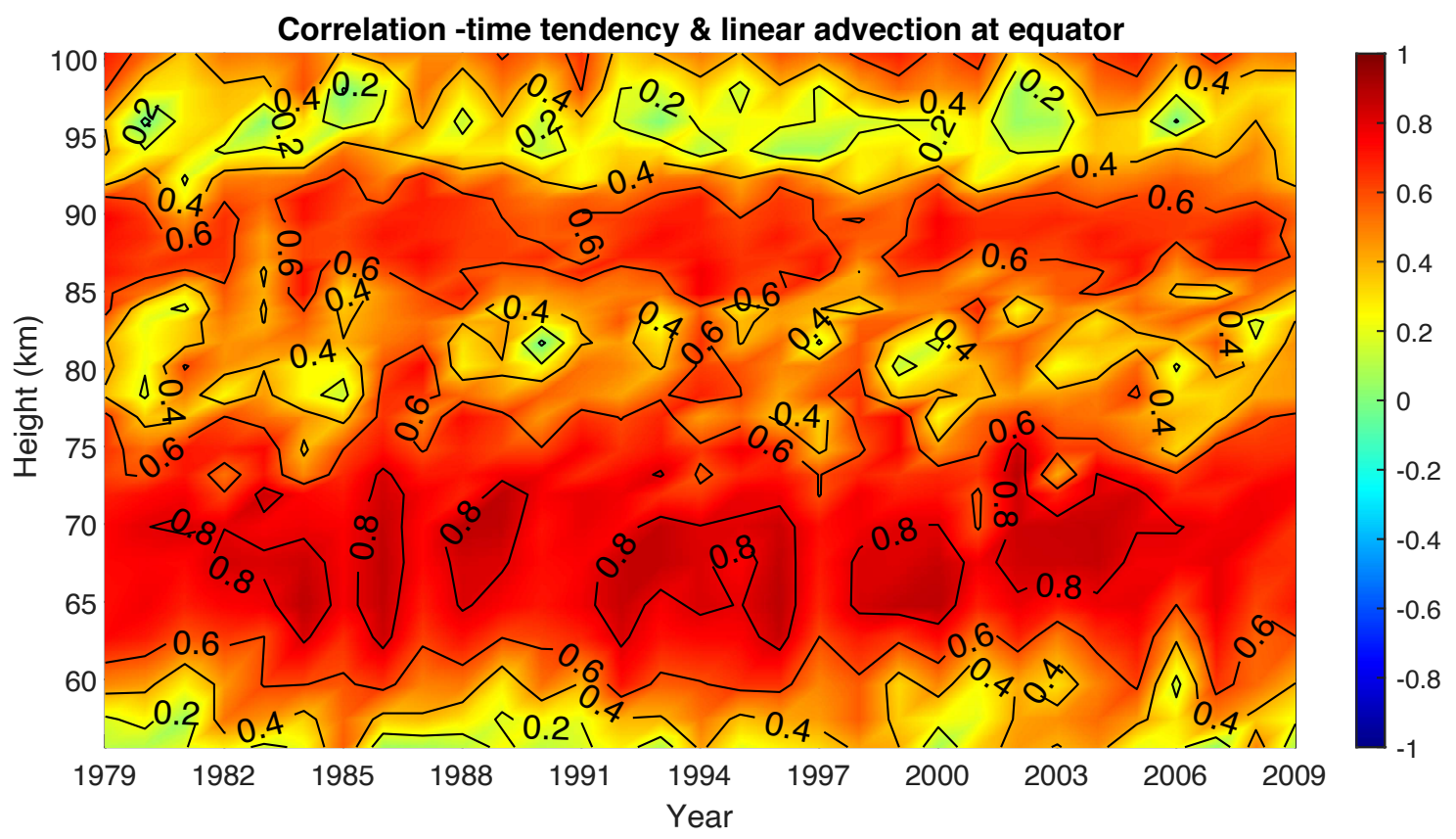

Figure 6.4. Yearly short-term correlation between DW1 T time tendency and linear advection at the equator from the eCMAM30.

Next, present an example of the short-term variability time series from the forcing terms and the response in 2004. Note that the short-term variability presented here is the anomaly from the climatological mean presented in Figure 6.1. Figure 6.5 presents the short-term variability of DW1 heating rate time series (first panel), shortterm variability of linear advection (second panel), short-term variability of nonlinear advection (third panel), and short-term variability of solar heating (fourth panel) from eCMAM at $70 \mathrm{~km}$ at the equator in 2004. Short-term linear advection time series is strongly correlated $(0.86)$ with the short-term time tendency time series and has the same order of magnitude. It is evident that there are similar periodic oscillations in 


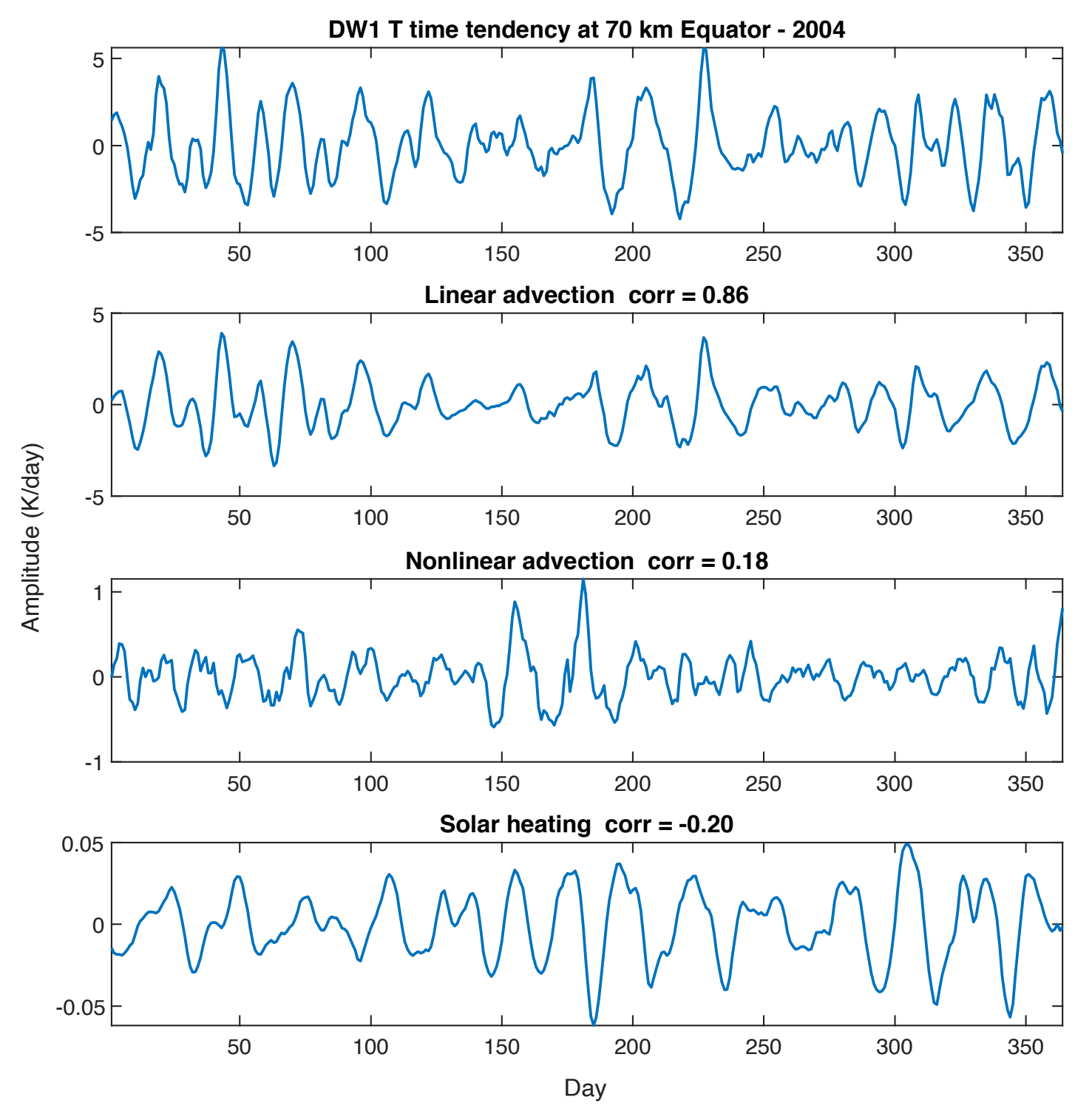

Figure 6.5. DW1 time tendency (first panel), linear advection (second panel), nonlinear advection (third panel), and solar heating at $70 \mathrm{~km}$ in the equator.

the short-term time tendency and short-term linear advection. Nonlinear advection is weakly correlated $(0.18)$ with the short-term time tendency and solar heating is weakly negatively correlated (-0.20) as well. Both the nonlinear advection and solar heating have negligible amplitudes compared to the magnitude of the time tendency at $70 \mathrm{~km}$. This implies that nonlinear advection and solar heating do not play an essential role in governing the short-term variation of the migrating diurnal tide at this altitude. 


\subsubsection{Role of Different Terms in the Linear Advection}

Analysis in the above section revealed that the correlation between the short-term linear advection and the short-term tidal variability is the strongest in the mesosphere compared to the nonlinear advection and solar heating forcing. To further examine the mechanisms behind the short-term tidal variability in the mesosphere, linear advection term is separated into five terms as presented in Equation 2.44:

$$
F_{\text {linearadvec }, T}=-\frac{\bar{u}}{a \cos \phi} \frac{\partial T^{\prime}}{\partial \lambda}-\frac{\bar{v}}{a} \frac{\partial T^{\prime}}{\partial \phi}-\frac{v^{\prime}}{a} \frac{\partial \bar{T}}{\partial \phi}-\bar{w} \frac{\partial T^{\prime}}{\partial z}-w^{\prime} \frac{\partial \bar{T}}{\partial z}
$$

The first term in the above equation is the product of zonal DW1 temperature gradient and zonal mean wind, second is the product of meridional DW1 temperature gradient and meridional mean wind, the third term is the product of meridional mean temperature gradient and DW1 meridional wind, the fourth term is the product of vertical DW1 temperature gradient and vertical mean wind, and the fifth term is the product of DW1 vertical wind and the vertical mean temperature gradient, also known as the lapse rate. The climatological mean of the above 5 terms is shown in Figure 6.6. The top left panel shows the climatological mean in time tendency as a function of latitude and height, same as the Figure 6.1 top left panel. Term one (top right panel) shows similar latitude-altitude structure as DW1. Term one is maximized at the equator with amplitudes of $3-4 \mathrm{~K} /$ day and two symmetrical secondary maxima at mid-latitudes (near $\pm 35^{\circ}$ ) of both hemispheres. The northern hemisphere's maximum is $\sim 1 \mathrm{~K} /$ day higher than the southern hemisphere. Term two (middle left panel) is maximized above $90 \mathrm{~km}$ and the maximum is located at $\sim \pm 10^{\circ}$ of both hemispheres and symmetric with the equator. The maximum amplitude is $\sim 6 \mathrm{~K} /$ day in the southern hemisphere compared to $\sim 4 \mathrm{~K} /$ day in the northern hemisphere. Term three (middle right panel) shows symmetrical latitude-altitude structure with respect to the equator. The maximum is located around $\pm 10^{\circ}$ of each hemisphere with amplitudes of $5-6 \mathrm{~K} /$ day. Term three also exhibits symmetric maxima with amplitudes 
of $2 \mathrm{~K}$ /day between $60-70 \mathrm{~km}$ in midlatitudes (near $\pm 40^{\circ}$ ).
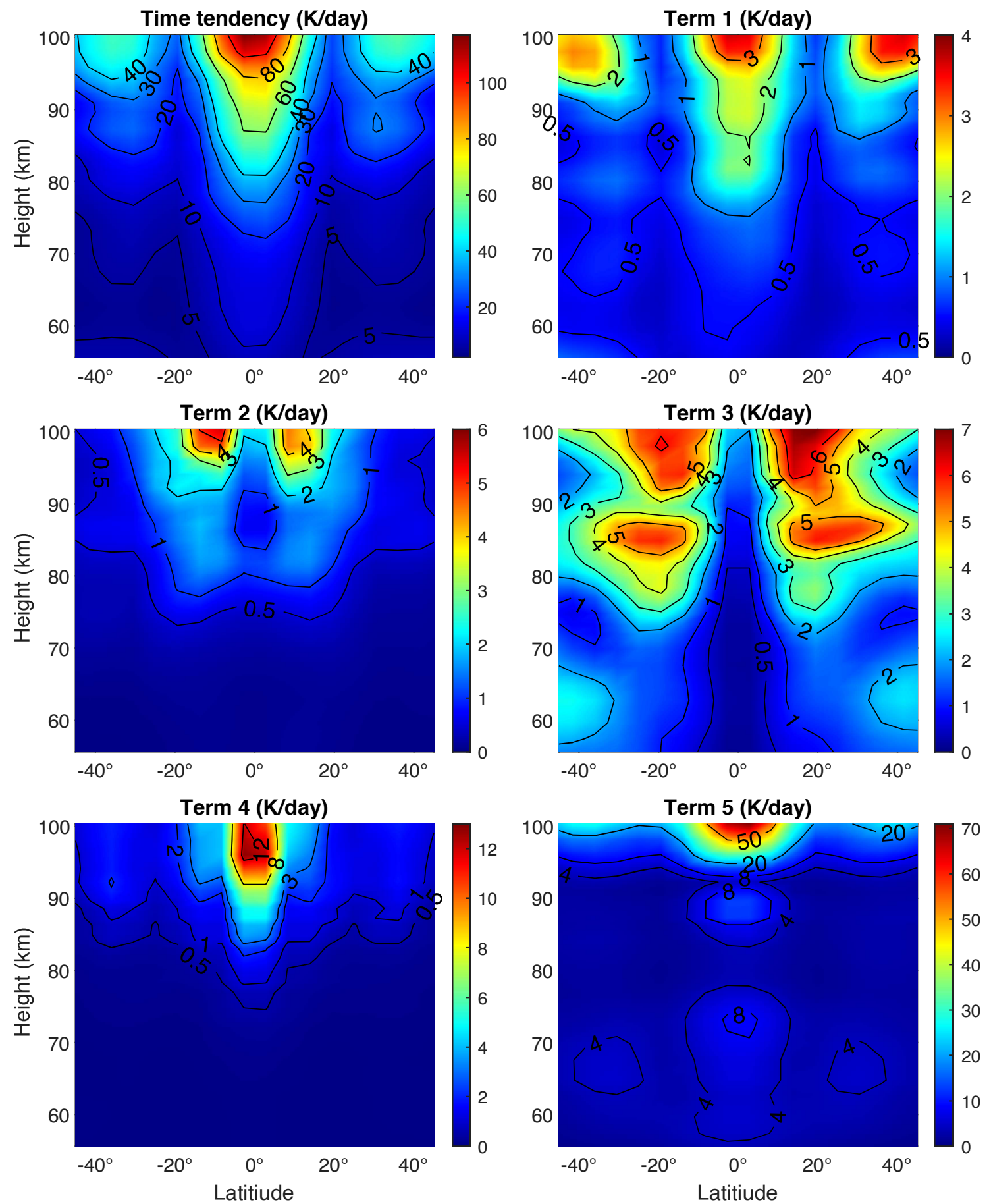

Figure 6.6. Climatological mean of time tendency (top left panel), linear advection term 1 (top right panel), term 2 (middle left panel), term 3 (middle right panel), term 4 (bottom left panel), and term 5 (bottom right panel) as a function of height and latitude. 
Term four (bottom left panel) is maximized above $90 \mathrm{~km}$ at the equator with amplitudes of $\sim 12 \mathrm{~K} /$ day. Term four exhibits similar latitude-altitude structure to the time tendency and term one, but with much weaker secondary maxima $\pm 35^{\circ}$ $\mathrm{N} / \mathrm{S}(1 \mathrm{~K} /$ day). Term five (bottom right panel) maximizes around the equator above $95 \mathrm{~km}$ and is the largest term among the five terms with amplitude of $70 \mathrm{~K} /$ day. A secondary maximum is located in each hemisphere near $35^{\circ}$ with amplitudes of $\sim 30 \mathrm{~K} /$ day at $100 \mathrm{~km}$. Term five and the time tendency are in the same order of magnitude and the other four terms are one magnitude smaller.

In the mesosphere, term five is the dominant term in the linear advection compared to the other four terms. Figure 6.7 shows the correlation between term five and DW1 $\mathrm{T}$ time tendency as a function of latitude and altitude for the period between 1979 and 2010 .

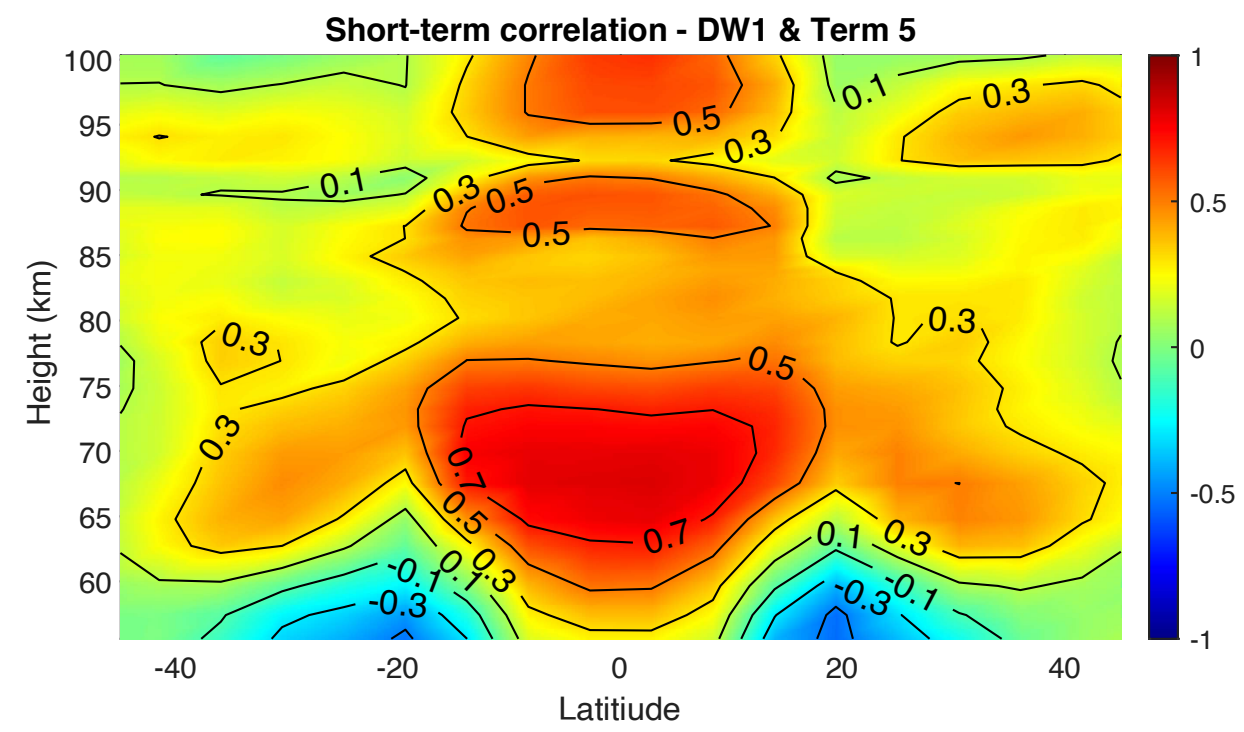

Figure 6.7. Short-term correlation between DW1 T time tendency and linear advection term five as a function of latitude and height from the eCMAM30.

Term five exhibits strong correlation with the time tendency around the equator with maximum correlation of $\sim 0.8$ between $60-80 \mathrm{~km}$ and moderate correlation $(\sim 0.3-\sim 0.5)$ above $80 \mathrm{~km}$. The correlation in the mid-latitudes $\left( \pm 35^{\circ}\right)$ is $\sim 0.3$ between $60-75 \mathrm{~km}$ and decreases with increasing height. The correlation shown here 
has very similar structures and magnitude as in Figure 6.3 top panel, which implies that the strong and positive correlation between short-term variability of the linear advection and DW1 can be mainly attributed to term five.

\subsubsection{Non-local Effect from Solar Heating}

The migrating diurnal tide DW1 is primarily exited by solar radiation absorption from

the tropospheric $\mathrm{H}_{2} \mathrm{O}$ and stratospheric ozone. Correlation analysis was performed to examine the non-local effect of solar heating from the stratosphere and troposphere on the short-term variability of the tide in the mesosphere. Figure 6.8 presents the correlation between the short-term variability of solar heating from all heights between 0 and $100 \mathrm{~km}$ with the short term tidal variability at $90 \mathrm{~km}$.

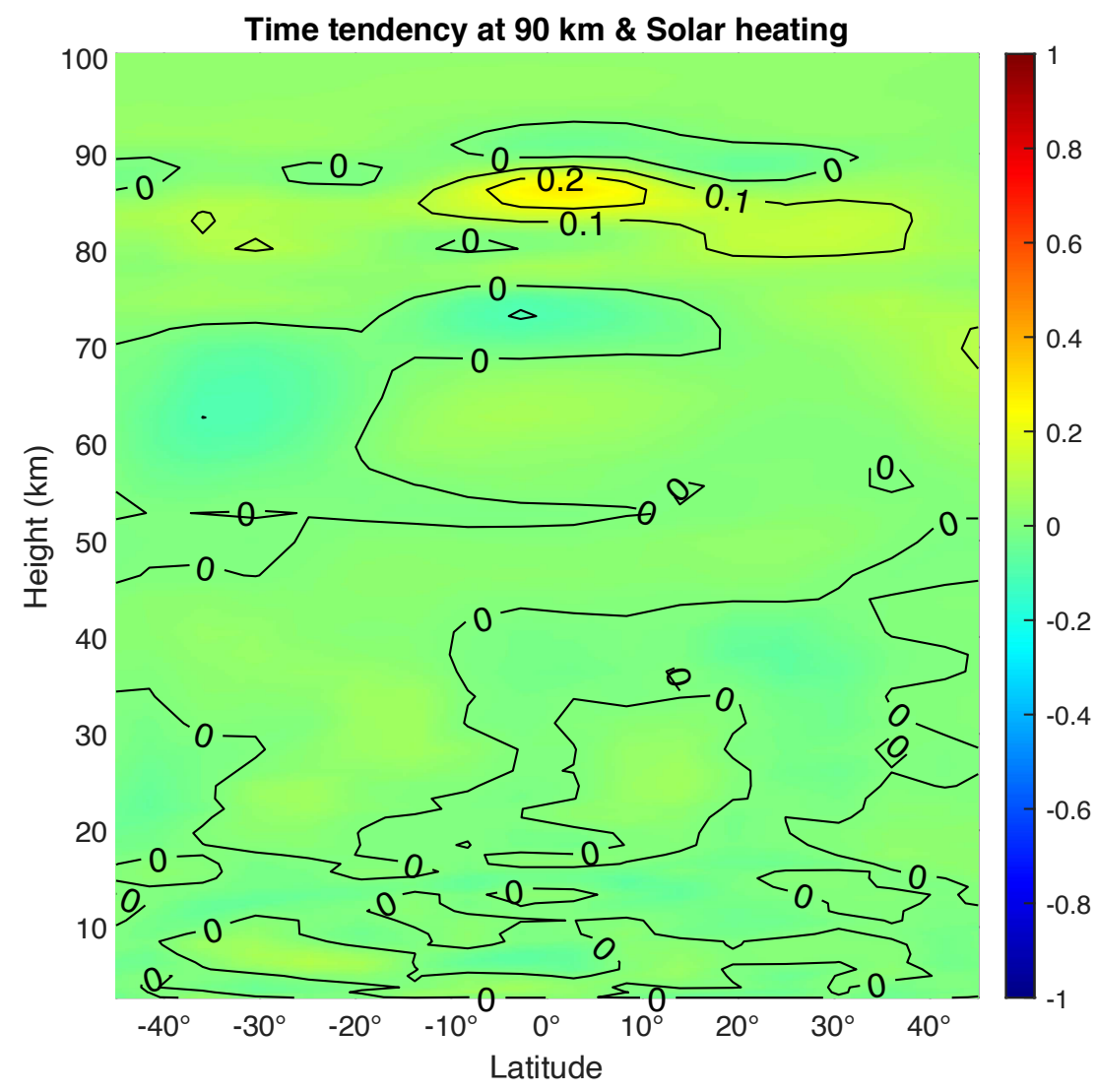

Figure 6.8. Correlations between short-term variability of solar heating at all heights with the short-term DW1 amplitude variability at $90 \mathrm{~km}$. 
The correlations at $90 \mathrm{~km}$ represent the local effect of solar heating, while they are non-local correlations at other heights. Figure 6.8 shows that the short-term variations in solar heating due to absorption of sunlight by water vapor in the troposphere and stratospheric ozone are weakly correlated (less than 0.1 ) with the short-term variations in DW1 amplitude at $90 \mathrm{~km}$. Although the total tidal variations at $90 \mathrm{~km}$ is well correlated with the solar heating below the stratosphere (see Figure C2 in Appendix C), the short-term variations of solar heating in the troposphere and stratosphere do not provide support for non-local effects being a possible cause for the short-term tidal variability in the mesosphere.

\subsection{Physical Mechanisms in Thermosphere}

DW1 is mainly generated in-situ by extreme ultraviolet (EUV) heating from atomic oxygen in the thermosphere (Forbes et al., 2011; Hagan et al., 2001). In this section, repeat the similar processes as did with the mesosphere and examine the physical mechanisms for the short-term tidal variability in the thermosphere. Solar heating, wave-wave interaction (nonlinear advection term), and mean-wave interaction (linear advection term) are deemed to be the plausible causes of short-term tidal variability from previous studies of the long-term tidal variability.

Figure 6.9 shows the 31.5-year climatological mean of DW1 time tendency (top left panel), linear advection (top right panel), nonlinear advection (bottom left panel), and solar heating (bottom right panel) in the thermosphere as a function of latitude and altitude $(110-230 \mathrm{~km})$. Note that the colorbar range for different terms is different. The latitudinal structure of DW1 $\mathrm{T}$ time tendency in the thermosphere is different from that in the mesosphere. It maximizes over a broad latitude region from one hemisphere to the other, with peak value ( $\sim 900 \mathrm{~K} /$ day) occurring in the equatorial region. Solar heating becomes the largest term in the thermosphere, with maximum amplitude up to $1300 \mathrm{~K} /$ day. The latitudinal structure of solar heating 
is very similar to that of the DW1 amplitude. Tidal heating increases rapidly with height above $100 \mathrm{~km}$ and is attributable to $\mathrm{O}_{2}$ absorption in the Schumann-Runge bands and continuum (Hagan, 1996). The EUV short-wave solar heating generates DW1 in-situ, which is trapped with a coherent phase throughout the thermosphere and the energy and momentum do not propagate vertically.
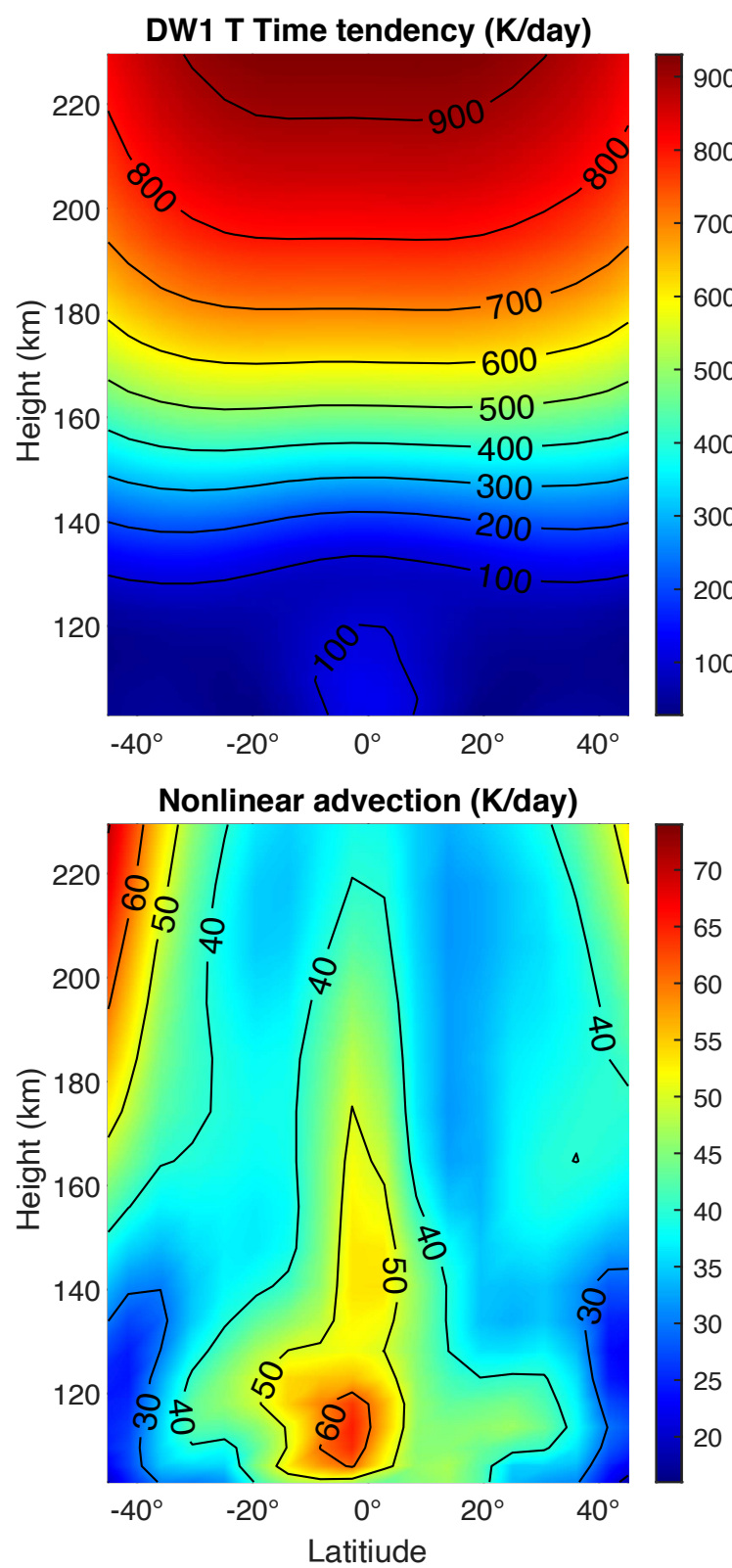
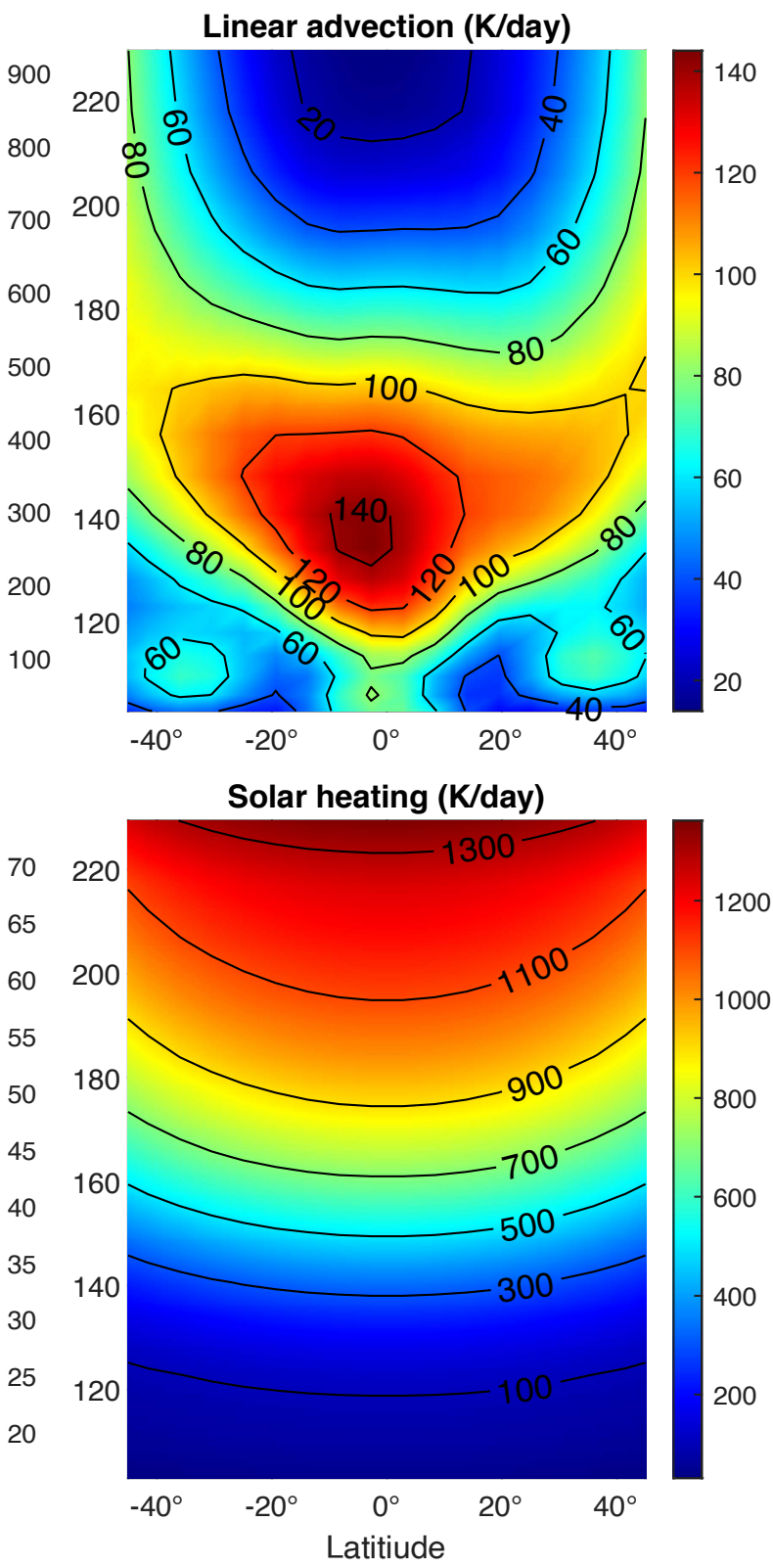

Figure 6.9. Climatological means of DW1 time tendency (top left panel), linear advection (top right panel), nonlinear advection (bottom left panel), and solar heating (bottom right panel). 
Linear advection is the second-largest term and maximizes $(140 \mathrm{~K} /$ day) at the equatorial region between $120-160 \mathrm{~km}$. Amplitudes in solar heating and linear advection are comparable below $140 \mathrm{~km}$. Linear advection amplitude decreases with the height above $160 \mathrm{~km}$ and reaches $20 \mathrm{~K} /$ day at $220 \mathrm{~km}$. Nonlinear advection maximizes at the equator below $120 \mathrm{~km}$ with amplitude of $\sim 60 \mathrm{~K} /$ day and decreases with height as well to $40 \mathrm{~K} /$ day at $220 \mathrm{~km}$. It is evident that the forcing amplitudes from linear advection, nonlinear advection, and solar heating are comparable in the lower thermosphere (below $140 \mathrm{~km}$ ) and solar heating begins to dominate the forcing above $140 \mathrm{~km}$.

Next, examine the correlations between the short-term variability of forcing amplitude from the three terms and the short-term DW1 amplitude in the thermosphere. Short-term variability of these time series is obtained to only keep variability period less than 30 days. Figure 6.10 shows the correlation between short-term variability of DW1 amplitude and the short-term variability of the three mechanisms as a function of latitude and altitude for the period between 1979 and 2010. The top panel shows the correlation between DW1 amplitude and linear advection. In the lower thermosphere (below $140 \mathrm{~km}$ ), the maximum correlations are between 0.3 and 0.5 . This is a region of transition between the mesosphere and thermosphere. As the linear advection term has the strongest effect in the mesosphere, its effect is still significant in the lower thermosphere. The forcing of the nonlinear advection is weakly correlated with the DW1 amplitude, which is similar to the mesosphere (middle panel). Figure 6.10 bottom panel shows the correlation between solar heating and DW1 amplitude on the short-term variability time scale. The correlation increases rapidly from 0.1 to 0.7 from 100 to $140 \mathrm{~km}$ and increases to 0.9 above $160 \mathrm{~km}$. Out of the proposed three mechanisms, solar heating strongly governs the DW1 in the thermosphere.

At thermospheric heights, solar heating mostly results from the absorption of EUV radiation by $\mathrm{O}_{2}, \mathrm{~N}_{2}$, and $\mathrm{O}$, followed by photoionization. In eCMAM, EUV heating 

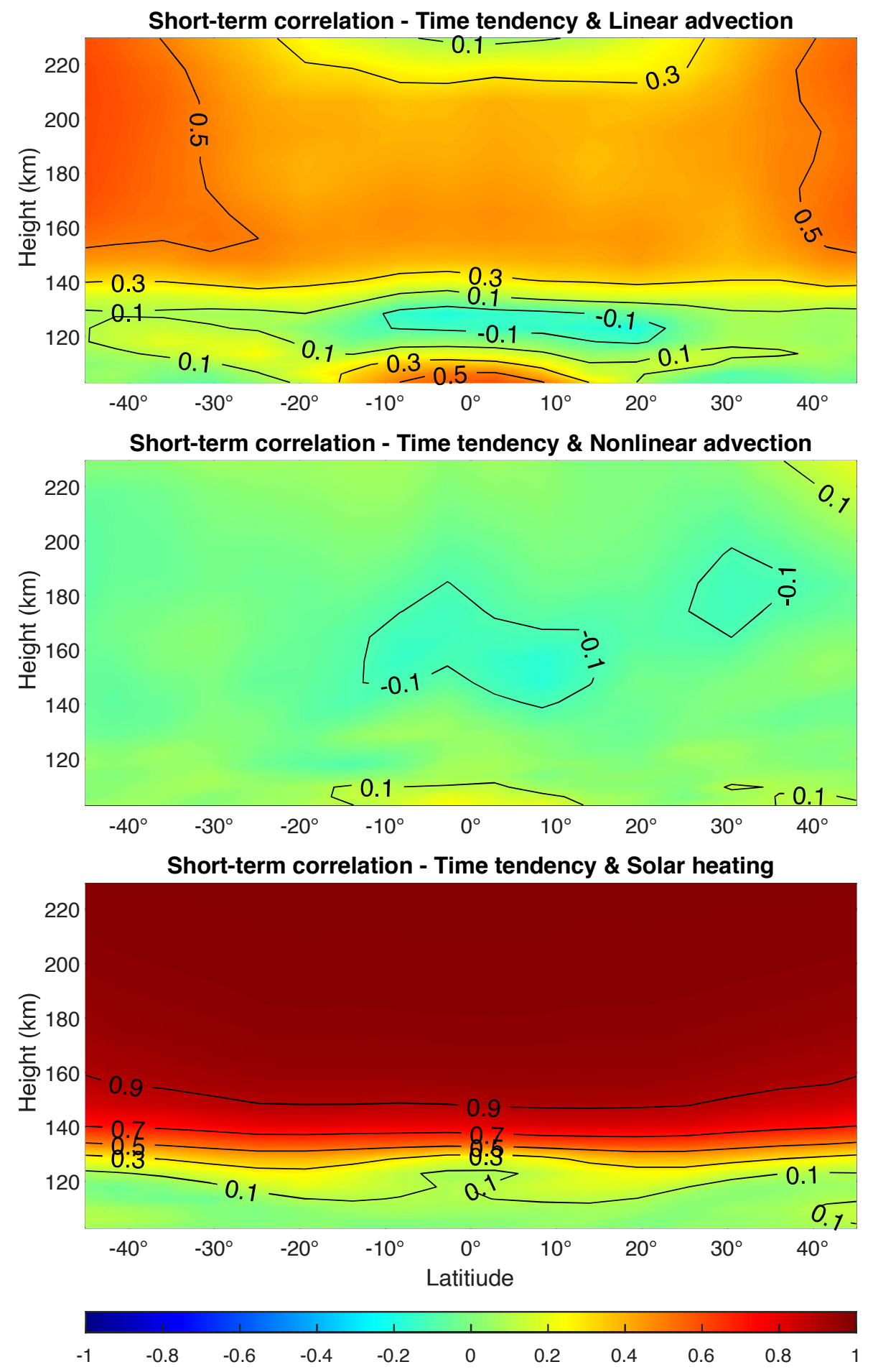

Figure 6.10. Short-term correlation between DW1 T time tendency and linear advection (top panel), nonlinear advection (middle panel), and solar heating (bottom panel) in the thermosphere. 
calculated by assuming moderate solar activity and does not consider thermospheric chemistry. Model does not include thermospheric processes such as joule heating and the heating associated with electromagnetic processes in polar regions and does not have an interactive ionosphere. Hence these conclusions are model specific. Further study in this section is needed using the next generation models such as WACCM-X.

Figure 6.11 presents the short-term variability of DW1 heating rate (tidal amplitude times $2 \pi$ ) and short-term variability of solar heating at $165 \mathrm{~km}$ and the equator in 2004 from eCMAM. Short-term solar heating (middle) is strongly correlated (0.98) with short-term DW1 heating rate (top) and has the same order of magnitude.
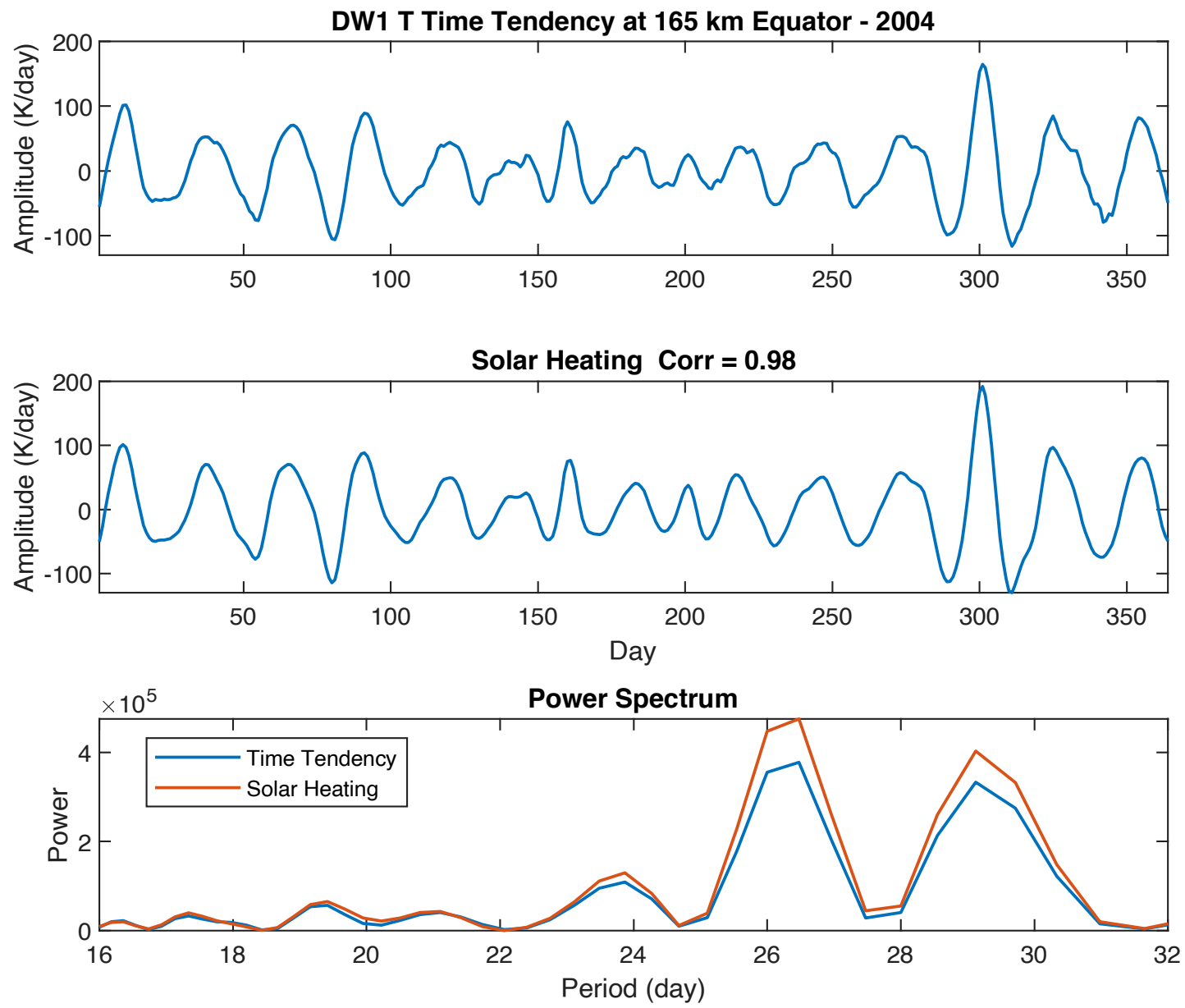

Figure 6.11. DW1 time tendency (top) and solar heating (middle) at $165 \mathrm{~km}$ in the equator and their power spectrum (bottom). 
It is clearly shown in the spectrum in the bottom panel that there are similar periodic oscillations ( $\sim 26$ and 29 days) in the short-term time tendency and short-term solar heating. Solar heating plays an essential role in governing the short-term variation of the migrating diurnal tide in the thermosphere.

\subsection{Conclusions}

The plausible causes for the short-term tidal variability are thought to be the variations in the background wind (wave-mean interaction), variability in the tidal sources (solar heating), and nonlinear interaction (wave-wave interaction). However, the exact causes for the short-term tidal variability in the mesosphere and thermosphere have not been systematically studied. The causes of the short-term tidal variability can be studied by investigating the momentum and thermodynamic budget terms of the tide. These budget terms are difficult to resolve from observations since multiple variables need to be obtained simultaneously. Hence the best way to obtain these budget terms are the use of general circulation models. This study uses the thermodynamic budget terms calculated from the 31.5-years $(1979-2010)$ run from the eCMAM and aims to shed some light on our current understanding of the short-term tidal variability. DW1 is known to have different latitude-altitude structures and seasonal variations in the stratosphere, mesosphere, and thermosphere. In this analysis, the roles of linear advection, nonlinear advection, and solar heating in the short-term variations of DW1 in the mesosphere and thermosphere are studied.

In the mesosphere, climatological mean amplitudes from the linear advection, nonlinear advection, and solar heating are in similar order of magnitude. However, if we filter the time series into long-term variation ( $>30$ days) and short-term variation ( $<30$ days), the short-term variability of the DW1 time tendency varies on the order of $20 \%$ (maximum standard deviation of $20 \mathrm{~K} /$ day) from its climatological mean (maximum of $100 \mathrm{~K} /$ day) at the equator. Analysis of the short-term variability of 
the above terms revealed linear advection and nonlinear advection exhibit $50 \%$ of the variability at the $95 \mathrm{~km}$ equator compared to their respective mean while solar heating exhibits $2 \%$ variability.

Correlations of the short-term temporal variability between the source terms and the DW1 amplitude are also examined. Linear advection term is strongly correlated ( $>0.8$ ) with the DW1 T time tendency in the mesosphere between 60 and $80 \mathrm{~km}$. Nonlinear advection and solar heating are weakly correlated $(\sim 0.1)$ with the shortterm variability of DW1 in the mesosphere. Gu and Du (2018) found that both linear and nonlinear advection terms play an important role in the seasonal variations of DW1 in the mesosphere. However, this study shows that short-term variations of DW1 in the mesosphere are mainly governed by the linear advection. The correlations between the linear advection and DW1 time tendency decrease to $0.3-0.5$ in the upper mesosphere. The dissipation term, e.g., vertical diffusion, starts to play a significant role in this region as the tide reaches its maximum amplitude (figure not shown).

The linear advection term is further studied by separating it into five terms. Terms 1, 4, and 5 have similar latitudinal structures as the DW1 structure (maximum at the equatorial region), while terms 2 and 3 maximize at the subtropics and their amplitude at the equator is negligible $(1-2 \mathrm{~K} /$ day $)$. Term 5 has the largest amplitudes in the equatorial region (maximum $50 \mathrm{~K}$ /day) compared to term 1 (maximum $4 \mathrm{~K} /$ day) and term 4 (maximum $10 \mathrm{~K} /$ day). Term 5 also presents strong correlations with the DW1 short-term variability. Overall, term five is the dominant term in the linear advection and is mostly responsible for the short-term variability in DW1 in the mesosphere $(60-80 \mathrm{~km})$.

Nonlinear interaction is a more complex process and there are likely multiple waves involved. Different waves maximize in different seasons and months. In this chapter, all the correlations are calculated with one year window, therefore, the correlation 
for nonlinear advection might be averaged out. In order to investigate this further, a smaller window is needed. Preliminary results from correlations calculated on monthly windows show that the nonlinear effect can also be significant for some months. This investigation is subject to future study.

The non-local effect of solar heating also investigated since DW1 is believed to be excited by the heating from water vapor in the troposphere and ozone in the stratosphere. The short-term variability of DW1 amplitude in the mesosphere is only weakly correlated $(\sim 0.1)$ with heating from the troposphere and stratosphere. Solar heating varies on a much smaller magnitude compared to its mean on the short-term basis than linear advection and nonlinear advection terms below mesosphere (Figures 6.1 and 6.2). Solar heating has been found to be an important factor governing the seasonal variation of DW1 (Gu and Du, 2018), however, it plays a negligible role in governing the short-term variability of DW1 in the mesosphere.

Physical mechanisms for the short-term DW1 variability are also investigated in the thermosphere $(100-220 \mathrm{~km})$. The lower thermosphere $(100-140 \mathrm{~km})$ acts as a transition region between mesosphere and thermosphere. Solar heating effect increases in this region, and linear advection also plays an important role. Above $140 \mathrm{~km}$, solar heating becomes the dominant term governing the variability of tides on the short-term basis. The correlation between the short-term variability of solar heating and DW1 amplitude is above 0.9 . Tidal amplitude variability tracks nearly perfectly with the solar heating variability and both of them present a quasi-27-day oscillation. Since budget terms cannot be obtained from observations, these findings are model specific. However, a detailed budget analysis such as this can provide insight into the physical mechanisms that govern the short-term variability of the tide in a model, given that the model can simulate the tide well when compared to the observations. 


\section{CHAPTER 7}

\section{CONCLUSIONS AND FUTURE WORKS}

The upper atmosphere, e.g., the thermosphere and ionosphere, is the transition layer from the Earth's atmosphere to space and is not only under the influence of the interplanetary space but also the lower atmospheric processes. The need to understand and predict the upper atmosphere has been increasing in recent years due to its potentially significant societal impacts such as communication, transportation, and national defence. A major science goal of this decade in the Atmosphere-IonosphereMagnetosphere Interactions (AIMI) in the decadal strategy survey for Solar and Space Physics $(2013-2022)$ is to "understand how tropospheric weather influences space weather." The variabilities (local-time, seasonal-latitudinal, day-to-day) of the thermosphere and ionosphere system owe much to the perturbations and forcing from the lower atmosphere during geomagnetic quiet and medium solar conditions. It is now recognized that the atmospheric waves (gravity waves, atmospheric tides, and planetary waves) play a key role in coupling the lower and upper atmosphere through modulation of thermospheric temperature and wind structure, influences on E region conductivities, generation of electric fields through dynamo action, and modification of thermospheric composition. In this dissertation, we focused our study on one of these three types of atmospheric waves: atmospheric tides. As these tides propagate upward from their source regions in the troposphere and stratosphere to the mesosphere and thermosphere, they interact with the background atmosphere and other waves 
and deposit their momentum and energy when they dissipate. Past studies of the atmospheric tides were limited to the climatological scale due to the lack of short-term tidal data from the satellite. This dissertation constitutes a study to make the step from "seasonal/climatological tides" to "tidal weather/short-term variability" (tidal variability $<30$-days). Particular attention was given to the short-term variability of migrating diurnal tide (DW1), the most significant and persistent tidal feature of the temperature and wind fields in the mesosphere and lower thermosphere (MLT) region. The data used in this dissertation come from a 30-year run (1979 - 2010) from the extended Canadian Middle Atmosphere Model (CMAM), a state of the art general circulation model that extends from the surface to about $210 \mathrm{~km}$, and temperature observations from Sounding of the Atmosphere using Broadband Emission Radiometry (SABER) onboard the TIMED satellite from 2002 to 2017. The short-term tidal variability is diagnosed from SABER using the tidal ascending-descending differencing method developed by Prof. Jens Oberheide of Clemson University (Oberheide et al., 2002).

The main body of this dissertation consists of three chapters (Chapter $4-6$ ). In Chapter 4, we investigated the statistical characteristics of short-term tidal variability (period $<30$ days), e.g., tidal weather. Based on these statistical properties, a hierarchy of statistical models, for example, the autoregressive (AR), vector AR (VAR), and parsimonious AR (PVAR) models, were developed to predict tidal weather. In Chapter 5, we focused on the total tidal variability, and investigated long-term tidal variability at the the interannual (periods $>12$ months), such as solar variability, ENSO, and QBO, and seasonal (periods $=3,4,6$ and 12 months) time scales with a multilinear regression model. We also examined scientific questions such as: is there any long-term modulation of the short-term tidal variability? and what is the relative importance of tidal variability at different time scales? At the end of Chapter 5 , a total tidal variability forecast model is developed by combining the multi-linear 
regression model and AR model from Chapter 4. Chapter 6 studied the possible physical mechanisms that govern the short-term tidal variability using the thermodynamics equation. In particular, we examined the linear advection (wave-mean flow interaction), nonlinear advection (wave-wave interaction), and short-wave solar heating terms in the thermodynamic budget in the mesosphere and thermosphere. Conclusions from the above three chapters are summarized below in Section 7.1. We end this chapter by our envision of future work in Section 7.2.

\subsection{Conclusions}

\subsubsection{Conclusions on Short-term Tidal Variability (Chapter 4)}

The models and observations have shown significant day-to-day variability of the tides for two decades. However, up to today, we have very little knowledge of it, e.g., how these variabilities evolve with time and space, is there any coherent temporal variability or is it totally stochastic? To better understand a complicated system as tidal weather (short-term tidal variability), we first need to find its statistical properties. We extract the DW1 short-term variability by applying a high-frequency filter of 30-days to the 31.5-years of tidal data derived from the eCMAM (1979 - 2010) run and the 7.5-years of tidal data derived from SABER (2003 - 2010) observations. Therefore, tidal weather refers to the tidal variability with periods $<30$ days. The autocorrelation of the tidal weather shows that there is a quasi-23-day periodicity in both eCMAM and SABER. Tidal weather is spatially strongly correlated with its neighboring latitudes and heights in both eCMAM and SABER. We utilized these statistical properties to build a simple AR model and two more complex models, e.g., VAR and PVAR. The simple AR model, which takes into account only the autocorrelation, has 23 coefficients at each location (latitude and height) and can predict

tidal weather to an accuracy of $89 \%$ at 1 -day. The accuracy is calculated as the $R^{2}$ values (correlation coefficients squared) between the predicted time series and the 
original time series. An accuracy of $89 \%$ means the correlation coefficient between the predicted and the original is 0.94 . The VAR and PVAR models, which take into account not only the auto-correlation but also the spatial correlations, slightly outperform the simple AR model but are much more computationally expensive with many more coefficients. The fact that the PVAR and VAR do not significantly improve forecast accuracy implies that the neighboring time series do not contain significantly more information than one time series. They are highly correlated spatially, most likely due to the common 23-day oscillation.

We performed a series of sensitivity tests on the AR model to make sure that the coefficients used in the model are robust. For example, we examined how many days of data are needed as input to have robust coefficients used in the statistical models, and found that 2000 days ( 5.5 years) would be more ideal. We also tested the model with coefficients calculated from two different 2000-day periods, e.g., $1979-1984$ and $2003-2008$, and found that the coefficients and the predictive accuracy are very similar.

A similar AR model was built based on tidal weather data from SABER. The coefficients of the AR model from SABER data agree very well with those from the eCMAM. The AR model with coefficients estimated from eCMAM performs nearly as well as that built on SABER. With the 23 coefficients and the prior 23-days of tidal weather data, we can predict the next day's tidal weather at near $90 \%$ accuracy (correlation coefficient: 0.95).

\subsubsection{Conclusions on Total Tidal Variability (Chapter 5)}

Wavelet analyses of the DW1 reveal that temporal variability of the tide has a rich spectrum at interannual, seasonal, and short-term time scales in both the eCMAM and SABER. Hence, we fit the data with a multi-linear regression model that contains deterministic variability such as the solar cycle, ENSO, QBO, and the seasonal 
harmonics at 12-, 6-, 4-, and 3- months. The fitting coefficients/amplitudes of each deterministic variability were examined. Both the model and SABER show that the DW1 amplitude is stronger in the solar maximum years than the solar minimum years. The DW1 amplitude is stronger during the cold-phase ENSO (La Niña) years than the warm-phase (El Niño) years. The DW1 T amplitude is stronger in the westerly phase of the QBO than in the easterly phase. For the seasonal harmonics in the equatorial region, both the model and SABER show that the 6-month harmonic has the strongest amplitude, and is followed by the 12-month harmonic. The 4- and 3-month harmonics are the smallest. The amplitudes follow a similar order for the mid-latitude maxima in the eCMAM.

One of the outstanding questions about short-term tidal variability is whether long-term variations in the atmosphere such as solar variability, ENSO, QBO, and seasonal variation can also modulate the short-term tidal variability. We investigate this question by examining both the absolute and relative variability of the shortterm tidal variability on a monthly basis. Absolute variability is the actual standard derivation of the time series over one month window, and relative variability is the absolute variability weighted by the monthly mean amplitude of the tide. Both absolute and relative variability of the short-term tidal time series show significant long-term variations. The 6 -month variation is the most prominent. We fitted a box plot to the absolute and relative variability from all March and June in the eCMAM and SABER. The results are rather surprising. The absolute variability shows that variability is larger in March when the mean tidal amplitude is stronger and smaller in June when the mean tidal amplitude is smaller. However, the relative variability shows an opposite picture. The relative variability is larger in June than March, which implies that short-term tidal amplitude varies more relative to its mean when the tidal mean amplitude is weaker. Since tides exhibit variability from daily to solar cycle time scales in the MLT region, it is crucial to assess the relative contribution 
of tidal variability at different time scales. Obviously, their relative contributions are a function of time length. We investigated these contributions at time lengths of 7 , 14, 21, 30, 60, 90, 120, 180 and 365 days. Short-term tidal variability contributes $\sim 50-75 \%$ of the total tidal variability between 7 to 30 days in SABER and eCMAM. SABER shows a higher amount of short-term variability compared to the eCMAM. Both the model and SABER agree that the seasonal variability (between 3 and 12 months) accounts for the largest variability $(70-90 \%)$ for time length greater than 60-days. The relative contribution from interannual tidal variability remains small and increases to less than $5 \%$ at time length of 365 days.

Total tidal variability can be forecasted using the coefficients obtained in the regression fit (long-term variability) and the tidal weather forecast from the AR model. For the total tidal forecast at the $95 \mathrm{~km}$ and the equator, eCMAM showed the highest $R^{2} \sim 0.97$ in 1990 and the lowest $R^{2} \sim 0.65$ in 2005 . The highest $R^{2}$ for the total tidal forecast in SABER is $\sim 0.85$ in 2011 and the lowest is $\sim 0.57$ in 2012. The relatively low $R^{2}$ for some years is mainly due to the regression model not capturing all the long-term tidal variability ( $>30$ days), since the short-term tidal forecast in both eCMAM and SABER have very high $R^{2}$ values $(\sim 0.9)$.

\subsubsection{Conclusions on the Physical Mechanisms of Short-term Tidal Variability (Chap- ter 6)}

The plausible causes for the short-term tidal variability are thought to be the variations in the background wind (wave-mean interaction), variability in the tidal sources (solar heating), and nonlinear interaction (wave-wave interaction). This study uses the thermodynamic budget terms calculated from the 31.5-years (1979 - 2010) run from the eCMAM and aims to shed some light on our current understanding of the short-term tidal variability. DW1 is known to have different latitude-altitude structures and seasonal variations in the stratosphere, mesosphere, and thermosphere. In 
this analysis, the roles of linear advection, nonlinear advection, and solar heating in the short-term variations of DW1 in the mesosphere and thermosphere are studied.

In the mesosphere, climatological mean amplitudes from the linear advection, nonlinear advection, and solar heating are in similar order of magnitude. However, if we filter the time series into long-term variation ( $>30$ days) and short-term variation $(<30$ days), the short-term variability of the DW1 time tendency varies on the order of $20 \%$ (maximum standard deviation of $20 \mathrm{~K} /$ day) from its climatological mean (maximum of $100 \mathrm{~K} /$ day) at the equator. Analysis of the short-term variability of the above terms revealed linear advection and nonlinear advection exhibit $\sim 50 \%$ of the variability at the $95 \mathrm{~km}$ equator compared to their respective mean while solar heating exhibits $2 \%$ variability.

Correlations of the short-term temporal variability between the source terms and the DW1 amplitude are also examined. Linear advection term is strongly correlated $(>0.8)$ with the DW1 T time tendency in the mesosphere between 60 and $80 \mathrm{~km}$. Nonlinear advection and solar heating are weakly correlated $(\sim 0.1)$ with the shortterm variability of DW1 in the mesosphere. Gu and Du (2018) found that both linear and nonlinear advection terms play an important role in the seasonal variations of DW1 in the mesosphere. However, this study shows that short-term variations of DW1 in the mesosphere are mainly governed by the linear advection. The correlations between the linear advection and DW1 time tendency decrease to $0.3-0.5$ in the upper mesosphere.

The linear advection term is further studied by separating it into five terms. Term 1 is the product of DW1 temperature zonal gradient and zonal mean wind, term 2 is the product of DW1 temperature meridional gradient and meridional mean wind, term 3 is the product of mean temperature meridional gradient and DW1 meridional wind, the term 4 is the product of DW1 temperature vertical gradient and vertical mean wind, and term 5 is the product of DW1 vertical wind and the mean temperature 
lapse rate. Terms 1, 4, and 5 have similar latitudinal structures as the DW1 structure (maximum at the equatorial region), while terms 2 and 3 maximize at the subtropics and their amplitude at the equator is negligible $(1-2 \mathrm{~K} /$ day $)$. Term 5 has the largest amplitudes in the equatorial region (maximum $50 \mathrm{~K} /$ day) compared to term 1 (maximum $4 \mathrm{~K} /$ day) and term 4 (maximum $10 \mathrm{~K} /$ day). Term 5 also presents strong correlations with the DW1 short-term variability. Overall, term 5 is the dominant term in the linear advection and is mostly responsible for the short-term variability in DW1 in the mesosphere $(60-80 \mathrm{~km})$.

DW1 is believed to be excited by the heating from water vapor in the troposphere and ozone in the stratosphere. Hence, we also investigated the non-local effect of solar heating. The short-term variability of DW1 amplitude in the mesosphere is only weakly correlated $(\sim 0.1)$ with heating from the troposphere and stratosphere. Short-term variability of solar heating varies on a very small magnitude compared to its mean $(\sim 2 \%)$ below $100 \mathrm{~km}$. Solar heating has been found to be an important factor governing the seasonal variation of DW1 (Gu and Du, 2018), however, it plays a negligible role in governing the short-term variability of DW1 in the mesosphere.

Physical mechanisms for the short-term DW1 variability are also investigated in the thermosphere $(100-220 \mathrm{~km})$. The lower thermosphere $(100-140 \mathrm{~km})$ acts as a transition region between mesosphere and thermosphere. Solar heating effect increases in this region, and linear advection also plays an important role. Above $140 \mathrm{~km}$, solar heating becomes the dominant term governing the variability of tides on the short-term basis. The correlation between the short-term variability of solar heating and DW1 amplitude is above 0.9. Tidal amplitude variability tracks nearly perfectly with the solar heating variability and both of them present a quasi-27-day oscillation. 


\subsection{Future Work}

Although this dissertation has made significant progress on our understanding of short-term tidal variability, especially for the migrating diurnal tide DW1 in the mesosphere and lower thermosphere (MLT) region, there are still many unanswered questions remaining. Future work on short-term tidal variability can be divided into the following areas:

1. Further understanding of the statistical properties of short-term tidal variability using different statistical methods/tools:

Kumari and Oberheide (2020) studied the inter-annual changes in the shortterm tidal variability of the non-migrating diurnal tide DE3 using an informationtheoretic approach based on Larson (2012). The key component of this framework is to formulate the time-dependent probability density functions (TDPDF) of the short-term tidal variability time series and assess the probability density function complexity and temporal variability using the information-theoretic measures, i.e. the Kullback-Leibler Divergence (Cover and Thomas, 2006).

2. Comparisons of the statistical properties of short-term tidal variability for different tidal components:

We found a quasi-23-day oscillation in the short-term variability of DW1. Is this oscillation present in other tidal components or is it only DW1 specific, or a combination of periods are present for other tidal components? This is still an open question. There has been very little literature on this topic. Miyoshi (2006) showed that fluctuations of the DE3 amplitude with periods of $20-60$ days are clearly seen in the GCM developed at Kyushu University. Fluctuations of the migrating semidiurnal tidal (SW2) amplitude with periods of $10-12$ and 25 days are found at altitude from 20 to $250 \mathrm{~km}$ in the same model (Miyoshi and Fujiwara, 2006). 
3. Extending the tidal weather forecast model to other tidal components (other than the DW1 component presented in this dissertation):

After identifying the statistical properties of the other tidal components, similar techniques from Chapter 4 can be applied to predict tidal weather of other tidal components. Depending on the simplicity of the statistical properties for a particular tidal component, the statistical models might be more complicated.

4. Further investigation on the Physics mechanisms of the short-term tidal variability:

Although this dissertation identified term 5 in the linear advection (wave-mean flow interaction) of the thermodynamic equation is most likely the source for the short-term tidal variability of DW1 in the mesosphere, the physical mechanisms of the quasi-23-day oscillation is still unknown.

5. Further study of the short-term tidal variability in the thermosphere and ionosphere:

This dissertation mainly focused on the short-term tidal variability in the mesosphere and lower thermosphere region. The eCMAM model used in this dissertation does not include an realistic ionosphere. How does the electrodynamics affect the short-term tidal variability is currently unknown. Further study in this area requires a model that has fully coupled neutral-ionosphere capability with a much higher upper lid, such as the WACCM-X (from the Earth surface to $\sim 500 \mathrm{~km})$. SABER measurement used in this work has a height limit of $95-110 \mathrm{~km}$. Satellite measurements beyond this altitude are needed to validate the model. We have to wait for GDC and DYNAMIC missions to fill this gap. 


\section{REFERENCES}

Akmaev, R. A. (2001). Seasonal variations of the terdiurnal tide in the mesosphere and lower thermosphere: A model study. Geophysical Research Letters, 28(19):3817-3820.

Akmaev, R. A., Fuller-Rowell, T. J., Wu, F., Forbes, J. M., Zhang, X., Anghel, A. F., Iredell, M. D., Moorthi, S., and Juang, H. M. (2008). Tidal variability in the lower thermosphere: Comparison of Whole Atmosphere Model (WAM) simulations with observations from TIMED. Geophysical Research Letters, 35(3):1-5.

Akmaev, R. A., Wu, F., Fuller-Rowell, T. J., and Wang, H. (2009). Midnight temperature maximum (MTM) in Whole Atmosphere Model (WAM) simulations. Geophysical Research Letters, 36(7):n/a-n/a.

Akmaev, R. A., Wu, F., Fuller-Rowell, T. J., Wang, H., and Iredell, M. D. (2010). Midnight density and temperature maxima, and thermospheric dynamics in Whole Atmosphere Model simulations. Journal of Geophysical Research: Space Physics, $115(\mathrm{~A} 8)$.

Altadill, D., Apostolov, E. M., Jacobi, C., and Mitchell, N. J. (2003). Six-day westward propagating wave in the maximum electron density of the ionosphere. Annales Geophysicae, 21(7):1577-1588.

Angelats i Coll, M. and Forbes, J. M. (2002). Nonlinear interactions in the upper atmosphere: The $\mathrm{s}=1$ and $\mathrm{s}=3$ nonmigrating semidiurnal tides. Journal of Geophysical Research: Space Physics, 107(A8):SIA 3-1-SIA 3-15.

Beagley, S., de Grandpré, J., Koshyk, J., McFarlane, N., and Shepherd, T. (1997). Radiative-dynamical climatology of the first-generation Canadian middle atmosphere model. Atmosphere-Ocean, 35(3):293-331.

Beagley, S. R., Boone, C. D., Fomichev, V. I., Jin, J. J., Semeniuk, K., McConnell, J. C., and Bernath, P. F. (2010). First multi-year occultation observations of CO 2 in the MLT by ACE satellite: observations and analysis using the extended CMAM. Atmospheric Chemistry and Physics, 10(3):1133-1153.

Beagley, S. R., McLandress, C., Fomichev, V. I., and Ward, W. E. (2000). The Extended Canadian Middle Atmosphere Model. Geophysical Research Letters, 27(16):2529-2532. 
Bilitza, D. and Reinisch, B. (2008). International Reference Ionosphere 2007: Improvements and new parameters. Advances in Space Research, 42(4):599-609.

Burrage, M. D., Hagan, M. E., Skinner, W. R., Wu, D. L., and Hays, P. B. (1995). Long-term variability in the solar diurnal tide observed by HRDI and simulated by the GSWM. Geophysical Research Letters, 22(19):2641-2644.

Cevolani, G. (1987). Tidal activity in the meteor zone over Budrio, Italy. In International Council of Scientific Unions, Middle Atmosphere Program, volume 25, pages 121-137.

Chang, J. L. and Avery, S. K. (1997). Observations of the diurnal tide in the mesosphere and lower thermosphere over Christmas Island. Journal of Geophysical Research: Atmospheres, 102(D2):1895-1907.

Chang, L., Ward, W., Palo, S., Du, J., Wang, D.-Y., Liu, H.-L., Hagan, M., Portnyagin, Y., Oberheide, J., Goncharenko, L., Nakamura, T., Hoffmann, P., Singer, W., Batista, P., Clemesha, B., Manson, A., Riggin, D., She, C.-Y., Tsuda, T., and Yuan, T. (2012). Comparison of diurnal tide in models and ground-based observations during the 2005 equinox CAWSES tidal campaign. Journal of Atmospheric and Solar-Terrestrial Physics, 78-79:19-30.

Chang, L. C., Palo, S. E., and Liu, H.-L. (2011). Short-term variability in the migrating diurnal tide caused by interactions with the quasi 2 day wave. Journal of Geophysical Research, 116(D12):D12112.

Chapman, S. and Lindzen, R. S. (1970). Atmospheric Tides. Springer Netherlands, Dordrecht.

Chau, J. L., Goncharenko, L. P., Fejer, B. G., and Liu, H.-L. (2012). Equatorial and Low Latitude Ionospheric Effects During Sudden Stratospheric Warming Events. Space Science Reviews, 168(1-4):385-417.

Cover, T. M. and Thomas, J. A. (2006). Elements of Information Theory (Wiley Series in Telecommunications and Signal Processing). Wiley-Interscience, USA.

Cullens, C. Y., Immel, T. J., Triplett, C. C., Wu, Y.-J., England, S. L., Forbes, J. M., and Liu, G. (2020). Sensitivity study for ICON tidal analysis. Progress in Earth and Planetary Science, 7(1):18.

Dawkins, E. C. M., Feofilov, A., Rezac, L., Kutepov, A. A., Janches, D., Höffner, J., Chu, X., Lu, X., Mlynczak, M. G., and Russell, J. (2018). Validation of SABER v2.0 Operational Temperature Data With Ground-Based Lidars in the MesosphereLower Thermosphere Region (75-105 km). Journal of Geophysical Research: Atmospheres, 123(17):9916-9934.

de Grandpré, J., Beagley, S. R., Fomichev, V. I., Griffioen, E., McConnell, J. C., Medvedev, A. S., and Shepherd, T. G. (2000). Ozone climatology using interactive 
chemistry: Results from the Canadian Middle Atmosphere Model. Journal of Geophysical Research: Atmospheres, 105(D21):26475-26491.

Dee, D. P., Uppala, S. M., Simmons, A. J., Berrisford, P., Poli, P., Kobayashi, S., Andrae, U., Balmaseda, M. A., Balsamo, G., Bauer, P., Bechtold, P., Beljaars, A. C. M., van de Berg, L., Bidlot, J., Bormann, N., Delsol, C., Dragani, R., Fuentes, M., Geer, A. J., Haimberger, L., Healy, S. B., Hersbach, H., Hólm, E. V., Isaksen, L., Kållberg, P., Köhler, M., Matricardi, M., McNally, A. P., Monge-Sanz, B. M., Morcrette, J.-J., Park, B.-K., Peubey, C., de Rosnay, P., Tavolato, C., Thépaut, J.-N., and Vitart, F. (2011). The ERA-Interim reanalysis: configuration and performance of the data assimilation system. Quarterly Journal of the Royal Meteorological Society, 137(656):553-597.

Dhadly, M. S., Emmert, J. T., Drob, D. P., McCormack, J. P., and Niciejewski, R. J. (2018). Short-Term and Interannual Variations of Migrating Diurnal and Semidiurnal Tides in the Mesosphere and Lower Thermosphere. Journal of Geophysical Research: Space Physics, 123(8):7106-7123.

Du, J. (2008). A mesosphere and lower thermosphere dynamics study using the extended Canadian Middle Atmosphere Model (CMAM). (Doctoral dissertation, University of New Brunswick, 2008). PhD thesis, University of New Brunswick.

Du, J., Ward, W., Oberheide, J., Nakamura, T., and Tsuda, T. (2007). Semidiurnal tides from the extended Canadian Middle Atmosphere Model (CMAM) and comparisons with TIMED Doppler interferometer (TIDI) and meteor radar observations. Journal of Atmospheric and Solar-Terrestrial Physics, 69(17-18):2159-2202.

Du, J. and Ward, W. E. (2010). Terdiurnal tide in the extended Canadian Middle Atmospheric Model (CMAM). Journal of Geophysical Research: Atmospheres, 115(D24).

Du, J., Ward, W. E., and Cooper, F. C. (2014). The character of polar tidal signatures in the extended Canadian Middle Atmosphere Model. Journal of Geophysical Research: Atmospheres, 119(10):5928-5948.

Eastes, R. W., McClintock, W. E., Burns, A. G., Anderson, D. N., Andersson, L., Codrescu, M., Correira, J. T., Daniell, R. E., England, S. L., Evans, J. S., Harvey, J., Krywonos, A., Lumpe, J. D., Richmond, A. D., Rusch, D. W., Siegmund, O., Solomon, S. C., Strickland, D. J., Woods, T. N., Aksnes, A., Budzien, S. A., Dymond, K. F., Eparvier, F. G., Martinis, C. R., and Oberheide, J. (2017). The Global-Scale Observations of the Limb and Disk (GOLD) Mission. Space Science Reviews, 212(1-2):383-408.

Eastwood, J. P. (2008). The science of space weather. Philosophical Transactions of the Royal Society A: Mathematical, Physical and Engineering Sciences, 366(1884):4489-4500. 
Eckermann, S. D., Rajopadhyaya, D. K., and Vincent, R. A. (1997). Intraseasonal wind variability in the equatorial mesosphere and lower thermosphere: long-term observations from the central Pacific. Journal of Atmospheric and Solar-Terrestrial Physics, 59(6):603-627.

Ekanayake, E. M. P., Aso, T., and Miyahara, S. (1997). Background wind effect on propagation of nonmigrating diurnal tides in the middle atmosphere. Journal of Atmospheric and Solar-Terrestrial Physics, 59(4):401-429.

England, S. L., Immel, T. J., Sagawa, E., Henderson, S. B., Hagan, M. E., Mende, S. B., Frey, H. U., Swenson, C. M., and Paxton, L. J. (2006a). Effect of atmospheric tides on the morphology of the quiet time, postsunset equatorial ionospheric anomaly. Journal of Geophysical Research, 111(A10).

England, S. L., Maus, S., Immel, T. J., and Mende, S. B. (2006b). Longitudinal variation of the E-region electric fields caused by atmospheric tides. Geophysical Research Letters, 33(21).

Fang, T.-W., Akmaev, R., Fuller-Rowell, T., Wu, F., Maruyama, N., and Millward, G. (2013). Longitudinal and day-to-day variability in the ionosphere from lower atmosphere tidal forcing. Geophysical Research Letters, 40(11):2523-2528.

Fernández, N. C., Herrera, R. G., Puyol, D. G., MartÍn, E. H., GarcÍa, R. R., Presa, L. G., and RodrÍguez, P. R. (2004). Analysis of the ENSO Signal in Tropospheric and Stratospheric Temperatures Observed by MSU, 1979-2000. Journal of Climate, 17(20):3934-3946.

Fesen, C. G. (1996). Simulations of the low-latitude midnight temperature maximum. Journal of Geophysical Research: Space Physics, 101(A12):26863-26874.

Fomichev, V. I., Ward, W. E., Beagley, S. R., McLandress, C., McConnell, J. C., McFarlane, N. A., and Shepherd, T. G. (2002). Extended Canadian Middle Atmosphere Model: Zonal-mean climatology and physical parameterizations. Journal of Geophysical Research: Atmospheres, 107(D10):ACL 9-1-ACL 9-14.

Fong, W., Lu, X., Chu, X., Fuller-Rowell, T. J., Yu, Z., Roberts, B. R., Chen, C., Gardner, C. S., and McDonald, A. J. (2014). Winter temperature tides from 30 to $110 \mathrm{~km}$ at McMurdo $\left(77.8^{\circ} \mathrm{S}, 166.7^{\circ} \mathrm{E}\right)$, Antarctica: Lidar observations and comparisons with WAM. Journal of Geophysical Research: Atmospheres, 119(6):28462863.

Forbes, J. and Vial, F. (1989). Monthly simulations of the solar semidiurnal tide in the mesosphere and lower thermosphere. Journal of Atmospheric and Terrestrial Physics, 51(7-8):649-661.

Forbes, J. M. (1982a). Atmospheric tide: 2. The solar and lunar semidiurnal components. Journal of Geophysical Research: Space Physics, 87(A7):5241-5252. 
Forbes, J. M. (1982b). Atmospheric tides: 1. Model description and results for the solar diurnal component. Journal of Geophysical Research: Space Physics, 87(A7):5222-5240.

Forbes, J. M. (1995). Tidal and Planetary Waves. The Upper Mesosphere and Lower Thermosphere: A Review of Experiment and Theory, Geophys. Monogr. Ser, 87:6787.

Forbes, J. M. (2003). Nonmigrating diurnal tides in the thermosphere. Journal of Geophysical Research, 108(A1):1033.

Forbes, J. M. and Garrett, H. B. (1976). SOLAR DIURNAL TIDE IN THE THERMOSPHERE. Journal of the Atmospheric Sciences, 33(11):2226-2241.

Forbes, J. M. and Garrett, H. B. (1979). Theoretical studies of atmospheric tides. Reviews of Geophysics, 17(8):1951.

Forbes, J. M., Hagan, M. E., Zhang, X., and Hamilton, K. (1997). Upper atmosphere tidal oscillations due to latent heat release in the tropical troposphere. Annales Geophysicae, 15(9):1165-1175.

Forbes, J. M., Makarov, N. A., and Portnyagin, Y. I. (1995). First results from the meteor radar at South Pole: A large 12-hour oscillation with zonal wavenumber one. Geophysical Research Letters, 22(23):3247-3250.

Forbes, J. M. and Moudden, Y. (2012). Quasi-two-day wave-tide interactions as revealed in satellite observations. Journal of Geophysical Research: Atmospheres, $117(\mathrm{D} 12)$.

Forbes, J. M., Palo, S. E., and Zhang, X. (2000). Variability of the ionosphere. Journal of Atmospheric and Solar-Terrestrial Physics, 62(8):685-693.

Forbes, J. M., Russell, J., Miyahara, S., Zhang, X., Palo, S., Mlynczak, M., Mertens, C. J., and Hagan, M. E. (2006). Troposphere-thermosphere tidal coupling as measured by the SABER instrument on TIMED during July-September 2002. Journal of Geophysical Research, 111(A10).

Forbes, J. M. and Wu, D. (2006). Solar Tides as Revealed by Measurements of Mesosphere Temperature by the MLS Experiment on UARS. Journal of the Atmospheric Sciences, 63(7):1776-1797.

Forbes, J. M., Zhang, X., Bruinsma, S., and Oberheide, J. (2011). Sun-synchronous thermal tides in exosphere temperature from CHAMP and GRACE accelerometer measurements. Journal of Geophysical Research: Space Physics, 116(A11):n/a$\mathrm{n} / \mathrm{a}$.

Forbes, J. M., Zhang, X., and Hagan, M. E. (2001). Simulations of diurnal tides due to tropospheric heating from the NCEP/NCAR Reanalysis Project. Geophysical Research Letters, 28(20):3851-3854. 
Forbes, J. M., Zhang, X., Palo, S., Russell, J., Mertens, C. J., and Mlynczak, M. (2008). Tidal variability in the ionospheric dynamo region. Journal of Geophysical Research: Space Physics, 113(A2).

Ford, E. A. K., Aruliah, A. L., Griffin, E. M., and McWhirter, I. (2006). Thermospheric gravity waves in Fabry-Perot Interferometer measurements of the 630.0nm OI line. Annales Geophysicae, 24(2):555-566.

Forster, P. M., Fomichev, V. I., Rozanov, E., Cagnazzo, C., Jonsson, A. I., Langematz, U., Fomin, B., Iacono, M. J., Mayer, B., Mlawer, E., Myhre, G., Portmann, R. W., Akiyoshi, H., Falaleeva, V., Gillett, N., Karpechko, A., Li, J., Lemennais, P., Morgenstern, O., Oberländer, S., Sigmond, M., and Shibata, K. (2011). Evaluation of radiation scheme performance within chemistry climate models. Journal of Geophysical Research, 116(D10):D10302.

Fricke-Begemann, C. and Höffner, J. (2005). Temperature tides and waves near the mesopause from lidar observations at two latitudes. Journal of Geophysical Research D: Atmospheres, 110(19):1-12.

Fritts, D. C. and Isler, J. R. (1994). Mean Motions and Tidal and Two-Day Structure and Variability in the Mesosphere and Lower Thermosphere over Hawaii. Journal of the Atmospheric Sciences, 51(14):2145-2164.

Fritts, D. C., Janches, D., Iimura, H., Hocking, W. K., Mitchell, N. J., Stockwell, R. G., Fuller, B., Vandepeer, B., Hormaechea, J., Brunini, C., and Levato, H. (2010). Southern Argentina Agile Meteor Radar: System design and initial measurements of large-scale winds and tides. Journal of Geophysical Research, 115(D18).

Fujiwara, H., Miyoshi, Y., Jin, H., Shinagawa, H., Otsuka, Y., Saito, A., and Ishii, M. (2009). Thermospheric temperature and density variations. Proceedings of the International Astronomical Union, 5(S264):310-319.

Fuller-Rowell, T., Wu, F., Akmaev, R., Fang, T.-W., and Araujo-Pradere, E. (2010). A whole atmosphere model simulation of the impact of a sudden stratospheric warming on thermosphere dynamics and electrodynamics. Journal of Geophysical Research: Space Physics, 115(A10).

Fuller-Rowell, T. J., Fang, T.-W., Wang, H., Matthias, V., Hoffmann, P., Hocke, K., and Studer, S. (2016). Impact of Migrating Tides on Electrodynamics During the January 2009 Sudden Stratospheric Warming, chapter 14, pages 163-174. American Geophysical Union (AGU).

Gan, Q., Du, J., Ward, W. E., Beagley, S. R., Fomichev, V. I., and Zhang, S. (2014). Climatology of the diurnal tides from eCMAM30 (1979 to 2010) and its comparison with SABER. Earth, Planets and Space, 66(1):103. 
Gasperini, F., Hagan, M. E., and Zhao, Y. (2017). Evidence of Tropospheric 90 Day Oscillations in the Thermosphere. Geophysical Research Letters, 44(20):10,12510,133 .

Glass, M. and Fellous, J. L. (1975). The eight-hourly (terdiurnal) component of atmospheric tides. Space Research, 15:191-197.

Goncharenko, L. and Zhang, S.-R. (2008). Ionospheric signatures of sudden stratospheric warming: Ion temperature at middle latitude. Geophysical Research Letters, $35(21)$.

Goncharenko, L. P., Coster, A. J., Plumb, R. A., and Domeisen, D. I. V. (2012). The potential role of stratospheric ozone in the stratosphere-ionosphere coupling during stratospheric warmings. Geophysical Research Letters, 39(8).

Grieger, N., Schmitz, G., and Achatz, U. (2004). The dependence of the nonmigrating diurnal tide in the mesosphere and lower thermosphere on stationary planetary waves. Journal of Atmospheric and Solar-Terrestrial Physics, 66(6-9):733-754.

$\mathrm{Gu}, \mathrm{H}$. and $\mathrm{Du}, \mathrm{J}$. (2018). On the Roles of Advection and Solar Heating in Seasonal Variation of the Migrating Diurnal Tide in the Stratosphere, Mesosphere, and Lower Thermosphere. Atmosphere, 9(11):440.

Gurubaran, S. (2005). Interannual variability of diurnal tide in the tropical mesopause region: A signature of the El Nino-Southern Oscillation (ENSO). Geophysical Research Letters, 32(13).

Hagan, M. E. (1996). Comparative effects of migrating solar sources on tidal signatures in the middle and upper atmosphere. Journal of Geophysical Research: Atmospheres, 101(D16):21213-21222.

Hagan, M. E., Burrage, M. D., Forbes, J. M., Hackney, J., Randel, W. J., and Zhang, X. (1999a). GSWM-98: Results for migrating solar tides. Journal of Geophysical Research: Space Physics, 104(A4):6813-6827.

Hagan, M. E., Burrage, M. D., Forbes, J. M., Hackney, J., Randel, W. J., and Zhang, X. (1999b). QBO effects on the diurnal tide in the upper atmosphere. Earth, Planets and Space, 51(7-8):571-578.

Hagan, M. E. and Forbes, J. M. (2002). Migrating and nonmigrating diurnal tides in the middle and upper atmosphere excited by tropospheric latent heat release. Journal of Geophysical Research: Atmospheres, 107(D24).

Hagan, M. E. and Forbes, J. M. (2003). Migrating and nonmigrating semidiurnal tides in the upper atmosphere excited by tropospheric latent heat release. Journal of Geophysical Research: Space Physics, 108(A2).

Hagan, M. E., Forbes, J. M., and Vial, F. (1995). On modeling migrating solar tides. Geophysical Research Letters, 22(8):893-896. 
Hagan, M. E., Maute, A., and Roble, R. G. (2009). Tropospheric tidal effects on the middle and upper atmosphere. Journal of Geophysical Research: Space Physics, 114(A1).

Hagan, M. E., Maute, A., Roble, R. G., Richmond, A. D., Immel, T. J., and England, S. L. (2007). Connections between deep tropical clouds and the Earth's ionosphere. Geophysical Research Letters, 34(20).

Hagan, M. E. and Roble, R. G. (2001). Modeling diurnal tidal variability with the National Center for Atmospheric Research thermosphere-ionosphere-mesosphereelectrodynamics general circulation model. Journal of Geophysical Research: Space Physics, 106(A11):24869-24882.

Hagan, M. E., Roble, R. G., and Hackney, J. (2001). Migrating thermospheric tides. Journal of Geophysical Research: Space Physics, 106(A7):12739-12752.

Hartman, W. A. and Heelis, R. A. (2007). Longitudinal variations in the equatorial vertical drift in the topside ionosphere. Journal of Geophysical Research: Space Physics, 112(A3).

Hartmann, D. L., Michelsen, M. L., and Klein, S. A. (1992). Seasonal Variations of Tropical Intraseasonal Oscillations: A 20-25-Day Oscillation in the Western Pacific. Journal of the Atmospheric Sciences, 49(14):1277-1289.

Hays, P. B., Wu, D. L., and HRDI Science Team, T. (1994). Observations of the Diurnal Tide from Space. Journal of the Atmospheric Sciences, 51(20):3077-3093.

Hermance, J. F. (2014). Interannual and Interseasonal Variations in Monthly Rainfall. In Historical Variability of Rainfall in the African East Sahel of Sudan, chapter 3, pages $43-57$. Springer.

Highfill, T., Georgi, P., and Dubria, D. (2019). Measuring the Value of the US Space Economy. Survey of Current Business, 99(12).

Hitchman, M. H. and Leovy, C. B. (1985). Diurnal Tide in the Equatorial Middle Atmosphere as Seen in LIMS Temperatures.

Hocke, K. and Kämpfer, N. (2009). Gap filling and noise reduction of unevenly sampled data by means of the Lomb-Scargle periodogram. Atmospheric Chemistry and Physics, 9(12):4197-4206.

Holton, J. R. (1975). The Dynamic Meteorology of the Stratosphere and Mesosphere. In Research supported by the National Science Foundation Boston, American Meteorological Society (Meteorological Monograph), volume 15, Boston, MA. American Meteorological Society.

Holton, J. R. (2004). An Introduction to Dynamic Meteorology. An Introduction to Dynamic Meteorology. Elsevier Science, 4th edition. 
Huang, F. T., Mayr, H. G., Reber, C. A., Russell, J. M., and Mengel, J. G. (2006). Stratospheric and mesospheric temperature variations for the quasi-biennial and semiannual (QBO and SAO) oscillations based on measurements from SABER (TIMED) and MLS (UARS). Annales Geophysicae, 24(8):2131-2149.

Huang, F. T. and Reber, C. A. (2003). Seasonal behavior of the semidiurnal and diurnal tides, and mean flows at $95 \mathrm{~km}$, based on measurements from the high resolution doppler imager (hrdi) on the upper atmosphere research satellite (uars). Journal of Geophysical Research: Atmospheres, 108(D12).

Huang, K. M., Liu, A. Z., Lu, X., Li, Z., Gan, Q., Gong, Y., Huang, C. M., Yi, F., and Zhang, S. D. (2013). Nonlinear coupling between quasi 2 day wave and tides based on meteor radar observations at Maui. Journal of Geophysical Research: Atmospheres, 118(19):10,936-10,943.

Huang, K. M., Yang, Z. X., Wang, R., Zhang, S. D., Huang, C. M., Yi, F., and Hu, F. (2018). A Statistical Study of Inertia Gravity Waves in the Lower Stratosphere Over the Arctic Region Based on Radiosonde Observations. Journal of Geophysical Research: Atmospheres, 123(10):4958-4976.

Iimura, H., Fritts, D. C., Wu, Q., Skinner, W. R., and Palo, S. E. (2010). Nonmigrating semidiurnal tide over the Arctic determined from TIMED Doppler Interferometer wind observations. Journal of Geophysical Research, 115(D6).

Immel, T. J., England, S. L., Mende, S. B., Heelis, R. A., Englert, C. R., Edelstein, J., Frey, H. U., Korpela, E. J., Taylor, E. R., Craig, W. W., Harris, S. E., Bester, M., Bust, G. S., Crowley, G., Forbes, J. M., Gérard, J.-C., Harlander, J. M., Huba, J. D., Hubert, B., Kamalabadi, F., Makela, J. J., Maute, A. I., Meier, R. R., Raftery, C., Rochus, P., Siegmund, O. H. W., Stephan, A. W., Swenson, G. R., Frey, S., Hysell, D. L., Saito, A., Rider, K. A., and Sirk, M. M. (2018). The Ionospheric Connection Explorer Mission: Mission Goals and Design. Space Science Reviews, 214(1):13.

Immel, T. J., Sagawa, E., England, S. L., Henderson, S. B., Hagan, M. E., Mende, S. B., Frey, H. U., Swenson, C. M., and Paxton, L. J. (2006). Control of equatorial ionospheric morphology by atmospheric tides. Geophysical Research Letters, 33(15).

Jacobi, C. (2012). 6 year mean prevailing winds and tides measured by VHF meteor radar over Collm $\left(51.3^{\circ} \mathrm{N}, 13.0^{\circ} \mathrm{E}\right)$. Journal of Atmospheric and Solar-Terrestrial Physics, 78-79:8-18.

Jacobi, C., Portnyagin, Y., Solovjova, T., Hoffmann, P., Singer, W., Fahrutdinova, A., Ishmuratov, R., Beard, A., Mitchell, N., Muller, H., Schminder, R., Kürschner, D., Manson, A., and Meek, C. (1999). Climatology of the semidiurnal tide at $52-56^{\circ} \mathrm{N}$ from ground-based radar wind measurements 1985-1995. Journal of Atmospheric and Solar-Terrestrial Physics, 61(13):975-991. 
Jin, H., Miyoshi, Y., Pancheva, D., Mukhtarov, P., Fujiwara, H., and Shinagawa, H. (2012). Response of migrating tides to the stratospheric sudden warming in 2009 and their effects on the ionosphere studied by a whole atmosphere-ionosphere model GAIA with COSMIC and TIMED/SABER observations. Journal of Geophysical Research: Space Physics, 117(A10).

Jones, M., Emmert, J. T., Drob, D. P., and Siskind, D. E. (2017). Middle atmosphere dynamical sources of the semiannual oscillation in the thermosphere and ionosphere. Geophysical Research Letters, 44(1):12-21.

Jones, M., Forbes, J. M., and Hagan, M. E. (2016). Solar cycle variability in mean thermospheric composition and temperature induced by atmospheric tides. Journal of Geophysical Research: Space Physics, 121(6):5837-5855.

Jones, M., Forbes, J. M., Hagan, M. E., and Maute, A. (2014). Impacts of vertically propagating tides on the mean state of the ionosphere-thermosphere system. Journal of Geophysical Research: Space Physics, 119(3):2197-2213.

Kamide, Y. and Chian, A., editors (2007). Thermosphere, pages 221-245. Springer Berlin Heidelberg, Berlin, Heidelberg.

Kato, S., Tsuda, T., and Watanabe, F. (1982). Thermal excitation of non-migrating tides. Journal of Atmospheric and Terrestrial Physics, 44(2):131-146.

Kazimirovsky, E., Herraiz, M., and De La Morena, B. A. (2003). Effects on the Ionosphere Due to Phenomena Occurring Below it. Surveys in Geophysics, 24(2):139184.

Kharin, V. V. and Scinocca, J. F. (2012). The impact of model fidelity on seasonal predictive skill. Geophysical Research Letters, 39(18):1-6.

Kil, H., Talaat, E. R., Oh, S.-J., Paxton, L. J., England, S. L., and Su, S.-Y. (2008). Wave structures of the plasma density and vertical $\mathrm{E} \times \mathrm{B}$ drift in low-latitude $\mathrm{F}$ region. Journal of Geophysical Research: Space Physics, 113(A9).

Kramer, H. J. (2002). Observation of the Earth and Its Environment: Survey of Missions and Sensors. Springer Berlin Heidelberg, Berlin, Heidelberg, 4 edition.

Kumar, K. K., Deepa, V., Antonita, T. M., and Ramkumar, G. (2008). Meteor radar observations of short-term tidal variabilities in the low-latitude mesospherelower thermosphere: Evidence for nonlinear wave-wave interactions. Journal of Geophysical Research, 113(D16).

Kumari, K. and Oberheide, J. (2020). QBO, ENSO, and Solar Cycle Effects in Short-Term Nonmigrating Tidal Variability on Planetary Wave Timescales From SABER-An Information-Theoretic Approach. Journal of Geophysical Research: Atmospheres, 125(6). 
Labitzke, K. and Loon, H. V. (1988). Associations between the 11-year solar cycle, the QBO and the atmosphere. Part I: the troposphere and stratosphere in the northern hemisphere in winter. Journal of Atmospheric and Terrestrial Physics, 50(3):197-206.

Laplace, P. S. (1799). Traité de mécanique céleste, volume 1. de l'Imprimerie de Crapelet.

Larson, J. W. (2012). Visualizing Climate Variability with Time-Dependent Probability Density Functions, Detecting It Using Information Theory. Procedia Computer Science, 9:917-926.

Leblanc, T., McDermid, I. S., and Ortland, D. A. (1999). Lidar observations of the middle atmospheric thermal tides and comparison with the High Resolution Doppler Imager and Global-Scale Wave Model: 1. Methodology and winter observations at Table Mountain $\left(34.4^{\circ} \mathrm{N}\right)$. Journal of Geophysical Research: Atmospheres, 104(D10):11917-11929.

Lei, J., Forbes, J. M., Liu, H.-L., Dou, X., Xue, X., Li, T., and Luan, X. (2011). Latitudinal variations of middle thermosphere: Observations and modeling. Journal of Geophysical Research: Space Physics, 116(A12).

Lieberman, R., Oberheide, J., Hagan, M., Remsberg, E., and Gordley, L. (2004). Variability of diurnal tides and planetary waves during November 1978-May 1979. Journal of Atmospheric and Solar-Terrestrial Physics, 66(6-9):517-528.

Lieberman, R. S. (1991). Nonmigrating Diurnal Tides in the Equatorial Middle Atmosphere. Journal of the Atmospheric Sciences, 48(8):1112-1123.

Lieberman, R. S. (1997). Long-term variations of zonal mean winds and $(1,1)$ driving in the equatorial lower thermosphere. Journal of Atmospheric and Solar-Terrestrial Physics, 59(13):1483-1490.

Lieberman, R. S. (1998). Intraseasonal variability of high-resolution Doppler imager winds in the equatorial mesosphere and lower thermosphere. Journal of Geophysical Research: Atmospheres, 103(D10):11221-11228.

Lieberman, R. S., Oberheide, J., and Talaat, E. R. (2013). Nonmigrating diurnal tides observed in global thermospheric winds. Journal of Geophysical Research: Space Physics, 118(11):7384-7397.

Lieberman, R. S., Ortland, D. A., Riggin, D. M., Wu, Q., and Jacobi, C. (2010). Momentum budget of the migrating diurnal tide in the mesosphere and lower thermosphere. Journal of Geophysical Research: Atmospheres, 115(D20).

Lieberman, R. S., Ortland, D. A., and Yarosh, E. S. (2003). Climatology and interannual variability of diurnal water vapor heating. Journal of Geophysical Research: Atmospheres, 108(D3). 
Lieberman, R. S., Riggin, D. M., Ortland, D. A., Nesbitt, S. W., and Vincent, R. A. (2007). Variability of mesospheric diurnal tides and tropospheric diurnal heating during 1997-1998. Journal of Geophysical Research Atmospheres, 112(20):1-17.

Lieberman, R. S., Riggin, D. M., Ortland, D. A., Oberheide, J., and Siskind, D. E. (2015). Global observations and modeling of nonmigrating diurnal tides generated by tide-planetary wave interactions. Journal of Geophysical Research: Atmospheres, 120(22):11,411-419,437.

Lin, C. H., Wang, W., Hagan, M. E., Hsiao, C. C., Immel, T. J., Hsu, M. L., Liu, J. Y., Paxton, L. J., Fang, T. W., and Liu, C. H. (2007). Plausible effect of atmospheric tides on the equatorial ionosphere observed by the FORMOSAT3/COSMIC: Three-dimensional electron density structures. Geophysical Research Letters, 34(11).

Lin, J. T., Lin, C. H., Chang, L. C., Huang, H. H., Liu, J. Y., Chen, A. B., Chen, C. H., and Liu, C. H. (2012). Observational evidence of ionospheric migrating tide modification during the 2009 stratospheric sudden warming. Geophysical Research Letters, 39(2).

Lindzen, R. S. (1966). ON THE THEORY OF THE DIURNAL TIDE. Monthly Weather Review, 94(5):295-301.

Lindzen, R. S. (1978). Effect of Daily Variations of Cumulonimbus Activity on the Atmospheric Semidiurnal Tide. Monthly Weather Review, 106(4):526-533.

Lindzen, R. S. and Hong, S.-s. (1974). Effects of Mean Winds and Horizontal Temperature Gradients on Solar and Lunar Semidiurnal Tides in the Atmosphere. Journal of the Atmospheric Sciences, 31(5):1421-1446.

Liu, H. (2016). Variability and predictability of the space environment as related to lower atmosphere forcing. Space Weather, 14(9):634-658.

Liu, H., Bardeen, C. G., Foster, B. T., Lauritzen, P., Liu, J., Lu, G., Marsh, D. R., Maute, A., McInerney, J. M., Pedatella, N. M., Qian, L., Richmond, A. D., Roble, R. G., Solomon, S. C., Vitt, F. M., and Wang, W. (2018). Development and Validation of the Whole Atmosphere Community Climate Model With Thermosphere and Ionosphere Extension (WACCM-X 2.0). Journal of Advances in Modeling Earth Systems, 10(2):381-402.

Liu, H., Sun, Y.-y., Miyoshi, Y., and Jin, H. (2017). ENSO effects on MLT diurnal tides: A 21 year reanalysis data-driven GAIA model simulation. Journal of Geophysical Research: Space Physics, 122(5):5539-5549.

Liu, H., Tsutsumi, M., and Liu, H. (2019). Vertical Structure of Terdiurnal Tides in the Antarctic MLT Region: 15-Year Observation Over Syowa $\left(69^{\circ} \mathrm{S}, 39^{\circ} \mathrm{E}\right)$. Geophysical Research Letters, 46(5):2364-2371. 
Liu, H.-L. (2014). WACCM-X Simulation of Tidal and Planetary Wave Variability in the Upper Atmosphere, chapter 16, pages 181-199. American Geophysical Union (AGU).

Liu, H.-L., Foster, B. T., Hagan, M. E., McInerney, J. M., Maute, A., Qian, L., Richmond, A. D., Roble, R. G., Solomon, S. C., Garcia, R. R., Kinnison, D., Marsh, D. R., Smith, A. K., Richter, J., Sassi, F., and Oberheide, J. (2010a). Thermosphere extension of the Whole Atmosphere Community Climate Model. Journal of Geophysical Research: Space Physics, 115(A12).

Liu, H. L., Li, T., She, C. Y., Oberheide, J., Wu, Q., Hagan, M. E., Xu, J., Roble, R. G., Mlynczak, M. G., and Russell, I. M. (2007). Comparative study of short-term diurnal tidal variability. Journal of Geophysical Research Atmospheres, 112(18):116.

Liu, H.-L., Wang, W., Richmond, A. D., and Roble, R. G. (2010b). Ionospheric variability due to planetary waves and tides for solar minimum conditions. Journal of Geophysical Research: Space Physics, 115(A6).

Lomb, N. R. (1976). Least-squares frequency analysis of unequally spaced data. Astrophysics and Space Science, 39(2):447-462.

Lu, X., Liu, A. Z., Oberheide, J., Wu, Q., Li, T., Li, Z., Swenson, G. R., and Franke, S. J. (2011). Seasonal variability of the diurnal tide in the mesosphere and lower thermosphere over Maui, Hawaii $\left(20.7^{\circ} \mathrm{N}, 156.3^{\circ} \mathrm{W}\right)$. Journal of Geophysical Research: Atmospheres, 116(D17).

Lu, X., Liu, H.-L., Liu, A. Z., Yue, J., McInerney, J. M., and Li, Z. (2012). Momentum budget of the migrating diurnal tide in the Whole Atmosphere Community Climate Model at vernal equinox. Journal of Geophysical Research: Atmospheres, 117(D7).

Luan, X., Dou, X., Lei, J., and Jiang, G. (2012). Terdiurnal migrating-tide signature in ionospheric total electron content. Journal of Geophysical Research: Space Physics, 117(A11).

Lühr, H., Rother, M., Häusler, K., Alken, P., and Maus, S. (2008). The influence of nonmigrating tides on the longitudinal variation of the equatorial electrojet. Journal of Geophysical Research: Space Physics, 113(A8).

Madden, R. A. and Julian, P. R. (1971). Detection of a 40-50 Day Oscillation in the Zonal Wind in the Tropical Pacific. Journal of the Atmospheric Sciences, 28(5):702-708.

Mayr, H. G. and Mengel, J. G. (2005). Interannual variations of the diurnal tide in the mesosphere generated by the quasi-biennial oscillation. Journal of Geophysical Research D: Atmospheres, 110(10):1-14. 
Mayr, H. G., Mengel, J. G., Talaat, E. R., Porter, H. S., and Chan, K. L. (2005a). Mesospheric non-migrating tides generated with planetary waves: I. Characteristics. Journal of Atmospheric and Solar-Terrestrial Physics, 67(11):959-980.

Mayr, H. G., Mengel, J. G., Talaat, E. R., Porter, H. S., and Chan, K. L. (2005b). Mesospheric non-migrating tides generated with planetary waves: II. Influence of gravity waves. Journal of Atmospheric and Solar-Terrestrial Physics, 67(11):981991.

McClintock, W. E., Eastes, R. W., Beland, S., Bryant, K. B., Burns, A. G., Correira, J., Danielll, R. E., Evans, J. S., Harper, C. S., Karan, D. K., Krywonos, A., Lumpe, J. D., Plummer, T. M., Solomon, S. C., Vanier, B. A., and Veibel, V. (2020). Global-Scale Observations of the Limb and Disk Mission Implementation: 2. Observations, Data Pipeline, and Level 1 Data Products. Journal of Geophysical Research: Space Physics, 125(5).

McCormack, J. P., Coy, L., and Hoppel, K. W. (2009). Evolution of the quasi 2day wave during January 2006. Journal of Geophysical Research: Atmospheres, 114(D20).

McLandress, C. (1997). Seasonal variability of the diurnal tide: Results from the Canadian middle atmosphere general circulation model. Journal of Geophysical Research: Atmospheres, 102(D25):29747-29764.

McLandress, C. (2002a). The Seasonal Variation of the Propagating Diurnal Tide in the Mesosphere and Lower Thermosphere. Part I: The Role of Gravity Waves and Planetary Waves. Journal of the Atmospheric Sciences, 59(5):893-906.

McLandress, C. (2002b). The Seasonal Variation of the Propagating Diurnal Tide in the Mesosphere and Lower Thermosphere. Part II: The Role of Tidal Heating and Zonal Mean Winds. Journal of the Atmospheric Sciences, 59(5):907-922.

McLandress, C., Plummer, D. A., and Shepherd, T. G. (2014). Technical Note: A simple procedure for removing temporal discontinuities in ERA-Interim upper stratospheric temperatures for use in nudged chemistry-climate model simulations. Atmospheric Chemistry and Physics, 14(3):1547-1555.

McLandress, C., Scinocca, J. F., Shepherd, T. G., Reader, M. C., and Manney, G. L. (2013). Dynamical Control of the Mesosphere by Orographic and Nonorographic Gravity Wave Drag during the Extended Northern Winters of 2006 and 2009. Journal of the Atmospheric Sciences, 70(7):2152-2169.

McLandress, C., Shepherd, G. G., and Solheim, B. H. (1996). Satellite observations of thermospheric tides: Results from the Wind Imaging Interferometer on UARS. Journal of Geophysical Research: Atmospheres, 101(D2):4093-4114.

McLandress, C. and Ward, W. (1994). Tidal/gravity wave interactions and their influence on the large-scale dynamics of the middle atmosphere: Model results. Journal of Geophysical Research: Atmospheres, 99(D4):8139-8155. 
McLandress, C., Ward, W. E., Fomichev, V. I., Semeniuk, K., Beagley, S. R., McFarlane, N. A., and Shepherd, T. G. (2006). Large-scale dynamics of the mesosphere and lower thermosphere: An analysis using the extended Canadian Middle Atmosphere Model. Journal of Geophysical Research Atmospheres, 111(17):1-16.

Mendillo, M., Rishbeth, H., Roble, R. G., and Wroten, J. (2002). Modelling F2layer seasonal trends and day-to-day variability driven by coupling with the lower atmosphere. Journal of Atmospheric and Solar-Terrestrial Physics, 64(18):19111931.

Meriwether, J., Faivre, M., Fesen, C., Sherwood, P., and Veliz, O. (2008). New results on equatorial thermospheric winds and the midnight temperature maximum. Annales Geophysicae, 26(3):447-466.

Meriwether, J. W., Makela, J. J., Huang, Y., Fisher, D. J., Buriti, R. A., Medeiros, A. F., and Takahashi, H. (2011). Climatology of the nighttime equatorial thermospheric winds and temperatures over Brazil near solar minimum. Journal of Geophysical Research: Space Physics, 116(A4).

Meriwether, J. W., Moody, J. W., Biondi, M. A., and Roble, R. G. (1986). Optical interferometric measurements of nighttime equatorial thermospheric winds at Arequipa, Peru. Journal of Geophysical Research, 91(A5):5557.

Millward, G. H., Müller-Wodarg, I. C. F., Aylward, A. D., Fuller-Rowell, T. J., Richmond, A. D., and Moffett, R. J. (2001). An investigation into the influence of tidal forcing on $\mathrm{F}$ region equatorial vertical ion drift using a global ionospherethermosphere model with coupled electrodynamics. Journal of Geophysical Research: Space Physics, 106(A11):24733-24744.

Mitchell, N. J., Pancheva, D., Middleton, H. R., and Hagan, M. E. (2002). Mean winds and tides in the Arctic mesosphere and lower thermosphere. Journal of Geophysical Research: Space Physics, 107(A1):1004.

Miyahara, S. and Forbes, J. M. (1991). Interactions between Gravity Waves and the Diurnal Tide in the Mesosphere and Lower Thermosphere. Journal of the Meteorological Society of Japan. Ser. II, 69(5):523-531.

Miyahara, S. and Miyoshi, Y. (1997). Migrating and non-migrating atmospheric tides simulated by a middle atmosphere general circulation model. Advances in Space Research, 20(6):1201-1207.

Miyahara, S., Miyoshi, Y., and Yamashita, K. (1999). Variations of migrating and non-migrating tides simulated by the Middle Atmosphere Circulation Model at Kyushu University. Advances in Space Research, 24(11):1549-1558.

Miyoshi, Y. (2006). Temporal variation of nonmigrating diurnal tide and its relation with the moist convective activity. Geophysical Research Letters, 33(11). 
Miyoshi, Y. and Fujiwara, H. (2003). Day-to-day variations of migrating diurnal tide simulated by a GCM from the ground surface to the exobase. Geophysical Research Letters, 30(15):1-4.

Miyoshi, Y. and Fujiwara, H. (2006). Day-to-day variations of migrating semidiurnal tide in the mesosphere and thermosphere. Memoirs of National Institute of Polar Research, Special Issue, (59):199-207.

Miyoshi, Y., Fujiwara, H., Forbes, J. M., and Bruinsma, S. L. (2009). Solar terminator wave and its relation to the atmospheric tide. Journal of Geophysical Research: Space Physics, 114(A7).

Moudden, Y. and Forbes, J. M. (2013). A decade-long climatology of terdiurnal tides using TIMED/SABER observations. Journal of Geophysical Research: Space Physics, 118(7):4534-4550.

Mukhtarov, P., Pancheva, D., and Andonov, B. (2009). Global structure and seasonal and interannual variability of the migrating diurnal tide seen in the SABER/TIMED temperatures between 20 and $120 \mathrm{~km}$. Journal of Geophysical Research: Space Physics, 114(2):1-17.

National Research Council (2013). Solar and Space Physics: A Science for a Technological Society. The National Academies Press, Washington, DC.

Nguyen, V. and Palo, S. (2013). Technique to produce daily estimates of the migrating diurnal tide using TIMED/SABER and EOS Aura/MLS. Journal of Atmospheric and Solar-Terrestrial Physics, 105-106:39-53.

Nischal, N., Oberheide, J., Mlynczak, M. G., Marsh, D. R., and Gan, Q. (2019). Solar Cycle Variability of Nonmigrating Tides in the 5.3 and $15 \mu \mathrm{m}$ Infrared Cooling of the Thermosphere (100-150 km) from SABER. Journal of Geophysical Research: Space Physics, 124(3):2338-2356.

Nozawa, S., Brekke, A., Maeda, S., Aso, T., Hall, C. M., Ogawa, Y., Buchert, S. C., Röttger, J., Richmond, A. D., Roble, R., and Fujii, R. (2005). Mean winds, tides, and quasi-2 day wave in the polar lower thermosphere observed in European Incoherent Scatter (EISCAT) 8 day run data in November 2003. Journal of Geophysical Research: Space Physics, 110(A12).

Oberheide, J., Forbes, J. M., Häusler, K., Wu, Q., and Bruinsma, S. L. (2009). Tropospheric tides from 80 to $400 \mathrm{~km}$ : Propagation, interannual variability, and solar cycle effects. Journal of Geophysical Research: Atmospheres, 114(D1).

Oberheide, J., Forbes, J. M., Zhang, X., and Bruinsma, S. L. (2011). Wave-driven variability in the ionosphere-thermosphere-mesosphere system from TIMED observations: What contributes to the "wave 4"? Journal of Geophysical Research: Space Physics, 116(A1). 
Oberheide, J., Hagan, M. E., and Roble, R. G. (2003). Tidal signatures and aliasing in temperature data from slowly precessing satellites. Journal of Geophysical Research: Space Physics, 108(A2):1-11.

Oberheide, J., Hagan, M. E., Roble, R. G., and Offermann, D. (2002). Sources of nonmigrating tides in the tropical middle atmosphere. Journal of Geophysical Research Atmospheres, 107(21):1-14.

Oberheide, J., Hagan, M. E., Ward, W. E., Riese, M., and Offermann, D. (2000). Modeling the diurnal tide for the Cryogenic Infrared Spectrometers and Telescopes for the Atmosphere (CRISTA) 1 time period. Journal of Geophysical Research, 105(A11):24917.

Oberheide, J., Shiokawa, K., Gurubaran, S., Ward, W. E., Fujiwara, H., Kosch, M. J., Makela, J. J., and Takahashi, H. (2015). The geospace response to variable inputs from the lower atmosphere: a review of the progress made by Task Group 4 of CAWSES-II. Progress in Earth and Planetary Science, 2(1):2.

Oberheide, J., Wu, Q., Killeen, T. L., Hagan, M. E., and Roble, R. G. (2006). Diurnal nonmigrating tides from TIMED Doppler Interferometer wind data: Monthly climatologies and seasonal variations. Journal of Geophysical Research, 111(A10).

Oliver, W. L., Martinis, C. R., Hickey, D. A., Wright, A. D., and Amory-Mazaudier, C. (2012). A nighttime temperature maximum in the thermosphere above Saint Santin in winter. Journal of Geophysical Research: Space Physics, 117(A6).

Ortland, D. A. (2005). A Study of the Global Structure of the Migrating Diurnal Tide Using Generalized Hough Modes. Journal of the Atmospheric Sciences, 62(8):26842702.

Ortland, D. A. (2017). Daily estimates of the migrating tide and zonal mean temperature in the mesosphere and lower thermosphere derived from SABER data. Journal of Geophysical Research: Atmospheres, 122(7):3754-3785.

Palo, S. E., Roble, R. G., and Hagan, M. E. (1999). Middle atmosphere effects of the quasi-two-day wave determined from a General Circulation Model. Earth, Planets and Space, 51(7-8):629-647.

Pancheva, D., Merzlyakov, E., Mitchell, N., Portnyagin, Y., Manson, A., Jacobi, C., Meek, C., Luo, Y., Clark, R., Hocking, W., MacDougall, J., Muller, H., Kürschner, D., Jones, G., Vincent, R., Reid, I., Singer, W., Igarashi, K., Fraser, G., Fahrutdinova, A., Stepanov, A., Poole, L., Malinga, S., Kashcheyev, B., and Oleynikov, A. (2002). Global-scale tidal variability during the PSMOS campaign of June-August 1999: interaction with planetary waves. Journal of Atmospheric and Solar-Terrestrial Physics, 64(17):1865-1896.

Pancheva, D., Mitchell, N., Middleton, H., and Muller, H. (2003). Variability of the semidiurnal tide due to fluctuations in solar activity and total ozone. Journal of Atmospheric and Solar-Terrestrial Physics, 65(1):1-19. 
Pancheva, D., Mukhtarov, P., and Andonov, B. (2009). Global structure, seasonal and interannual variability of the migrating semidiurnal tide seen in the SABER/TIMED temperatures (2002-2007). Annales Geophysicae, 27(2):687-703.

Pancheva, D., Mukhtarov, P., and Smith, A. K. (2013). Climatology of the migrating terdiurnal tide (TW3) in SABER/TIMED temperatures. Journal of Geophysical Research: Space Physics, 118(4):1755-1767.

Pancheva, D. V., Mukhtarov, P. J., Shepherd, M. G., Mitchell, N. J., Fritts, D. C., Riggin, D. M., Franke, S. J., Batista, P. P., Abdu, M. A., Batista, I. S., and Others (2006). Two-day wave coupling of the low-latitude atmosphere-ionosphere system. Journal of Geophysical Research: Space Physics, 111(A7).

Pedatella, N. M. and Forbes, J. M. (2010). Evidence for stratosphere sudden warmingionosphere coupling due to vertically propagating tides. Geophysical Research Letters, 37(11).

Pedatella, N. M. and Liu, H. (2013a). The influence of atmospheric tide and planetary wave variability during sudden stratosphere warmings on the low latitude ionosphere. Journal of Geophysical Research: Space Physics, 118(8):5333-5347.

Pedatella, N. M. and Liu, H.-L. (2012). Tidal variability in the mesosphere and lower thermosphere due to the El Niño-Southern Oscillation. Geophysical Research Letters, 39(19):n/a-n/a.

Pedatella, N. M. and Liu, H. L. (2013b). Influence of the El Niño Southern Oscillation on the middle and upper atmosphere. Journal of Geophysical Research: Space Physics, 118(5):2744-2755.

Pedatella, N. M., Liu, H.-L., and Hagan, M. E. (2012a). Day-to-day migrating and nonmigrating tidal variability due to the six-day planetary wave. Journal of Geophysical Research: Space Physics, 117(A6).

Pedatella, N. M., Liu, H.-L., Richmond, A. D., Maute, A., and Fang, T.-W. (2012b). Simulations of solar and lunar tidal variability in the mesosphere and lower thermosphere during sudden stratosphere warmings and their influence on the low-latitude ionosphere. Journal of Geophysical Research: Space Physics, 117(A8).

Pedatella, N. M., Oberheide, J., Sutton, E. K., Liu, H., Anderson, J. L., and Raeder, K. (2016). Short-term nonmigrating tide variability in the mesosphere, thermosphere, and ionosphere. Journal of Geophysical Research: Space Physics, 121(4):3621-3633.

Reber, C. A. (1993). The upper atmosphere research satellite (UARS). Geophysical Research Letters, 20(12):1215-1218.

Reddi, C. R., Rajeev, K., and Geetha, R. (1993). Tidal winds in the radio-meteor region over Trivandrum $\left(8.5^{\circ} \mathrm{N}, 77^{\circ} \mathrm{E}\right)$. Journal of Atmospheric and Terrestrial Physics, 55(9):1219-1231. 
Remsberg, E. E., Marshall, B. T., Garcia-Comas, M., Krueger, D., Lingenfelser, G. S., Martin-Torres, J., Mlynczak, M. G., Russell, J. M., Smith, A. K., Zhao, Y., Brown, C., Gordley, L. L., Lopez-Gonzalez, M. J., Lopez-Puertas, M., She, C. Y., Taylor, M. J., and Thompson, R. E. (2008). Assessment of the quality of the version 1.07 temperature-versus-pressure profiles of the middle atmosphere from TIMED/SABER. Journal of Geophysical Research Atmospheres, 113(17):1-27.

Riggin, D. M. and Lieberman, R. S. (2013). Variability of the diurnal tide in the equatorial MLT. Journal of Atmospheric and Solar-Terrestrial Physics, 102:198206.

Rishbeth, H. and Mendillo, M. (2001). Patterns of F2-layer variability. Journal of Atmospheric and Solar-Terrestrial Physics, 63:1661-1680.

Roble, R. (2003). THERMOSPHERE. In Holton, J. R., editor, Encyclopedia of Atmospheric Sciences, pages 2282-2290. Elsevier, Oxford.

Roble, R. G. and Ridley, E. C. (1994). A thermosphere-ionosphere-mesosphereelectrodynamics general circulation model (time-GCM): Equinox solar cycle minimum simulations (30-500 km). Geophysical Research Letters, 21(6):417-420.

Ross, W. (2020). Remarks by Secretary of Commerce Wilbur Ross at A New Space Race: Getting to the Trillion-Dollar Space Economy World Economic Forum, Davos, Switzerland.

Ruan, H., Lei, J., Dou, X., Wan, W., and Liu, Y. C.-M. (2014). Midnight density maximum in the thermosphere from the CHAMP observations. Journal of Geophysical Research: Space Physics, 119(5):3741-3746.

Russell, J. M., Mlynczak, M. G., Gordley, L. L., Tansock Jr., J. J., and Esplin, R. W. (1999). Overview of the SABER experiment and preliminary calibration results. Proc. SPIE, 3756(October):277-288.

Sagawa, E., Immel, T. J., Frey, H. U., and Mende, S. B. (2005). Longitudinal structure of the equatorial anomaly in the nighttime ionosphere observed by IMAGE/FUV. Journal of Geophysical Research, 110(A11):A11302.

Sakazaki, T., Fujiwara, M., and Zhang, X. (2013). Interpretation of the vertical structure and seasonal variation of the diurnal migrating tide from the troposphere to the lower mesosphere. Journal of Atmospheric and Solar-Terrestrial Physics, 105-106:66-80.

Sakazaki, T., Fujiwara, M., Zhang, X., Hagan, M. E., and Forbes, J. M. (2012). Diurnal tides from the troposphere to the lower mesosphere as deduced from TIMED/SABER satellite data and six global reanalysis data sets. Journal of Geophysical Research Atmospheres, 117(13):1-21. 
Sassi, F., McCormack, J. P., and McDonald, S. E. (2019). Whole Atmosphere Coupling on Intraseasonal and Interseasonal Time Scales: A Potential Source of Increased Predictive Capability. Radio Science, 54(11):913-933.

Scargle, J. D. (1982). Studies in astronomical time series analysis. II - Statistical aspects of spectral analysis of unevenly spaced data. The Astrophysical Journal, 263:835.

Scherliess, L., Thompson, D. C., and Schunk, R. W. (2008). Longitudinal variability of low-latitude total electron content: Tidal influences. Journal of Geophysical Research: Space Physics, 113(1).

Scinocca, J. F., McFarlane, N. A., Lazare, M., Li, J., and Plummer, D. (2008). Technical Note: The CCCma third generation AGCM and its extension into the middle atmosphere. Atmospheric Chemistry and Physics, 8(23):7055-7074.

Semeniuk, K., Fomichev, V. I., McConnell, J. C., Fu, C., Melo, S. M. L., and Usoskin, I. G. (2011). Middle atmosphere response to the solar cycle in irradiance and ionizing particle precipitation. Atmospheric Chemistry and Physics, 11(10):50455077 .

She, C. Y., Li, T., Collins, R. L., Yuan, T., Williams, B. P., Kawahara, T. D., Vance, J. D., Acott, P., Krueger, D. A., Liu, H.-L., and Hagan, M. E. (2004). Tidal perturbations and variability in the mesopause region over Fort Collins, CO (41N, 105W): Continuous multi-day temperature and wind lidar observations. Geophysical Research Letters, 31(24).

Shepherd, M. G., Beagley, S. R., and Fomichev, V. I. (2014). Stratospheric warming influence on the mesosphere/lower thermosphere as seen by the extended CMAM. Ann. Geophys., 32(6):589-608.

Siebert, M. (1961). Atmospheric Tides. volume 7 of Advances in Geophysics, pages 105-187. Elsevier.

Singh, D. and Gurubaran, S. (2017). Variability of diurnal tide in the MLT region over Tirunelveli $\left(8.7^{\circ} \mathrm{N}\right)$, India: Consistency between ground- and space-based observations. Journal of Geophysical Research: Atmospheres, 122(5):2696-2713.

Siskind, D. E., Drob, D. P., Dymond, K. F., and McCormack, J. P. (2014). Simulations of the effects of vertical transport on the thermosphere and ionosphere using two coupled models. Journal of Geophysical Research: Space Physics, 119(2):11721185 .

Smith, A. K. (2012). Global Dynamics of the MLT. Surveys in Geophysics, 33(6):1177-1230.

Smith, A. K., Harvey, V. L., Mlynczak, M. G., Funke, B., García-Comas, M., Hervig, M., Kaufmann, M., Kyrölä, E., López-Puertas, M., McDade, I., Randall, C. E., Russell III, J. M., Sheese, P. E., Shiotani, M., Skinner, W. R., Suzuki, M., and 
Walker, K. A. (2013). Satellite observations of ozone in the upper mesosphere. Journal of Geophysical Research: Atmospheres, 118(11):5803-5821.

Smith, A. K. and Ortland, D. A. (2001). Modeling and Analysis of the Structure and Generation of the Terdiurnal Tide. Journal of the Atmospheric Sciences, 58(21):3116-3134.

Sridharan, S. (2019). Seasonal Variations of Low-Latitude Migrating and Nonmigrating Diurnal and Semidiurnal Tides in TIMED-SABER Temperature and Their Relationship With Source Variations. Journal of Geophysical Research: Space Physics, 124(5):3558-3572.

Sun, Y.-Y., Liu, H., Miyoshi, Y., Liu, L., and Chang, L. C. (2018). El Niño-Southern Oscillation effect on quasi-biennial oscillations of temperature diurnal tides in the mesosphere and lower thermosphere. Earth, Planets and Space, 70(1):85.

Svoboda, A. A., Forbes, J. M., and Miyahara, S. (2005). A space-based climatology of diurnal MLT tidal winds, temperatures and densities from UARS wind measurements. Journal of Atmospheric and Solar-Terrestrial Physics, 67(16):1533-1543.

Talaat, E. R. and Lieberman, R. S. (1999). Nonmigrating Diurnal Tides in Mesospheric and Lower-Thermospheric Winds and Temperatures. Journal of the Atmospheric Sciences, 56(24):4073-4087.

Tapping, K. F. (2013). The $10.7 \mathrm{~cm}$ solar radio flux ( F 10.7 ). Space Weather, 11(7):394-406.

Taylor, G. I. (1936). The oscillations of the atmosphere. Proceedings of the Royal Society of London. Series A-Mathematical and Physical Sciences, 156(888):318326.

Teitelbaum, H. and Vial, F. (1991). On tidal variability induced by nonlinear interaction with planetary waves. Journal of Geophysical Research: Space Physics, 96(A8):14169-14178.

Teitelbaum, H., Vial, F., Manson, A., Giraldez, R., and Massebeuf, M. (1989). Nonlinear interaction between the diurnal and semidiurnal tides: terdiurnal and diurnal secondary waves. Journal of Atmospheric and Terrestrial Physics, 51(7-8):627-634.

Thayaparan, T. (1997). The terdiurnal tide in the mesosphere and lower thermosphere over London, Canada $\left(43^{\circ} \mathrm{N}, 81^{\circ} \mathrm{W}\right)$. Journal of Geophysical Research: Atmospheres, 102(D18):21695-21708.

Torrence, C. and Compo, G. P. (1998). A Practical Guide to Wavelet Analysis. Bulletin of the American Meteorological Society, 79(1):61-78.

Tsuda, T. and Kato, S. (1989). Diurnal Non-Migrating Tides Excited by a Differential Heating Due to Land-Sea Distribution. Journal of the Meteorological Society of Japan. Ser. II, 67(1):43-55. 
Tsuda, T., Kato, S., Manson, A. H., and Meek, C. E. (1988). Characteristics of semidiurnal tides observed by the Kyoto meteor radar and Saskatoon mediumfrequency radar. Journal of Geophysical Research: Atmospheres, 93(D6):70277036 .

Vergados, P., Liu, G., Mannucci, A. J., and Janches, D. (2018). Equatorial Intraseasonal Temperature Oscillations in the Lower Thermosphere From SABER. Geophysical Research Letters, 45(20):10,810-893,902.

Vial, F., Lott, F., and Teitelbaum, H. (1994). A possible signal of the El NiñoSouthern Oscillation in time series of the diurnal tide. Geophysical Research Letters, 21(15):1603-1606.

Vincent, R., Tsuda, T., and Kato, S. (1989). Asymmetries in mesospheric tidal structure. Journal of Atmospheric and Terrestrial Physics, 51(7-8):609-616.

Vincent, R. A. (2015). The dynamics of the mesosphere and lower thermosphere: a brief review. Progress in Earth and Planetary Science, 2(1):4.

Vincent, R. A., Kovalam, S., Fritts, D. C., and Isler, J. R. (1998). Long-term MF radar observations of solar tides in the low-latitude mesosphere: Interannual variability and comparisons with the GSWM. Journal of Geophysical Research: Atmospheres, 103(D8):8667-8683.

Vitharana, A., Zhu, X., Du, J., Oberheide, J., and Ward, W. E. (2019). Statistical modeling of tidal weather in the mesosphere and lower thermosphere. Journal of Geophysical Research: Atmospheres, 124(16):9011-9027.

von Zahn, U., Höffner, J., Eska, V., and Alpers, M. (1996). The mesopause altitude: Only two distinctive levels worldwide? Geophysical Research Letters, 23(22):32313234.

Wallace, J. M. and Hartranft, F. R. (1969). DIURNAL WIND VARIATIONS, SURFACE TO 30 KILOMETERS. Monthly Weather Review, 97(6):446-455.

Wallace, J. M. and Tadd, R. F. (1974). Some Further Results Concerning the Vertical Structure of Atmospheric Tidal Motions Within the Lowest 30 Kilometers. Monthly Weather Review, 102(11):795-803.

Walterscheid, R. L. and Venkateswaran, S. V. (1979). Influence of Mean Zonal Motion and Meridional Temperature Gradients on the Solar Semidiurnal Atmospheric Tide: A Spectral Study. Part II: Numerical Results. Journal of the Atmospheric Sciences, 36(9):1636-1662.

Wan, W., Liu, L., Pi, X., Zhang, M.-L., Ning, B., Xiong, J., and Ding, F. (2008). Wavenumber-4 patterns of the total electron content over the low latitude ionosphere. Geophysical Research Letters, 35(12). 
Ward, W. E., Fomichev, V. I., and Beagley, S. (2005). Nonmigrating tides in equinox temperature fields from the Extended Canadian Middle Atmosphere Model (CMAM). Geophysical Research Letters, 32(3).

Ward, W. E., Oberheide, J., Goncharenko, L. P., Nakamura, T., Hoffmann, P., Singer, W., Chang, L. C., Du, J., Wang, D.-Y., Batista, P., Clemesha, B., Manson, a. H., Riggin, D. M., She, C.-Y., Tsuda, T., and Yuan, T. (2010). On the consistency of model, ground-based, and satellite observations of tidal signatures: Initial results from the CAWSES tidal campaigns. Journal of Geophysical Research, 115(D7):D07107.

Ward, W. E., Oberheide, J., Riese, M., Preusse, P., and Offermann, D. (1999). Tidal signatures in temperature data from CRISTA 1 mission. Journal of Geophysical Research: Atmospheres, 104(D13):16391-16403.

Warner, K. and Oberheide, J. (2014). Nonmigrating tidal heating and MLT tidal wind variability due to the El Niño-Southern Oscillation. Journal of Geophysical Research: Atmospheres, 119(3):1249-1265.

Williams, C. R. and Avery, S. K. (1996). Diurnal nonmigrating tidal oscillations forced by deep convective clouds. Journal of Geophysical Research: Atmospheres, 101(D2):4079-4091.

Wolter, K. and Timlin, M. S. (1998). Measuring the strength of ENSO events: How does 1997/98 rank? Weather, 53(9):315-324.

Wolter, K. and Timlin, M. S. (2011). El Niño/Southern Oscillation behaviour since 1871 as diagnosed in an extended multivariate ENSO index (MEI.ext). International Journal of Climatology, 31(7):1074-1087.

Wu, Q., Ortland, D. A., Killeen, T. L., Roble, R. G., Hagan, M. E., Liu, H.-L., Solomon, S. C., Xu, J., Skinner, W. R., and Niciejewski, R. J. (2008). Global distribution and interannual variations of mesospheric and lower thermospheric neutral wind diurnal tide: 1. Migrating tide. Journal of Geophysical Research: Space Physics, 113(A5).

Xia, Y., Fabian, P., Stohl, A., and Winterhalter, M. (1999). Forest climatology: Estimation of missing values for Bavaria, Germany. Agricultural and Forest Meteorology, 96(1-3):131-144.

Xu, J., Liu, H.-L., Yuan, W., Smith, A. K., Roble, R. G., Mertens, C. J., Russell III, J. M., and Mlynczak, M. G. (2007). Mesopause structure from Thermosphere, Ionosphere, Mesosphere, Energetics, and Dynamics (TIMED)/Sounding of the Atmosphere Using Broadband Emission Radiometry (SABER) observations. Journal of Geophysical Research: Atmospheres, 112(D9).

Xu, J., Smith, A. K., Jiang, G., and Yuan, W. (2010). Seasonal variation of the Hough modes of the diurnal component of ozone heating evaluated from Aura 
Microwave Limb Sounder observations. Journal of Geophysical Research: Atmospheres, 115(D10).

Xu, J., Smith, A. K., Liu, H.-L., Yuan, W., Wu, Q., Jiang, G., Mlynczak, M. G., Russell, J. M., and Franke, S. J. (2009). Seasonal and quasi-biennial variations in the migrating diurnal tide observed by Thermosphere, Ionosphere, Mesosphere, Energetics and Dynamics (TIMED). Journal of Geophysical Research, 114(D13):D13107.

Yamashita, C., Liu, H.-L., and Chu, X. (2010). Responses of mesosphere and lower thermosphere temperatures to gravity wave forcing during stratospheric sudden warming. Geophysical Research Letters, 37(9).

Yamashita, K., Miyahara, S., Miyoshi, Y., Kawano, K., and Ninomiya, J. (2002). Seasonal variation of non-migrating semidiurnal tide in the polar MLT region in a general circulation model. Journal of Atmospheric and Solar-Terrestrial Physics, 64(8-11):1083-1094.

Yamazaki, Y., Liu, H., Sun, Y., Miyoshi, Y., Kosch, M. J., and Mlynczak, M. G. (2017). Quasi-biennial oscillation of the ionospheric wind dynamo. Journal of Geophysical Research: Space Physics, 122(3):3553-3569.

Yamazaki, Y. and Richmond, A. D. (2013). A theory of ionospheric response to upward-propagating tides: Electrodynamic effects and tidal mixing effects. Journal of Geophysical Research: Space Physics, 118(9):5891-5905.

Yang, C., Smith, A. K., Li, T., and Dou, X. (2018). The Effect of the MaddenJulian Oscillation on the Mesospheric Migrating Diurnal Tide: A Study Using SD-WACCM. Geophysical Research Letters, 45(10):5105-5114.

Yeh, W.-H., Liu, J.-Y., Huang, C.-Y., and Chen, S.-P. (2014). Explanation of the sporadic- E layer formation by comparing FORMOSAT-3/COSMIC data with meteor and wind shear information. Journal of Geophysical Research: Atmospheres, 119(8):4568-4579.

Yi, F. (2001). Short-term variability and temporary structures of tides and mean wind in the polar summer mesosphere. Journal of Atmospheric and Solar-Terrestrial Physics, 63(8):749-757.

Younger, P. T., Pancheva, D., Middleton, H. R., and Mitchell, N. J. (2002). The 8hour tide in the Arctic mesosphere and lower thermosphere. Journal of Geophysical Research: Space Physics, 107(A12):SIA 2-1-SIA 2-11.

Yu, Y., Wan, W., Ning, B., Liu, L., Wang, Z., Hu, L., and Ren, Z. (2013). Tidal wind mapping from observations of a meteor radar chain in December 2011. Journal of Geophysical Research: Space Physics, 118(5):2321-2332. 
Zeng, Z., Randel, W., Sokolovskiy, S., Deser, C., Kuo, Y.-H., Hagan, M., Du, J., and Ward, W. (2008). Detection of migrating diurnal tide in the tropical upper troposphere and lower stratosphere using the Challenging Minisatellite Payload radio occultation data. Journal of Geophysical Research, 113(D3).

Zhang, X., Forbes, J. M., and Hagan, M. E. (2012). Seasonal-latitudinal variation of the eastward-propagating diurnal tide with zonal wavenumber 3 in the MLT: Influences of heating and background wind distribution. Journal of Atmospheric and Solar-Terrestrial Physics, 78-79:37-43.

Zhang, X., Forbes, J. M., Hagan, M. E., Russell, J. M., Palo, S. E., Mertens, C. J., and Mlynczak, M. G. (2006). Monthly tidal temperatures 20-120 km from TIMED/SABER. Journal of Geophysical Research, 111(A10).

Zhou, Y.-L., Wang, L., Xiong, C., Lühr, H., and Ma, S.-Y. (2016). The solar activity dependence of nonmigrating tides in electron density at low and middle latitudes observed by CHAMP and GRACE. Annales Geophysicae, 34(4):463-472.

Zhu, X., Yee, J. H., Talaat, E. R., Mlynczak, M., Gordley, L., Mertens, C., and Russell, I. M. (2005). An algorithm for extracting zonal mean and migrating tidal fields in the middle atmosphere from satellite measurements: Applications to TIMED/SABER-measured temperature and tidal modeling. Journal of Geophysical Research D: Atmospheres, 110(2):1-14. 


\section{Appendix A: Acronyms}

\begin{tabular}{|c|c|}
\hline AIMI & Atmosphere-Ionosphere-Magnetosphere Interactions \\
\hline CAWSES & Climate and Weather of the Sun-Earth System \\
\hline CCCma & Canadian Centre for Climate Modeling and Analysis \\
\hline CCM & Climate Community Model \\
\hline CHAMP & Challenging Minisatellite Payload \\
\hline CME & Coronal Mass Ejections \\
\hline COSMIC & $\begin{array}{l}\text { Constellation Observing System for Meteorology, Iono- } \\
\text { sphere and Climate }\end{array}$ \\
\hline CRISTA & $\begin{array}{l}\text { Cryogenic Infrared Spectrometers and Telescopes for the } \\
\text { Atmosphere }\end{array}$ \\
\hline CSA & Canadian Space Agency \\
\hline DART & Data Assimilation Research Testbed \\
\hline DYNAMIC & Dynamical Neutral Atmosphere-Ionosphere Coupling \\
\hline eCMAM & extended Canadian Middle Atmospheric Model \\
\hline ECMWF & European Center for Medium-Range Weather Forecasts \\
\hline EEJ & Equatorial Electrojet \\
\hline EIA & Equatorial Ionization Anomaly \\
\hline ENSO & El Niño-Southern Oscillation \\
\hline ERA & ECMWF Re-Analysis \\
\hline EUV & Extreme Ultra-Violet \\
\hline FUV & Far Ultraviolet Imager \\
\hline GAIA & $\begin{array}{l}\text { Ground-to-topside model of Atmosphere and Ionosphere for } \\
\text { Aeronomy }\end{array}$ \\
\hline GCM & General Circulation Models \\
\hline GDC & Geospace Dynamics Constellation \\
\hline GIP & Global Ionosphere-Plasmasphere \\
\hline GOLD & Global-scale Observations of the Limb and Disk \\
\hline GPS & Global Positioning System \\
\hline GRACE & Gravity Recovery and Climate Experiment \\
\hline GSWM & Global-Scale Wave Model \\
\hline GW & Gravity Wave \\
\hline GWD & Gravity Wave Drag \\
\hline HadISST & Hadley Center's Sea ice and Sea surface Temperature \\
\hline $\mathrm{HF}$ & High Frequency \\
\hline HRDI & High-Resolution Doppler Imager \\
\hline
\end{tabular}




$\begin{array}{ll}\text { ICON } & \text { Ionospheric Connection Explorer } \\ \text { IEC } & \text { Integrated Electron Content } \\ \text { IMAGE } & \text { Imager for Magnetopause-to-Aurora Global Exploration } \\ \text { IRI } & \text { International Reference Ionospher } \\ \text { ISS } & \text { International Space Station } \\ \text { IT } & \text { Ionosphere-Thermosphere } \\ \text { ITM } & \text { Ionosphere-Thermosphere-Mesosphere } \\ \text { LIDAR } & \text { Light Detection and Ranging } \\ \text { LIMS } & \text { Limb Infrared Monitor of the Stratosphere } \\ \text { LTE } & \text { Local Thermal Equilibrium } \\ \text { MERRA } & \text { Modern Era Retrospective analysis for Research and Appli- } \\ & \text { cations } \\ \text { MF } & \text { Medium Frequency } \\ \text { MJO } & \text { Madden-Julian Oscillation } \\ \text { MLS } & \text { Microwave Limb Sounder } \\ \text { MLT } & \text { Mesosphere and Lower Thermosphere } \\ \text { MR } & \text { Meteor Radar } \\ \text { MTM } & \text { Midnight Temperature Maximum } \\ \text { Q10DW } & \text { Quasi-10-Day Wave } \\ \text { QBO } & \text { Quasi-Biennial Oscillation } \\ \text { QTDW } & \text { quasi two-day wav } \\ \text { RADAR } & \text { Radio Wave Detection and Ranging } \\ \text { RV } & \text { Relative Variance } \\ \text { SABER } & \text { Sounding of the Atmosphere using Broadband Emission Ra- } \\ & \text { diometry } \\ \text { SPW } & \text { Stationary Planetary Waves } \\ \text { SSI } & \text { Spectral Solar Irradiance } \\ \text { SST } & \text { Sea Surface Temperature } \\ \text { SSW } & \text { Sudden Stratospheric Warming } \\ \text { TEC } & \text { Total Electron Content } \\ \text { TIDI } & \text { TIMED Doppler Interferometer } \\ \text { TIE-GCM } & \text { Thermosphere-Ionosphere- Electrodynamics General Circu- } \\ & \text { lation Model } \\ \text { TIMED } & \text { Thermosphere Ionosphere Mesosphere Energetics and Dy- } \\ & \text { namics } \\ \text { TIME-GCM } & \text { Thermosphere Ionosphere Mesosphere Electrodynamics } \\ & \text { General Circulation Model } \\ \text { UARS } & \text { Upper Atmosphere Research Satellite } \\ \text { WACCM-X } & \text { Whole Atmosphere Community Climate Model with ther- } \\ \text { WAM } & \text { mosphere and ionosphere extension } \\ \text { WINDII } & \text { Whole Atmosphere Model } \\ & \text { Wind Imaging Interferometer } \\ & \end{array}$




\section{Appendix B: Comparision of DW1 T Between SABER Version 1.07 and 2.0}
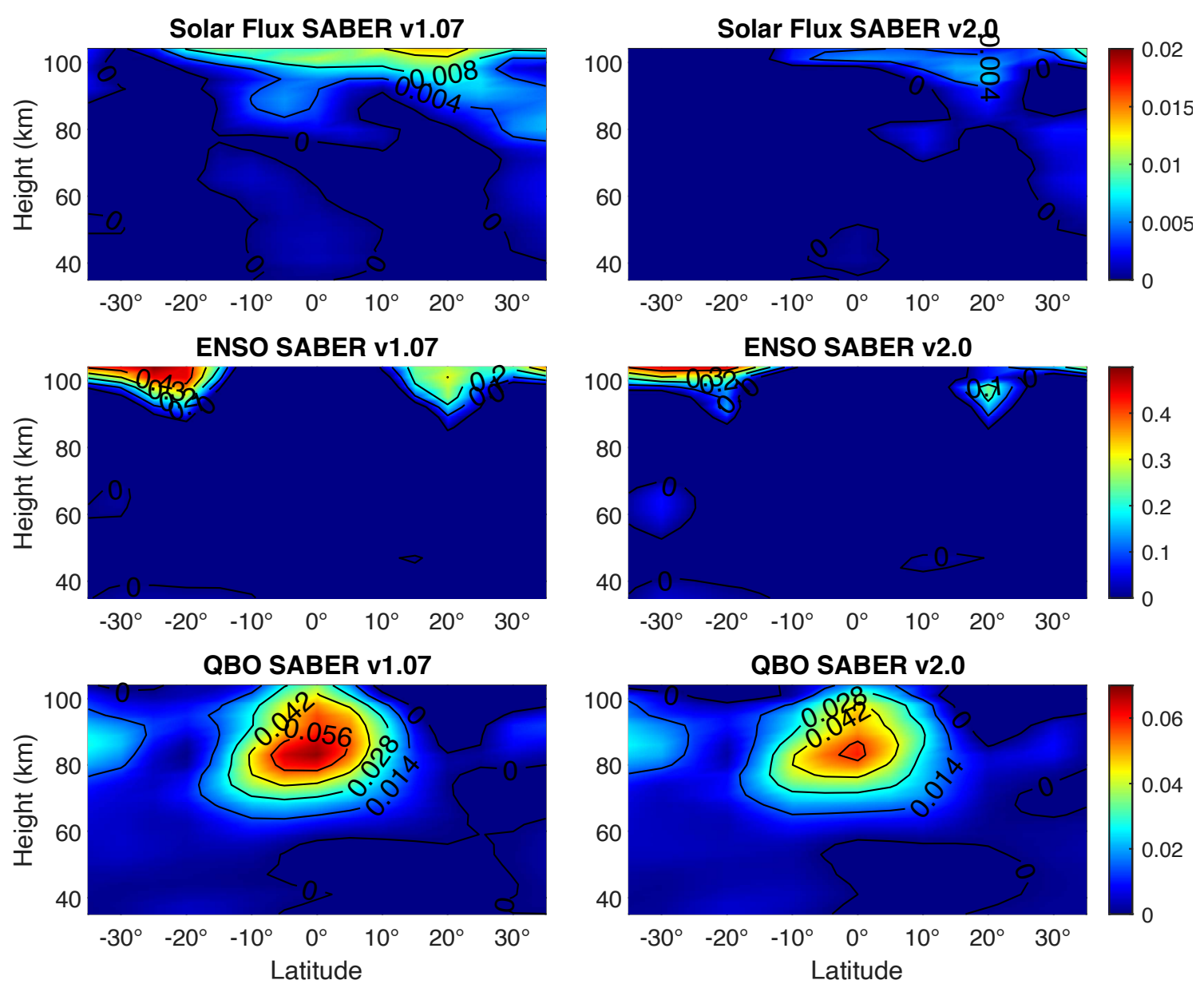

Figure B1. Coefficients of the DW1 T variability due to deterministic interannual variability indices, e.g., solar cycle (top panel), ENSO (middle panel), and QBO (bottom panel), in the SABER version 1.07 (left) and SABER version 2.0 (right) as a function of height and latitude. 

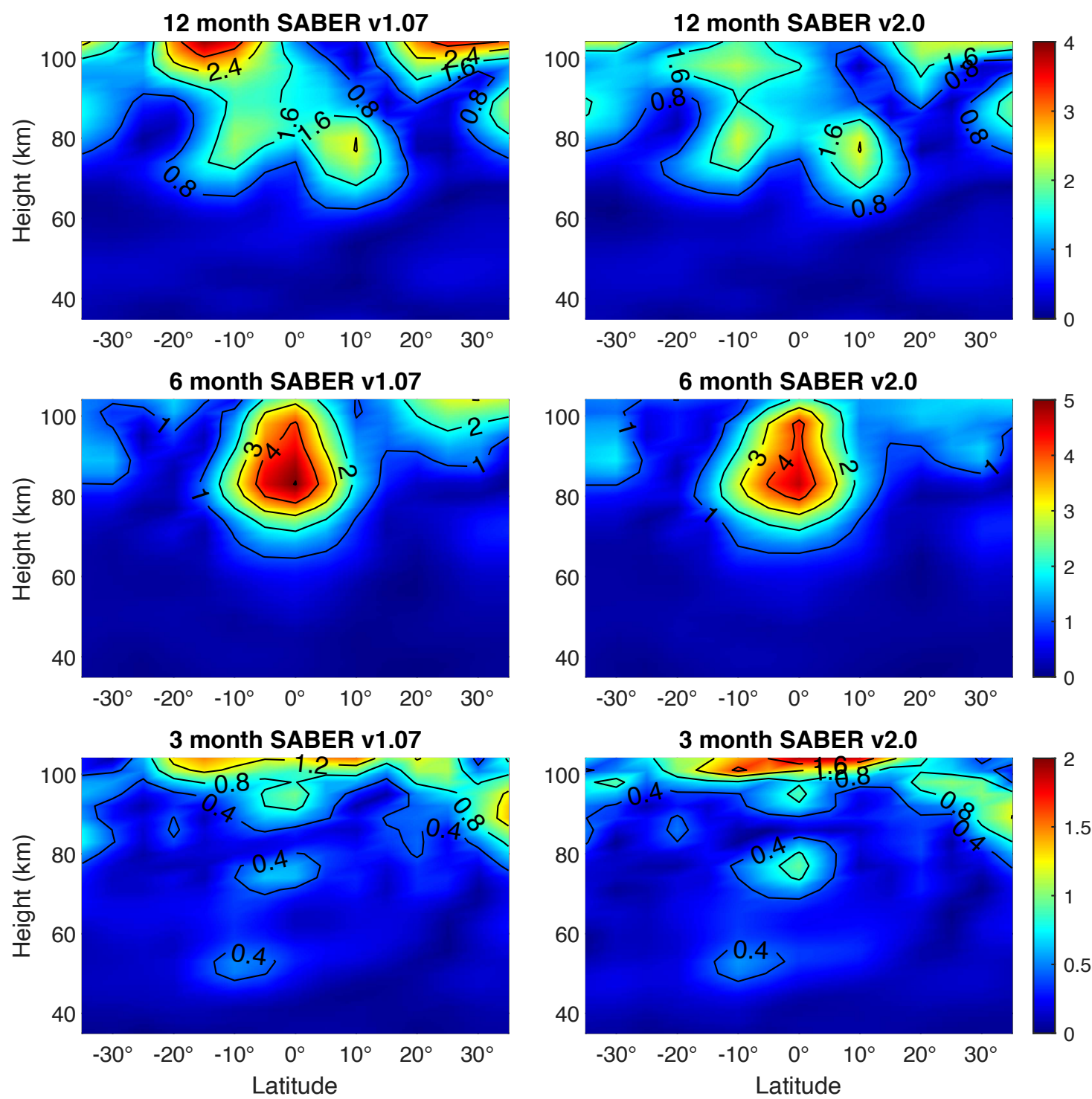

Figure B2. Amplitude of the DW1 T variability as a function of height and latitude due to the seasonal harmonics: 12, 6, and 3 months in the SABER version 1.07 (left) and SABER version 2.0 (right). 
Appendix C: Correlations of Linear, Nonlinear Advection, Solar Heating, and Heating Rate
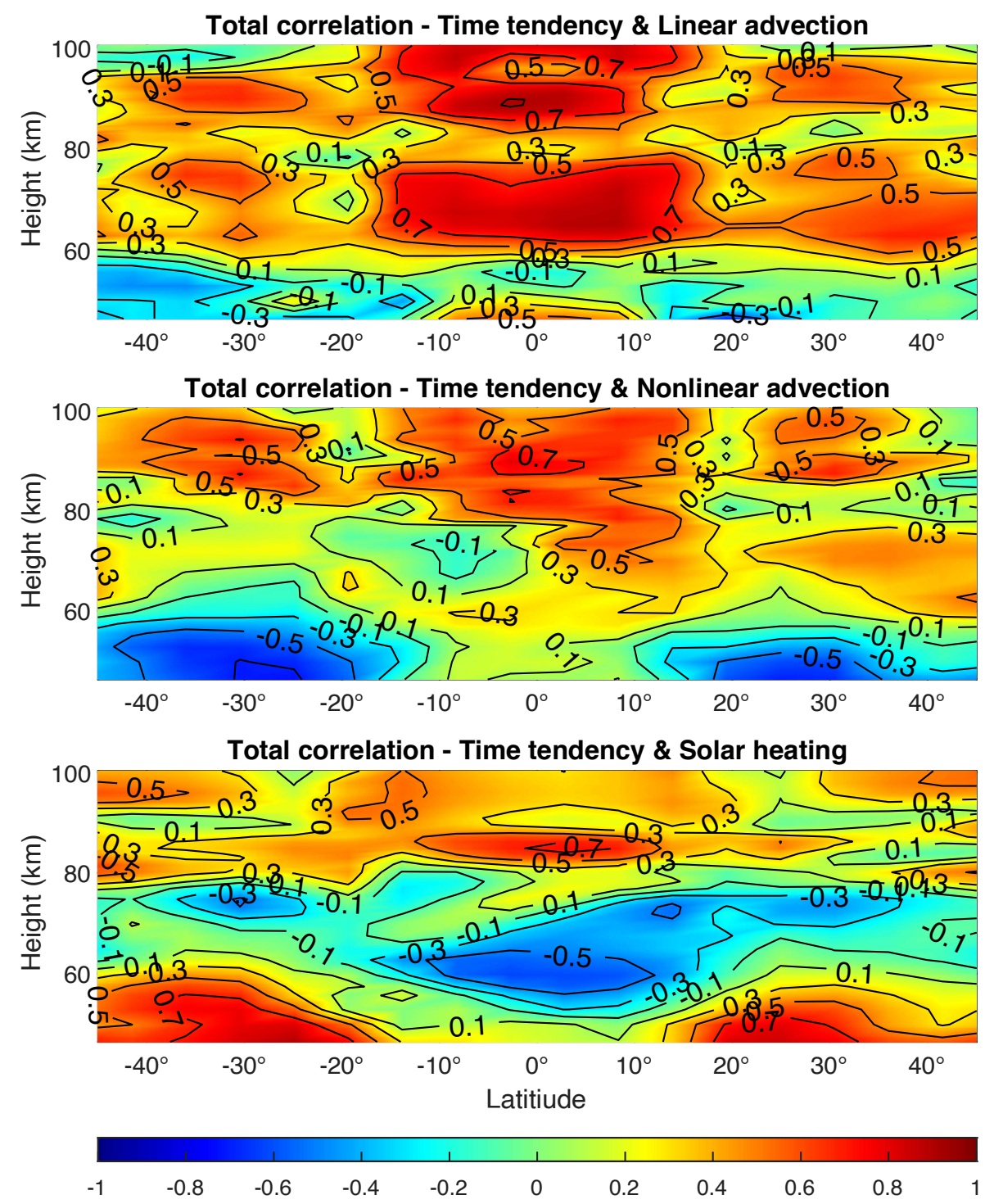

Figure C1. Correlation between DW1 T time tendency and linear advection (top panel), nonlinear advection (middle panel), and solar heating (bottom panel) in the mesosphere as a function of latitude and height from the eCMAM30. 


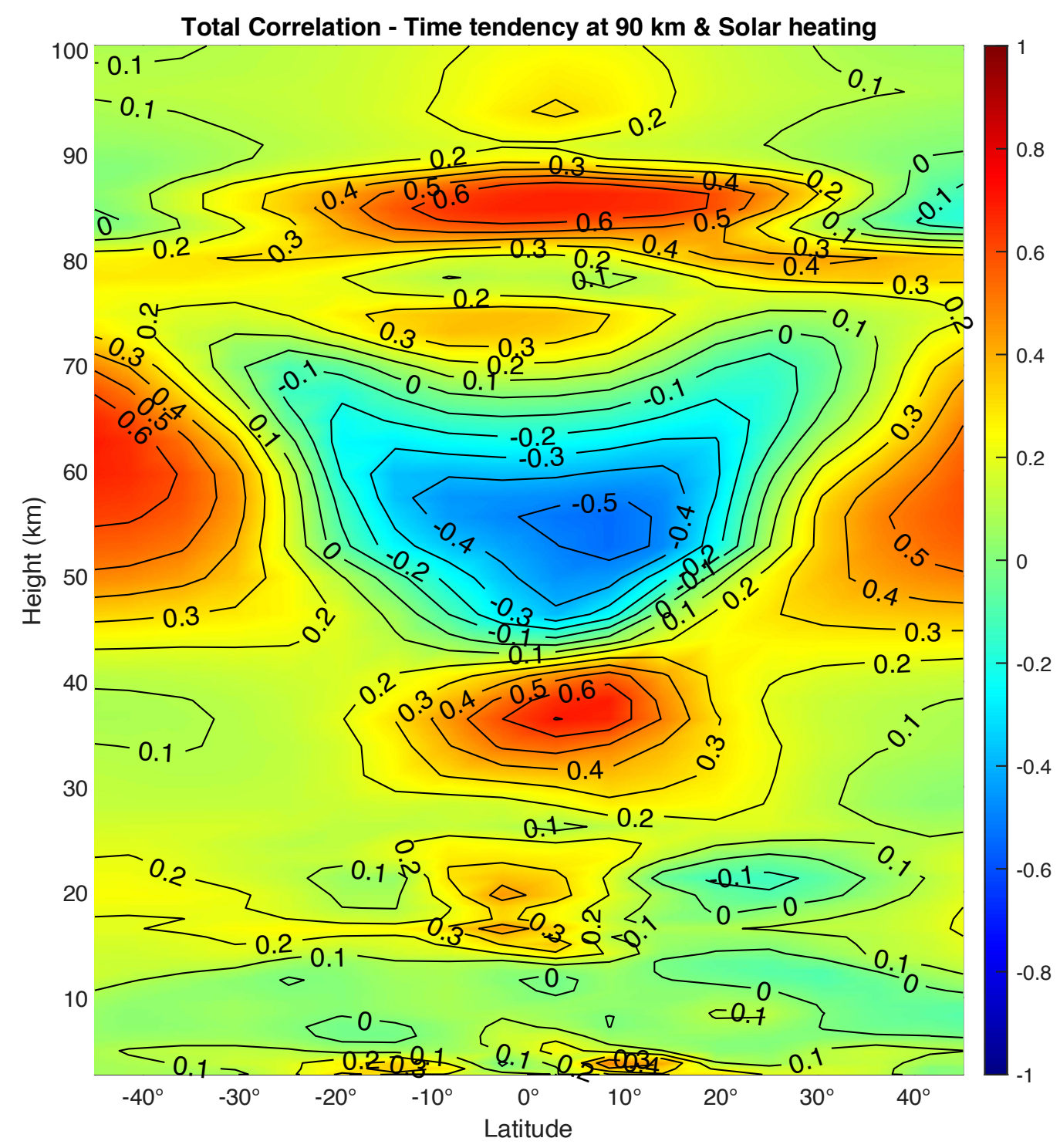

Figure C2. Correlations between solar heating at all heights with the DW1 heating rate at $90 \mathrm{~km}$ as a function of latitude and height. 


\title{
CURRICULUM VITAE
}

\author{
Lokupatabendige Ashan Shivantha Vitharana \\ Department of Physics and Astronomy \\ University of Louisville, Louisville, KY \\ lavith01@louisville.edu
}

\section{EDUCATION}

2020 Ph.D. Physics, University of Louisville, Louisville, KY.

2016 M.Sc. Physics, University of Louisville, Louisville, KY.

2012 B.Sc. Physics, University of Colombo, Colombo, Sri Lanka.

\section{RESEARCH/ TEACHING EXPERIENCE}

Instructor/Teaching Assistant, August 2014 - July 2016

- Instructor for elementary astronomy (PHYS 108)

- Instructor for fundamentals of Physics lab (PHYS 223)

- Instructor for introductory laboratories II (PHYS 296)

Graduate Research Assistant, August 2016 - August 2020

- Investigated the short-term variability (on the order of 4 days) of atmospheric tides from about the troposphere to the ionospheric $\mathrm{E}$ region dynamo region $(20-110 \mathrm{~km})$. Emphasis is on the migrating diurnal tide DW1 (westward propagating wavenumber 1$)$.

- Developed hierarchy of statistical models to forecast tidal weather.

- Studied physical mechanisms of DW1 tidal weather. 


\section{PUBLICATIONS AND PRESENTATIONS}

- Vitharana, A., Zhu, X., Du, J., Oberheide, J., \& Ward, W. E. (2019). "Statistical modeling of tidal weather in the mesosphere and lower thermosphere". Journal of Geophysical Research: Atmospheres, 124, 9011 - 9027. https://doi.org/10.1029/2019JD030573

- Vitharana, A., Zhu, X., Du, J., Oberheide, J., \& Ward, W. E. "Possible Physical Mechanisms for Quasi 23-day Oscillation in DW1 Tidal Variability in the MLT Region". American Geophysical Union (AGU) Fall Meeting 2019 (Poster).

- Vitharana, A., Du, J., Zhu, X., Oberheide, J., \& Ward, W. E. "Statistical Modeling of DE3 Tidal Weather in the MLT Region". Coupling, Energetics and Dynamics of Atmospheric Regions (CEDAR) conference, June 2019 (Poster).

- Vitharana, A., Du, J., Zhu, X., Oberheide, J., \& Ward, W. E. "Statistical Modeling of DW1 Tidal Variability in the MLT Region". American Geophysical Union (AGU) Fall Meeting 2018 (Poster).

- Vitharana, A., Du, J., Oberheide, J., \& Ward, W. E. "Characteristics of Short-term Tidal Variability of DW1 from daily to solar cycle time scales in eCMAM30 and SABER". Coupling, Energetics and Dynamics of Atmospheric Regions (CEDAR) conference, June 2018 (Poster).

- Vitharana, A., Du, J., Oberheide, J., \& Ward, W. E. "Study of Short-term Migrating (DW1) Tidal Variability in eCMAM30 and SABER Using Information Theory". Coupling, Energetics and Dynamics of Atmospheric Regions (CEDAR) conference, June 2017 (Poster).

- Vitharana, A., Du, J., Oberheide, J., \& Ward, W. E. "Statistical characteristics of short-term variability of diurnal tides in eCMAM30". Coupling, Energetics and Dynamics of Atmospheric Regions (CEDAR) conference, June 2016 (Poster).

- Vitharana, A., Du, J., Oberheide, J., \& Ward, W. E. "A comparison of statistical properties of diurnal tides between eCMAM30 and SABER". Coupling, Energetics and Dynamics of Atmospheric Regions (CEDAR) conference, June 2015 (Poster). 